\title{
WestVirginiaUniversity
}

THE RESEARCH REPOSITORY @ WVU

Graduate Theses, Dissertations, and Problem Reports

2007

\section{Growth of diesel exhaust particulate matter in a ventilated mine tunnel}

Glen A. Wilt

West Virginia University

Follow this and additional works at: https://researchrepository.wvu.edu/etd

\section{Recommended Citation}

Wilt, Glen A., "Growth of diesel exhaust particulate matter in a ventilated mine tunnel" (2007). Graduate Theses, Dissertations, and Problem Reports. 2774.

https://researchrepository.wvu.edu/etd/2774

This Dissertation is protected by copyright and/or related rights. It has been brought to you by the The Research Repository @ WVU with permission from the rights-holder(s). You are free to use this Dissertation in any way that is permitted by the copyright and related rights legislation that applies to your use. For other uses you must obtain permission from the rights-holder(s) directly, unless additional rights are indicated by a Creative Commons license in the record and/ or on the work itself. This Dissertation has been accepted for inclusion in WVU Graduate Theses, Dissertations, and Problem Reports collection by an authorized administrator of The Research Repository @ WVU.

For more information, please contact researchrepository@mail.wvu.edu. 


\title{
Growth of Diesel Exhaust Particulate Matter in a Ventilated
}

\section{Mine Tunnel}

\author{
Glen A. Wilt \\ Dissertation submitted to the \\ College of Engineering and Mineral Resources \\ At West Virginia University \\ In partial fulfillment of the requirements
}

For the degree of

\author{
Doctor of Philosophy \\ In \\ Aerospace Engineering \\ Mridul Gautam, Ph.D., Chair \\ Nigel N. Clark, Ph.D. \\ Scott W. Wayne, Ph.D. \\ Mohan Krishnamurthy, Ph.D. \\ Benjamin C. Shade, Ph.D. \\ Aleksandar D. Bugarski, Ph.D.
}

Department of Mechanical and Aerospace Engineering

Morgantown, West Virginia

2007 


\section{Abstract \\ Growth of Diesel Exhaust Particulate Matter in a Ventilated Mine Tunnel}

\section{Glen A. Wilt}

The objective of this work was to study the formation and evolutionary characteristics of aerosols, originating from the exhaust plume of a diesel engine operating in a ventilated mine tunnel. To extract these characteristics, precise cross-sectional scalar exhaust maps of $\mathrm{CO}_{2}$, temperature, and aerosol concentration were generated and used to track the time averaged axial development of the plume. These maps were then used to position aerosol size distribution samples within critical regions of the plume. This approach was found to provide an efficient and thorough record of aerosol formation and evolution, as a result of naturally dispersed diesel exhaust in mine environments.

A diesel exhaust plume is made up of a complex spatial and temporally dependent array of various exhaust constituents. The state (i.e. temperature and partial pressures) of these constituents, as they travel through space, will depend on the nature of the plume and its environment. Laboratory studies usually simulate plume processes through sudden fully mixed systems. However, this approach compromises the complex path dependent processes present within natural plumes. This discrepancy can significantly affect the trends reported for the generation and transformation of diesel exhaust aerosols, which are dependent to a large extent on fuel sulfur level, temperature, species' partial pressures, residence time, and dilution ratio. Some studies have been performed which preserve the natural evolution of the plume (i.e. vehicle chasing), but only demonstrate crude spatial development through the collection of relatively coarse and imprecisely positioned samples. As such, vehicle chasing studies result in relatively primitive descriptions of the formation and evolution of diesel exhaust aerosols. Consequently the following study was designed to efficiently extract detailed relationships that exist between the aerosols and other variables of a naturally occurring exhaust plume.

During this study, a digital three-axis probe placement device was developed and used to position time averaged exhaust samples precisely throughout the plume. Mapping software was also created and interfaced with the device, allowing a continued awareness of the relative probe positions with respect to the emerging plume. Scalar exhaust maps were extracted through the use of a NDIR $\mathrm{CO}_{2}$ analyzer, K-type thermocouple, and a handheld TSI CPC3007 particle counter. These maps were used to strategically position aerosol size distribution samples measured by a TSI Scanning Mobility Particle Sizer (SMPS). This approach was employed to save time without incurring any losses in the quality of trends found from aerosol size distribution samples.

The results of this study reveal the intricate 3-dimensional paths traveled by developing aerosols under natural mixing. These paths are marked by continually changing exhaust states known to affect aerosol evolution. As such, spatial trends observed in aerosol data were found highly diverse over distances spanning as little as a few inches. These trends showed growth in the nuclei mode at distances as far as 20 feet from the exhaust source. Beyond 20 feet, the nuclei mode experienced considerable losses nearing 1 order of magnitude at a distance of $\sim 200$ feet from the exhaust source. In spite of this loss, the accumulation mode was found virtually unaffected throughout the entire 200 foot test region of the tunnel. 
To:

Mom and Dad 


\section{Acknowledgements}

- Mridul Gautam, Ph.D.

- Aleksandar Bugarski, Ph.D. (and all other NIOSH researchers and employees of LLL)

- Nigel Clark, Ph.D.

- $\quad$ Scott Wayne, Ph.D.

- Mohan Krishnamurthy, Ph.D.

- Benjamin Shade, Ph.D.

- Leo Marbun, Aaron Barnett, Dan Carder, and Cliff Judy 


\section{Table of Contents}

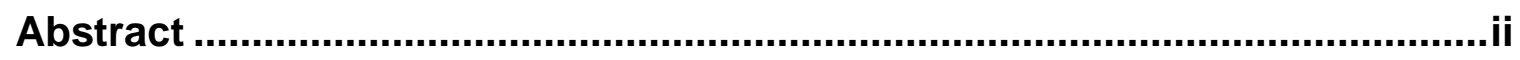

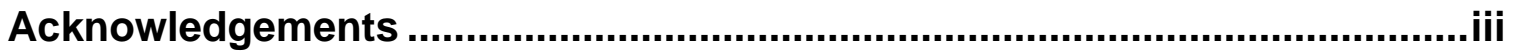

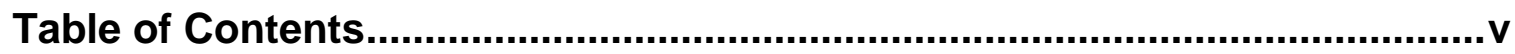

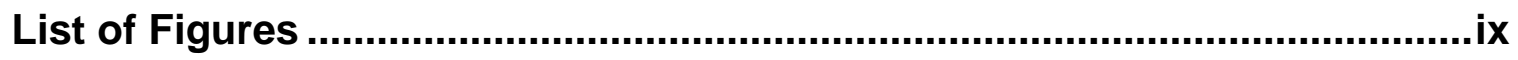

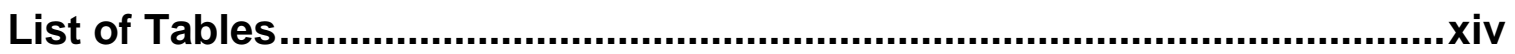

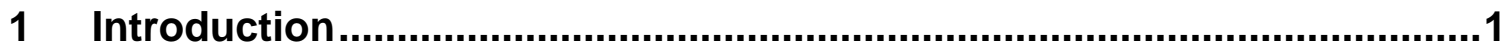

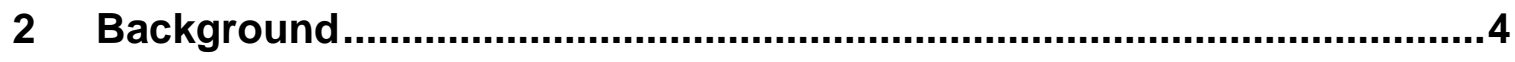

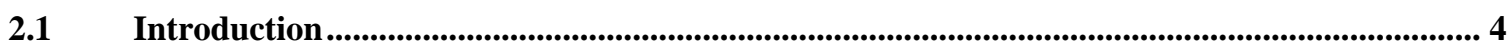

$2.2 \quad$ Aerosols / Particulate Matter (PM) ....................................................................................................... 5

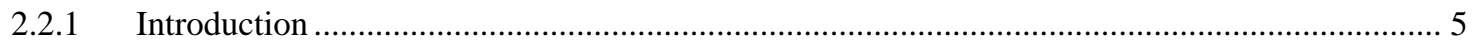

2.2.2 Characteristics of Diesel Exhaust Aerosol Size Distributions ..................................................... 5

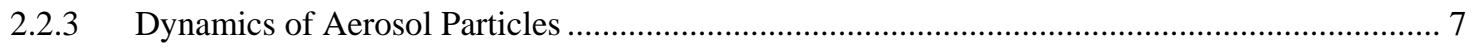

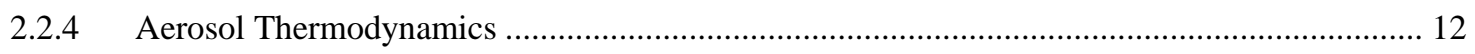

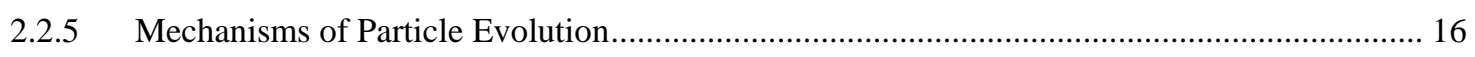

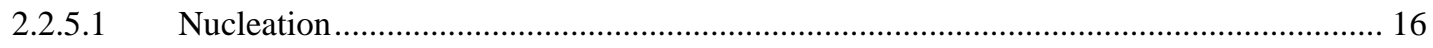

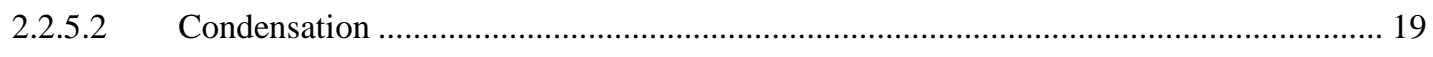

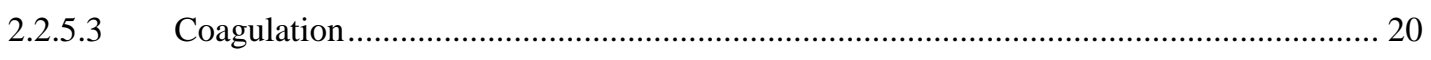

$2.3 \quad$ Data Visualization / Interpolation................................................................................................. 22 


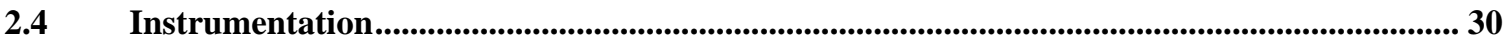

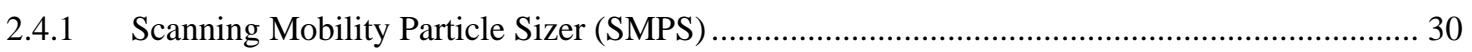

2.4.2 Model 3007 Condensation Particle Counter............................................................................... 30

$2.5 \quad$ West Virginia University Wind Tunnel Study.......................................................................... 30

3 Experimental Layout and Procedures .................................................32

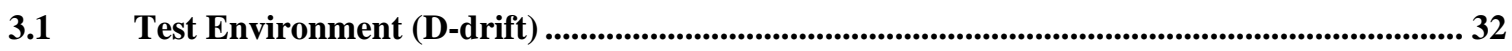

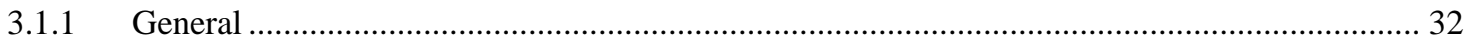

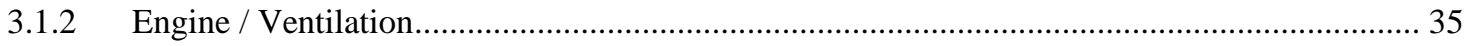

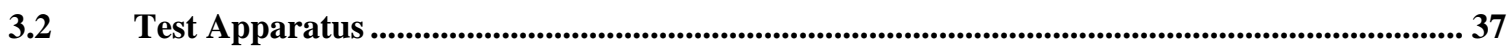

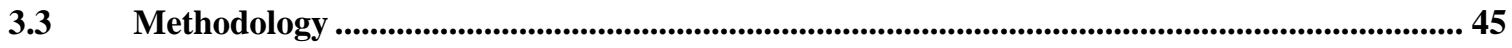

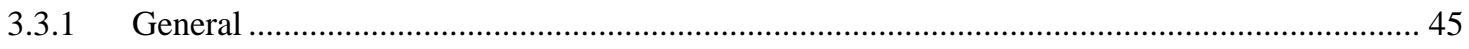

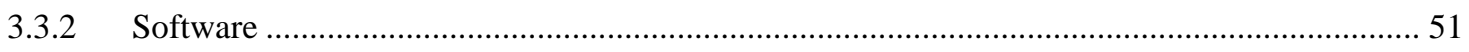

3.4 Special Mapping Concerns ............................................................................................................. 54

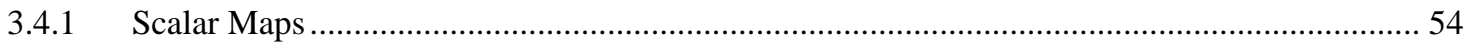

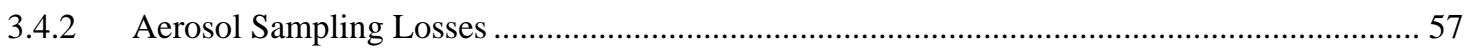

$3.5 \quad$ Plume Test Schedule ….................................................................................................................. 58

3.6 Dimensional Analysis ................................................................................................................ 59

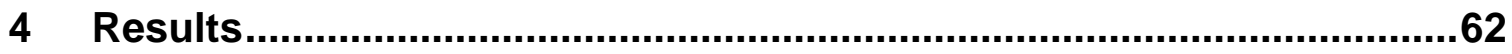

4.1 Background and Raw Exhaust Data .............................................................................................. 62

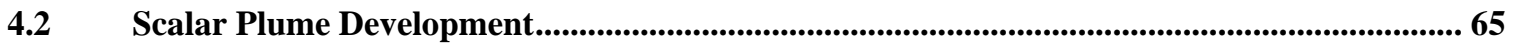

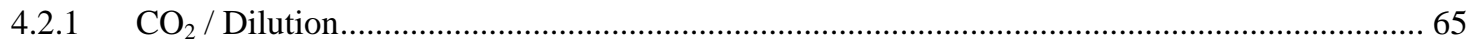

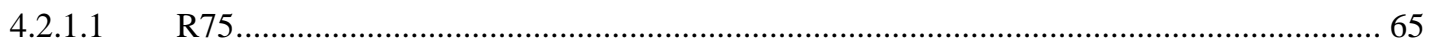




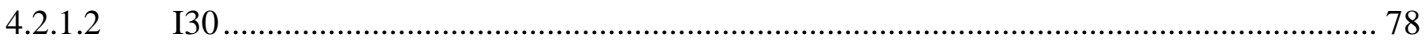

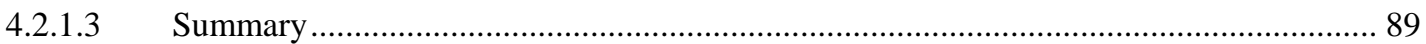

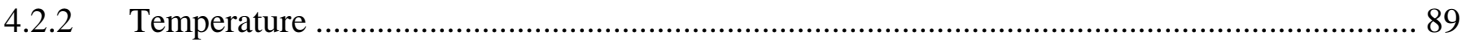

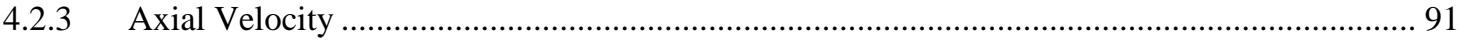

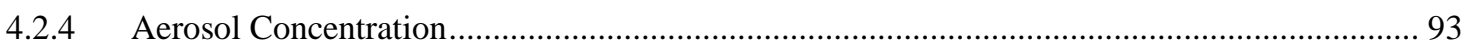

4.2.5 Axial Development and Dilution throughout Space................................................................. 94

4.2.6 Combined Development of $\mathrm{CO}_{2}$, Temperature, and Aerosol Concentration............................. 99

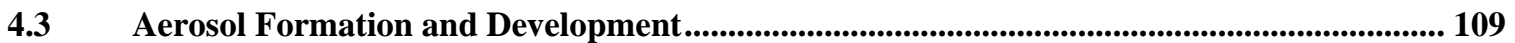

4.3.1 Scaling Aerosol Formation through Dilution ............................................................................. 109

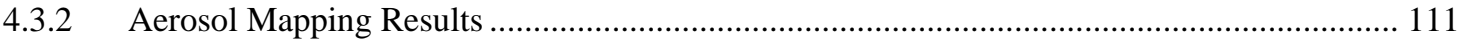

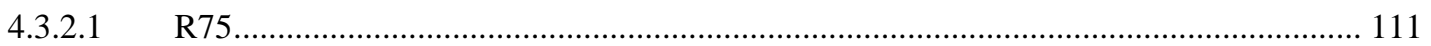

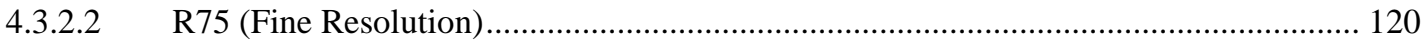

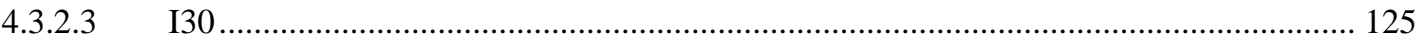

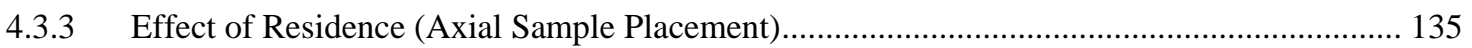

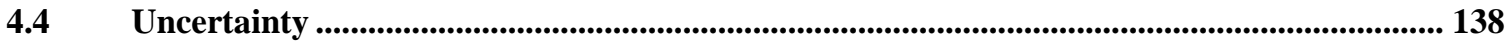

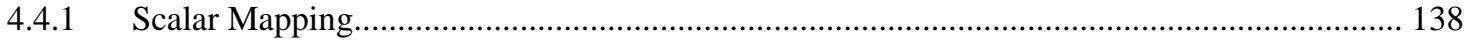

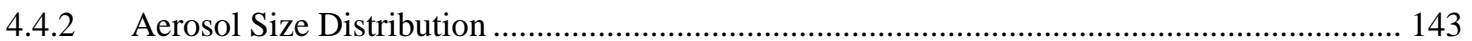

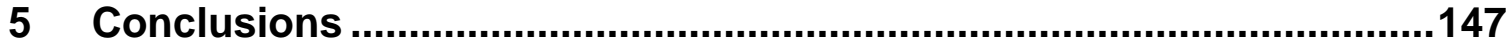

6 Future Recommendations.................................................................

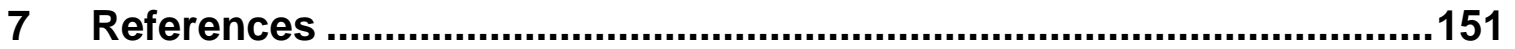

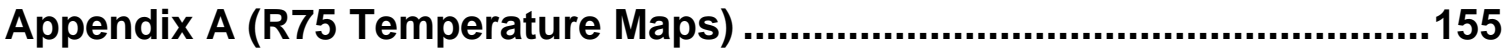

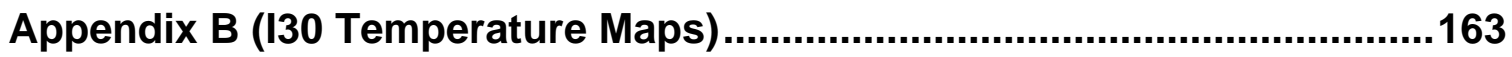


Appendix C (Mapping Code Sample) ….................................................169

Approval of the Examining Committee .................. Error! Bookmark not defined. 


\section{List of Figures}

Figure 1 Qualitative Description of the Nucleation Rate Equation [7].................................................... 18

Figure 2 Preliminary Linear Interpolations for a Square Element ........................................................... 24

Figure 3 Example Internal Interpolation for Square Element .................................................................... 25

Figure 4 Rectangular Shaped Intensity to be Mapped Through Lines L1 and L2 .............................. 28

Figure 5 Distorted Mapping Caused by Dissimilar Dimensions...................................................................... 29

Figure 6 D-drift General Arrangement ........................................................................................................ 33

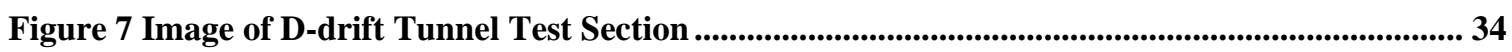

Figure 8 Frontal SMPS Shield Showing Various Probe Arrangements .................................................... 38

Figure 9 Test Apparatus Horizontal Drive Assembly .............................................................................. 40

Figure 10 Vertical Tower Assembly.................................................................................................................. 42

Figure 11 Inner Vertical Gear Assembly............................................................................................................ 43

Figure 12 Fully Assembled Test Rig ............................................................................................................... 44

Figure 13 General Axial Placement of Cross-sectional Maps (Contour levels are not drawn to the same scale for each station and are given only to suggest the geometry of the plume.) ...... 46

Figure 14 Example $\mathrm{CO}_{2}$ map (Color scale refers to ppm)............................................................................ 47

Figure 15 Axial Station Layout (Top View) ................................................................................................. 50

Figure 16 10ft Mapping Software Display Example....................................................................................... 52

Figure 17 Example 4-Dimensional Exhaust Map of Figure 14 ......................................................................... 54

Figure 18 Qualitative Example of the Effect of Regular Plume Motion on Averaging.......................... 55

Figure 19 Illustrative Example of Coupled Map Dependencies. ................................................................. 56

Figure 20 Raw Exhaust $\mathrm{CO}_{2}$ Data Taken Under R75 and I30 Operation............................................... 63

Figure 21 Raw Exhaust Temperature Data Taken Under R75 and I30 Operation. (Thermocouple

located 2 feet ahead of exhaust outlet) ........................................................................................ 63

Figure $22 \mathrm{CO}_{2}$ Mapping Results for an Axial Placement of 11in (Taken on Day 7)............................... 66

Figure $23 \mathrm{CO}_{2}$ Mapping Results for an Axial Placement of 6ft (Taken on Day 5). ............................... 67

Figure $24 \mathrm{CO}_{2}$ Mapping Results for an Axial Placement of 6ft (Taken on Day 6). ............................... 68 
Figure $25 \mathrm{CO}_{2}$ Mapping Results for an Axial Placement of 10ft (Taken on Day 7).

Figure $26 \mathrm{CO}_{2}$ Mapping Results for an Axial Placement of 20ft (Taken on Day 5). ............................. 71

Figure $27 \mathrm{CO}_{2}$ Mapping Results for an Axial Placement of 20ft (Taken on Day 6). ........................... 72

Figure $28 \mathrm{CO}_{2}$ Mapping Results for an Axial Placement of 50ft (Taken on Day 7). .......................... 73

Figure 29 Hanging Obstruction Found Ahead of the 50ft Axial Station ............................................ 74

Figure $30 \mathrm{CO}_{2}$ Mapping Results for an Axial Placement of 125ft (Taken on Day 5). .......................... 76

Figure $31 \mathrm{CO}_{2}$ Mapping Results for an Axial Placement of 125ft (Taken on Day 6). ......................... 77

Figure $32 \mathrm{CO}_{2}$ Mapping Results for an Axial Placement of 11in (Taken on Day 10)........................ 79

Figure $33 \mathrm{CO}_{2}$ Mapping Results for an Axial Placement of 6ft (Taken on Day 8). ............................ 80

Figure $34 \mathrm{CO}_{2}$ Mapping Results for an Axial Placement of 10ft (Taken on Day 8). ........................... 81

Figure 35 Example single point data used to obtain an average for both engine settings................... 82

Figure $36 \mathrm{CO}_{2}$ Mapping Results for an Axial Placement of 25ft (Taken on Day 10). ....................... 83

Figure $37 \mathrm{CO}_{2}$ Mapping Results for an Axial Placement of 100ft (Taken on Day 10). ....................... 84

Figure $38 \mathrm{CO}_{2}$ Mapping Results for an Axial Placement of 125ft (Taken on Day 9). .......................... 85

Figure $39 \mathrm{CO}_{2}$ Mapping Results for an Axial Placement of 150ft (Taken on Day 9). ........................ 86

Figure $40 \mathrm{CO}_{2}$ Mapping Results for an Axial Placement of 150ft (Taken on Day 9) With Suspected

“Bad” Points Taken Out. (Color Scale Refers to CO2 concentration (ppm))..................... 87

Figure 41 Effect of Door Opening on the Collection of a Single Point Average................................... 88

Figure 42 Temperature Mapping Results for an Axial Placement of 10ft (Taken on Day 7)............. 90

Figure 43 Temperature Mapping Results for an Axial Placement of 150ft (Taken on Day 9)............ 91

Figure 44 Axial Velocity Mapping Results for an Axial Placement of 10ft (Taken on Day 8)............. 92

Figure 45 Aerosol Concentration Mapping Results for an Axial Placement of 10ft (Taken on Day 7).

Figure 46 Example Vertical Axial Cut through Tunnel Center (Coordinate pairs represent axial and vertical distance in inches. The color scale represents CO2 concentration (ppm)) ............ 95

Figure 47 Example Constant Dilution (DR=27) Shell Taken from Test Days 5 through 7. (the color scale refers to axial tunnel distance in inches) 96 
Figure 48 Example of a Constant Dilution Surface that Splits into a Stratified Layer at the Tunnel Ceiling. (Generated from data taken on test days 5 through 7. Color scale refers to axial

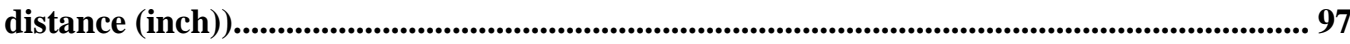

Figure 49 Illustration of a Constant Dilution Surface Involving a Transition into Stratification....... 98 Figure 50 Simultaneous $\mathrm{CO}_{2}$ and Temperature Comparison Taken on Day 7 at a 10ft Axial Placement. (Elevation Bands Refer to $\mathrm{CO}_{2}$ contours while Color Refers to Temperature)

Figure 51 Simultaneous $\mathrm{CO}_{2}$ and Temperature Comparison Taken on Day 5 at a 125ft Axial

Placement. (Elevation Bands Refer to $\mathrm{CO}_{2}$ contours while Color Refers to Temperature)

Figure 52 The Simultaneous Comparison of $\mathrm{CO}_{2}$ and Temperature Expansion Patterns Found in

Figure 50. (Black and Red Bands Represent $\mathrm{CO}_{2}$ and Temperature $10 \%$ Contours

Respectively)

Figure 53 Temperature Variations for the $\mathrm{CO}_{2}$ Contours Taken on Day 7 at an Axial Placement of

10ft. (Color Scale refers to Temperature in C)

Figure 54 Resulting Scalar Coordinate Grid Obtained from a Uniform Spatial Grid. (10ft axial placement taken on day 7)

Figure 55 Aerosol Concentration Dependence on $\mathrm{CO}_{2}$ and Temperature at an Axial Placement of 10ft Taken on Day 7. (Color scale is un-calibrated and represents trends in PM concentration $\left(\# / \mathrm{cm}^{3}\right)$ only)........................................................................................................ 105

Figure 56 Example Constant Dilution Surface Painted by Temperature............................................... 107

Figure 57 Absolute Aerosol Size Distribution Results at Specified Dilutions........................................ 109

Figure 58 Scaled Aerosol Size Distribution Results at Specified Dilutions................................................ 110

Figure 59 6ft Aerosol Size Distribution Measurement Locations for Figure 60 ..................................... 111

Figure 60 6ft Aerosol Size Distribution Results for Days 5 and 6 ......................................................... 112

Figure $6110 \mathrm{ft}$ Aerosol Size Distribution Measurement Locations for Figure 62 ................................ 113

Figure 62 10ft Aerosol Size Distribution Results for Day 7. ....................................................................... 114

Figure 63 20ft Aerosol Size Distribution Measurement Locations for Figure 64 .................................. 115 
Figure 64 20ft Aerosol Size Distribution Results ......................................................................................... 115

Figure 65 50ft Aerosol Size Distribution Measurement Locations for Figure 66 ................................ 117

Figure 66 50ft Aerosol Size Distribution Results ........................................................................................ 117

Figure 67 125ft Aerosol Size Distribution Measurement Locations for Figure 68 ................................ 119

Figure 68 125ft Aerosol Size Distribution Results ......................................................................................... 119

Figure 69 1ft DR Channel Comparison and Selected Blend........................................................................ 120

Figure 70 1ft Aerosol Size Distribution Measurement Locations for Figure 71 ................................... 121

Figure 71 1ft Aerosol Size Distribution Results ....................................................................................... 122

Figure 72 3ft Aerosol Size Distribution Measurement Locations for Figure 73 ................................... 123

Figure 73 3ft Aerosol Size Distribution Results ......................................................................................... 123

Figure 74 10ft Aerosol Size Distribution Measurement Locations for Figure 75 ................................. 124

Figure 75 10ft Aerosol Size Distribution Results. ........................................................................................... 125

Figure 76 1ft Aerosol Size Distribution Measurement Locations for Figure 77 ................................... 126

Figure 77 1ft Aerosol Size Distribution Results. .............................................................................................. 126

Figure 78 6ft Aerosol Size Distribution Measurement Locations for Figure 79 .................................... 127

Figure 79 6ft Aerosol Size Distribution Results .......................................................................................... 128

Figure $8010 \mathrm{ft}$ Aerosol Size Distribution Measurement Locations for Figure 81 ................................ 129

Figure 81 10ft Aerosol Size Distribution Results. ................................................................................. 129

Figure 82 25ft Aerosol Size Distribution Measurement Locations for Figure 83 ................................. 130

Figure 83 25ft Aerosol Size Distribution Results ............................................................................................ 131

Figure 84 100ft Aerosol Size Distribution Measurement Locations for Figure 85 ............................... 132

Figure 85 100ft Aerosol Size Distribution Results ........................................................................................ 132

Figure 86 125ft Aerosol Size Distribution Measurement Locations for Figure 87 .................................. 133

Figure 87 125ft Aerosol Size Distribution Results ............................................................................................ 134

Figure 88 Effect Residence on Aerosol Size Distribution (R75 operation) ........................................... 136

Figure 89 Effect of Residence on Aerosol Formation (DR=35) ............................................................... 137

Figure 90 Schematic of Study to Determine Foreign Object Interference on Mapping Results. ...... 139

Figure $91 \mathrm{CO}_{2}$ Mapping Results for Various Blockage Arrangements. ................................................ 140 
Figure 92 Convergence of $\mathrm{CO}_{2}$ Map Along an Arbitrary Line. (Taken on day 7 at 10 feet)

Figure 93 Convergence of $\mathrm{CO}_{2}$ Map Along an Arbitrary Line. (Taken on day 6 at 20 feet) 142

Figure 94 Aerosol Size Distribution Results with Error Bars (day 5 6ft) 144

Figure 95 Aerosol Size Distribution Results with Error Bars (day 5 125ft). 145

Figure 96 Aerosol Size Distribution Results with Error Bars (day 10 25ft). 146

Figure 97 Temperature Mapping Results for an Axial Placement of 11 inches (Taken on Day 7 )... 155

Figure 98 Temperature Mapping Results for an Axial Placement of 6 ft (Taken on Day 5)............. 156

Figure 99 Temperature Mapping Results for an Axial Placement of $6 \mathrm{ft}$ (Taken on Day 6)............ 157

Figure 100 Temperature Mapping Results for an Axial Placement of $20 \mathrm{ft}$ (Taken on Day 5)........ 158

Figure 101 Temperature Mapping Results for an Axial Placement of $20 \mathrm{ft}$ (Taken on Day 6)........ 159

Figure 102 Temperature Mapping Results for an Axial Placement of $50 \mathrm{ft}$ (Taken on Day 7)........ 160

Figure 103 Temperature Mapping Results for an Axial Placement of $125 \mathrm{ft}$ (Taken on Day 5)...... 161

Figure 104 Temperature Mapping Results for an Axial Placement of $125 \mathrm{ft}$ (Taken on Day 6)....... 162

Figure 105 Temperature Mapping Results for an Axial Placement of 11 inches (Taken on Day 10).

Figure 106 Temperature Mapping Results for an Axial Placement of 6 ft (Taken on Day 8).......... 164

Figure 107 Temperature Mapping Results for an Axial Placement of $10 \mathrm{ft}$ (Taken on Day 8)......... 165

Figure 108 Temperature Mapping Results for an Axial Placement of $25 \mathrm{ft}$ (Taken on Day 10)...... 166

Figure 109 Temperature Mapping Results for an Axial Placement of $100 \mathrm{ft}$ (Taken on Day 10).... 167

Figure 110 Temperature Mapping Results for an Axial Placement of $125 \mathrm{ft}$ (Taken on Day 9)...... 168 


\section{List of Tables}

Table 1 Aerosol Size Range Definitions ................................................................................................................. 7

Table 2 Summary of Chemical Potentials [7]..................................................................................................... 13

Table 3 Key Characteristicss of Isuzu C240 [13] .......................................................................................... 35

Table 4 Relevant C240 Modes of Operation Characteristics........................................................................... 36

Table 5 Plume Test Schedule ............................................................................................................... 58

Table 6 Summary of Dimensional Characteristics of Plume Study ......................................................... 59

Table 7 Summary of Recorded Background and Raw Exhaust Measurements...................................... 64 


\section{Introduction}

The objective of this work was to study the formation and evolutionary trends of aerosols, originating from a diesel exhaust engine within a ventilated mine tunnel. In addition, an efficient procedure for the collection of comprehensive aerosol size distribution trends was developed. These trends were also compared to the evolution of

other exhaust quantities to develop an understanding of the natural connections existing between each variable of the plume.

This study differs from work performed in the past in that the exhaust flow was not assumed or encouraged to be mixed for any part of the tunnel. Instead, precise crosssectional scalar exhaust maps were generated and used to track the time averaged axial development of the plume. These maps were then used to position aerosol size distribution samples within critical regions of the plume. This approach was shown to provide an efficient and thorough record of diesel aerosol production in mine environments, which complements previous studies that have neglected the precise spatial evolution of the plume.

Detailed spatial exhaust plume mapping of the past has been overshadowed by the application of various test metrics including averaged dilution ratio and residence time. These metrics have been employed in order to assimilate vast quantities of emissions studies data. In laboratory confined rapid mixing environments, these metrics may provide valuable trendworthy results. However, in natural dissipative environments, they might not be as well suited in describing plume behavior. In such cases it may become important to view the plume in terms of its spatial and temporal development in addition to other test metrics used in the past.

In natural (i.e. out of lab) environments, plume development is a highly complex multidimensional process. The plume is made up of a large number of gases and particles each existing over differing concentrations and states throughout space and time. These gases and particles naturally possess unique diffusive properties. Consequently, the internal structure and composition of the plume evolves as its spatial influence grows. As such, using distinct gases as a means to describe the expansion of the exhaust as a whole may result in un-intentioned complexity in observed trends within collected data. 
In addition, natural dilution takes place over three spatial dimensions. As such, a single dilution level will define a 3-dimensional surface. It is plausible that other exhaust quantities over this surface will not remain constant. Therefore, references to dilution alone might inadvertently assimilate dissimilar data. Consequently, most studies also pair what is known as a residence time with each measured quantity. These times are typically determined based on bulk flow exhaust velocities. However, each point of the surface represents a unique path of origin spanning multiple exhaust dimensions of possible dissimilarity. Therefore, any two points of the surface that happen to share a common residence time may be the result of vastly different exhaust paths. Therefore, as in dilution metrics, residence time may also tend to group dissimilar data. In cases where the above mentioned effects cannot be ignored, it may become necessary to map the plume as a detailed spatial entity.

Spatial exhaust plume development is perhaps of most concern in enclosed working environments such as in underground mines. These environments are responsible for some of the highest occupational exhaust exposures. The National Institute for Occupational Safety and Health (NIOSH [2]) noted that miners are exposed to as much as 10 times more particulate matter (PM) from diesel exhaust than all other high risk occupations. Since a diesel exhaust plume naturally rises due to buoyancy, highly concentrated regions of exhaust influence may persist throughout time for particular applications. These regions may need to be studied in detail to determine any affect they may have on the formation and evolution of diesel exhaust aerosols.

This study explored spatial concentration variations in diesel exhaust pollutants within a typical mine tunnel (D-drift). D-drift is a part of Lake Lynn Laboratory (LLL), a research underground mine facility established by NIOSH. In addition to an understanding of natural diesel exhaust aerosol developmental processes in mine environments, tests performed here had the added benefit of establishing fully mixed conditions for the tunnel. Fully mixed conditions were to be achieved, by NIOSH researchers, for the evaluation of bulk emissions production in mine environments.

Tests performed in D-drift provided a real-world yet semi-controlled (i.e. venturi metered ventilation) confined environment which reduced the mapping complexity 
observed in outdoor and other applications. The plume was mapped using time averaged measurements of exhaust parameters. A test apparatus was designed and built to carry PM sizing instrumentation and other sensors needed to map the plume accurately and precisely throughout the tunnel. Through this apparatus, a number of single point exhaust averages were taken in an effort to produce precise scalar exhaust maps capable of directing further more time consuming measurements of PM. In this study, detailed maps were generated using a k-type thermocouple for temperature maps, a California Analytical $\mathrm{CO}_{2}$ concentration analyzer for near and far field $\mathrm{CO}_{2}$ maps (15 and 5\%), and a handheld TSI CPC3007 particle counter for aerosol concentration maps. The $\mathrm{CO}_{2}$ maps were then used to guide the placement of PM size distribution measurement equipment such as the TSI Scanning Mobility Particle Sizer (SMPS) within critical regions of the plume. With critical characteristics of the plume resolved, this approach has provided an accurate and comprehensive record of diesel aerosol size distribution in the particular mine environment that was studied. 


\section{Background}

\subsection{Introduction}

This study is an empirical attempt to understand the processes that influence the transformation of diesel exhaust aerosols in mining environments, by mapping them for a particular mine geometry. Primary emphasis was placed on the formation of nanoparticles (5 to $50 \mathrm{~nm}$ ), as they have become the subject of adverse health concerns due to their high number and lung penetrating abilities.

Underground miner's exposure to diesel exhaust PM is significantly higher than of the other high risk occupations. Schnakenberg and Bugarski [2] cited metal / nonmetal underground mine exposures to diesel exhaust PM in the range of 0.3 to $1.6 \mathrm{mg} / \mathrm{m}^{3}$ while forklift operator exposures were only in the range of 0.02 to $0.1 \mathrm{mg} / \mathrm{m}^{3}$. Due to their low contribution to mass, nanoparticle levels may be unrepresented by these numbers. Consequently, nanoparticle levels in mine environments may demonstrate even more profound differences, either favorable or unfavorable.

Nanoparticle formation is an extremely complex and sensitive process. Kittelson and Abdul-Khalek [1] noted nanoparticle concentration levels varying by more than two orders of magnitude for "normal variation in ambient dilution.” The potential for exhaust to rise and collect in certain isolated regions of a mine could produce spatial dilution variations much more significant than those observed in Kittelson and Abdul-Khalek's study. As such, even higher variations may be observed in nanoparticle levels.

To date, no work has been found that precisely maps these variations for a specific mining application and geometry, which is the objective of this study. 
The following sections provide a scientific background beneficial to the understanding of this study and its results. A few sections are also provided as background for the devices and techniques used and developed within the study.

\subsection{Aerosols / Particulate Matter (PM)}

\subsubsection{Introduction}

An aerosol is defined as the combination of suspended particles or droplets in a gaseous medium. The medium is usually treated as continuous, having a much smaller average molecular spacing than the particles themselves. Suspended particles are differentiated from the gas in which they reside through both their size and / or composition. This differentiation carries over into the physical behavior of the particle with respect to the gas. These physical distinctions are communicated in the form of heat, mass and momentum transfer between the two systems. Predictions in quantifying these transfers and descriptions of the mechanisms through which they originate are the objectives of aerosol science. The following sections describe some important aspects of this science and its application to diesel exhaust aerosols.

\subsubsection{Characteristics of Diesel Exhaust Aerosol Size Distributions}

Diesel exhaust aerosol size distributions are generally characterized by three modes. The smallest mode (nuclei mode 5 to $50 \mathrm{~nm}$ ) typically accounts for more than 90 percent of the total aerosol number while only 1 to 20 percent of the total aerosol mass [3]. These particles are generally formed by nucleation due to the super saturation of volatile components of exhaust during dilution and are typically not present until the exhaust has released from the tail pipe. The composition of these particles is thought to consist of 
metallic compounds, elemental carbon, and semi-volatile organic and sulfur compounds [4].

The next largest mode (accumulation mode 50 to $500 \mathrm{~nm}$ ) is dominated by particles formed through the coagulation of ash particles and carbonaceous products of incomplete combustion [5]. Unlike nucleated particles, the growth in number of these particles is not favored by dilution. Brown et al. [5] mentions that, due to the second order kinetic nature of coagulation, a dilution of 1000:1 would reduce the coagulation rate by a factor of 1,000,000. As such, these particles are not as readily formed or transformed outside of the exhaust pipe as they are inside the exhaust pipe.

Since particles of the accumulation mode grow in the presence of volatile material through adsorption, they act as competitors with the formation of nucleation mode particles. Therefore, a distribution with a large or small accumulation mode should be accompanied by a small or large nucleation mode, respectively. An example of this can be found in [6] which demonstrated nucleation number increases as high as 30 to 60 fold for substantial decreases in total aerosol mass. Since the majority of aerosol mass is contained in the accumulation mode, these findings suggest the inverted dependence of nucleation mode particles on the accumulation mode.

The presence of the largest particle mode (coarse mode $>1 \mu \mathrm{m}$ ) is due to material that is freed from the walls of the exhaust system during operation. This mode is typically small in number but accounts for 5 to 20 percent of total aerosol mass for modern diesel systems [3]. This mode will settle out of the exhaust early compared to the other size modes due to gravitational influences. 
Table 1 provides an overview of the size range definitions used for atmospheric particles, spanning all sizes present in diesel exhaust [3].

Table 1 Aerosol Size Range Definitions

\begin{tabular}{|c|c|c|c|c|}
\hline $\begin{array}{c}\text { Particle Group } \\
\text { Name }\end{array}$ & PM10 & Fine Particles & $\begin{array}{c}\text { Ultrafine } \\
\text { Particles }\end{array}$ & Nanoparticles \\
\hline Size Range & $<10 \mu \mathrm{m}$ & $<2.5 \mu \mathrm{m}$ & $<100 \mathrm{~nm}$ & $<50 \mathrm{~nm}$ \\
\hline
\end{tabular}

\subsubsection{Dynamics of Aerosol Particles}

Many scenarios can result in momentum loss or gain to a particle. These scenarios can be divided into two groups of interactions (External or Fluid). External interactions are described by momentum transfers which are not the result of fluid contact with the particle. These include transfers produced by electrical charge and gravity. Fluid interactions are the result of the particle's contact with the fluid. These include drag, diffusion, and phoretic effects. This section provides an overview of the different ways a particle can gain or lose momentum and the resulting impact on the particle's motion.

\section{Fluid Interactions}

The way a particle interacts with a gas is greatly affected by the particle's size in relation to the gase's mean free path $(\lambda)$. Particles much larger than $(\lambda)$ will experience average fluid properties and behaviors such as pressure and drag. On the contrary, particles approaching sizes similar to $\lambda$ will behave more like gas particles themselves and will not be directly influenced by such average based quantities.

Drag on a particle always acts to reduce the relative velocity between the particle and the surrounding gas. For large particles this can be expressed as: 


$$
F_{\text {Drag }}=0.5 C_{D} A_{P} \rho U_{\infty}^{2}
$$

Equation 1

Where, $F_{\text {Drag }}$ is the force on the particle due to drag, $A_{P}$ is the particle's frontal area, $\rho$ is the density of the gas and $U_{\infty}$ is the relative velocity of the particle with respect to the gas. $C_{D}$ is the particle's drag coefficient, typically calculated as a function of Reynolds number (Re). An approximation of $C_{D}$ for Reynolds numbers less than 0.1 is given by Stokes' law as

$$
C_{D}=24 / \mathrm{Re}
$$

Equation 2

Corrections to this equation can be used to predict effective drag on smaller particles which approach sizes similar to the mean free path. The following is known as the slip correction factor $\left(C_{C}\right)$ whose particular constants were determined experimentally by Allen and Raabe [15]

$$
C_{C}=1+\frac{2 \lambda}{D_{P}}\left[1.257+0.4 \exp \left(-\frac{1.1 D_{P}}{2 \lambda}\right)\right]
$$

The drag on a spherical particle of any diameter $D_{P}$ can then be calculated, as done by Seinfeld in [7], as:

$$
F_{\text {Drag }}=\frac{3 \pi \mu U_{\infty} D_{P}}{C_{C}}
$$

Where, $\mu$ is the gas dynamic viscosity.

Since $C_{C}$ must be greater than one, the resulting drag becomes smaller than predicted by Equation 1. In addition, as particle diameter lessens, $C_{C}$ increases and effective drag on a particle decreases. This can be understood as the tendency of smaller particles to slip through the gaps of the parent gas. 
It is important to mention that the application of Equation 4 for any particle assumes average drag and will hence be useful only in determining average motion. For large particles this assumption produces small differences between average and instantaneous velocities. For small particles however, variations from average drag could be strong enough to produce highly irregular disturbances in the particle’s instantaneous motion. These disturbances arise from the individual bombardment of gas molecules with the particle and are termed Brownian motion.

Consider a particle experiencing zero drag. Under zero drag, the particle will not accumulate any total travel distance. It will however, travel an integrated absolute distance due its Brownian motion. Through some mathematical manipulation Seinfeld [7] provides the average square distance $\left(<d^{2}>\right)$ traveled by a particle in any direction for a period of time $(t)$ as

$$
<d^{2}>=\frac{2 k T C_{C} t}{3 \pi \mu D_{P}}
$$

Where, $\mathrm{k}$ is the Boltzmann constant and $\mathrm{T}$ is the temperature of the surrounding gas.

An important consequence of Brownian motion is the tendency of particles to travel from high to low concentration regions. This process is termed particle diffusion. Brownian diffusivity $(D)$ is defined for a concentration field $N(X, t)$ as

$$
\frac{\partial N(X, t)}{\partial t}=D \nabla^{2} N(X, t)
$$

Equation 6

Where, $X$ is a coordinate vector inside the field.

By combining this with Equation 5, Seinfeld [7] results in a Brownian diffusivity of

$$
D=\frac{k T C_{C}}{3 \pi \mu D_{P}}
$$


Equations 6 and 7 are only valid in predicting particle diffusivity due to particle gradients. Gradients formed within the parent gas itself can also provide mechanisms for particle diffusion. These mechanisms are termed phoretic diffusion and include particle diffusion due to gas gradients in temperature (thermophoresis) and concentration (diffusiophoresis).

Temperature gradients, within a gas, result in unequal bombardment energy across the size of the particle. As such, the particle will experience an unbalanced force and tend to move in the direction of lower temperature. Larger particles will be more affected by this mechanism as their size results in larger imbalances. In addition, particles with lower thermal conductivities will be impacted more as the thermal energy potential is not merely adsorbed into the particle. Brock [16] has concluded a relationship for the resulting thermophoretic force $\left(F_{T h}\right)$ on a particle as

$$
F_{T h}=-\frac{9 \pi \mu_{\text {air }}^{2} R_{P}\left(k_{\text {air }}+c_{t} k_{P} K n\right) \nabla T}{\rho_{\text {air }} T\left(1+3 c_{m} K n\right)\left(k_{P}+2 k_{\text {air }}+2 c_{t} k_{P} K n\right)} \quad \text { Equation } 8
$$

Where $R_{P}$ is the particle radius, $K n$ is the Knudsen number $\left(K n=\lambda / R_{P}\right), c_{m}$ and $c_{t}$ are the isothermal slip and temperature jump coefficients respectively, and $k_{\text {air }}$ and $k_{P}$ are the thermal conductivities of the air and particle respectively.

Similar to the thermophoretic force is the force produced on a particle in a direction along gas gradients. This force can occur when there are three components to the aerosol system (particles, background gas B, and the diffusing gas A). Gas flux due to the presence of gradients produces a two way motion between the background and diffusing gas. The stronger of the two dictates an unequal force on the particles present in the 
gases. As such, the particle need not move in the direction of lower diffusion gas

concentration. The resulting particle velocity $\left(V_{\text {Diff }}\right)$ is provided by Seinfeld [7] as:

$$
V_{D i f f}=-\left(\frac{M_{A}^{0.5}}{y_{A} M_{A}^{0.5}+\left(1-y_{A}\right) M_{B}^{0.5}}\right) \frac{D_{A B}}{1-y_{A}} \nabla y_{A} \quad \text { Equation } 9
$$

Where, $M_{A}$ and $M_{B}$ are the molecular weights of the diffusing gas (A) and background gas (B), respectively. $D_{A B}$, is the diffusion coefficient of gas A with respect to gas B. $y_{A}$, is the local mole fraction of gas A.

Till this point, all particle interactions have been described by contact with the parent gas of the aerosol system. The following section describes a few external influences on particle motion.

\section{External Interactions}

Interactions with a particle not requiring fluid contact are termed external. The two primary external sources of particle interaction are given by gravity and electric charge. If gravity is the only force (other than drag) acting on a particle of diameter ( $\left.D_{P}\right)$, a force balance using Stoke's law results in a terminal settling velocity $\left(V_{\text {Term }}\right)$ of [7].

$$
V_{\text {Term }}=\frac{m_{P} C_{C} g}{3 \pi \mu D_{P}}
$$

Equation 10

Where $(g)$ is the gravitational constant and $\left(m_{P}\right)$ is the particle mass.

Similarly, a particle with an electric charge $(q)$ inside an electric field of strength ( $E$ ) results in a terminal settling velocity $\left(V_{\text {Term }}\right)$ of [7].

$$
V_{\text {Term }}=\frac{q C_{C} E}{3 \pi \mu D_{P}}
$$




\subsubsection{Aerosol Thermodynamics}

The following section provides an overview of the thermodynamic principles important to the study of aerosols. This section is aimed to provide equilibrium conditions for aerosol systems consisting of multiple species. A more complete description can be found in Atmospheric Chemistry and Physics [7].

The first law of thermodynamics (for a closed system) states that the differential internal energy $(d U)$ follows.

$$
d U=d Q+d W
$$

Equation 12

Where $d Q$ and $d W$ are the differential amount of heat and work applied to the system respectively.

If the system is reversible and contains ( $k$ ) species, Seinfeld [7] concludes a differential internal energy written as.

$$
d U=T d S-p d V+\sum_{i=1}^{k} \mu_{i} d n_{i}
$$

Where, $S$ and $V$ are the entropy and volume of the system respectively. $\left(n_{i}\right)$ and $\left(\mu_{i}\right)$ are the number of moles and the chemical potential of species (i) respectively. $\left(\mu_{i}\right)$ is given by

$$
\mu_{i}=\left(\frac{\partial U}{\partial n_{i}}\right)_{S, V, n_{j}}
$$

This quantity represents the system internal energy derivative with respect to species (i) obtained while entropy, volume and all other specie concentrations remain unchanged.

To resolve the difficulty in using entropy and volume differentials, the Gibbs free energy $(G)$ is introduced. 


$$
G=U+p V-T S
$$

Equation 15

Seinfeld [7] uses this definition along with the expression for ( $d U$ ) found in Equation 13 to come up with the following expression for the Gibbs free energy differential.

$$
d G=-S d T+V d p+\sum_{i=1}^{k} \mu_{i} d n_{i}
$$

Equation 16

Through further analysis of a system with constant temperature and pressure Seinfeld [7] results in a general expression for the Gibbs free energy.

$$
G=\sum_{i=1}^{k} \mu_{i} n_{i}
$$

Equation 17

The differential of $(G)$ can then be taken and combined with Equation 16 to produce the Gibbs-Duhem equation.

$$
-S d T+V d p=\sum_{i=1}^{k} n_{i} d \mu_{i}
$$

Equation 18

This equation is a statement that the chemical potentials are functions of temperature and pressure. The following table provides expressions for chemical potentials for a multitude of states.

Table 2 Summary of Chemical Potentials [7]

\begin{tabular}{|l|l|l|}
\hline Aerosol State & $\begin{array}{l}\text { Chemical Potential }\left(\mu, \mu_{i}\right) \\
\text { Relationship }\end{array}$ & Term Description \\
\hline Ideal Gas & $\mu(T, p)=\mu^{o}(T, 1 a t m)+R T \ln p$ & $\mu^{o}$ is calculated at $1 \mathrm{~atm}$. \\
\hline Ideal Solution & $\mu_{i}(T, p)=\mu_{i}^{*}(T, p)+R T \ln x_{i}$ & $\begin{array}{l}x_{i} \text { is the mole fraction of } \\
\text { species i. } \mu_{i}^{*} \text { is the chemical } \\
\text { potential for } x_{i}=1 .\end{array}$ \\
\hline Non-Ideal Solution & $\mu_{i}(T, p)=\mu_{i}^{*}(T, p)+R T \ln \alpha_{i}$ & $\alpha_{i}$ is the activity of species i. \\
\hline Solid Compound & $\mu_{i}(T, p)=\mu_{i}^{*}(T, p)$ & \\
\hline
\end{tabular}


The following describes how the chemical potentials of Table 2 can be used to determine chemical equilibrium for a system of constant temperature and pressure.

Consider the general reaction

$$
\sum a_{i} A_{i} \Leftrightarrow \sum b_{i} B_{i}
$$

Equation 19

Where, $a_{i}$ and $b_{i}$ are the stoichiometric coefficients of the $i^{\text {th }}$ reactant $\left(A_{i}\right)$ and reagent $\left(B_{i}\right)$, respectively. This equation can be written as

$$
\sum d_{i} D_{i}=0
$$

Equation 20

Where the stoichiometric coefficient $\left(d_{i}\right)$ is positive or negative if it represents a reactant or reagent respectively. As such, a change in molar concentration $\left(d n_{i}\right)$ of species (i)

corresponds to a change of $\left(d n_{i} \frac{d_{j}}{d_{i}}\right)$ in the molar concentration of species $(\mathrm{j})$.

Holding $\mathrm{T}$ and p constant, Seinfeld [7] uses Equation 18 to result in a Gibbs free energy change for the reaction (which is equal to zero at equilibrium) of.

$$
d G=\sum_{j=1}^{k}\left[\mu_{j} \frac{d_{j}}{d_{i}} d n_{i}\right]
$$

Equation 21

At equilibrium ( $d G=0$ ), the following results.

$$
\sum_{i=1}^{k} d_{i} \mu_{i}=0
$$

Equation 22

Which, as noted in [7], can be applied to each reaction that might occur within a system. By using the non-ideal solution expression for chemical potential from Table 2, Seinfeld [7] results in the following equation set which specifies chemical equilibrium.

$$
\prod_{i=1}^{k} \alpha_{i}^{d_{i}}=K_{i}
$$

Equation 23 


$$
K_{i}=\exp \left(-\frac{1}{R T} \sum_{i=1}^{k} d_{i} \mu_{i}^{o}\right)
$$

$\left(K_{i}\right)$ is referred to as the equilibrium constant.

In addition to chemical equilibrium, Gibbs free energy can be used to deduce equilibrium between the gas and liquid phases of a droplet. Important to this deduction is the observation that vapor pressure over any surface increases with curvature. This tendency is called the Kelvin effect. Using the Gibbs free energy associated with a droplet interface of radius ( $\left.R_{P}\right)$, Seinfeld [7] concludes the following expression for the Gibbs free energy change associated with the formation of a particle droplet of surface tension $(\sigma)$ and radius $\left(R_{P}\right)$ from a gaseous state.

$$
\Delta G=-\frac{4 \pi R_{P}^{3} k T \ln S}{3 v_{l}}+4 \pi R_{P}^{2} \sigma
$$

Where $v_{l}$ is the droplet's specific volume. $(S)$ is the ratio of the gas partial pressure in the vicinity of the droplet to the vapor pressure of a flat liquid surface at temperature $(T)$ and is called the saturation ratio.

For $S>1,(\Delta G)$ reaches a maximum, corresponding to an equilibrium point, at a particle radius $\left(R_{P}^{*}\right)$. Seinfeld [7] concludes

$$
R_{P}^{*}=\frac{2 \sigma v_{l}}{k T \ln S}
$$

This equilibrium is only metastable as it represents the maximum for the curve of absolute $G$. Particles perturbed to a radius slightly large than this state will continue to grow. Particles smaller than the equilibrium state, will evaporate to gas.

With Equation 26, the Kelvin equation (relating spherical ( $p$ ) to flat surface $\left(p^{o}\right)$ vapor pressures) can then be found as 


$$
p=p^{o} \exp \left(\frac{2 \sigma M}{k T R_{P}}\right)
$$

Where $\mathrm{M}$ is the molecular weight of the vapor.

\subsubsection{Mechanisms of Particle Evolution}

\subsubsection{Nucleation}

When the partial pressure of a gas's molecules exceeds the vapor pressure of the molecules in a liquid state at the same temperature, super saturation $(S>1)$ is said to exist. Under this condition, a transformation from the gas to liquid phase will occur. The key element in this transformation is the formation of critical liquid molecule clusters through a process called nucleation.

Molecule clusters are continually formed and destroyed, during the life of a gas, due to the random bombardment of gas molecules with each other. When $(S<1)$, these particles rarely exceed what is known as the critical cluster size and will most likely segregate back into individual molecules rather than continue their growth. As the frequency of molecular collisions increases (i.e. partial pressure increases), the chance for larger clusters to exist at any given moment increases. If at any time, the critical cluster size is exceeded, the cluster will most likely continue its growth as Equation 26 suggests. The rate at which these critical clusters are formed is known as the nucleation rate.

Since a molecule may attach itself more readily to foreign matter, nucleation rates can be heightened for multi-component gas systems and / or systems involving foreign material. This phenomenon can be seen as the molecular collision frequency of a gas increases in the vicinity of mutual added attraction and the likelihood of critical cluster 
formation in turn increases. As such, nucleation may occur in these systems for saturation ratios less than one $(S<1)$.

The following describes some results of nucleation theory in its attempt to predict nucleation rates. A more detailed description of this theory can be found in [7].

The classical approach to homogeneous nucleation (i.e. single component gas with no foreign material) assumes steady state cluster generation by the addition or subtraction of single molecules (monomers). In addition, this theory assumes the clusters to be in thermodynamic equilibrium with their surroundings. For these assumptions, it can be shown as in [7] that the nucleation rate $(J)$ is given by.

$$
J=N_{1}\left(\sum_{i=1}^{\infty} \frac{1}{\beta_{i} f_{i}}\right)^{-1}
$$

Equation 28

Where $N_{1}$ is the monomer number concentration, $\beta_{i}$ is the forward rate constant for the collision of monomers with clusters of size $i . f_{i}$ is given by

$$
f_{i}=\prod_{j=1}^{i-1} \frac{\beta_{j}}{\gamma_{j+1}}
$$

Equation 29

Where $\gamma_{j+1}$ is the reverse rate constant for the evaporation of monomers from clusters of size $(j+1)$.

Through the kinetic theory of gases, Seinfeld [7] results in a more meaningful expression for the nucleation rate $(J)$ in terms of the monomer and gas properties, as seen in Equation 30.

$$
J=\left(\frac{2 \sigma}{\pi m_{1}}\right)^{0.5} \frac{v_{1} N_{1}^{2}}{S} \exp \left[-\frac{16 \pi v_{1}^{2} \sigma^{3}}{3(k T)^{3}(\ln S)^{2}}\right]
$$


Where, $m_{1}, v_{1}$, and $N_{1}$ represent the monomer mass, specific volume, and number respectively. The nucleation rate determined by this equation is provided qualitatively in Figure 1, based on the factors of saturation $(S$ ) and temperature (T) involved.

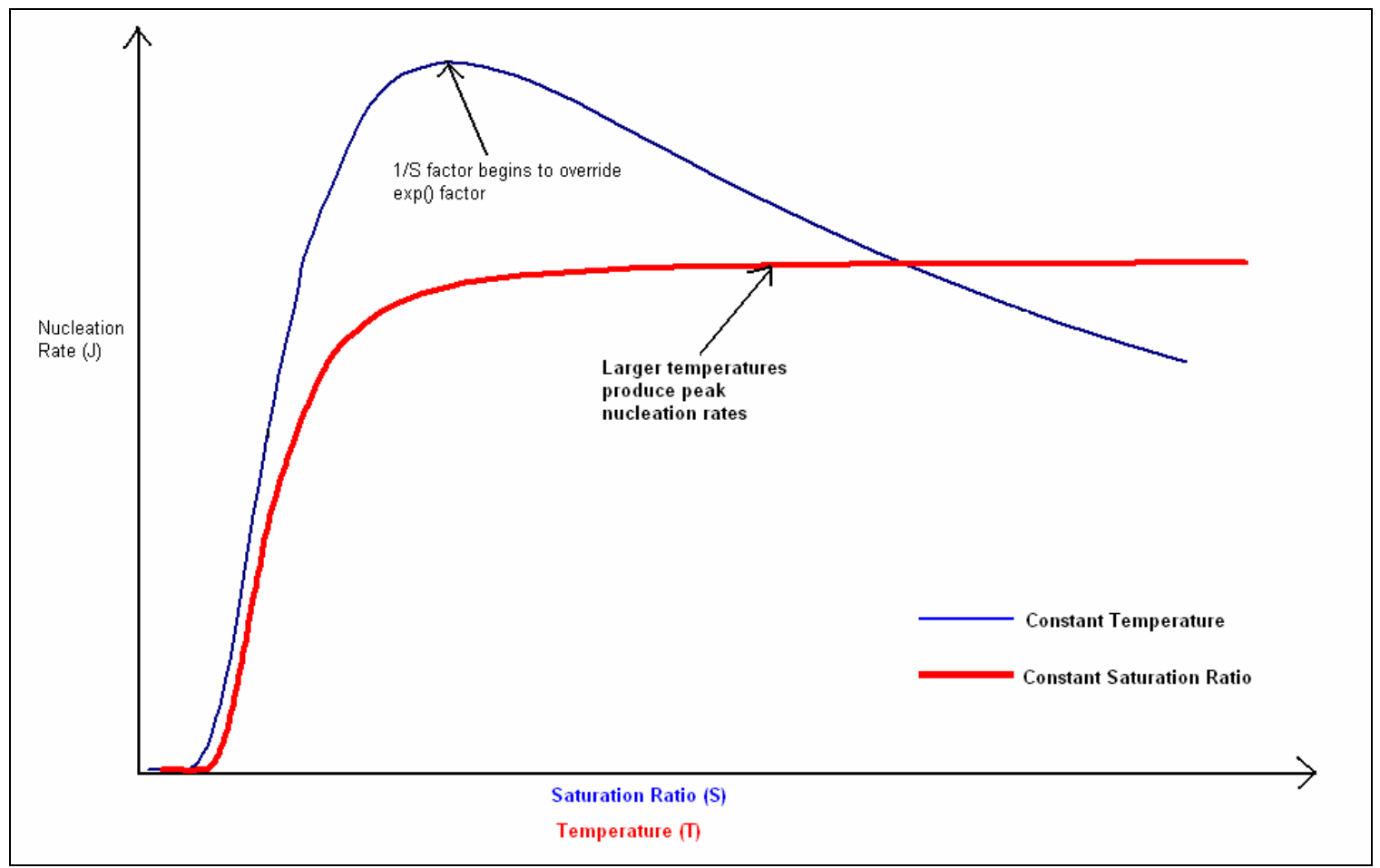

Figure 1 Qualitative Description of the Nucleation Rate Equation [7]

As can be seen in Figure 1, the nucleation rate increases exponentially with saturation ratio $(S$ ) for lower saturation ratios as described by the exponential factor of Equation 30. For larger saturations, the $1 / S$ factor begins to dominate the expression and nucleation rates slowly decline. The nucleation rate has a strong initial dependence on temperature, which levels off at larger temperatures. At lower temperatures nucleation will be extremely inhibited. This might be seen as the temperature being associated with the kinetic energy of the molecule clusters. As such, increasing the temperature increases the likelihood of collisions and therefore critical cluster formation. 


\subsubsection{Condensation}

The theory of condensation describes the processes of evaporation and condensation of monomers to or from aerosol particles. These processes will occur when the partial pressure of the gas is in imbalance with respect to the local particle vapor pressure.

From $[7,45]$ the derivative of a particle's volume with respect to time $(d v / d t)$ is given by.

$$
\frac{d v}{d t}=\frac{2 \pi^{2 / 3}(6 v)^{1 / 3} D_{i} M_{i}}{\rho_{P} R T} f(K n, \alpha)\left(p_{i}-p_{e q u, i}\right) \quad \text { Equation } 31
$$

Where, $\rho_{P}$ is the particle's density. $D_{i}$ and $M_{i}$ are the diffusion coefficient and molecular weight respectively of the gas species. $p_{i}$ and $p_{\text {equ,i }}$ are the partial and equilibrium vapor pressure of the gas species. $f(K n, \alpha)$ is a correction factor due to non-continuum effects, based on Knudsen number and accommodation coefficient $(0 \leq \alpha \leq 1)$

Writing a distribution flux balance, Seinfeld [7] results in the time rate of change of particle number ( $\partial n / \partial t)$ given by

$$
\frac{\partial n}{\partial t}=-\frac{\partial}{\partial v}\left[\frac{d v}{d t} n\right]
$$

Where $(n)$ is the particle number concentration which is a function of volume and time.

Through an example involving constant fluid parameters Seinfeld [7] notes the following:

- Smaller particles grow faster than larger ones under condensation.

- The affected particle size distribution compresses under condensation (i.e. becomes mono-disperse). 
The example provided by Seinfeld [7] offers qualitative information for the initial effects of condensation. These effects will not continue indefinitely as gas properties are affected by the removal of molecules to the liquid state. As the growth of particles within an aerosol continues, the resulting partial pressure of the gas species will be reduced. For large monomer depletions, the partial pressure can be reduced enough to result in the evaporation of monomers from the particles.

\subsubsection{Coagulation}

Coagulation is the process where particles of a given aerosol collide and combine. This occurance will result in an evolution of the aerosol particle distribution. Coagulation can be facilitated by a number of forces including Brownian motion, thermal gradients, and velocity gradients. Coagulation will also be favored by large particle concentrations, since as particle number increases so does the frequency of particle collisions. Therefore dilution processes will act to decrease the rate of coagulation as noted in [5].

The theory of coagulation generally follows statistical attempts to predict the rate of particle collisions. Due to the relative size of coagulating particles, collisions are typically said to result in an absolute unification. The following describes a few results for the theory of this subject.

Through consideration of the Brownian diffusivities of a two particle system consisting of particle diameters $D_{P 1}$ and $D_{P 2}$, Seinfeld [7] concludes a coagulation rate ( $\left.J_{12}\right)$ of

$$
J_{12}=K_{12} N_{1} N_{2}
$$


Where, $\left(J_{12}\right)$ is the collision rate of the two particles per unit volume second. $N_{1}$ and $N_{2}$ are the number concentrations of particles of diameter $D_{P 1}$ and $D_{P 2}$, respectively. $K_{12}$ is the coagulation coefficient for the two particle system given by

$$
K_{12}=2 \pi\left(D_{P 1}+D_{P 2}\right)\left(D_{1}+D_{2}\right)
$$

Equation 34

Where, $D_{1}$ and $D_{2}$ are the diffusivities of the two particle sizes.

In addition to coagulation due to Brownian motion, there is coagulation induced by velocity gradients. Velocity gradients can be formed as a result of shear and / or turbulent flow. For shear flow Smoluchowski [8] demonstrated a coagulation coefficient $\left(K_{\text {coag }}\right)$ of.

$$
K_{\text {coag }}=\frac{\Gamma}{6}\left(D_{P 1}+D_{P 2}\right)^{3}
$$

Equation 35

Where, $\Gamma$ is the velocity gradient perpendicular to the shear direction.

For a rate of dissipation of kinetic energy per unit mass $\left(\varepsilon_{k}\right)$ and viscosity $(v)$, Shaffman and Turner [9] produced a turbulent coagulation coefficient $\left(K_{t u r b}\right)$ of

$$
K_{\text {turb }}=\left(\frac{\pi \varepsilon_{k}}{120 v}\right)^{0.5}\left(D_{P 1}+D_{P 2}\right)^{3}
$$

Equation 36

Seinfeld [7] has noted that the resulting coagulation for velocity gradients is much lower than for Brownian diffusion. This statement is especially true for sub-micrometer particles in turbulent flows. Only under highly turbulent flows do large particles see an appreciable effect from this type of coagulation. 


\subsection{Data Visualization / Interpolation}

Blindly mapping a plume of unknown dimensions and form can require the collection of a massive amount finely positioned samples. A number of these samples may become redundant in determining critical characteristics of the plume. Various visualization schemes have been developed to assist trend observation in multi-dimensional data. These schemes can help hone in on certain diverse regions of data sets and reduce sample point redundancy. As such, using visualization strategies to direct sample locations can significantly reduce the time required to extract important behaviors of a plume.

Many interpolation techniques exist, each having their own advantages and disadvantages. None however, are based on rigorous physical principles. Therefore, the choice of a given technique is never definite. Choosing a technique is generally based on, among other things, smoothness, sample point conformity, and data limitations. Certain schemes may also burden grid selection by placing restrictions on the sampled data. Some techniques require the sample grid to follow a certain structure whereas others require no structure at all.

The list of interpolation methods and their variations is vast and it is not the purpose of this study to discuss them all. As such, the following describes just a few techniques that can be used and were attempted in the visualization of discrete two dimensional data.

These include the one finally chosen for this study.

The first attempt to fit data of a two dimensional grid involved the use of Taylor series. A two dimensional Taylor series has the form.

$$
f(x, y)=\sum_{i=0}^{\infty}\left[\sum_{j=0}^{\infty} C_{i, j} x^{i} y^{j}\right]
$$


A finite version of this equation was used to create a two dimensional polynomial fit to the data. Coefficients $\left(C_{i, j}\right)$ of the polynomial were determined to minimize the total square error with the data set. As an error minimizing technique, interpolations formed from this method are not required to match the sampled data. In addition, while contours generated by this technique appeared smooth, they failed to provide a "realistic" representation of the data. For higher order data sets, variations tended to be highly exaggerated at best. In certain specialized cases (data sets) fits seemed adequate but always failed to match the data closely. As such, the technique was determined not stable enough for the interpolation of general data sets which might occur in the mine.

The second attempted technique involved a structured grid and falls under the category of finite element interpolation. Non-structured grids may also be found in this category but require a little more preconditioning to achieve interpolation. In this technique the data samples are used to create the nodes of cell elements which are interpolated locally. The simplest case involves a square cell. In this case, linear interpolations are made across two opposite sides of the element as shown in Figure 2. 


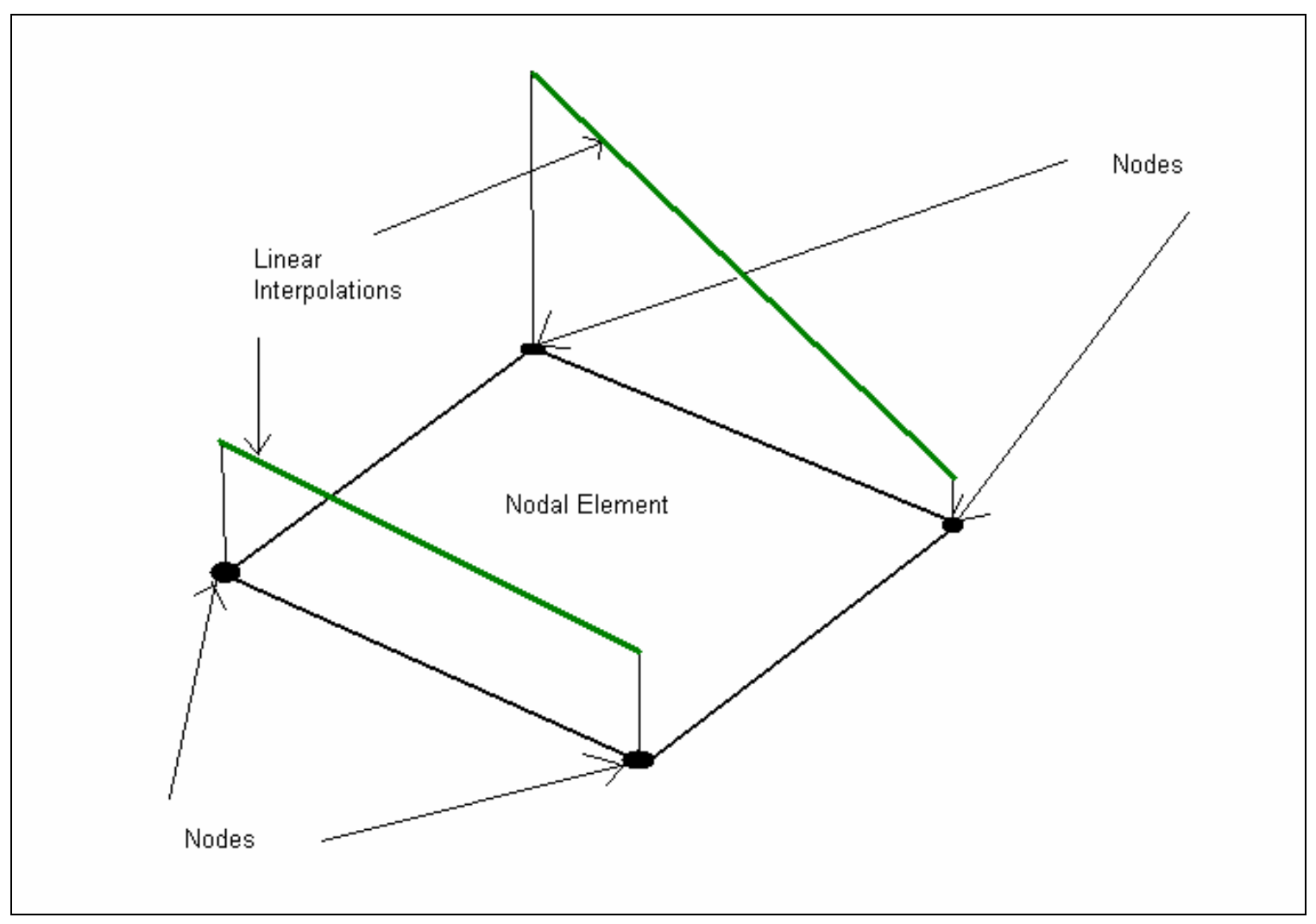

Figure 2 Preliminary Linear Interpolations for a Square Element

The interior is then completed with a series of linear interpolations which use the first two interpolations as end points. Figure 3 shows an example of an interior interpolation through the center of the cell of Figure 2. 


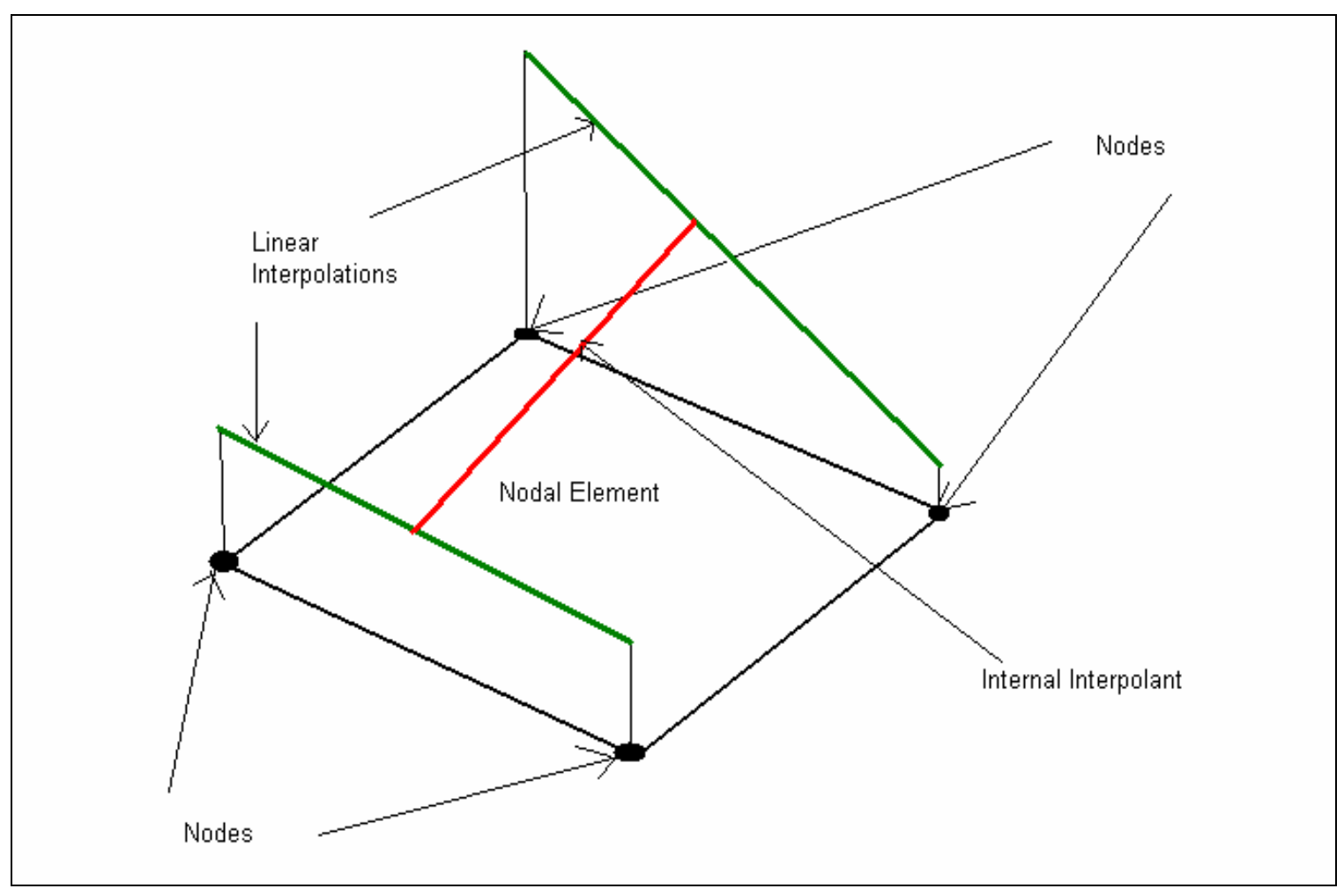

Figure 3 Example Internal Interpolation for Square Element

This method is guaranteed to match the sampled data but lacks in the smoothness that is sometimes beneficial when viewing trends. The method is made smoother by using higher order fits for the first two interpolations. However, extra information needs to be supplied at the nodes to make the resulting equation set complete. One higher order example uses a third order fit. This is called Hermite-Linear interpolation. The extra information consists of four nodal derivatives in the directions of the first two interpolations. This technique smoothes the data considerably but was found highly sensitive to the selection of nodal derivatives. In addition, smoothness is only guaranteed in the nodal derivative directions. For these reasons, the method was not chosen for this study.

The last interpolation technique that will be discussed is also the one chosen for this study. It is found in "A Comparison and Evaluation of Interpolation Methods for 
Visualizing Discrete 2D Survey Data” [10]. In this method scattered data comprised of $N$ sample points is fit by a function $f(X)$ through the use of radial basis functions. $f(X)$ has the following form [10].

$$
f(X)=\sum_{i=1}^{N} \alpha_{i} R\left(d_{i}(X)\right)+p_{m}(X)
$$

Equation 38

Where

$$
p_{m}(X)=\sum_{i=1}^{m} \beta_{i} p_{i}(X)
$$

Equation 39

In these equations $X$ is a coordinate vector, $d_{i}$ is the distance from coordinate $X$ to sample point coordinate $X_{i}$ and $R$ is a univariate basis function of $d_{i} \cdot p_{m}(X)$ is an $m^{\text {th }}$ degree polynomial guaranteeing exact reproduction of $f(X)$ for $\left(\alpha_{i}=0\right)$ [11].

To determine the coefficients of $f(X)$ and $p_{m}(X)$, the following constraints are imposed, which form a linear system of equations.

$$
\begin{aligned}
& f(X)=f_{i} \quad \text { for } \quad i \in(1, \ldots, N) \\
& \sum_{i=1}^{N} \alpha_{i} p_{j}\left(X_{i}\right)=0 \quad \text { for } \quad j \in(1, \ldots, m)
\end{aligned}
$$

Equation 40

Equation 41

As done in [10], to help keep the condition of the linear system acceptable the radial basis function was chosen as:

$$
R\left(d_{i}\right)=d_{i}^{2} \log \left(d_{i}\right)
$$

Equation 42

This function is said to minimize the bending energy of a thin plate forced through the sample points and is known as Duchon's Thin Plate Spline [12].

The resulting interpolations of this method were found to be smooth and visually agreeable with a wide variety of data. Do to the fact that data points of this technique are not required to follow any structure and do not require the generation of cells, 
programming was relatively simple to develop. In addition, as per the boundary conditions, interpolations are forced to the sample points. For these reasons, this method was chosen to be used for the study.

Care should be taken in the use of interpolation techniques, as they are not strictly based on science. They are merely a tool which can be valuable in directing scientific interests. As trends in certain regions of data are observed, it is important to refine these regions to check for visual or numerical convergence. If further agreement is observed, the trend is still only highly probable at best, as artifacts are present in every interpolation scheme.

One possible reason for the observance of artifacts in an interpolation scheme is when the coordinate vector $X$ becomes inhomogeneous. In this situation, some form of dimensional scaling must be used in calculating the coordinate distances $d_{i}$. This can become an issue as the inherent dependence of the scalar field $f$ may be non-uniform across the coordinate elements. As such, scaling can become an issue even in the homogeneous case. The following example demonstrates this effect.

Consider the rectangular shaped intensity of Figure 4 to be mapped by the fine resolution lines L1 and L2 through radial basis functions. 


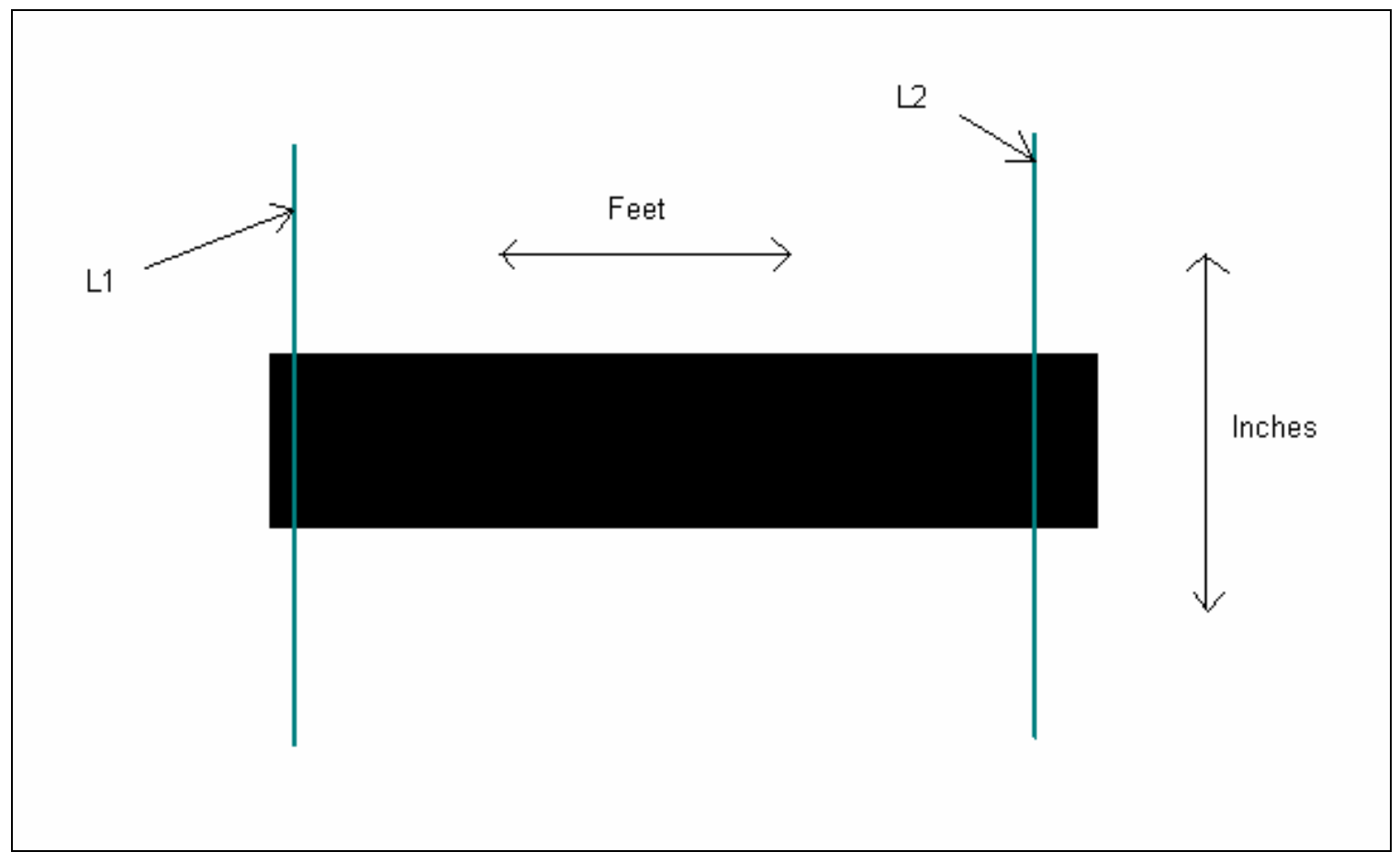

Figure 4 Rectangular Shaped Intensity to be Mapped Through Lines L1 and L2

The dimensions are homogeneous in the fact that they represent lengths. However, the horizontal distance is listed in feet while the vertical distance is listed in inches. If the horizontal dimension is merely converted to inches and the intensity mapped by radial basis functions, the following might occur. 


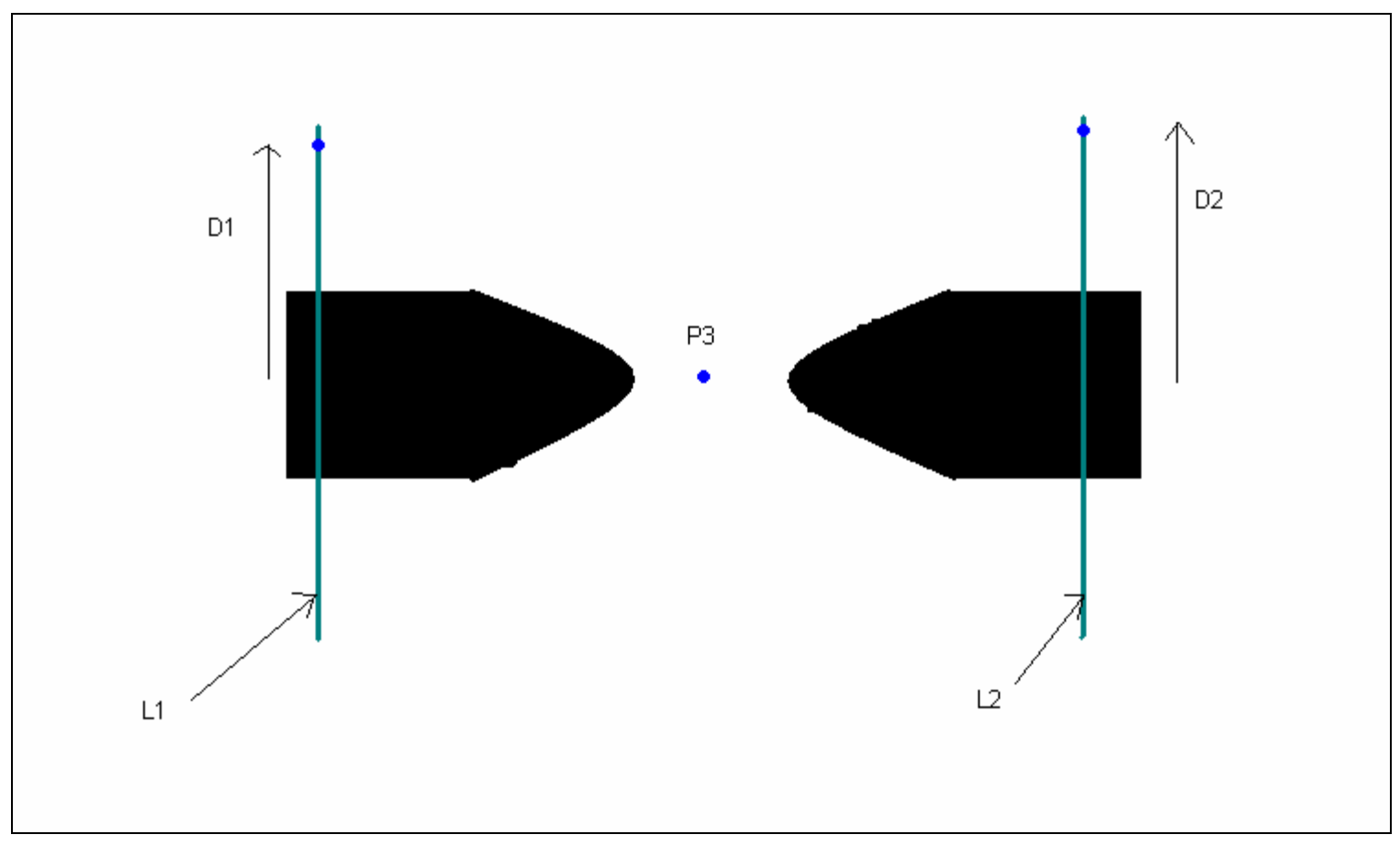

Figure 5 Distorted Mapping Caused by Dissimilar Dimensions

As can be seen, the regions of the rectangle close to the lines L1 and L2 are mapped well. However, the center region of the rectangle near point $\mathrm{P} 3$ has become cut in two. This can be seen by the fact that the distance from P3 to all points on L1 and L2 is similar to the distances D1 and D2 respectively. As such, each contribution of L1 and L2 to point P3 is similar to a point outside of the rectangular intensity.

This issue can be resolved by scaling down the horizontal dimension for distance calculation purposes. The region can then be stretched back to its original size during the map generation process. In cases where the form of the region to be mapped is unknown, scaling coordinate dimensions can become more complex. 


\subsection{Instrumentation}

The following sections provide details concerning some of the critical instruments used and built within this study. Other instrument and software descriptions will be provided as necessary in Section 3.

\subsubsection{Scanning Mobility Particle Sizer (SMPS)}

The Scanning Mobility Particle Sizer (SMPS) is an instrument developed by TSI Incorporated used to measure the steady state particle size distribution of an aerosol. This instrument has a particle detection limit of 5 to 1000 nanometers.

The SMPS works by first charging particles and then sorting them through the use of a varying electric field. The sorted particles are then grown and counted in a Condensation Particle Counter (CPC). This process is automated through serial communication software control (Aerosol Instrument Manager) of the CPC or SMPS. A complete description of SMPS operation is provided in TSI's instruction manual [17].

\subsubsection{Model 3007 Condensation Particle Counter}

The Model 3007 Condensation Particle Counter (CPC3007) is a handheld particle counter used in the measurement of aerosol concentrations. Concentration measurement ranges are limited to 100,000 particles per cubic centimeter. A complete description of CPC3007 operation is provided in TSI's instruction manual [18].

\subsection{West Virginia University Wind Tunnel Study}

A study similar to the one presented here was conducted by West Virginia University (WVU) in cooperation with the Desert Research Institute (DRI) and the University of 
Minnesota (UMN) [43]. This study also sought the characterization of PM originating from an on-highway diesel engine, but from a Class-8 heavy duty diesel truck operating outside any mine environment. Tests were performed in the Old Dominion University Langley full-scale wind tunnel. Wind tunnel speeds of $88 \mathrm{~km} / \mathrm{hr}$ were achieved with the vehicle operating at $88 \mathrm{~km} / \mathrm{hr}$ and the engine operating at at $2200 \mathrm{RPM}$ and $406 \mathrm{ft}-\mathrm{lb}$ [43]. As in the current study, scalar exhaust mapping of $\mathrm{CO}_{2}$ were performed prior to aerosol sampling so that the plume's edge and centerline could be indentified. Durring the study, WVU responded to many issues including nuclei mode contamination from nearby aircraft, outer plume measurement error, and effects of the recirculation tunnel on background measurements.

Ultimately, WVU concluded no detectable nuclei mode under any engine setting other than idle. This observance was attributed to lower exhaust temperatures and higher saturation ratios [43]. In addition, aerosol measurements taken throughout the plume showed that dilution scaling was the only source for aerosol size distribution dissimilarity throughout the plume. These results were also highly varied when compared to similar engine studies of trucks operating on the open road and suggest that there may be a cause from the plume’s environment. 


\section{Experimental Layout and Procedures}

This study aimed for the thorough yet efficient acquisition of diesel exhaust data on aerosol formation in mine environments. The process of designing test procedures for this study was evolutionary and involved some preliminary work. The following section describes the test environment and experimental procedures used during this study. In an effort to aid this discussion, some preliminary results will be shown. In addition, potential issues that might jeopardize the results of this study will be demonstrated through qualitative examples.

\subsection{Test Environment (D-drift)}

\subsubsection{General}

The experiment part of this study was conducted in an underground tunnel (D-drift). D-drift is a part of Lake Lynn Laboratory (LLL), a research mine facility established by the National Institute for Occupational Safety and Health (NIOSH) [20]. In addition to an understanding of diesel exhaust aerosol formation and evolution in mine environments, tests performed here have the added benefit of establishing fully mixed conditions for the tunnel. Fully mixed conditions are to be achieved, by researchers of the mine, for the evaluation of bulk emissions production in mine environments. The overall layout of D-drift is provided in Figure 6. 


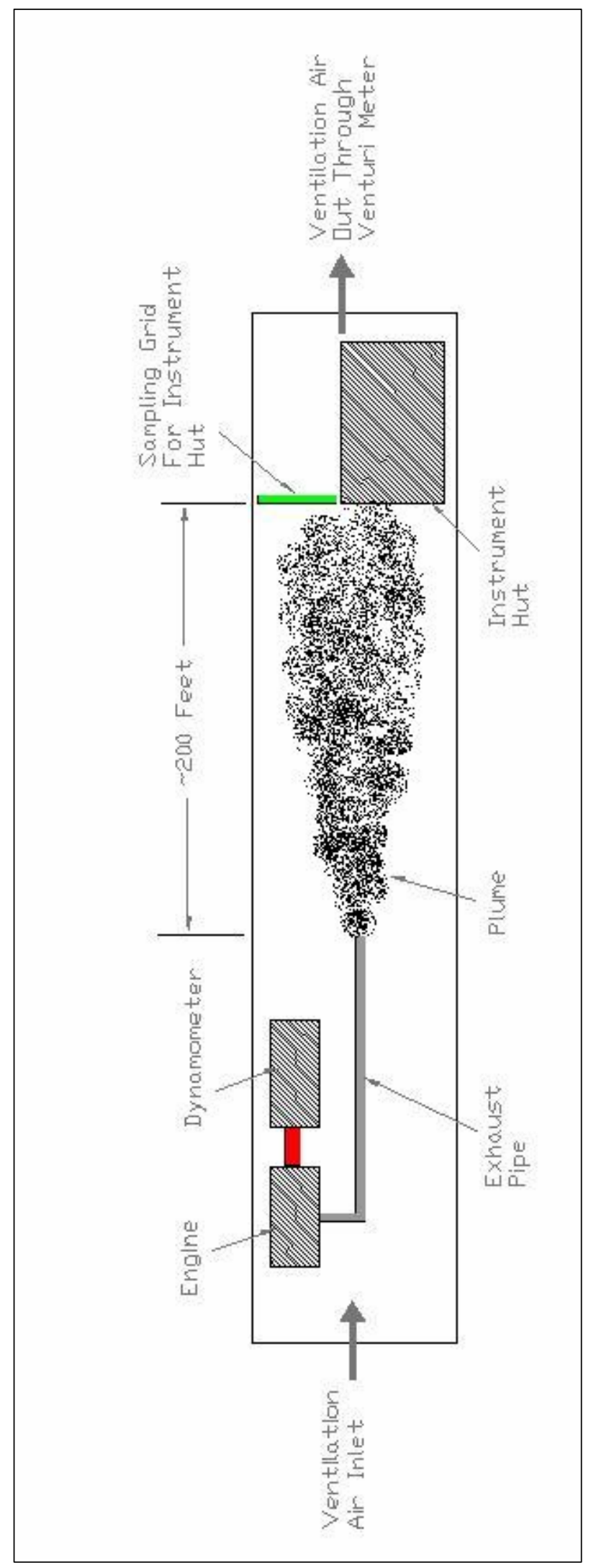

Figure 6 D-drift General Arrangement 
The section of D-drift, chosen to be studied, spans the roughly 200 feet between the engine / dynamometer system and instrument hut. The tunnel cross-section in this region is approximately 19 feet wide and 7 feet tall. Tunnel dimensions can vary by more than a foot in certain regions (primarily in width), but no attempt was made to map these variations due to their highly irregular nature. The tunnel sloped producing a roughly 4 foot drop in elevation for every 100 feet of travel in the exhaust flow direction. Figure 7 provides an image of the tunnel taken near the engine in the direction of the instrument hut.

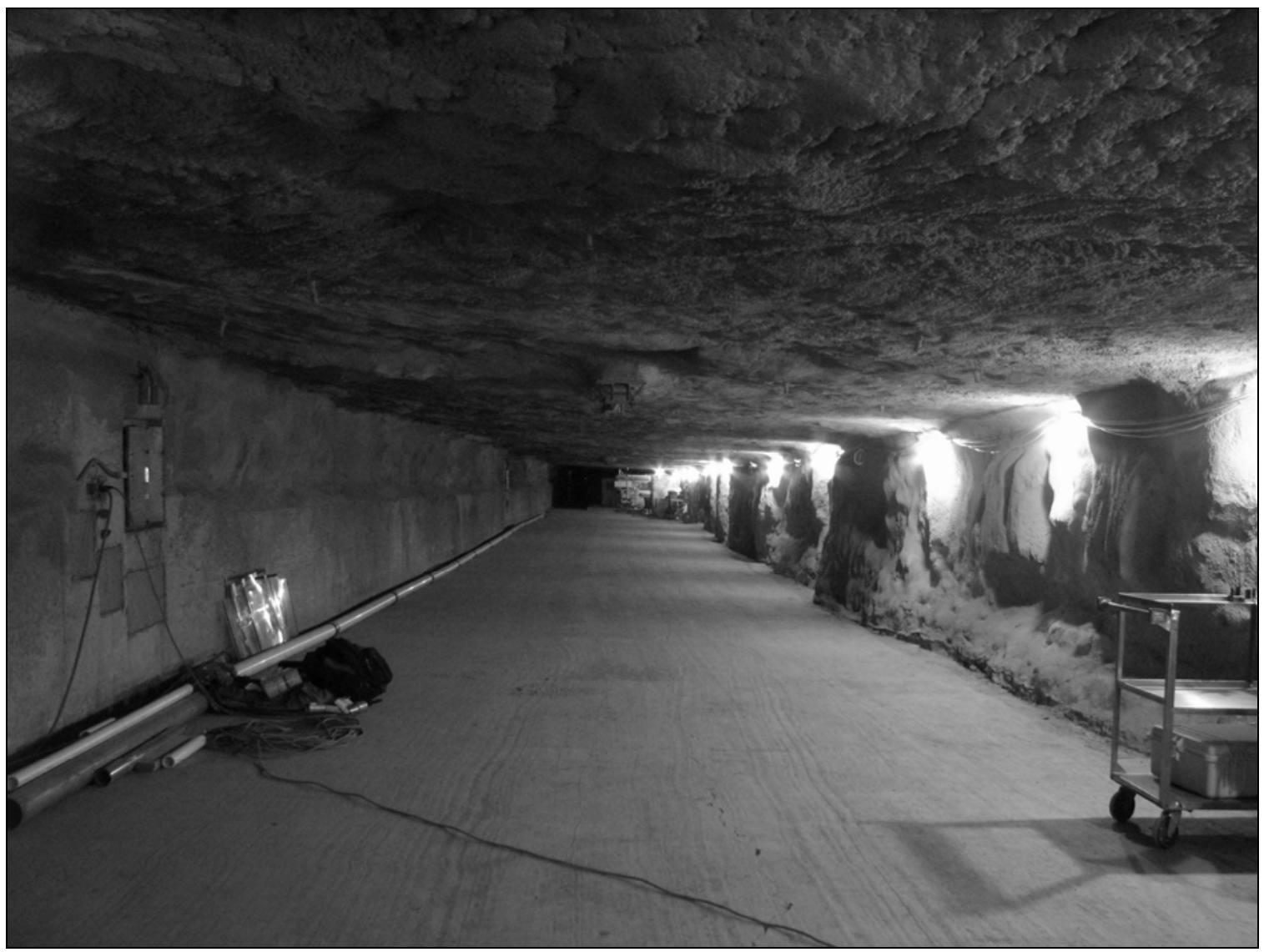

Figure 7 Image of D-drift Tunnel Test Section

Figure 7 demonstrates the geometrical variations present in the tunnel. However, the tunnel is mostly rectangular with the bulk of its variation present on the right wall. In 
addition, the ceiling and floor both slope to the right slightly and are much less variant geometrically.

\subsubsection{Engine / Ventilation}

The engine used in this study was an Isuzu C240 with 300 hours of operation since new. This engine was naturally aspirated, mechanically controlled, and operated on regular low sulfur fuel ( $\sim 300 \mathrm{ppm})$ throughout the study. Further descriptions of this engine and its operation can be found in [13]. A summary of the key characteristics of this engine is given in Table 3.

Table 3 Key Characteristicss of Isuzu C240 [13]

\begin{tabular}{|c|c|}
\hline Engine Type & $\begin{array}{c}\text { 4 Cylinder, 4 Cycle, Water Cooled, OHV } \\
\text { Indirect Injected }\end{array}$ \\
\hline Bore and Stroke & 3.39 x 4.02 inch \\
\hline Piston Displacement & 2.4 liter \\
\hline Compression Ratio & $20: 1$ \\
\hline Coolant Capacity & 5.2 liter \\
\hline Lube Oil Capacity & 4.3 liter \\
\hline Rating (Intermittent) & 56 BHP @ 3000 RPM \\
\hline Rating (Continuous) & 49 BHP @ 3000 RPM \\
\hline Rating (Maximum Torque) & 93 lb-ft @ 2000 RPM \\
\hline
\end{tabular}

Two modes of engine operation were chosen for this study. These modes were rated 75\% (R75) and intermediate 30\% (I30). Table 4 provides the operating conditions associated with each of these engine modes. 
Table 4 Relevant C240 Modes of Operation Characteristics

\begin{tabular}{|c|c|c|}
\hline Engine Set Point & Torque (ft-lbs) & Engine Speed (RPM) \\
\hline R75 & 70 & 3000 \\
\hline I30 & 27 & 2000 \\
\hline
\end{tabular}

The exhaust system, for the C240, was constructed of 3 inch pipe with sample ports for the collection of raw $\mathrm{CO}_{2}$ concentration, backpressure, and temperature. The outlet of the exhaust was centered and aligned with the tunnel.

Such issues of exhaust alignment might play a critical role in the repeatability of testing results but are less likely resolved in actual working use. It is unlikely that an engine operator would be able to perform their job or even benefit by maintaining an exhaust that is centered and aligned with the tunnel. Nevertheless, in order to maintain some form of consistency, the exhaust was centered and aligned with the tunnel and consequently the ventilation air as well.

Ventilation air entered the tunnel from a ventilation shaft far ahead of the engine (500+ feet) and exited through a venture meter installed at the end of the tunnel located far downstream the instrument hut (500+ feet). The inlet air was drawn through a vertical shaft from outside the mine and was conditioned prior to entering the tunnel to remove moisture. Flow rate $(\sim 10,000 \mathrm{cfm})$ was recorded each day from measurements made at the venturi. Background air properties such as $\mathrm{CO}_{2}$ concentration, temperature and humidity were also recorded daily. 


\subsection{Test Apparatus}

The test apparatus (see Figure 10) was designed as a cost effective means to accurately and efficiently position sample probes within various cross sectional locations of the plume. Continuous cross sectional scans can be made through a square 84 by 84 inches of horizontal and vertical travel respectively, which encompasses the height of the mine tunnel studied here. These scans were routinely integrated into accompanying computer software allowing up to date imagery of current probe position projected on interpolated predictions of future readings. This aids the user in the selection of points of interest and diminishes the time needed to capture important characteristics of the plume. Various scalars such as temperature, $\mathrm{CO}_{2}$, and aerosol concentration can be used as a basis for plume prediction. In addition to their procedural necessity, multiple measures, at the same location, were used to identify potential problems in measurements.

The probes were matched to a single set of instruments and each probe position represented a single point (not an array). Therefore scans were time averaged and instantaneous two dimensional plume mappings could not be made. In spite of this, plume fluctuations could be acquired from the data associated with each point used to produce an average mapping.

The test apparatus was designed to minimize the length of the PM sampling lines by placeing the aerosol instrumentation close to the probes. SMPS and CPC instrumentation were placed in protective cart which carried the instruments throughout the plume. Sample probes extend out of a metallic shield, which protects the instrumentation from hot exhaust, located on the front of the cart. A frontal view of the shield and probes is provided in Figure 8. 


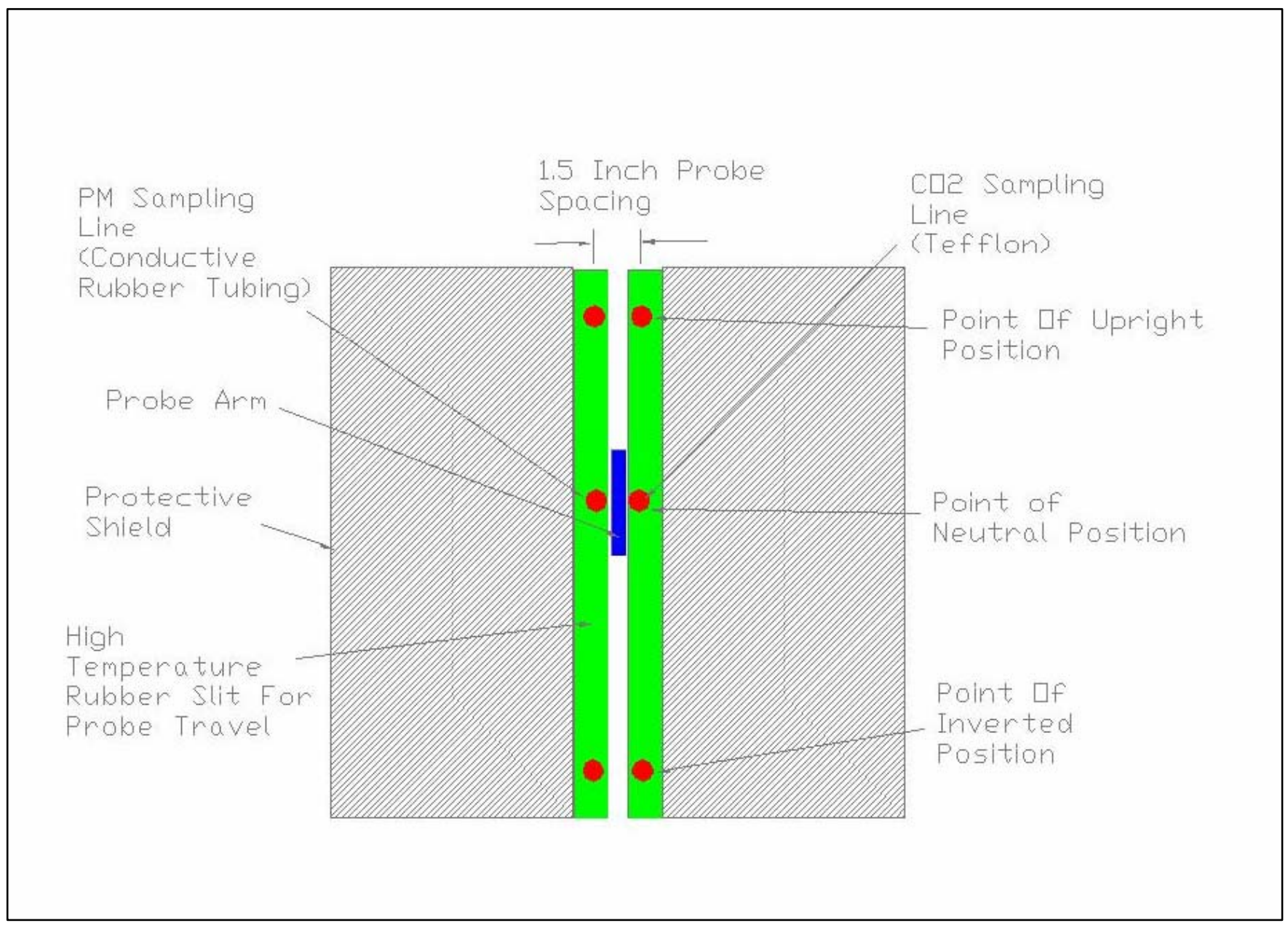

Figure 8 Frontal SMPS Shield Showing Various Probe Arrangements

As can be seen in Figure 8, the probes can be attached to the probe arm in one of three orientations (upright, neutral, or inverted). The probe arm will rise and fall inside the rubber slit as the cart travels up and down respectively. Conductive rubber tubing is used for the PM probe sample line to reduce particle loss.

The dimensions of the shield are 18inches wide by 22inches tall with the apex extending forward $\sim 18$ inches. Beyond the apex, the 1/4inch diameter probes extend an additional 12 inches. Studies (either experimental or computational) should be performed to determine the flow interference caused by this shielding. However, since the length of the shield and probes is $\sim 2$ times the width of the shield and the flow speed was 
$\sim 40 \mathrm{ft} / \mathrm{min}$, interference was not expected to be significant in the measurement of aerosols. A study performed by Norment [44] showed that a probe design of roughly 7inches wide by 40inches long resulted in an undersampling of particle diameters greater than $20 \mu m$ by $\sim 10 \%$. However, in Norment's study the probe system was attached to an aircraft traveling at $\sim 225 \mathrm{~m} / \mathrm{s}$. With the lower speeds found in the tunnel of this study, sampling interference should be far less than in [44].

The test apparatus was designed to allow complete operation outside of the plume. This reduced operator exposure to high concentrations of gases and particles in the core of the plume. In addition, overall operation efficiency was increased as all controls were located in one place.

The basic design of the test apparatus can be divided in two sections, a moveable horizontal platform connected to a vertical tower containing the instrument platform, SMPS, CPC, and probes. The following describes the assembly of the horizontal platform. 


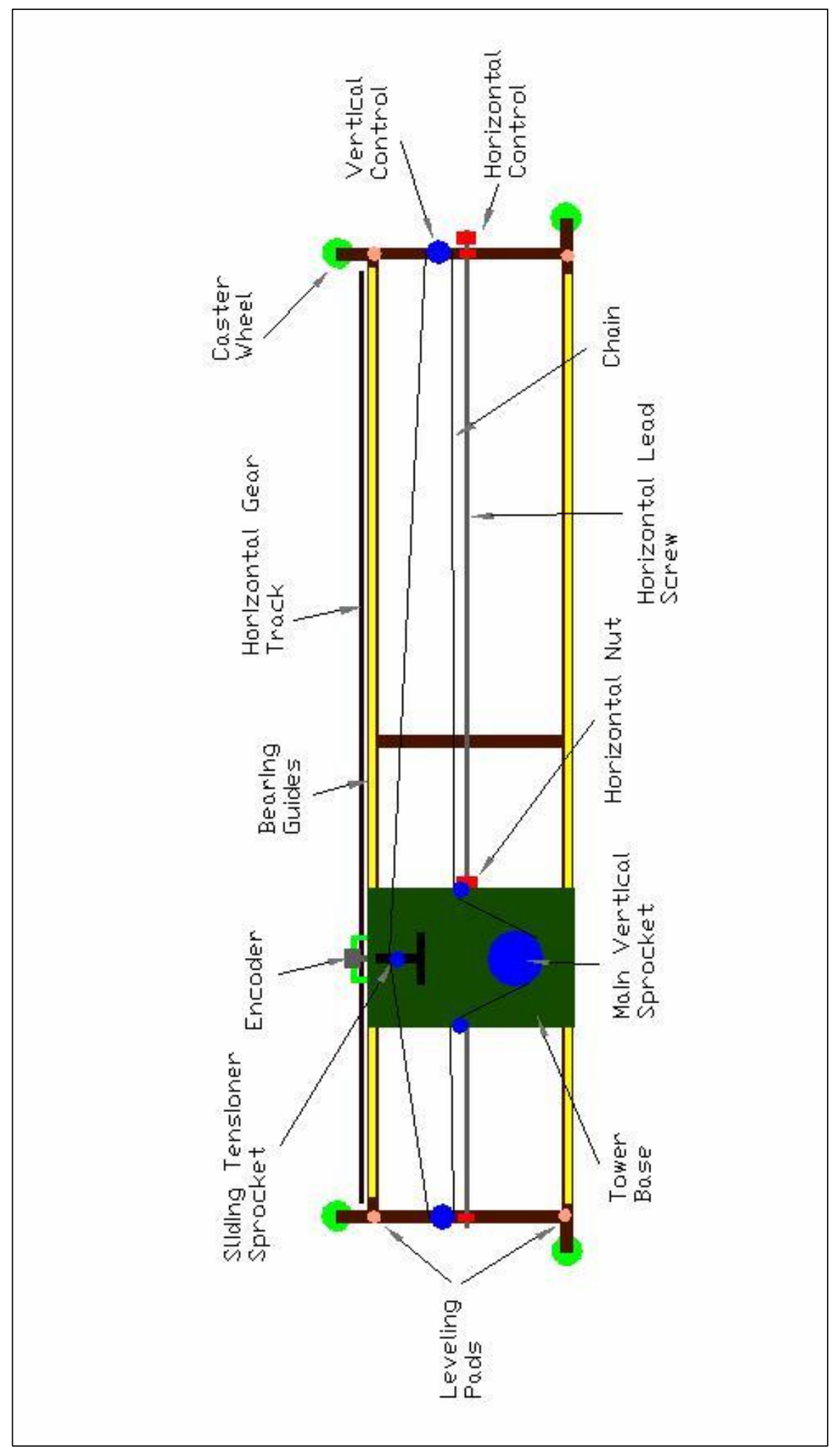

Figure 9 Test Apparatus Horizontal Drive Assembly 
As it can be seen in Figure 9, the tower base rests on four bearings situated on two tracks. Horizontal motion is transferred to the base by an attached nut threaded to the horizontal lead screw. Digital positioning of the tower base is obtained by a Koyo TRDN60 encoder from AutomationDirect, which is mounted to the tower base and driven across the horizontal gear track. Axial motion is assisted through four caster wheels located at the corners of the horizontal platform. Once an axial station is reached, the leveling supports are driven down past the wheels into removable bearing platforms. Vertical motion is transferred to the main vertical sprocket, which is connected to a vertical lead screw, by way of chain. The vertical tower (shown in Figure 10) is attached to the tower base. 


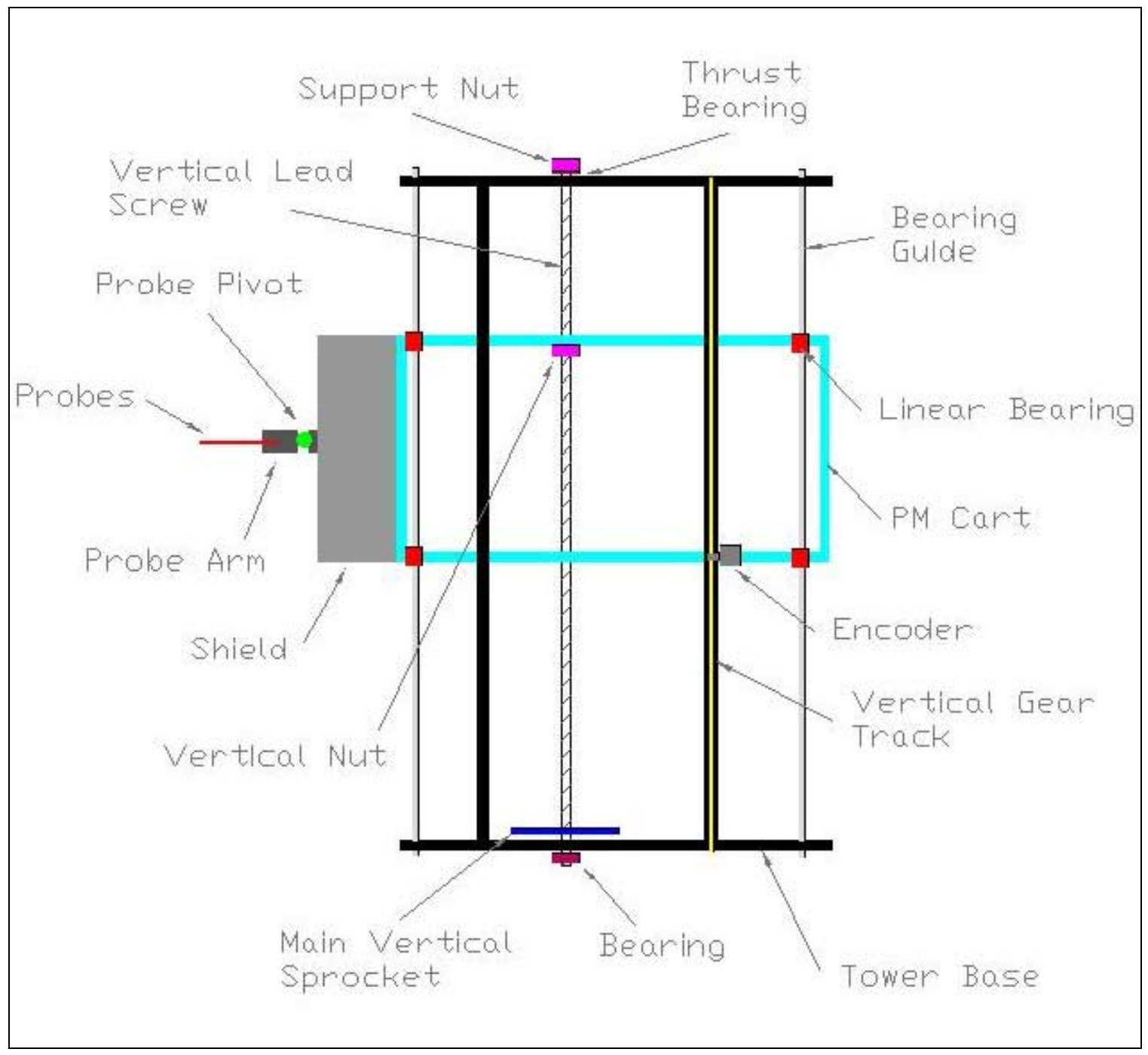

Figure 10 Vertical Tower Assembly

As can be seen in Figure 10 the vertical tower consists of an outer frame supporting the inner instrument platform. The weight of the cart and instrumentation is supported by an attached nut connected to the vertical lead screw. The vertical lead screw is hung from the top of the outer frame on a thrust bearing and is driven below by the main vertical sprocket. Four bearing tracks guide the cart through its travel. To prevent the cart from binding, the guide bearings are mounted in two planes (each plane contains four bearings). The position of the cart is obtained through an encoder, which is mounted to the cart and driven across the vertical gear track. Due to the height of the instrument cart, 
effective travel of the sample probes is limited. To resolve this issue, an inner gear system, supporting the sample probes, was attached to the cart. This system is shown in Figure 11.

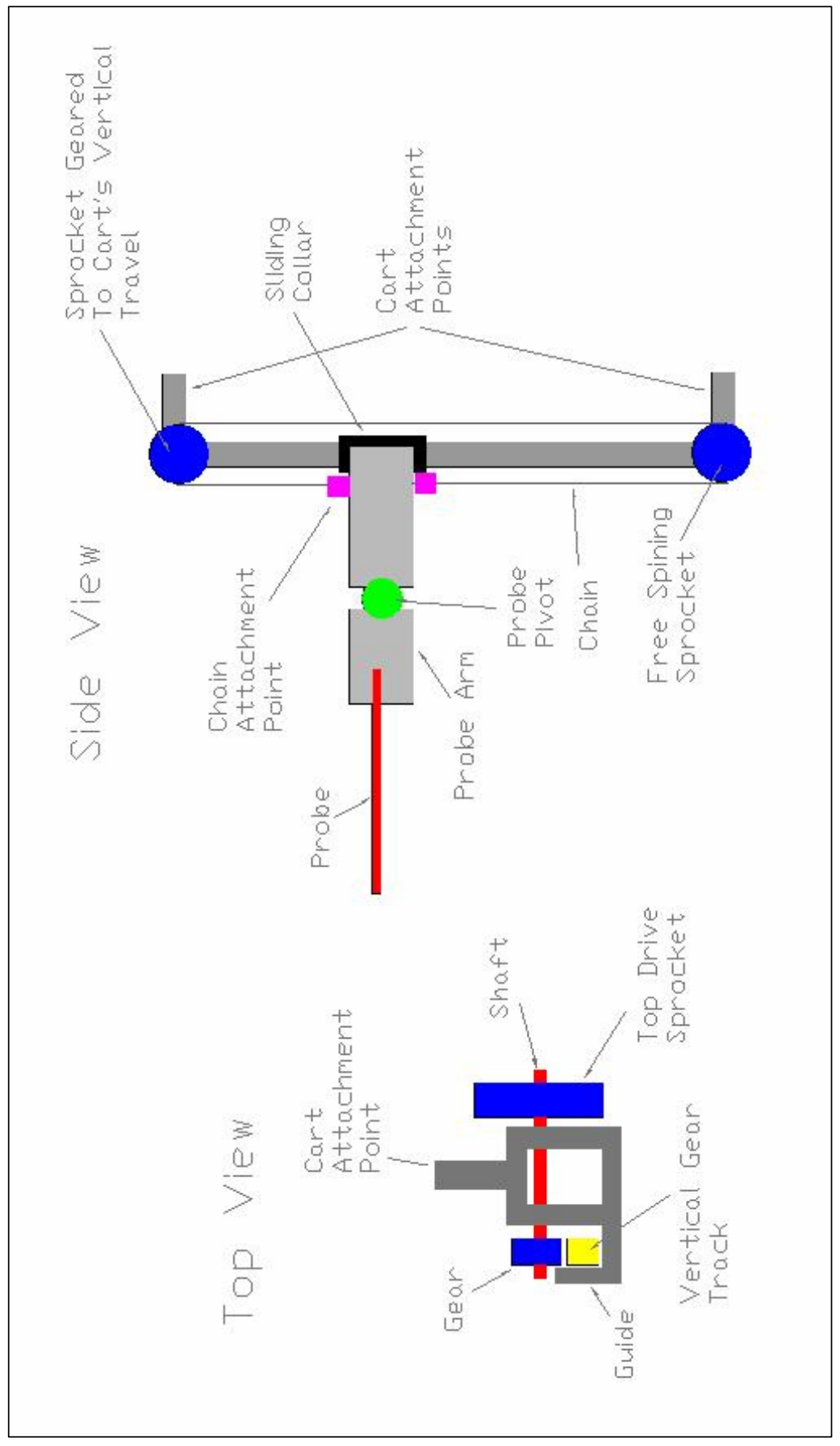

Figure 11 Inner Vertical Gear Assembly 
This system mounted within the shield and continuously raised the probe arm to the top and bottom of the cart as the cart has reached its peek and minimum height respectively. The probe arm extended out of a high temperature rubber slit shown on the protective shield of Figure 8. Since the gearing is linear, encoder output from the cart could easily be used to determine probe elevation. A shifting probe was also made to allow scanning in the upper and lower regions of the tunnel. This allowed a potential 27 more inches of travel.

Figure 12 shows the test rig in its fully assembled form, as it was set up in the mine.

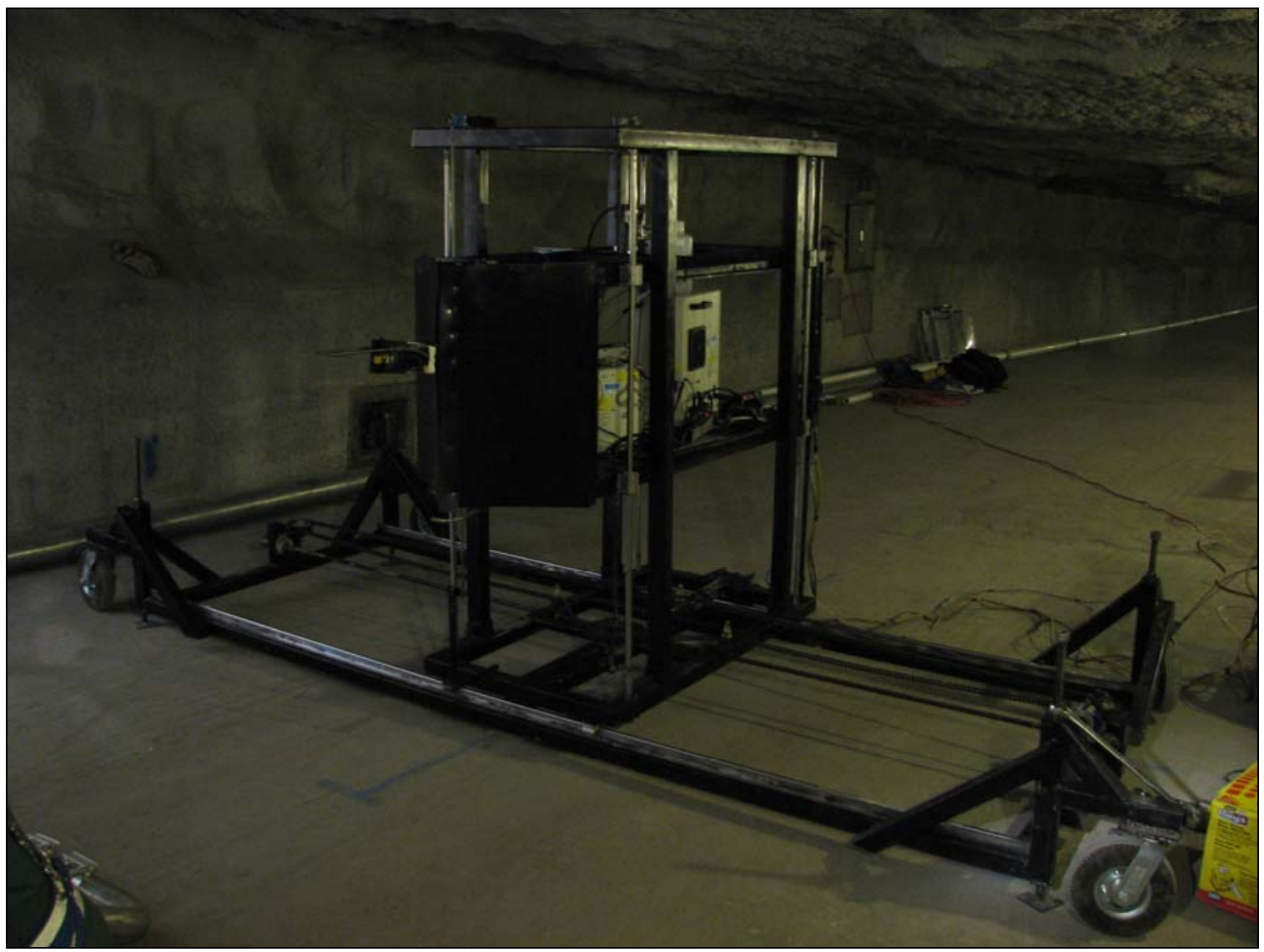

Figure 12 Fully Assembled Test Rig 


\subsection{Methodology}

\subsubsection{General}

The approach employed in this study involved using precise scalar exhaust maps of average $\mathrm{CO}_{2}$ concentration, temperature, and aerosol concentration to strategically acquire more time exhaustive samples of aerosol size distribution. This was an effort to extract comprehensive aerosol formation and evolutionary trends without the burden of over sampling (i.e. tracking symmetrical and contoured aerosol trends). In addition, completeness in the resulting trends can be argued by their relative similarity and placement within the scalar exhaust maps.

The study began by dividing the 200 foot test region into a number of axial stations where cross-sectional exhaust maps of $\mathrm{CO}_{2}$ concentration, temperature, and aerosol concentration were made (mapping was based on the radial basis function explanation of Section 2.3). The axial placements of the stations were chosen over a preliminary four day test period, with the engine operated at rated speed and 75\% load (R75), to capture critical plume behavior within the test region. Each station resulted in a set of precise cross-sectional scalar exhaust maps, demonstrating regions of high and low plume influence, to be used as a guide for aerosol size distribution sample placement. Figure 13 describes the general axial placement of the stations as they relate to critical spatial features of the plume. 


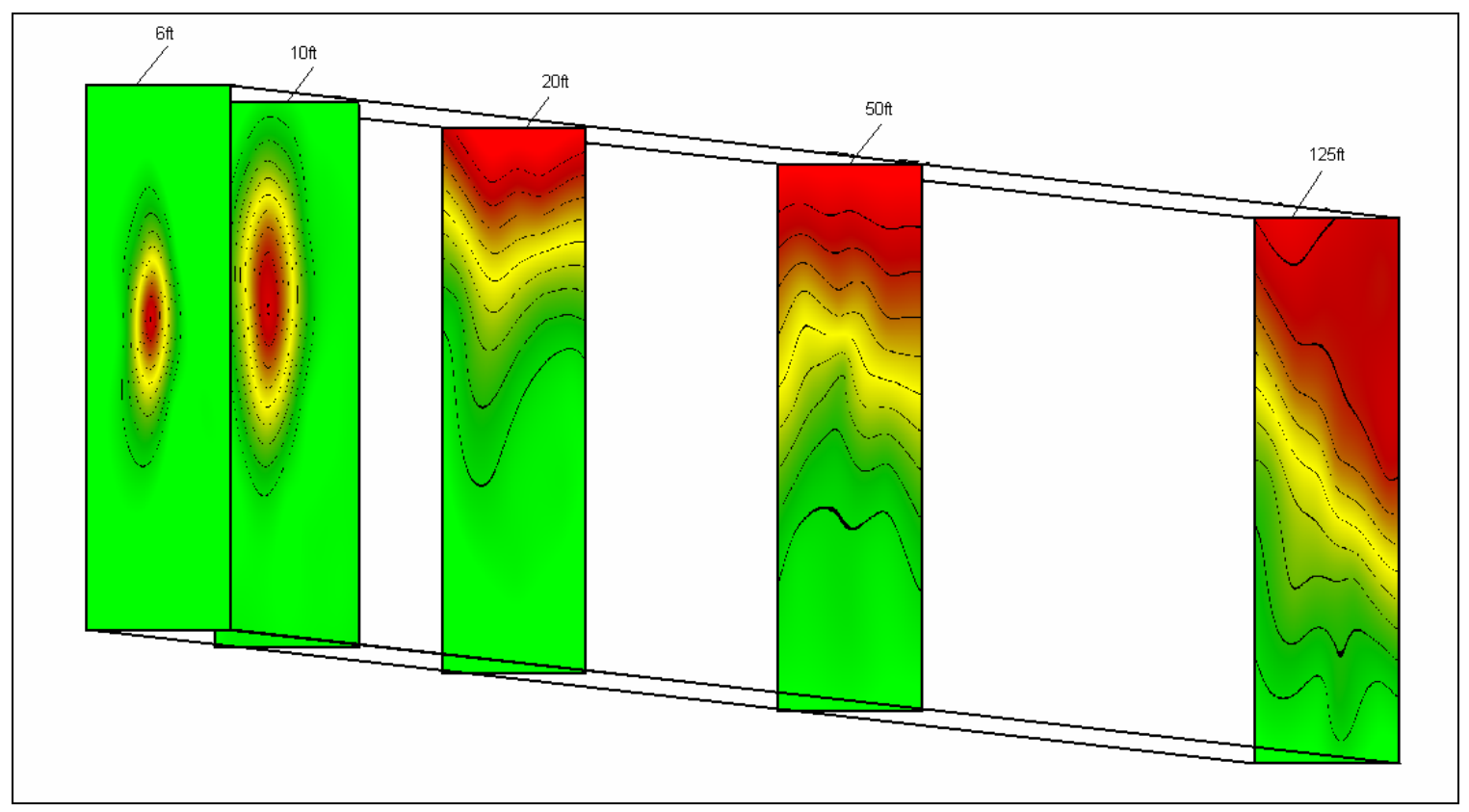

Figure 13 General Axial Placement of Cross-sectional Maps (Contour levels are not drawn to the same scale for each station and are given only to suggest the geometry of the plume.)

The scale used in Figure 13 is not uniform across each station and is given only to suggest the shape of the emerging plume. In reality, the scale of downstram stations dwarfs any upstream scales. As can be seen, the plume existed in concentric form up to roughly 10 feet. After that, contact with the ceiling (due to buoyancy and tunnel slope) began to flatten out the plume into stratified layers. Beyond 50 feet, only minute spatial variations were observed in the plume (other than differences in the orientation of stratification lines). The waviness present in downstream stations may have been triggered by a number of environmental influences including local tunnel geometry. These were resolved through larger averaging times for the more distant axial stations. Typically however, single point averages were found adequate after only 1 minute of sampling at $1 \mathrm{~Hz}$.

Some adjustments were made to the axial locations shown in Figure 13 (i.e. when the engine was operated at Intermediate 30\% (I30)) and were recorded later. However, 
general spatial characteristics of the plume remained unchanged in spite of large differences in engine set point.

The scalar exhaust maps were acquired by interpolating a number of single point averages taken at each axial station. Figure 14 demonstrates an example $\mathrm{CO}_{2}$ map taken at the 10 foot station on day 7, with the engine operating at R75 conditions.

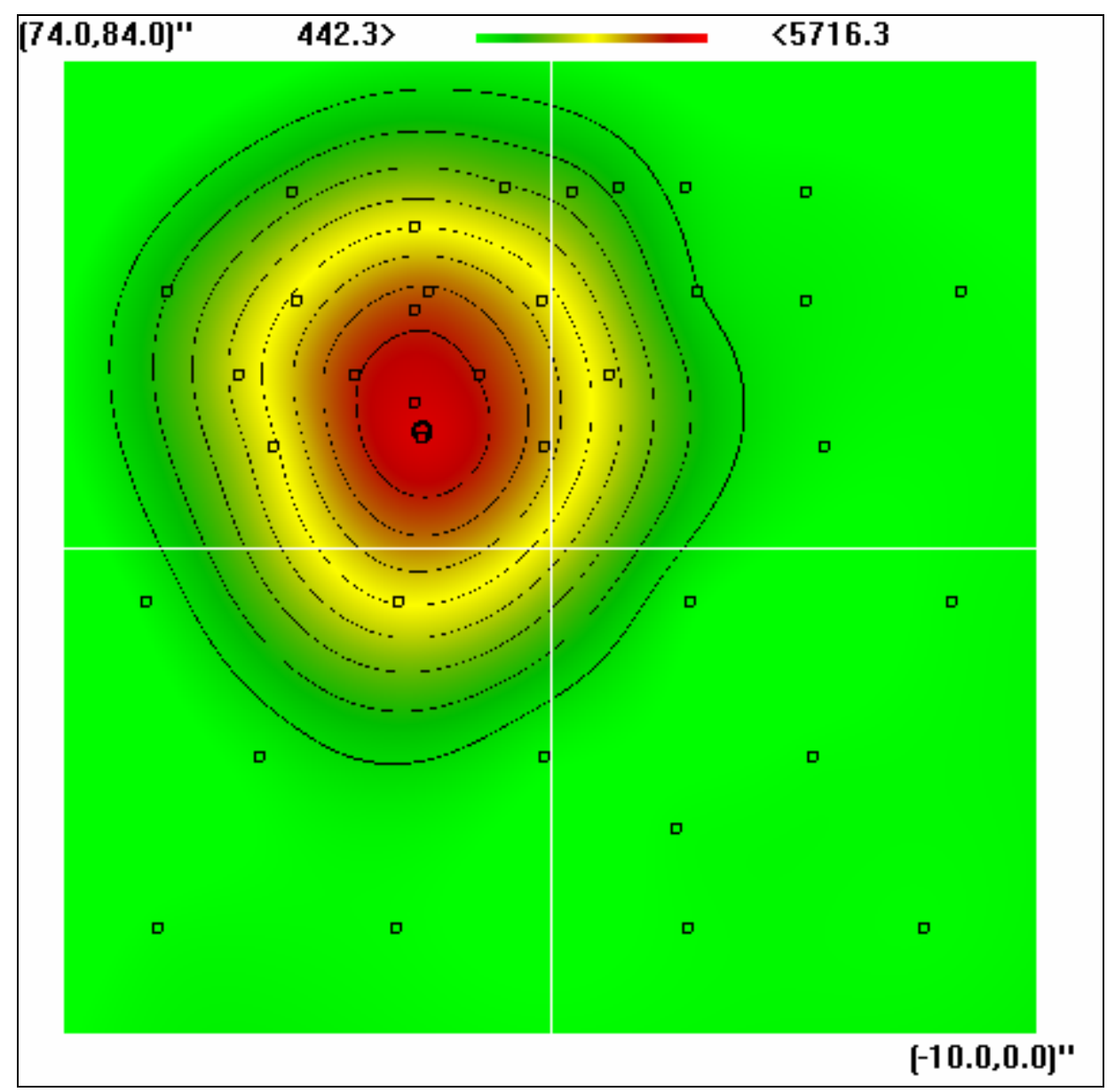

Figure 14 Example $\mathrm{CO}_{2}$ map (Color scale refers to ppm)

As can be seen in Figure 14, an infinite number of dilution ratios can be achieved within a given axial station. These span a finite value at the center of the plume to infinity at the plume's edge. Similar $\mathrm{CO}_{2}$ maps were acquired throughout the tunnel and used as a basis for the selection of aerosol size distribution sample locations. 
The squares within the map of Figure 14 represent actual sample point locations used to generate the map. The color scale at the top of the display represents the quantity being mapped, in this case $\mathrm{CO}_{2}$ concentration in (ppm). Black bands within the map represent division of this scale in increments of $10 \%$. The number pairs in the upper left and lower right corners of the display represent the upper left and lower right coordinates of the cross-sectional scan respectively (in inches). The reference for the horizontal coordinate of these number pairs was made arbitrarily from a common point located on the test apparatus, for convenience in calibration. Consistent horizontal references were maintained between stations by placing the center of the rig travel at the center of the tunnel for each cross-sectional scan. The reference for the vertical coordinate is always measured from the floor of each station.

Maps similar to those in Figure 14 were observed at other axial stations and were used as a basis for the selective placement of time consuming aerosol size distribution measurements. This aided overall collection efficiency and effectiveness, as a 3 point SMPS scan can account for more than twice as much time than that needed to produce a detailed $\mathrm{CO}_{2}$ map (as multiple SMPS samples per point were taken to obtain a representative average).

$\mathrm{CO}_{2}$ is typically used as a measure to determine plume expansion (i.e. dilution ratio). In laboratory dilution systems, this seems viable because of $\mathrm{CO}_{2}$ 's relative inertness and expansion is usually established within well mixed and confined environments. However for the study of unconstrained plumes, the concept of dilution may be a little less straight forward. 
The multitude of constituents present in diesel exhaust will naturally follow different rates of expansion, due to diffusive dissimilarities. Only a small number of these constituents such as $\mathrm{H}_{2} \mathrm{SO}_{4}$ represent a major contribution to the formation of diesel aerosols. In fact, due to its high vapor pressure, $\mathrm{CO}_{2}$ has little if anything to do with the creation of diesel aerosols. Therefore, using $\mathrm{CO}_{2}$ as a test metric of dilution, when nonuniform mixing of gases are observed, could lead to undesirably complex trends if any in the ordered data.

Nevertheless, being an initial study into the subject of precision exhaust mapping and to maintain consistency with prior emissions work, $\mathrm{CO}_{2}$ was used as a test metric in conjunction with dilution ratio with a cautious interpretation of its ability to describe plume dilution. This approach may ultimately be seen as to provide at least a qualitative description of plume expansion within the confines of each axial station.

There were many components associated with the collection of exhaust data at each axial station. All are tied into the test apparatus described in Section 1.4.3. Figure 15 describes the general layout of an axial station. 


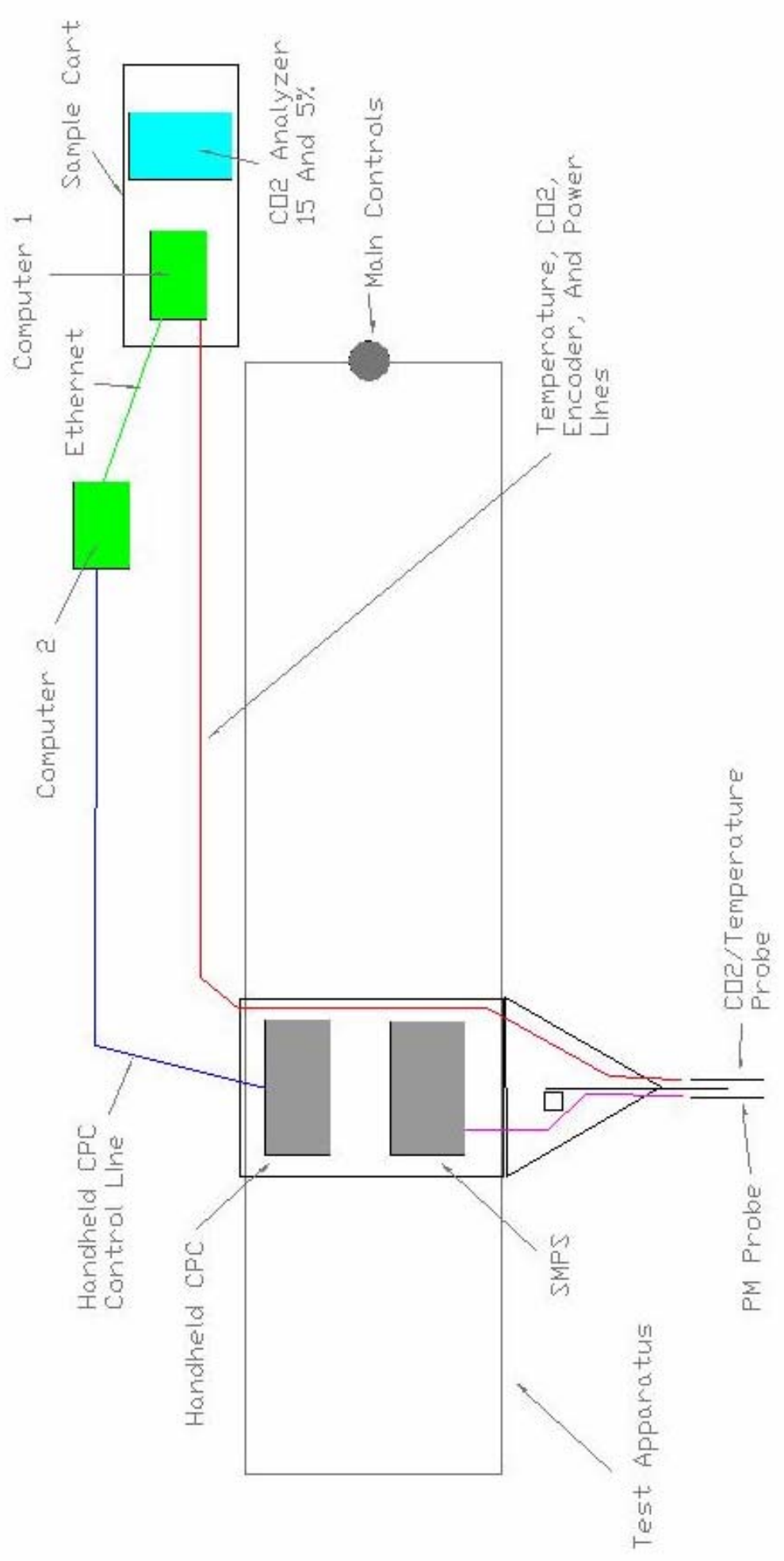

Figure 15 Axial Station Layout (Top View) 
As can be seen in Figure 15, a station scan included the measurements of $\mathrm{CO}_{2}(15 \%$ and 5\% channels of a California Analytical Model 300) which were blended in some cases, temperature, velocity, aerosol concentration (hand held CPC 3007), and aerosol size distribution (SMPS). Cross-sectional position was paired with all measurement quantities through the use of two encoders, geared to provide $\sim 0.1$ inch precision.

The sequence of operations for cross-sectional mapping once station set up was completed was the following.

- The main operator drove the probes to a given location (cross-sectional point and axial distance).

- Time was allocated to flood the sample lines with the gases from the newly positioned location $(\sim 15 \mathrm{sec})$.

- A sample was taken for a period which provided a reasonable average for the location ( 1min).

- The average values acquired for the current location were used to update the crosssectional scalar exhaust maps.

- The operator proceeded to collect data at a new point or was satisfied with the current map or maps and began SMPS measurements.

- The probes were driven to critical locations, dictated by the scalar exhaust maps, to collect aerosol size distribution data.

\subsubsection{Software}

Efficiency in the data collection process was benefited greatly by creating graphical software to be used during testing. This software was built about the visualization of the relative probe positions with respect to the plume. The visualization process began with 
the generation of two encoder signals representing the horizontal and vertical travel of the probes.

The encoder signals were routed directly to computer 1 shown in Figure 15 (by a PCMCIA card), which routinely performed a number of operations on them. The signals were first converted to a location array (based on encoder calibration at the beginning of the day) and sent to the screen. The array was then duplicated on the ether-network to computer 2 of Figure 15 for aerosol concentration processing. After that, the array was projected onto an image of the currently selected map $\left(\mathrm{CO}_{2}\right.$ or temperature) present on computer 1. Figure 16 provides an example of the probe projection and map display features for the same example of Figure 14.

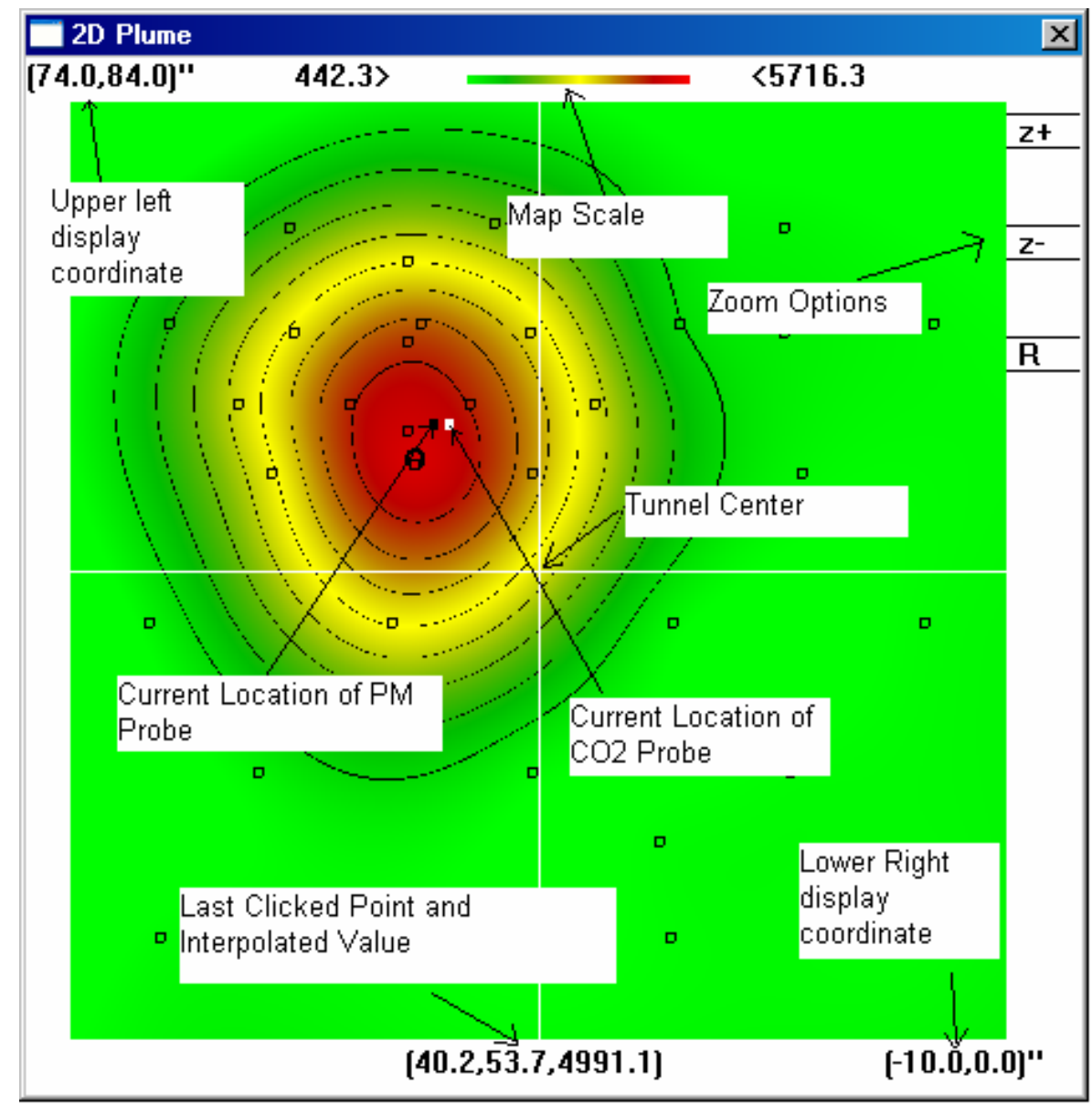

Figure 16 10ft Mapping Software Display Example 
The left black and right white squares of Figure 16 represent the current position of the aerosol and $\mathrm{CO}_{2}$ probes respectively, which have a 1.5 inch spacing. Temperature measurements were made directly between these two probes. As the operator drove the probes across the station plane, the position was continuously projected on the currently selected map. This approach allowed the operator to place the probes within areas of interest and / or low resolution for the current scan and benefited efficiency in scanning a plume of unknown form. During scans spanning small distances, the operator could also choose to zoom in with the options provided on the right side of the display screen. In addition, the operator could click on a point anywhere within the map to obtain the location and interpolated value of that point, which was displayed at the bottom of the screen. Clicking on the screen was also the basis for the center of the zoom option. The upper left and lower right number pairs represent the relative coordinates of the respective upper left and lower right corners of the display. The intersection of the white cross-hairs represents the center of the tunnel.

Data sets spaning more than three dimensions could also be viewed from the mapping software. By adding a projected depth to the data maps, two exhaust scalars could be viewed simultaneously throughout space. Figure 17 demonstrates a 4-dimensional exhaust map generated for the example of Figure 14. 


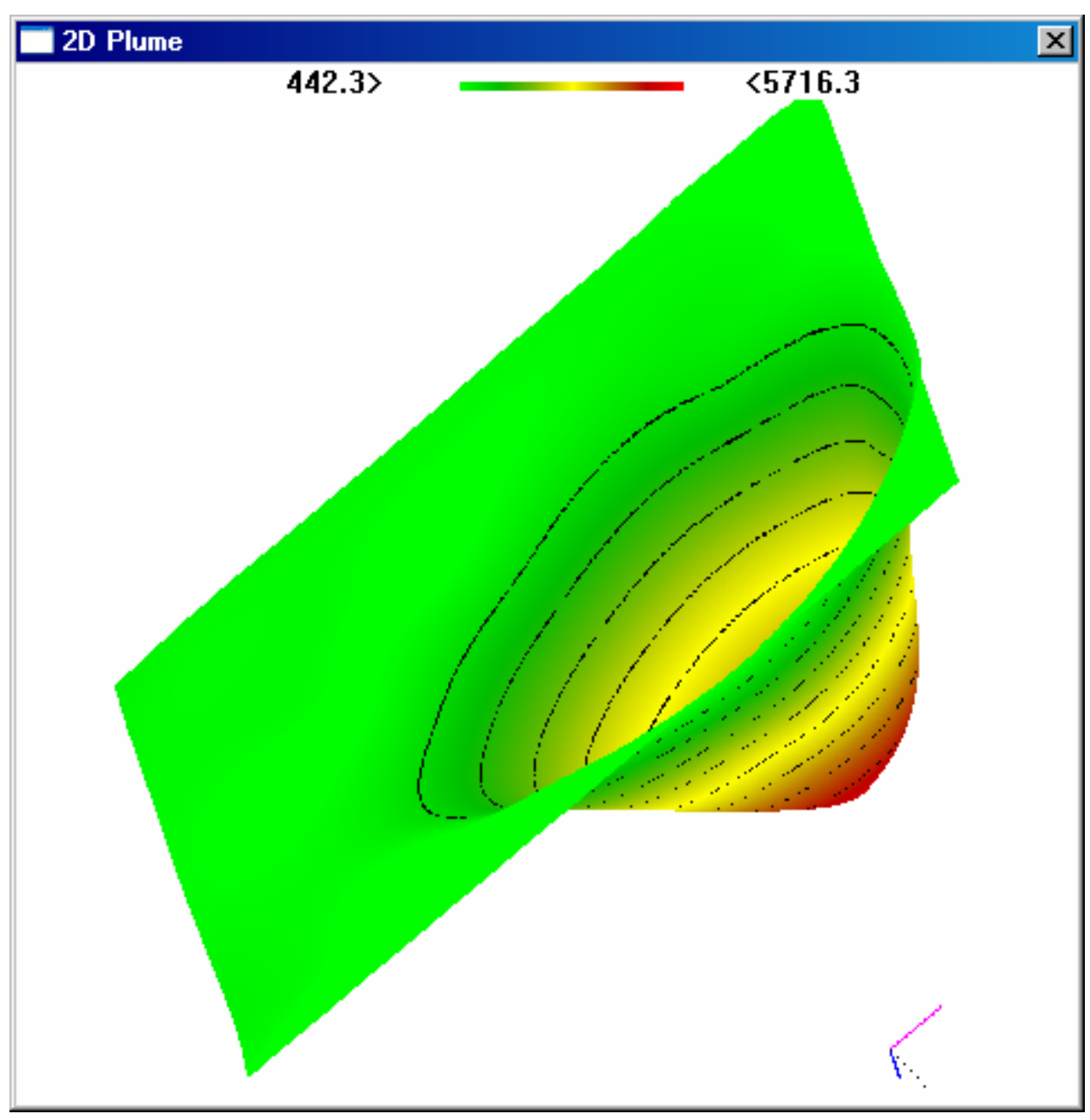

Figure 17 Example 4-Dimensional Exhaust Map of Figure 14

In this map color and depth are used to display the same quantities (CO2 concentration (ppm)). However, in the results section, similar maps will be used to show multiple exhaust scalars simultaneously. In addition, 3-dimensional surface contours of the data sets were made available through minor alterations to the software.

\subsection{Special Mapping Concerns}

\subsubsection{Scalar Maps}

The results of this study depended to a large extent on the ability to form meaningful exhaust averages within the tunnel. Under certain conditions, the averages formed within 
the tunnel may have no similarity to instantaneous measurements made of the plume. These dissimilarities can originate through a number of factors including massive plume oscillation due to turbulence, non-steady state effects and regular motion of the plume. Figure 18 describes what might happen to the similarity of the exhaust maps to instantaneous measurements of the plume, if the plume were to move in a regular fashion about a central point.

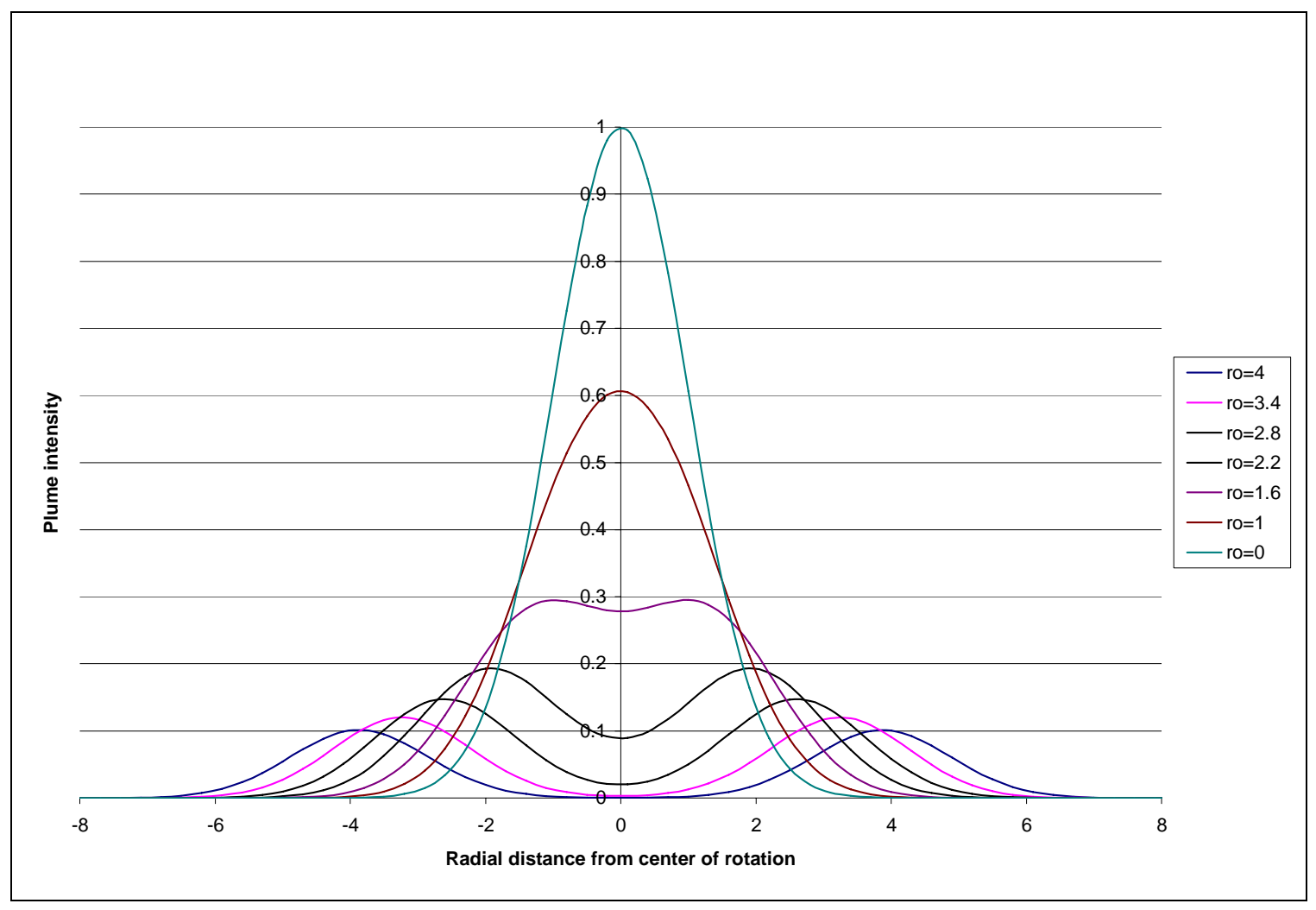

Figure 18 Qualitative Example of the Effect of Regular Plume Motion on Averaging.

As can be seen in Figure 18, dissimilarities between the scale of averaged distributions and instantaneous distributions (i.e. ro $=0$ ) grow with increasing travel of the plume. In addition, if this travel is regular, as in Figure 18, distortions in the shape of the plume may become evident as well. Irregular motions cause by turbulence will cause lowered peak concentrations to appear in the averages. 
Another issue that might arise when dealing with the results of this study is the validity of tracking a less time-expensive exhaust quantity to selectively sample a more time exhaustive one. This can become an issue if dissimilarities exist in the spatial characteristics of the two quantities. Being that aerosol size distribution forms physical connections to a multitude of scalar exhaust quantities, it might not be entirely feasible to use a single exhaust map in the anticipation of similar aerosol trends. This statement will be particularly true if dissimilarities exist within the scalar exhaust maps themselves.

Figure 19 describes a situation in which a third exhaust quantity depends on the sum of two other exhaust quantities with dissimilar expansion patterns.

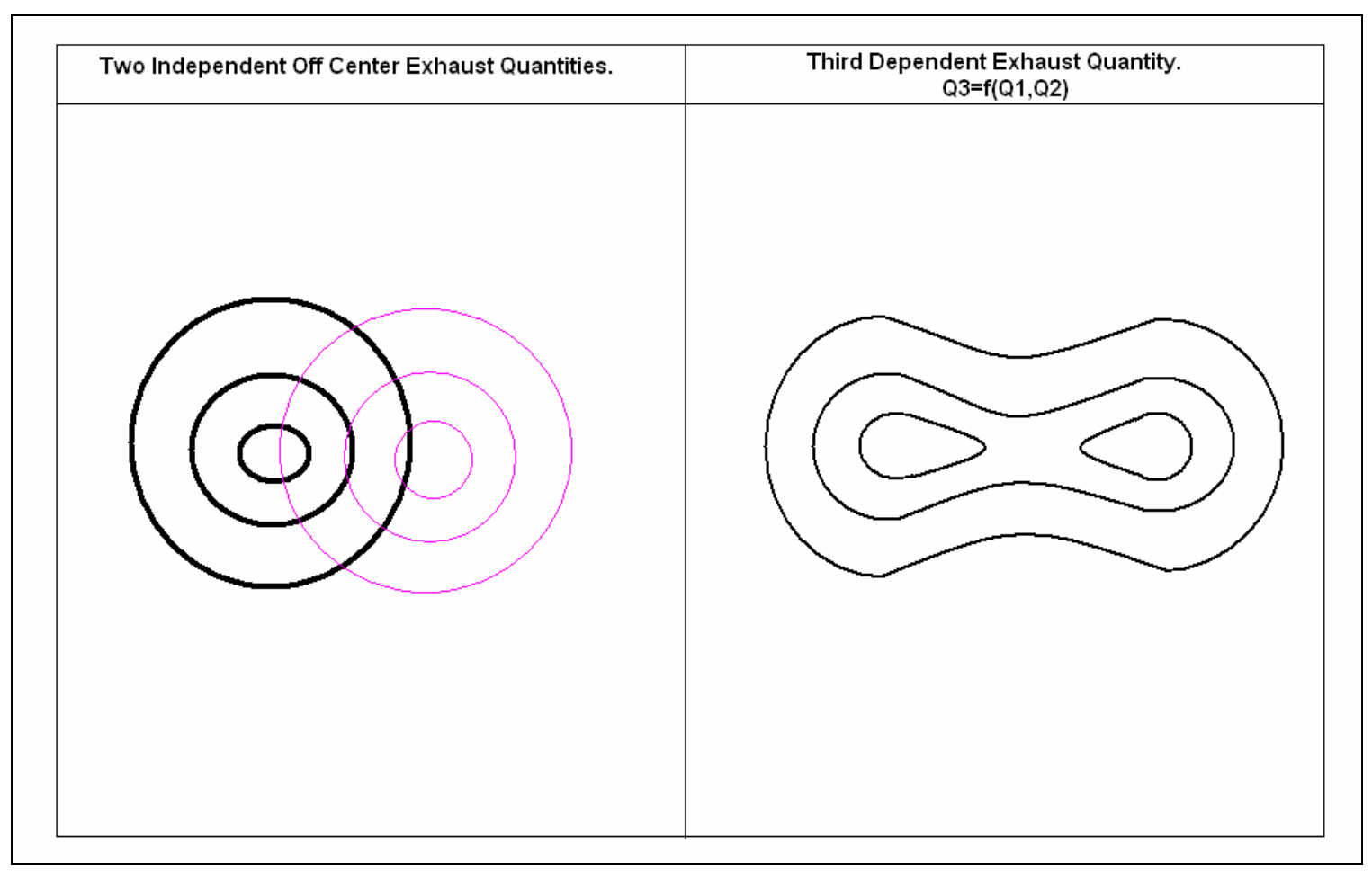

Figure 19 Illustrative Example of Coupled Map Dependencies.

As can be seen in Figure 19, tracking the third quantity by using any of the first two maps could result in unanticipated travel through diverse regions of the third map. This can 
introduce undesirably complex observations in the subsequent recorded trends of the third quantity. This issue will be discussed further in the results of Section 4 .

\subsubsection{Aerosol Sampling Losses}

Losses within the PM sampling lines can bias aerosol size distributions and reduce measurements of aersol concentration. Ayala et al. [30] has noted that "particle losses of more than 50\% are not uncommon and are not reported in the literature.” However, Ayala et al. [30] also noted that these losses are difficult to quantify due to theoretical limitations and require experimental measures.

Various mechanisms result in aerosol losses during sampling. These mechanisms include probe misalignment with respect to flow streamlines (that is, anisoaxial sampling [31]). Hangal and Willeke [32] has noted that anisoaxial sampling will result in impaction losses within the upper interior of the probe. During this study, the probes were aligned with the flow through the probe pivot shown in Figure 11 and anisoaxial sampling issues should be minimal.

Even when the probes are aligned with the flow (that is, isoaxial sampling [31]) as in this study, particle losses can originate from differences in the sample rate velocity compared to the flow velocity. However, Belyaev [33] showed that for low stokes numbers (i.e. $S t k \cong 10^{-2}$ ) differences in sampling velocity compared to flow velocity results in minimal sample loss. Kommer [34] showed that diesel exhaust aerosols have stokes numbers in the range of $2.4 \mathrm{e}^{-6}$ to $3.39 \mathrm{e}^{-2}$. Therefore, sampling rates should not have adversely compromised the sampling of aerosols within this study.

Aerosol samples can also be lost due to sample line transport through gravitational settling, diffusional, turbulent inertial, inertial at bends, flow constrictions, electrostatic, 
thermophoretic, and diffusiophoretic deposition [31]. Various studies have been performed to describe these processes [35, 36, 37, 38, 39, 40, 41, and 42], but each is specific and does not include coupled responses to multiple mechanisms. In this study, losses due to sample transfer were counteracted by using conductive rubber tubing and keeping the sample lines short (i.e. < 3ft), with smooth bends. In addition, similar studies [43] have shown minimal losses (i.e. -2 to $5 \%$ ) within similar aerosol transport systems.

\subsection{Plume Test Schedule}

Plume mapping was performed for a total of two engine settings (R75 and I30) over a 13 day test period comprised of 5 phases. Table 5 describes the phases of this study.

Table 5 Plume Test Schedule

\begin{tabular}{|c|c|c|}
\hline Phase & Test Days & Description \\
\hline 1 & $1,2,3,4$ & $\begin{array}{c}\text { Preliminary scalar exhaust mapping and } \\
\text { system setup. Engine @ R75 }\end{array}$ \\
\hline 2 & $5,6,7$ & $\begin{array}{c}\text { Thorough scalar exhaust mapping with } \\
\text { aerosol size distribution measurements. } \\
\text { Engine @ R75 }\end{array}$ \\
\hline 3 & $8,9,10$ & $\begin{array}{c}\text { Thorough scalar exhaust mapping with } \\
\text { aerosol size distribution measurements. } \\
\text { Engine @ I30 }\end{array}$ \\
\hline 4 & 11 & $\begin{array}{c}\text { Mixed flow test with two house fans } \\
\text { installed in tunnel. Engine @ R75 }\end{array}$ \\
\hline 5 & 12,13 & $\begin{array}{c}\text { Fine aerosol size distribution resolution } \\
\text { (1 to 10ft). Engine @ R75 }\end{array}$ \\
\hline
\end{tabular}

Throughout the remainder of this document, results will be referred to by the day they were taken. As such, a given day will be used to describe background conditions, engine setting and raw exhaust quantities to be provided in the results section. 


\subsection{Dimensional Analysis}

Several factors make this study unique to the environment it was performed in. A list of some of the more important influences is provided in Table 6.

Table 6 Summary of Dimensional Characteristics of Plume Study

\begin{tabular}{|c|c|}
\hline Dimension & Description \\
\hline$U_{\text {Vent }}$ & Ventilation air velocity \\
\hline$T_{\text {Vent }}$ & Ventilation air temperature \\
\hline$R H_{\text {Vent }}$ & Ventilation air relative humidity \\
\hline$\rho_{\text {Vent }}$ & Ventilation air density \\
\hline$\alpha_{\text {Vent }}$ & Ventilation air thermal diffusivity \\
\hline$L_{\text {Tunnel }}$ & Tunnel length \\
\hline$A_{\text {Tunnel }}$ & Tunnel cross-sectional area \\
\hline$H_{\text {Tunnel }}$ & Tunnel height \\
\hline$\frac{d H}{d L}_{\text {Tunnel }}$ & Tunnel slope \\
\hline $\operatorname{Diam}_{\text {Exh }}$ & Exhaust diameter \\
\hline$\alpha_{E x h}$ & Exhaust air thermal diffusivity \\
\hline$\rho_{E x h}$ & Exhaust air density \\
\hline$T_{E x h}$ & Exhaust air temperature \\
\hline$D_{\mathrm{CO}_{2}}$ & Exhaust $\mathrm{CO}_{2}$ diffusivity \\
\hline$U_{E x h}$ & Exhaust air velocity \\
\hline
\end{tabular}


Through non-dimensional analysis, with the assumed primary influence of the variables found in Table 6, the following non-dimensional parameters will dictate similarity to other experiments.

- $\frac{U_{\text {Vent }}^{2} A_{\text {Tunnel }}}{D_{\mathrm{CO}_{2}}^{2}}$

- $\frac{U_{\text {Exh }}^{2} A_{\text {Tunnel }}}{D_{\mathrm{CO}_{2}}^{2}}$

- $\frac{\alpha_{\text {Vent }}}{D_{\mathrm{CO}_{2}}}$

- $\frac{\rho_{\text {Vent }}}{\rho_{\text {Exh }}}$

- $\frac{L_{\text {Tunnel }}^{2}}{A_{\text {Tunnel }}}$

- $\frac{H_{\text {Tunnel }}^{2}}{A_{\text {Tunnel }}}$

- $\frac{T_{\text {Vent }}}{T_{E x h}}$

- $\frac{d H}{d L}_{\text {Tunnel }}$

- $\frac{\text { Diam }_{\text {Exh }}^{2}}{A_{\text {Tunnel }}}$

- $\frac{\alpha_{E x h}}{D_{\mathrm{CO}_{2}}}$

- $R H_{\text {Vent }}$ 
However, these variables do not account for the highly specific features of this study. Results specific to this sudy may be influenced by the specific engine being used, fuel, blockage arrangements within the tunnel, engine set point, and waviness in the walls of the tunnel. Due to the uniqueness and complexity of these influences, similarity in the lab and / or other experimental environments may be difficult to achieve by matching the above mentioned non-dimensional variables.

In addition, the Reynolds number ( $\mathrm{Re}$ ) should also be matched to achieve similarity within the tunnel. Where Re is given by [22]

$$
\operatorname{Re}=\frac{U D_{H}}{v}
$$

Equation 43

Where $\mathrm{U}$ is the velocity of the tunnel air ( $\sim 40 \mathrm{ft} / \mathrm{min}), v\left(\sim 15.09 e^{-6} \mathrm{~m}^{2} / \mathrm{s} \quad[23]\right)$ is the kinematic viscosity of the tunnel air, and $D_{H}(\sim 10.2 \mathrm{ft})$ is the hydraulic diameter of the tunnel given by [24].

$$
D_{H}=4 \frac{\text { Area }_{\text {Cross-section }}}{\text { Perimeter }_{\text {Cross-section }}}
$$

This results in a tunnel Reynolds number of 41380 . Under this Reynolds number, flow should be turbulent [25] (i.e. Re>10000). In addition, local variations in tunnel geometry can further induce turbulence [26]. Therefore, measurements made near the walls of the tunnel can have large turbulent intensities. However, as noted in [27 and 28] free stream turbulence has little effect on the sampling efficiency of aerosols. Turbulence will however, increase the deposition rate within the sampling lines as noted in [29], which will alter aerosol measurements. 


\section{Results}

The results of this study were divided into four main sections. The first (Background and Raw Exhaust Data) will list all relevant background and raw exhaust measurements made and to be used implicitly throughout the remainder of the text. The next section (Scalar Plume Development) will establish the nature of the plume as a whole, during its scalar development within the tunnel test region. The third section (Aerosol Formation and Development) will discuss the aerosol size distribution trends, as they were selectively acquired from the plume. Lastly, a section will be devoted towards uncertainty in the methods and devices used within the study.

\subsection{Background and Raw Exhaust Data}

The engine was operated over two steady-state modes of operation during this study. These were chosen as R75 and I30 to demonstrate two extremes of engine operation. Figure 20 and Figure 21 provide the raw exhaust $\mathrm{CO}_{2}$ and temperature data respectively, taken under R75 and I30 operation. 


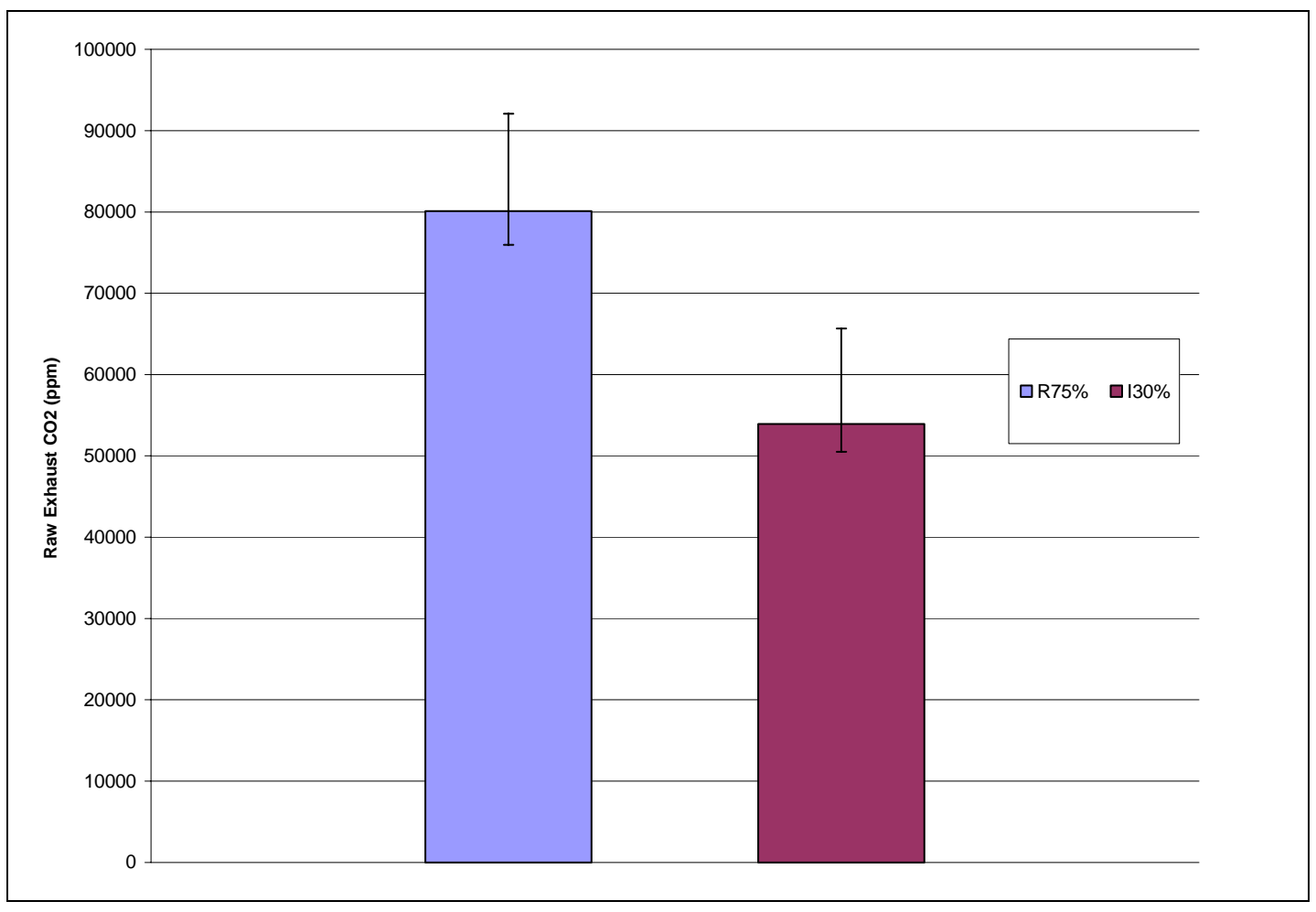

Figure 20 Raw Exhaust $\mathrm{CO}_{2}$ Data Taken Under R75 and I30 Operation.

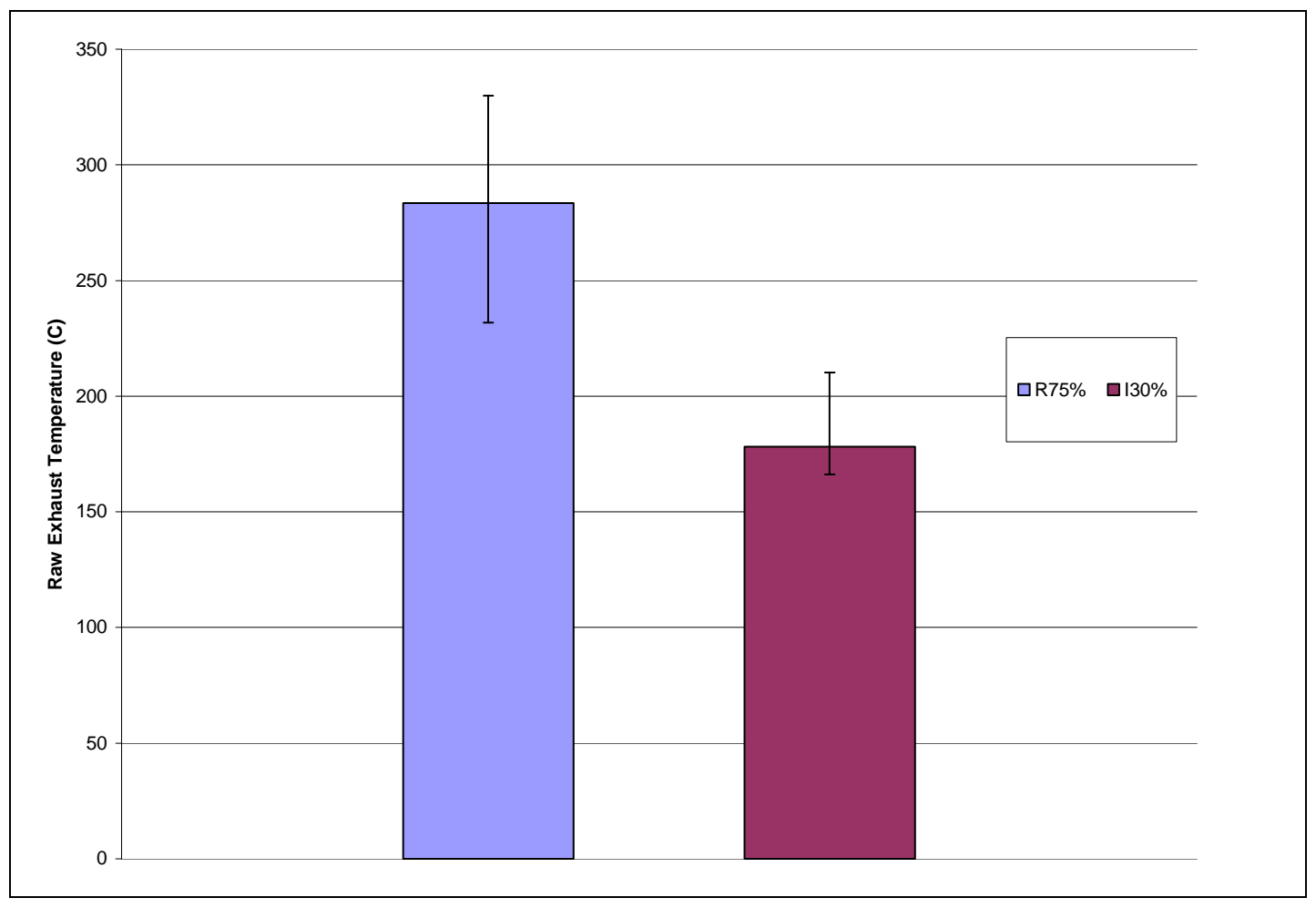

Figure 21 Raw Exhaust Temperature Data Taken Under R75 and I30 Operation. (Thermocouple located 2 feet ahead of exhaust outlet) 
The data from Figure 20 results in an average raw exhaust $\mathrm{CO}_{2}$ concentration of 80,000 ppm and 55,000 ppm for R75 and I30 operation respectively. These raw exhaust concentrations will be used throughout the remainder of this study, whenever an engine setting is cited. Figure 21 results in an average raw exhaust temperature of $275 \mathrm{C}$ and 175 C for R75 and I30 operation respectively. These raw exhaust temperatures will also be used throughout the remainder of this study, whenever an engine setting is cited.

In addition to the collected raw exhaust data, various background quantities were recorded daily. Table 7 provides a summary of the test conditions observed for each of the 13 test dates.

Table 7 Summary of Recorded Background and Raw Exhaust Measurements

\begin{tabular}{|c|c|c|c|c|c|c|c|c|}
\hline Day & Date & $\begin{array}{c}\text { Engine } \\
\text { Mode }\end{array}$ & $\begin{array}{c}\text { Raw } \\
\mathrm{CO}_{2} \\
(\mathrm{ppm})\end{array}$ & $\begin{array}{c}\text { Raw } \\
\text { Exhaust } \\
\text { Temp. } \\
(\mathrm{C})\end{array}$ & $\begin{array}{c}\text { Setpoint } \\
\text { Tunnel } \\
\text { Flow } \\
\text { (cfm) }\end{array}$ & $\begin{array}{c}\text { Average } \\
\text { Background } \\
\text { Temp. (F) }\end{array}$ & $\begin{array}{c}\text { Average } \\
\text { Background } \\
\mathrm{CO}_{2}(\mathrm{ppm})\end{array}$ & $\begin{array}{c}\text { Relative } \\
\text { Humidity } \\
(\%)\end{array}$ \\
\hline 1 & $9 / 14 / 06$ & $\mathrm{R} 75$ & 80000 & 275 & 10000 & 57 & 560 & 61 \\
\hline 2 & $9 / 19 / 06$ & R75 & 80000 & 275 & 10000 & 58 & 500 & 61 \\
\hline 3 & $10 / 3 / 06$ & R75 & 80000 & 275 & 10000 & 57 & 520 & 75 \\
\hline 4 & $10 / 5 / 06$ & R75 & 80000 & 275 & 10000 & 57 & 480 & 98 \\
\hline 5 & $10 / 17 / 06$ & R75 & 80000 & 275 & 10000 & 51 & 452 & 72 \\
\hline 6 & $10 / 24 / 06$ & R75 & 80000 & 275 & 10000 & 52 & 438 & 25 \\
\hline 7 & $10 / 26 / 06$ & R75 & 80000 & 275 & 10000 & 56 & 508 & 12 \\
\hline 8 & $10 / 31 / 06$ & I30 & 55000 & 175 & 10000 & 55 & 411 & 22 \\
\hline 9 & $11 / 6 / 06$ & I30 & 55000 & 175 & 10000 & 52 & 555 & 11 \\
\hline 10 & $11 / 8 / 06$ & I30 & 55000 & 175 & 10000 & 54 & 426 & 63 \\
\hline 11 & $11 / 14 / 06$ & R75 & 80000 & 275 & 10000 & 56 & 597 & 30 \\
\hline 12 & $11 / 28 / 06$ & R75 & 80000 & 275 & 10000 & 54 & 620 & 14 \\
\hline 13 & $11 / 30 / 06$ & R75 & 80000 & 275 & 10000 & 56 & 512 & 69 \\
\hline
\end{tabular}

Throughout the remainder of this study, test results will be described in terms of the day they were taken, as this will implicitly provide all background and engine operation conditions. 


\subsection{Scalar Plume Development}

The following sections describe the scalar mapping results of this study. Primary emphasis is placed on the results obtained during days 5 through 10 described in Table 7. These maps will be used to justify the chosen aerosol sample placement locations within the plume and to validate the completeness found in the subsequent aerosol evolutionary trends.

\subsection{1 $\mathrm{CO}_{2}$ I Dilution}

This section describes the $\mathrm{CO}_{2}$ mapping results of the study. These maps will ultimately be used with raw exhaust and background $\mathrm{CO}_{2}$ measurements to calculate exhaust dilution ratio (DR) throughout the tunnel. Dilution ratio will later be validated and used as a test metric for the selectively placed aerosol size distribution data. This section will be divided into two parts representing the different engine settings used during the study. Maps will be listed in sequential order of axial placement to provide an indication of plume development. In addition, some maps will be shown more than once to demonstrate day to day repeatability.

\subsubsection{R75}

Figure 22 through Figure 31 provide the $\mathrm{CO}_{2}$ mapping results taken on days 5 through 7 with the engine set at R75. Figure 22 begins this sequence at an axial placement of 11 inches. 


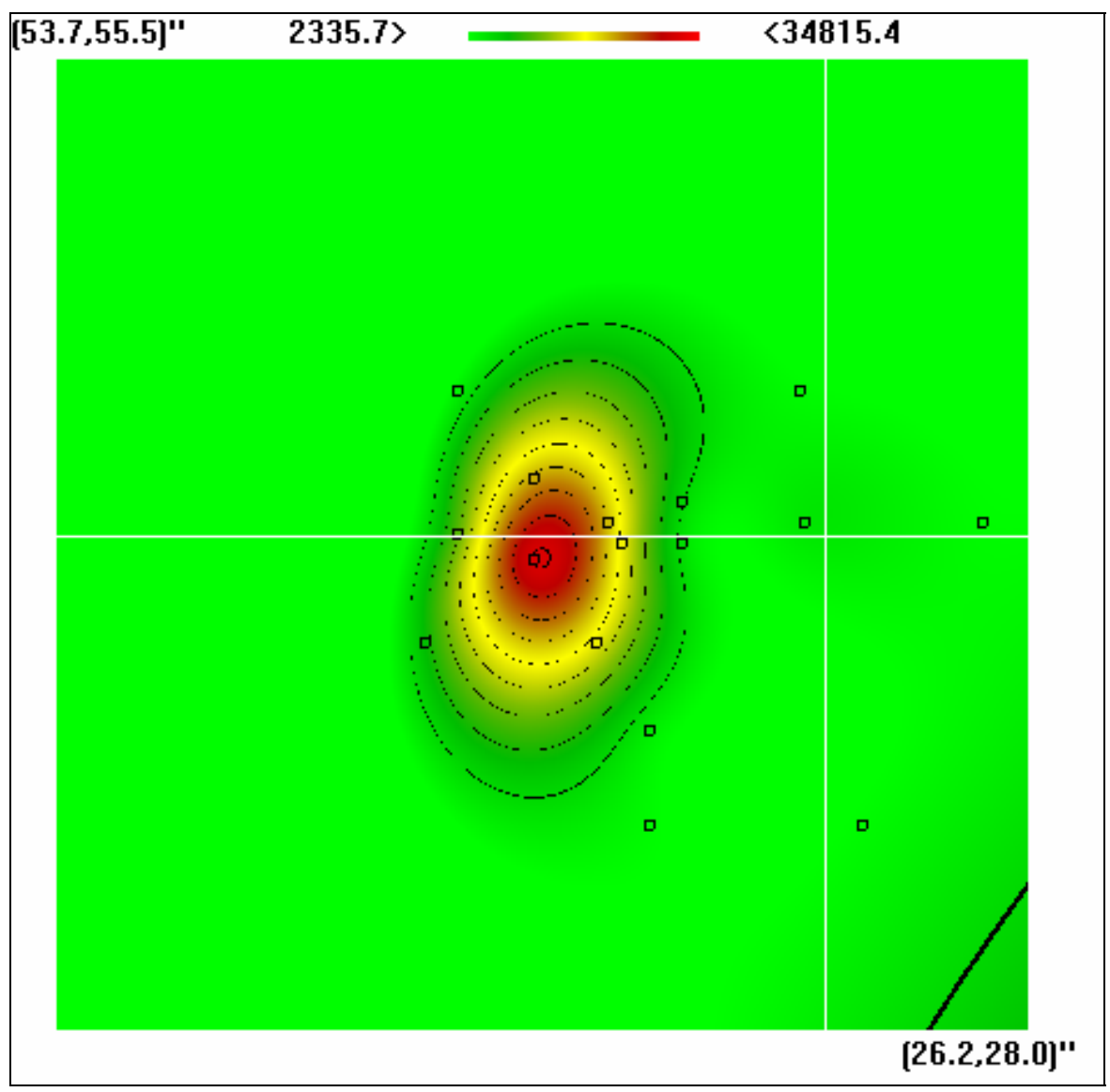

Figure $22 \mathrm{CO}_{2}$ Mapping Results for an Axial Placement of 11in (Taken on Day 7). (Color Scale Refers to CO2 concentration (ppm))

Figure 22 indicates that the plume has expanded to a diameter, based on $\mathrm{CO}_{2}$, of roughly 8inches at an axial distance of 11inches (as mentioned in Section 3 coordinate pairs represent horizontal and vertical distances in inches unless otherwise noted). This size is roughly three times the raw exhaust outlet dimension (i.e. 3 inch diameter exhaust pipe). In addition, the expansion has occurred at a non-uniform rate resulting in an infinite range of DRs emanating from a finite center. This center has been reduced by more than half of its raw $\mathrm{CO}_{2}$ concentration of $80,000 \mathrm{ppm}$.

The map of Figure 22 was acquired through the use of analyzer channel 2 (15\% max) as the $\mathrm{CO}_{2}$ levels read at this station would likely peak out channel 1 (5\% max) in the 
inner regions of the plume. As such the outer measurement regions of the plume cannot be considered dependable as channel 2 offers little precision for measurements made near background levels. If dilution quantities from the outer plume regions are required, they will be calculated from measurements simultaneously stored for channel 1 . In addition, this map was created prior to the addition of the zooming feature of the mapping software. As such, sample placement suffers in efficiency, as a number of points were acquired outside any major plume influence.

The next $\mathrm{CO}_{2}$ mapping for this engine setting was recorded at an axial placement of $6 \mathrm{ft}$. This map was repeated to demonstrate day to day consistency. Figure 23 and Figure 24 provide the $\mathrm{CO}_{2}$ maps taken for days 5 and 6 respectively.

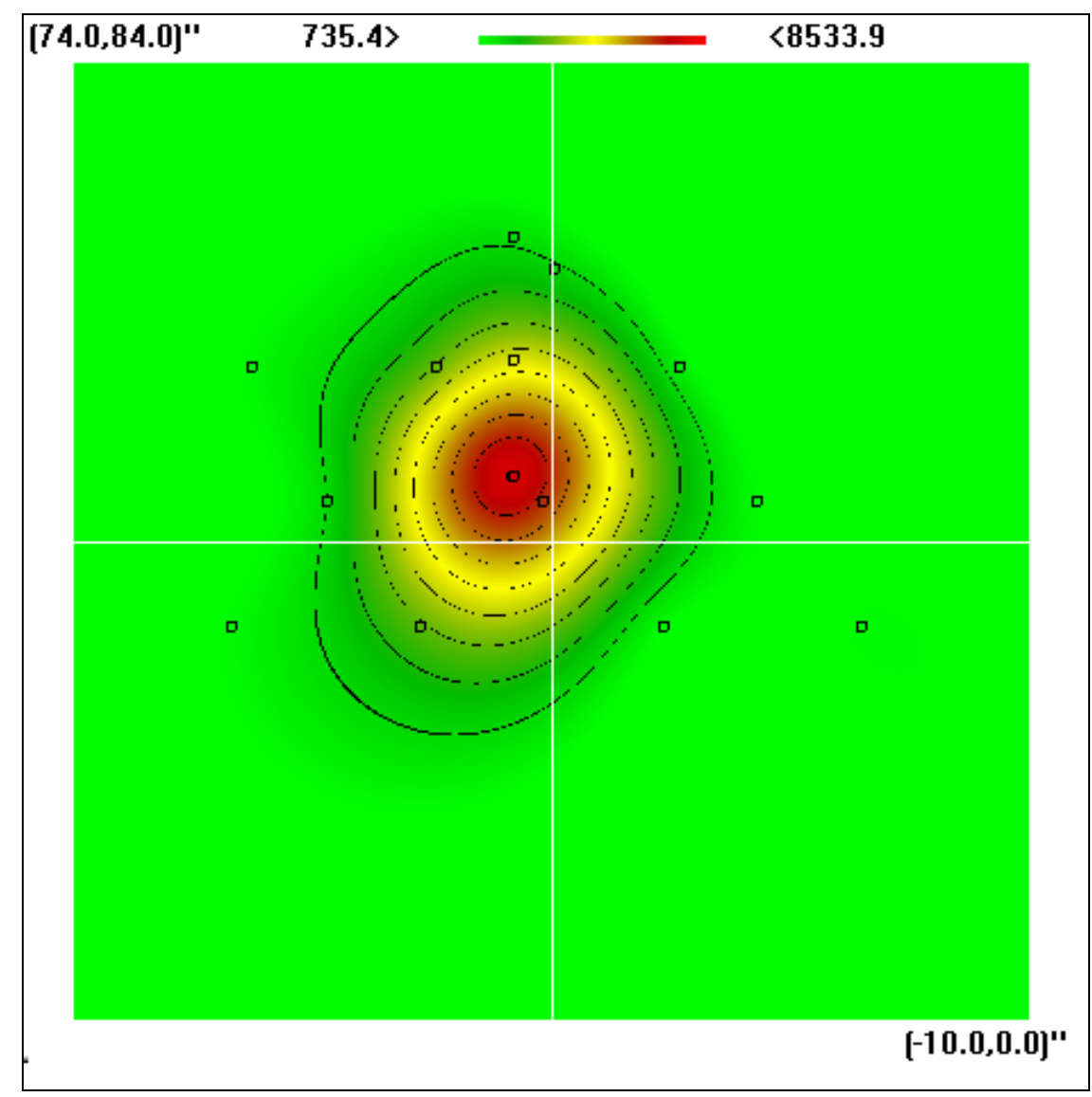

Figure $23 \mathrm{CO}_{2}$ Mapping Results for an Axial Placement of 6ft (Taken on Day 5). (Color Scale Refers to CO2 concentration (ppm)) 


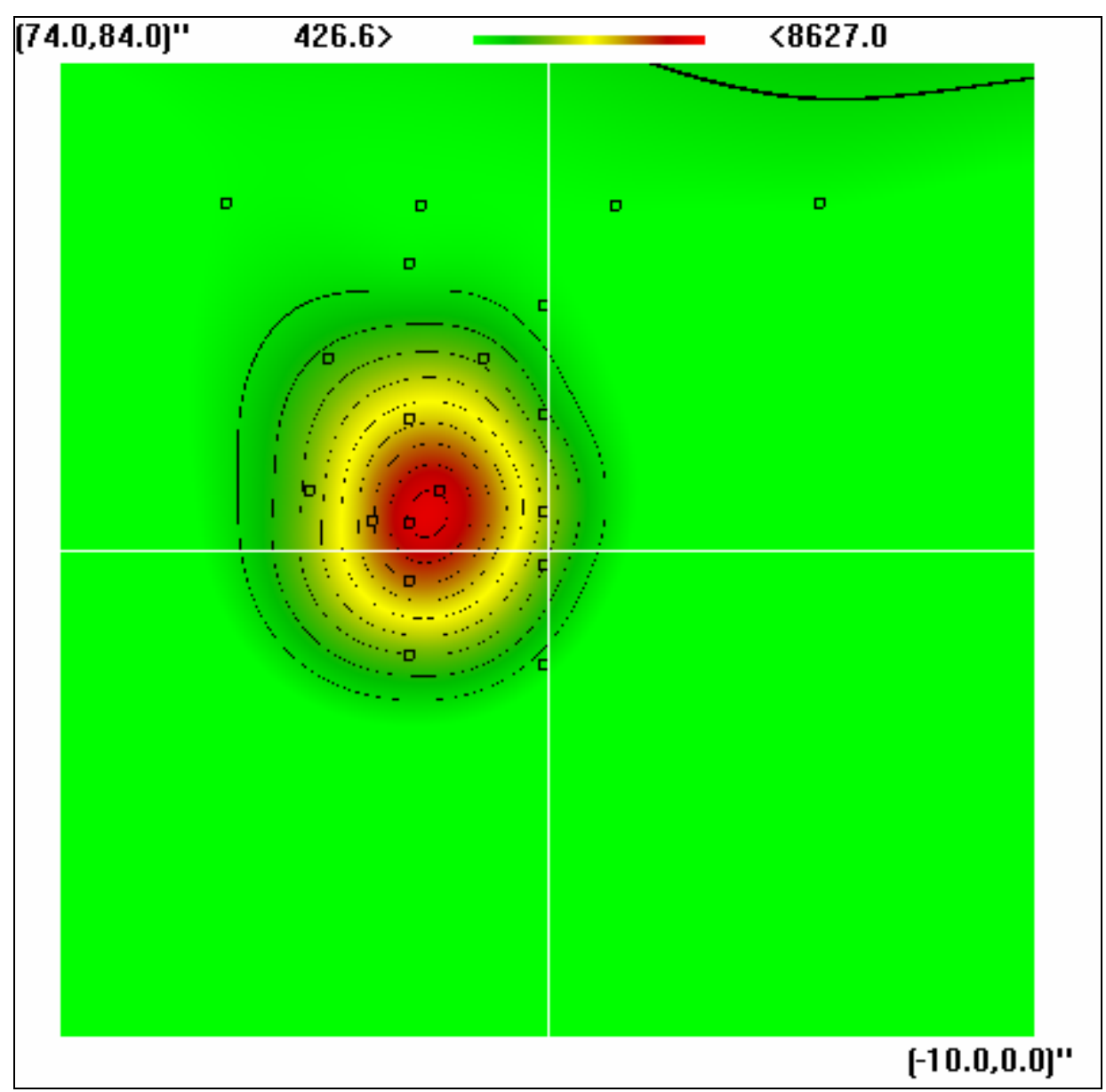

Figure $24 \mathrm{CO}_{2}$ Mapping Results for an Axial Placement of 6ft (Taken on Day 6). (Color Scale Refers to CO2 concentration (ppm))

As can be seen, in both Figure 23 and Figure 24 the center of the plume has risen slightly from the plume center found at the 11inch station. This is likely caused by the buoyant effects of the hot exhaust as it travels through the relatively cool tunnel. In addition, the relative size of the plume (roughly 35 inch diameter) at $6 \mathrm{ft}$ has grown by a factor of more than 10 from the exhaust outlet. This size increase leads to peak $\mathrm{CO}_{2}$ measurements that are roughly one $10^{\text {th }}$ of their raw exhaust equivalents.

Figure 23 and Figure 24 also indicate that the plume has moved slightly to the left during the day 6 mapping as compared to the day 5 mapping. This can be the result of many factors including the placement of foreign objects within the tunnel and or a 
gradual misalignment of the exhaust pipe. Nevertheless, the overall size and shape of the plume has changed little between the two days. In addition, peak $\mathrm{CO}_{2}$ levels appear consistent producing a relative error of close to $1 \%$. Background variation is a little less favorable and could be caused by the inadvertent opening of the tunnel door while work was being performed in the mine. This can cause an unrecorded increase in tunnel flow, as the venturi only accounts for the flow that travels through it and not the door. It was also found that if the door was not locked properly, it would oscillate between being open and closed producing irregular variations in measurements made upstream. This was resolved during the coarse of this study by the installation of a more robust steel frame door.

The next $\mathrm{CO}_{2}$ mapping made under R75 operation was recorded at an axial placement of $10 \mathrm{ft}$. This map is given in Figure 25. 


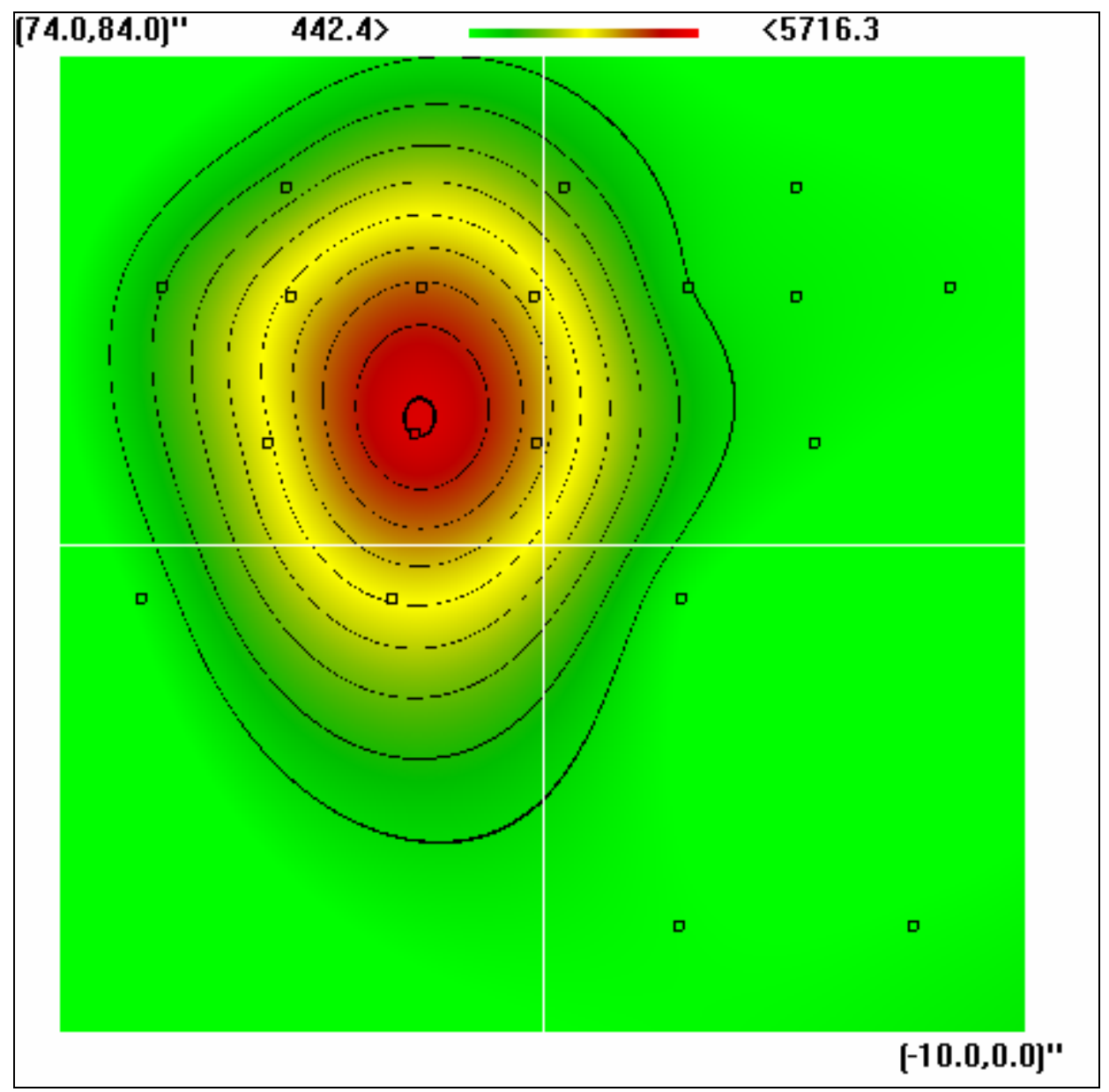

Figure $25 \mathrm{CO}_{2}$ Mapping Results for an Axial Placement of 10ft (Taken on Day 7).

(Color Scale Refers to CO2 concentration (ppm))

The plume has grown appreciably at this station but still maintains a concentric form.

Peak $\mathrm{CO}_{2}$ concentrations have fallen by nearly $3000 \mathrm{ppm}$ from the $6 \mathrm{ft}$ axial station. The plume has also become slightly elongated (vertically), perhaps due to its continual rise. This can also be an indication of oscillations found in the plume's vertical position, which can bleed into the shape formed from averaging. This explanation appears more viable, as any significant rise should be seen as a compression in the upper contours of the plume due to a higher buoyant potential of the plume's center. In fact, the tendency of the plume towards the tunnel ceiling may be more influenced by the slope present in the tunnel than buoyancy. 
The effect of the rising and expanding plume has resulted in the top of the plume making contact with the tunnel ceiling at the $10 \mathrm{ft}$ station. In addition, although peak levels of $\mathrm{CO}_{2}$ have fallen drastically from exhaust measurements, spatial variation in $\mathrm{CO}_{2}$ still exceeds $1300 \%$ at the $10 \mathrm{ft}$ station, which is easily detected by the lower channel of the instrumentation.

The next $\mathrm{CO}_{2}$ mappings were recorded at an axial distance of 20 feet. These maps were repeated to demonstrate day to day consistency. Figure 26 and Figure 27 illustrate the $\mathrm{CO}_{2}$ maps taken for this axial placement.

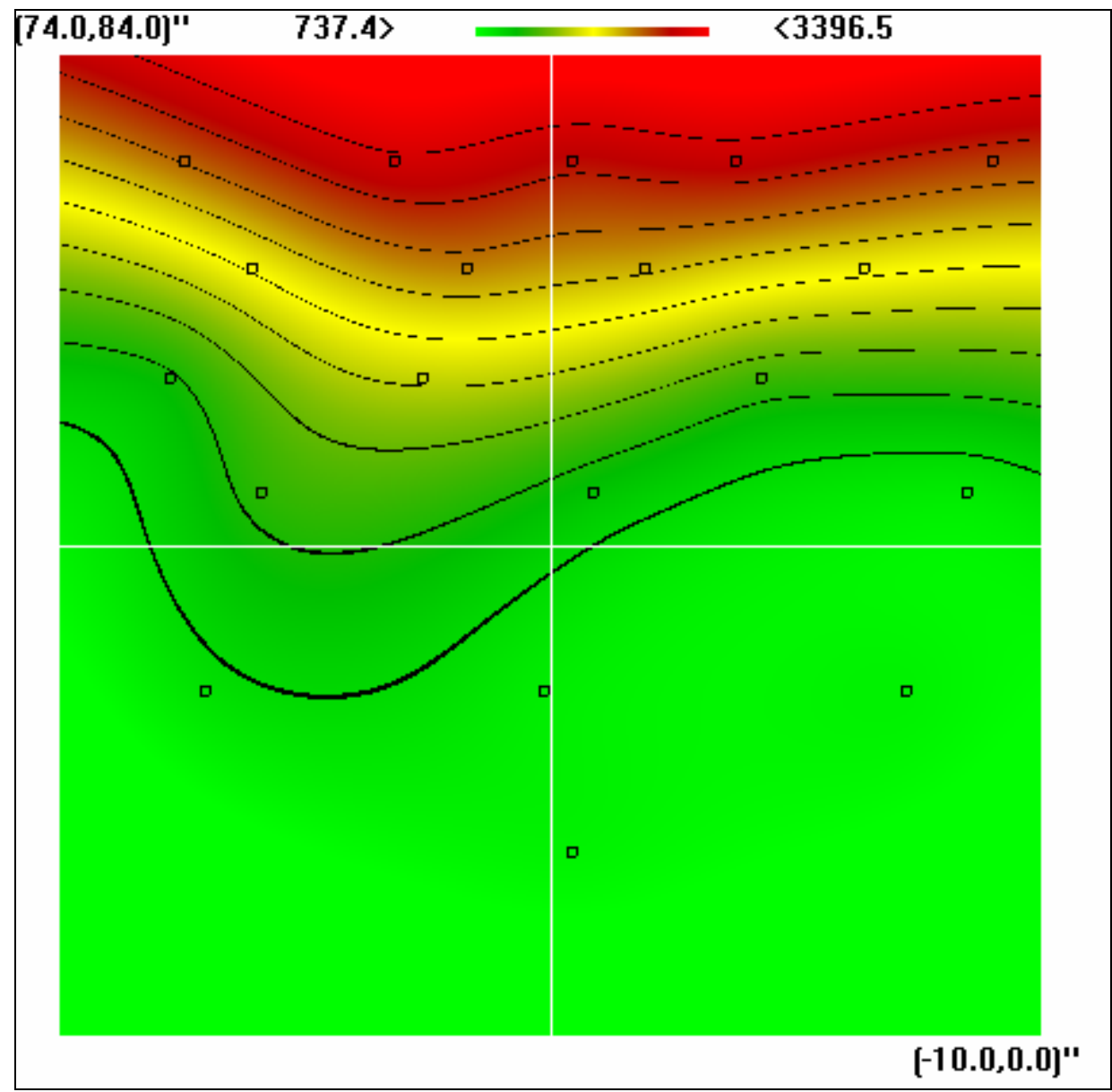

Figure $26 \mathrm{CO}_{2}$ Mapping Results for an Axial Placement of 20ft (Taken on Day 5).

(Color Scale Refers to CO2 concentration (ppm)) 


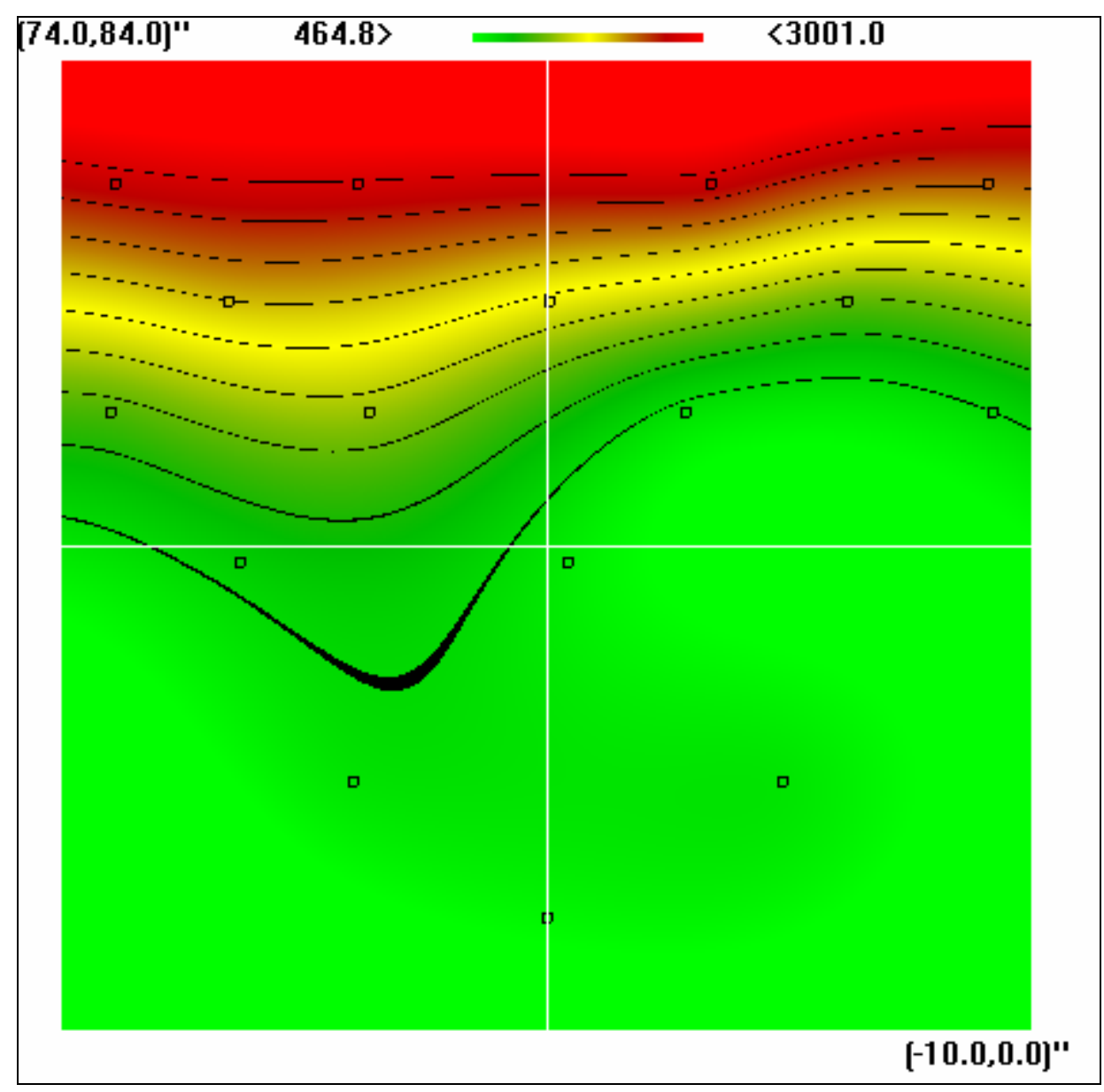

Figure $27 \mathrm{CO}_{2}$ Mapping Results for an Axial Placement of 20ft (Taken on Day 6). (Color Scale Refers to CO2 concentration (ppm))

As can be seen in Figure 26 and Figure 27, the plume has made full contact with the tunnel ceiling at 20 feet and stratification of $\mathrm{CO}_{2}$ is apparent. A slight warping of the contour lines towards the left of the tunnel (observed in both days) suggests that the plume has not fully risen at this station. In addition, peak $\mathrm{CO}_{2}$ measurements have dropped by 2500 ppm from the 10 foot station. As such, it is not expected that the plume spans the full width of the tunnel at 20 feet, in spite of the fact that it has reached the limits of the test aparatus's horizontal travel.

Day to day variation at 20 feet is generally agreeable producing a relative error of $\sim 12 \%$ in peak recorded $\mathrm{CO}_{2}$ measurements. However, day to day variation in 
background $\mathrm{CO}_{2}$ measurements is still relatively large. Nevertheless, background $\mathrm{CO}_{2}$ measurements appear to be consistent throughout a single day’s scan. As can be seen in the maps of Figure 24 and Figure 27 taken on day 6, outer plume $\mathrm{CO}_{2}$ measurements are nearly identical between the 6 and 20 foot stations. The same is also true for the mapping results taken on day 5 .

The next $\mathrm{CO}_{2}$ mapping of the study was recorded on day 7 at an axial placement of 50ft. This map is shown in Figure 28.

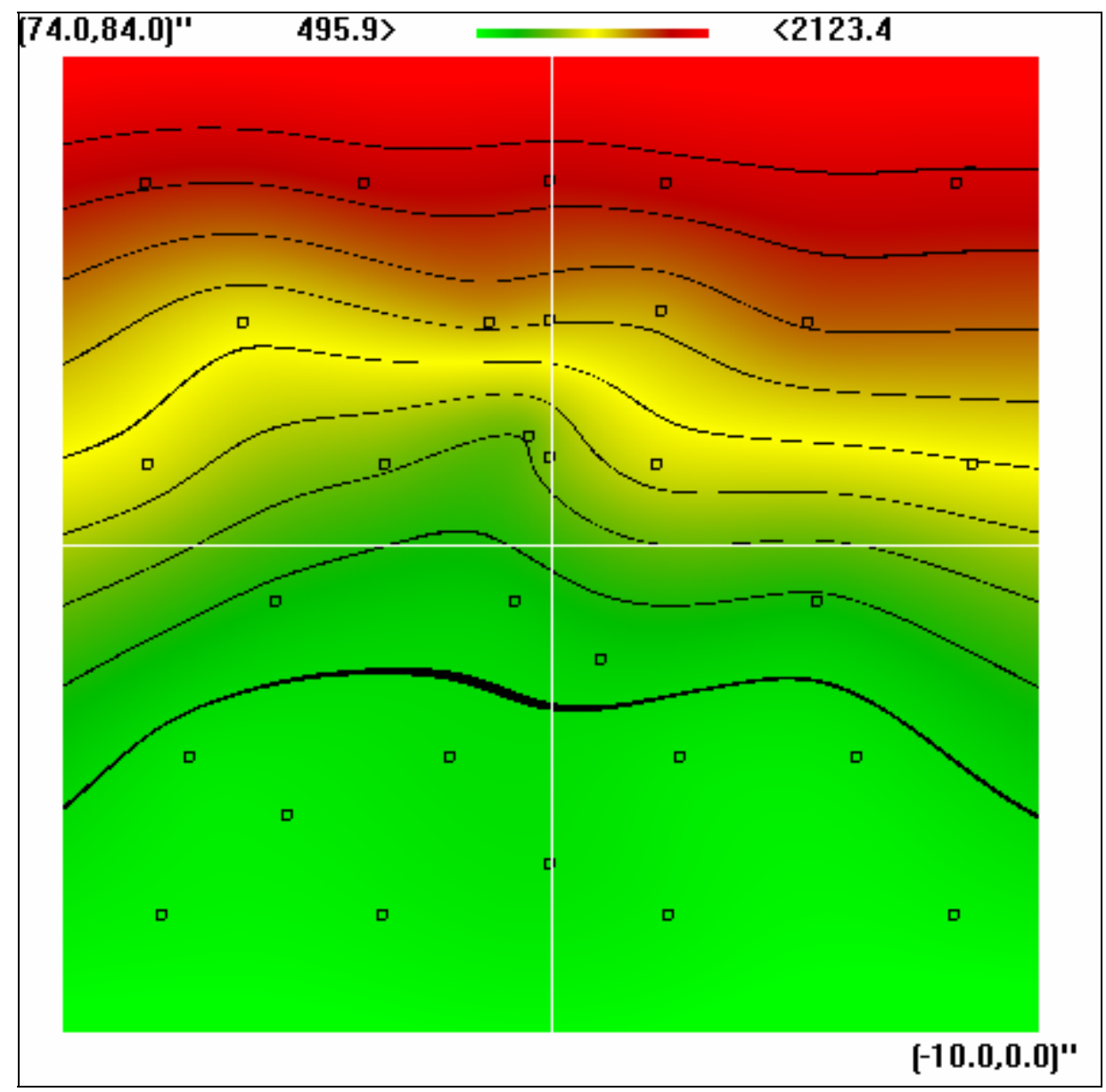

Figure $28 \mathrm{CO}_{2}$ Mapping Results for an Axial Placement of 50ft (Taken on Day 7). (Color Scale Refers to CO2 concentration (ppm)) 
As can be seen in the map of Figure 28, stratification has continued from the 20 to 50 foot stations. In addition, peak $\mathrm{CO}_{2}$ measurements have dropped by roughly $900 \mathrm{ppm}$ for the 30 foot span between these two stations. This small drop further suggests that the plume found at the 20 foot station does not fully span the width of the tunnel. Even at a relatively distant placement of 50 feet, spatial variation in peak and minimum recorded levels of $\mathrm{CO}_{2}$ still exceed $400 \%$.

The waviness observed in the center of the map found in Figure 28 is thought to be the result of a permanently fixed hanging obstruction, used to hold instrumentation, located roughly 40 feet from the exhaust outlet. This obstruction is shown in Figure 29.

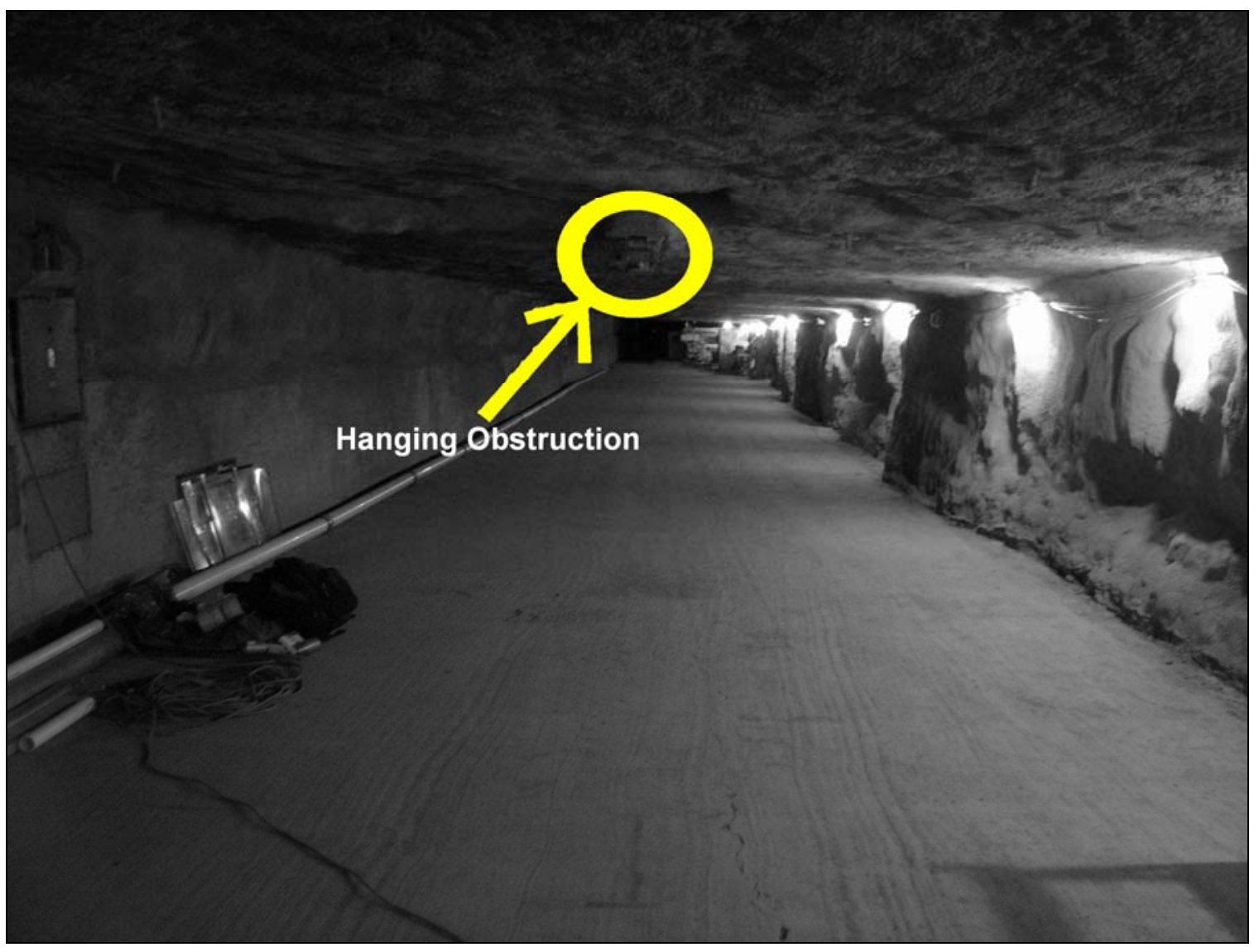

Figure 29 Hanging Obstruction Found Ahead of the 50ft Axial Station 
In addition to this continual disruption, scans were occasionally disturbed by ongoing construction within the tunnel, which required personel to walk through the test region. An example of such a disturbance will be discussed for the next station located at 125 feet. These variations can typically be drawn out of the data averages through added sample time. However, if they persistently occur during a test they can determine a new average all together. This makes understanding plume behavior, based on averaging, a less viable and more difficult task.

The next $\mathrm{CO}_{2}$ mapping was performed at an axial placement of 125 feet. This map was repeated to demonstrate day to day consistency. Figure 30 and Figure 31 illustrate the maps of the 125 foot station, taken on days 5 and 6 respectively. 


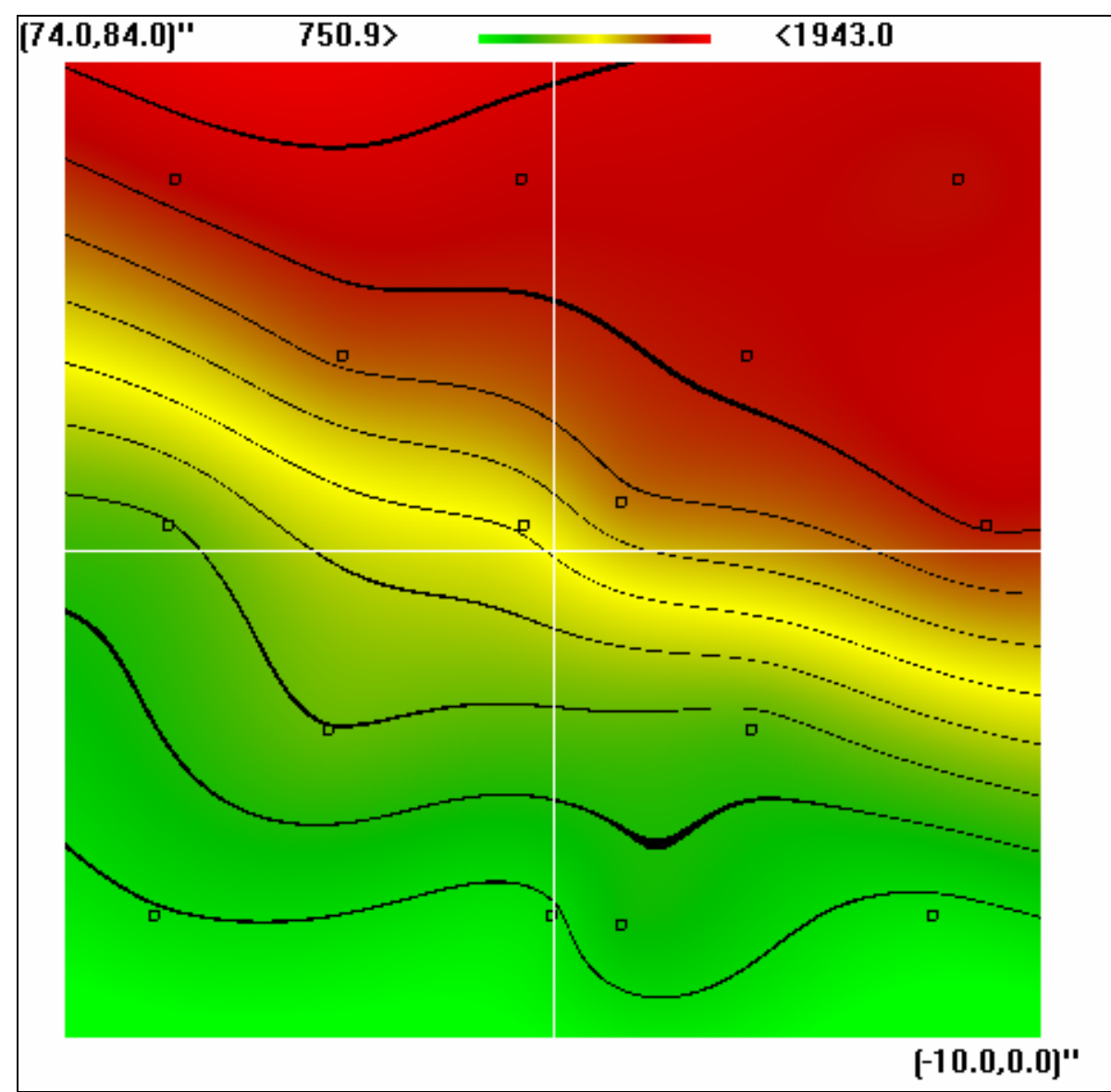

Figure $30 \mathrm{CO}_{2}$ Mapping Results for an Axial Placement of $125 f t$ (Taken on Day 5). (Color Scale Refers to CO2 concentration (ppm)) 


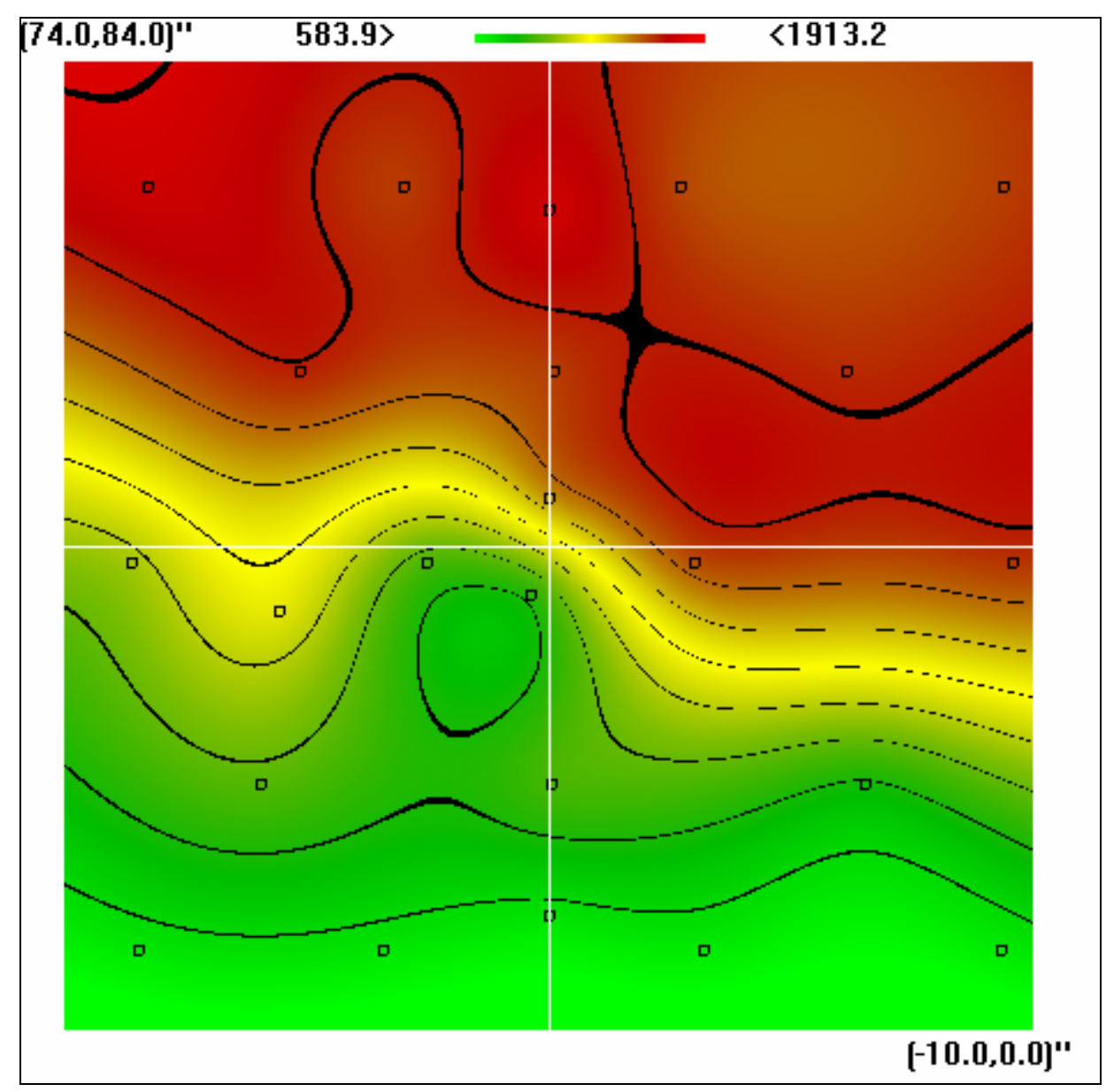

Figure $31 \mathrm{CO}_{2}$ Mapping Results for an Axial Placement of 125ft (Taken on Day 6).

(Color Scale Refers to CO2 concentration (ppm))

Inspection of the maps found in Figure 30 and Figure 31 reveal that day to day variation in peak $\mathrm{CO}_{2}$ measurements are small for the 125 foot station, producing a relative error of only $\sim 1.5 \%$. There are notable differences, however, in the form of the plume between these two days (mainly the waviness recorded on day 6). The waviness in the day 6 map is due to worker interference in the tunnel. During this scan, workers repeatedly walked up and down the center of the tunnel. In spite of this, sampling continued due to time limitations and to demonstrate the potential effect of such disturbances. Even though the plume could not be maintained in a consistent 
environment during the scan of day 6, general plume behavior is still observable by the map (i.e. stratification).

In both maps of Figure 30 and Figure 31, there is a slight slant of the $\mathrm{CO}_{2}$ contours towards the lower right of the tunnel. This slant is most likely due to the steep slope of the tunnel ceiling in the same direction of the contours at this location. In addition, leveling of the test rig may also exaggerate this slope as this can cause a rotation of the cross-sectional coordinate system with respect to the flow. This can be alleviated in future studies through a coupled calibration of the encoder inputs.

Due to the high similarity of the contours (in number and shape) at the 125 and 50 foot stations, the remainder of the tunnel was not mapped for R75 operation to allow time for mapping to be made at a different engine setting.

\subsubsection{I30}

Figure 32 through Figure 41 describe the $\mathrm{CO}_{2}$ mapping results as they were taken axially on days 8 through 9 with the engine set at I30. Maps were not repeated for any station due to time constraints. Figure 32 provides the $\mathrm{CO}_{2}$ mapping results taken at an axial distance of 11 inches. 


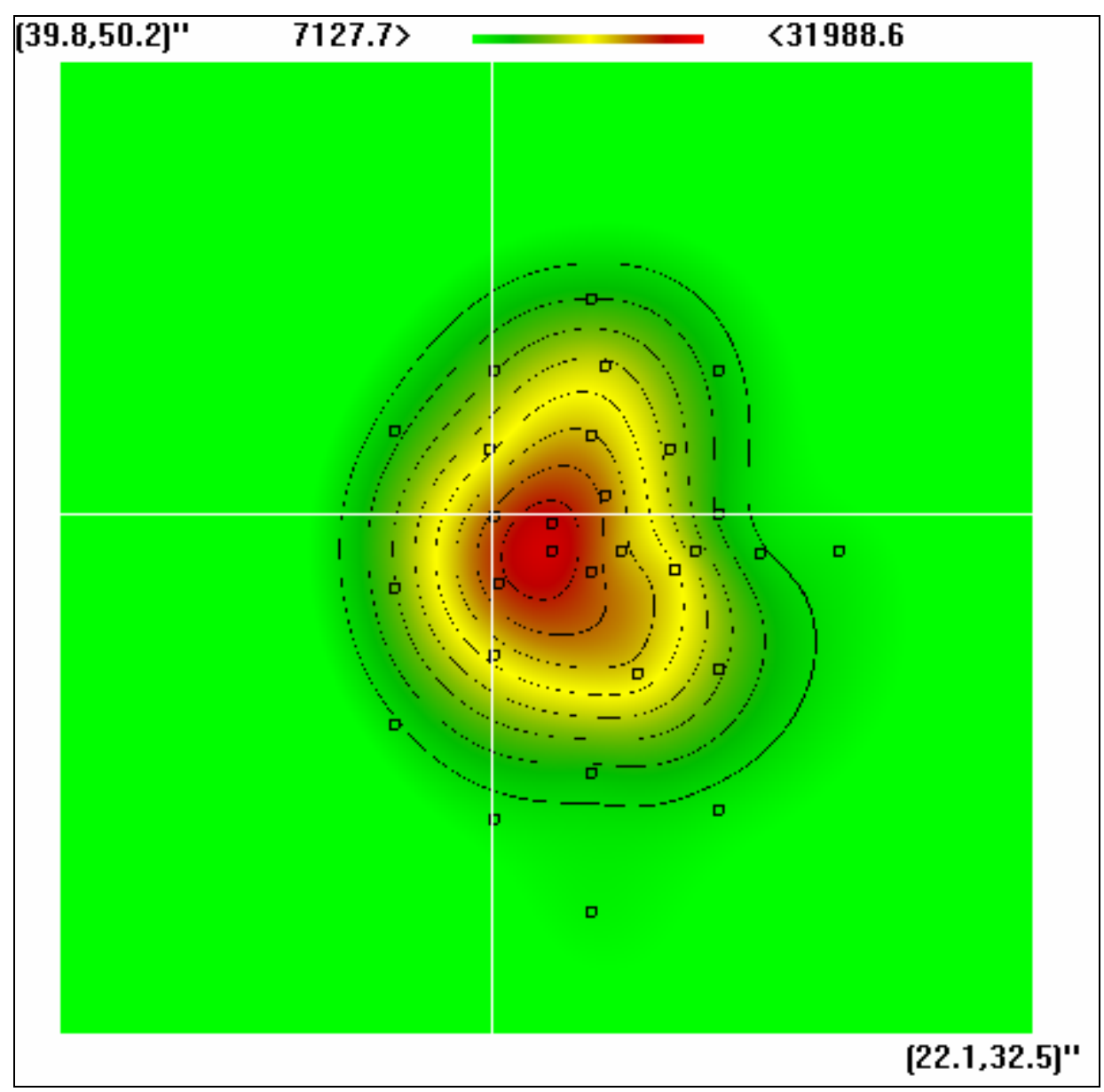

Figure $32 \mathrm{CO}_{2}$ Mapping Results for an Axial Placement of 11in (Taken on Day 10).

(Color Scale Refers to CO2 concentration (ppm))

The map of Figure 32 provides a little more resolution than its R75 counterpart of

Figure 22, due to the advent of the zooming feature described in Section 2.2.1. As in

Figure 22, the generation of this map was established by the collection of channel 2 data.

When outer plume dilution ratios were required to order the aerosol size distribution data, channel 1 data was used.

Figure 33 shows the $\mathrm{CO}_{2}$ mapping results made an axial placement of $6 \mathrm{ft}$. 


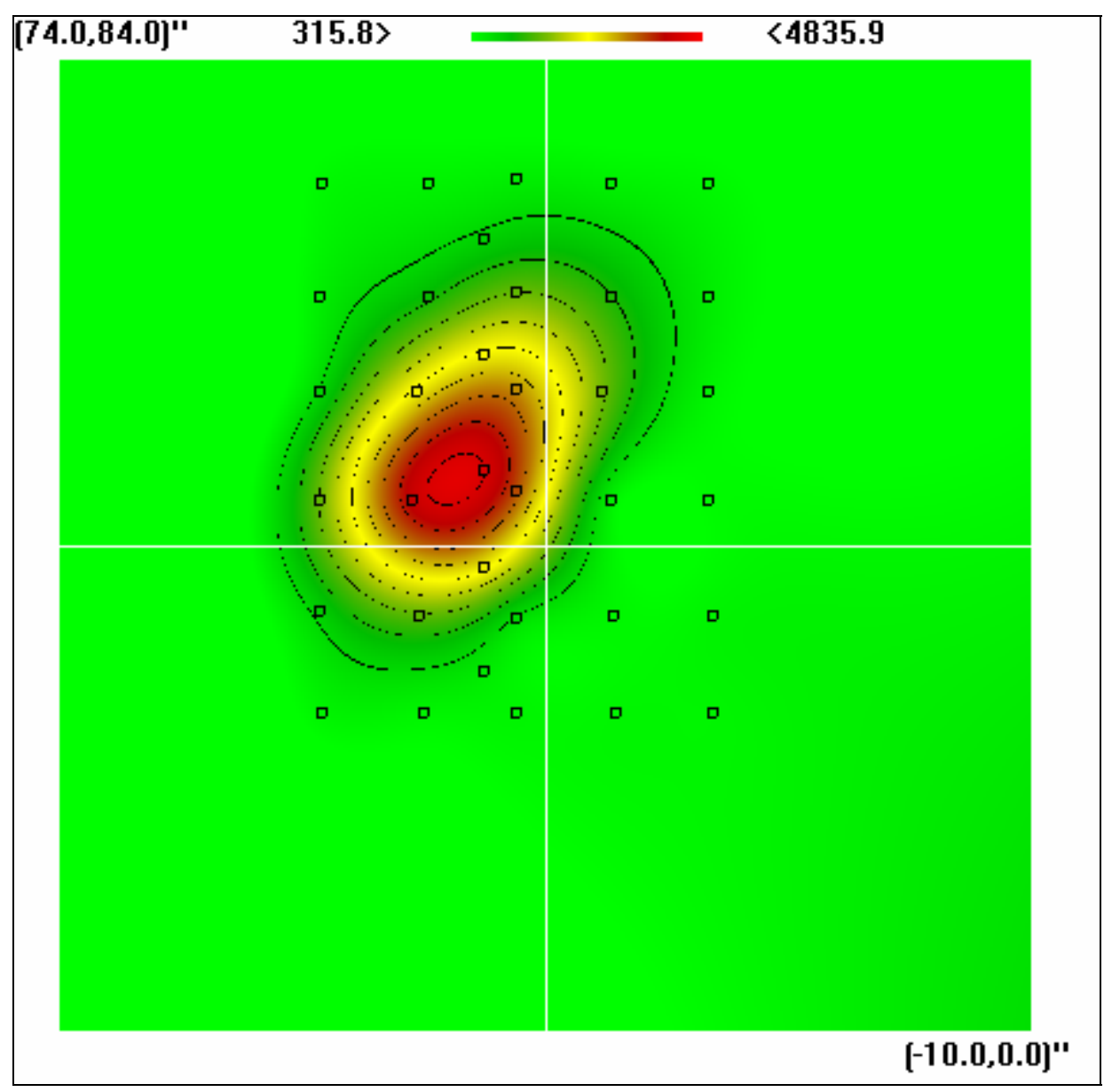

Figure $33 \mathrm{CO}_{2}$ Mapping Results for an Axial Placement of 6ft (Taken on Day 8).

(Color Scale Refers to CO2 concentration (ppm))

Other than differences in the $\mathrm{CO}_{2}$ concentrations, the shape and size of this map is nearly identical to the maps made at the same station for R75 operation. Since repeated station scans were not to be made during this phase, more time could be spent on finer resolution of the test plane. This map was created through a more uniform grid pattern and demonstrates that a "blind" scan of the plume can still result in a detailed $\mathrm{CO}_{2}$ map. In addition, the generation of this contour was made with nearly 3 times as many sample points as that made at the 6ft station for day 5 and does not show any appreciable resolution benefit in the map.

Figure 34 shows the $\mathrm{CO}_{2}$ mapping made at the $10 \mathrm{ft}$ station on day 8. 


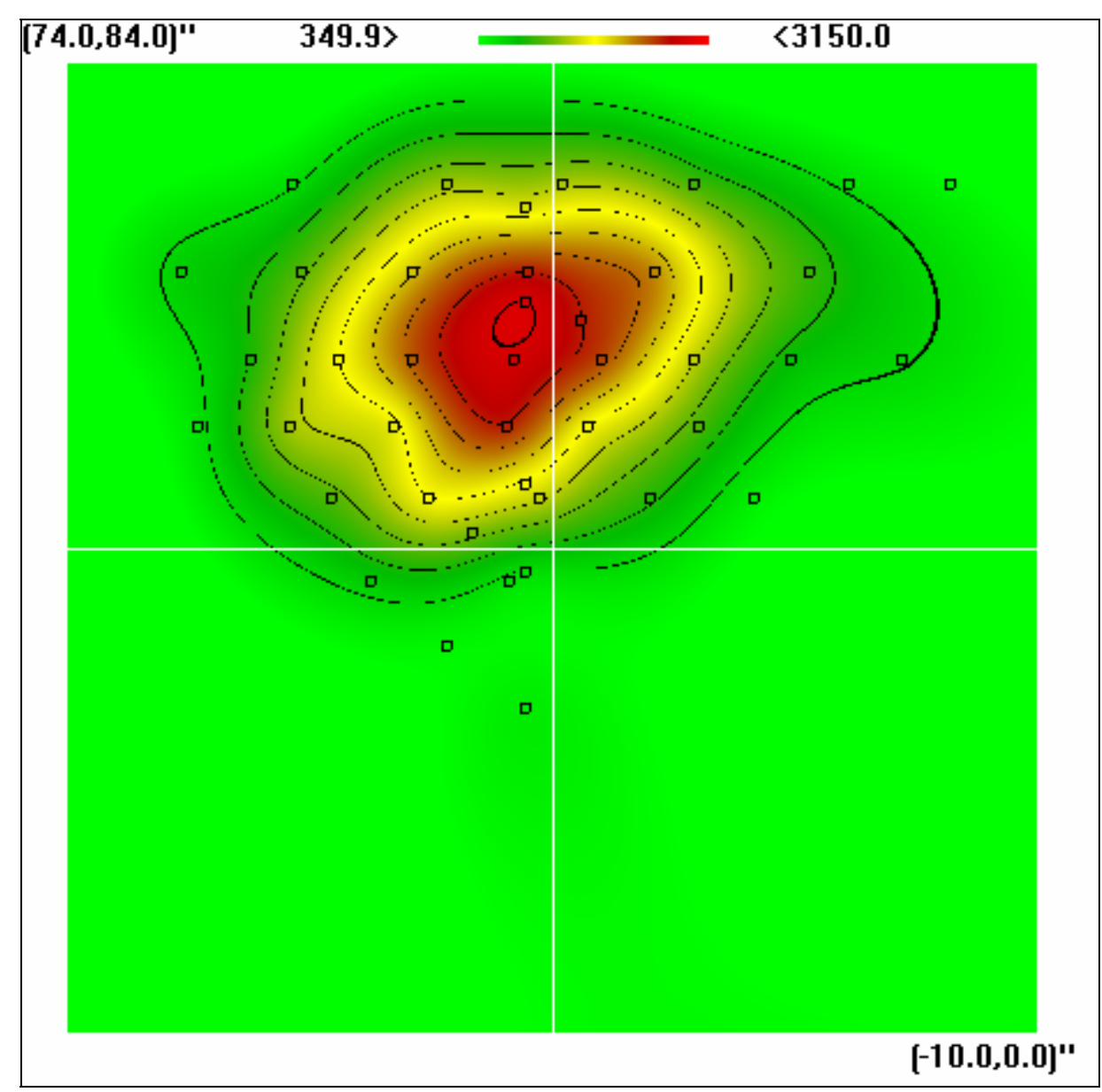

Figure $34 \mathrm{CO}_{2}$ Mapping Results for an Axial Placement of 10ft (Taken on Day 8).

(Color Scale Refers to CO2 concentration (ppm))

As can be seen in Figure 34, the outer region of the plume has made contact with the ceiling at the $10 \mathrm{ft}$ station. This contact is also apparent in the R75 case. In fact, other than added waviness and an obviously smaller $\mathrm{CO}_{2}$ scale, the plume of the I30 case has followed the same general expansion as that in the R75 case. Since the overall size and location of the plume are roughly the same for the two engine settings, the exhaust volume flow rate was considerably lower for the I30 than for R75 conditions, for any point within the $\mathrm{CO}_{2}$ field. As such, the plume will likely be more influenced by environmental variations at any given location. An example single point data set for 
similar locations (axially and cross-sectionally) of the two different engine settings is provided in Figure 35.

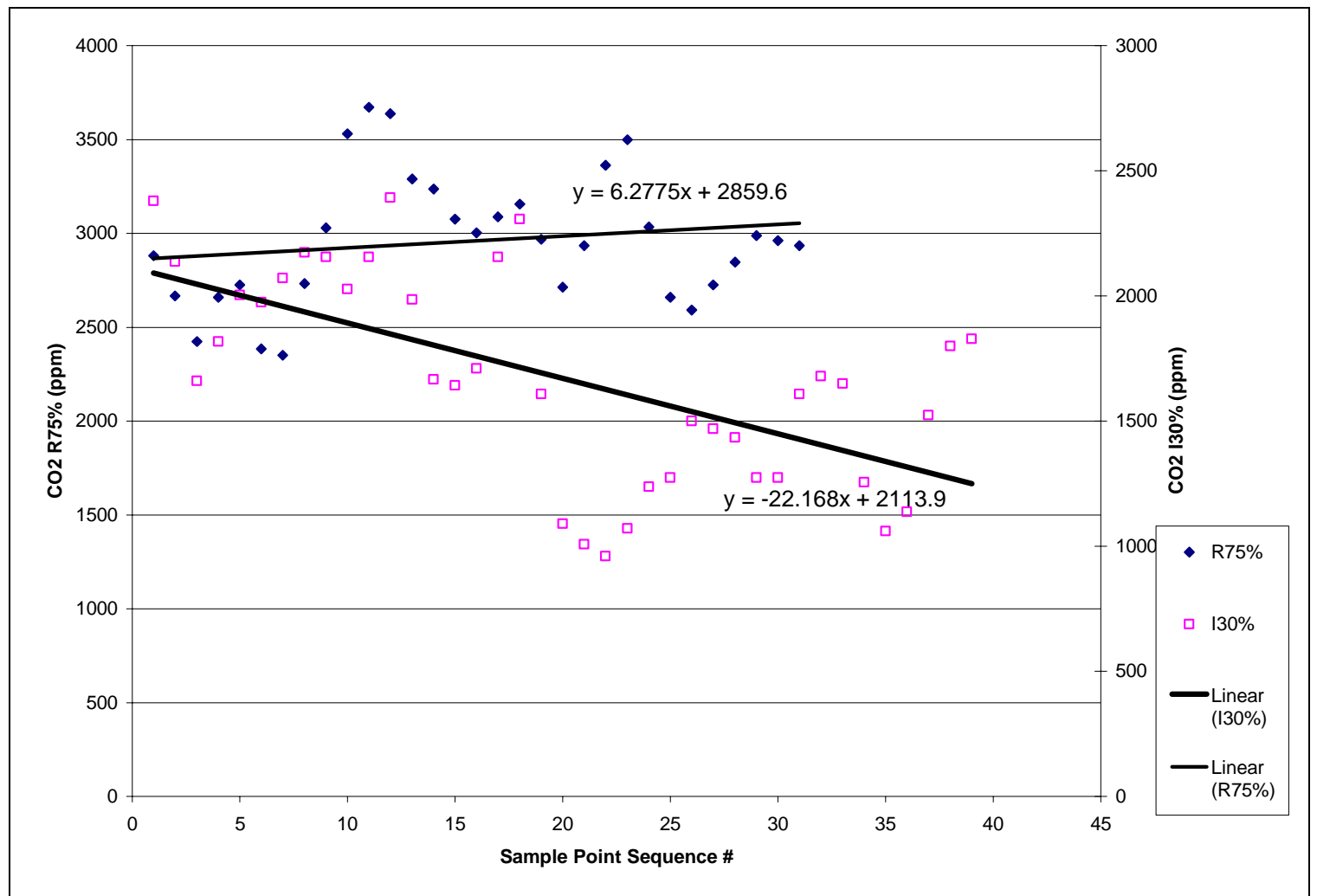

Figure 35 Example single point data used to obtain an average for both engine settings

As can be seen in Figure 35, the variation present in the data acquired for the I30 case is noticeably larger than for the R75 case. In spite of the fact that more data points were taken to form the I30 average, the slope of the linear trend-line fitted to this data is steeper. Linear trend-line slope is an indication of the legitimacy to form a single value average to a data set. As the absolute value of the slope increases, single value averaging becomes less appropriate. The ratio of the I30 to the R75 trend line slope is near a factor of 4 indicating that the R75 average is much more appropriate for its respective data set. Similar disagreements can be expected for other data point comparisons between the two modes. As such, the maps generated for the R75 case are observed to be much smoother 
than their I30 counterparts. This might be resolved in future tests, by averaging longer and / or introducing instantaneous exhaust maps for engine modes that produce lower exhaust flow rates.

Figure 36 shows the $\mathrm{CO}_{2}$ mapping results made at an axial placement of $25 \mathrm{ft}$ on day 10.

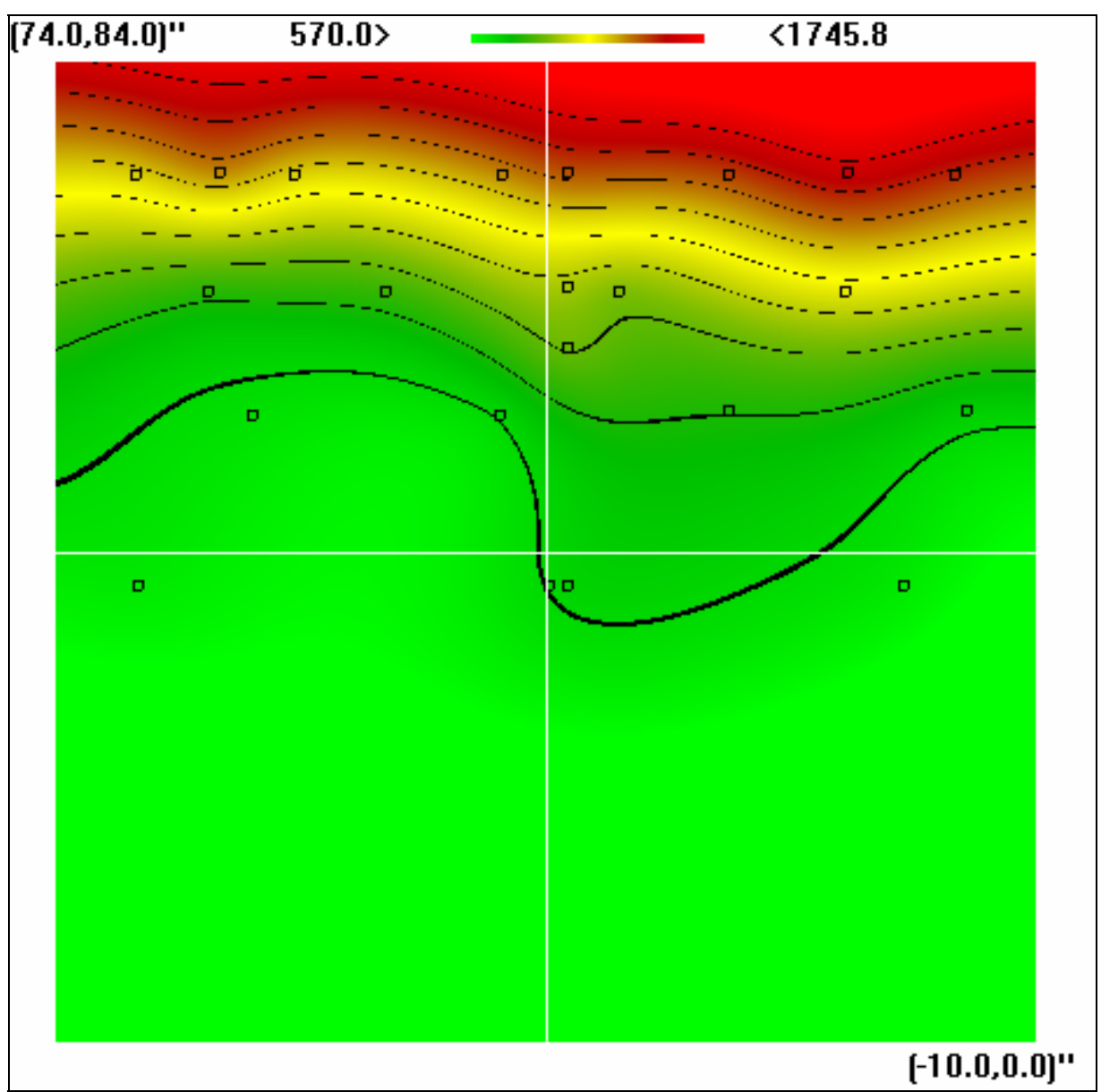

Figure $36 \mathrm{CO}_{2}$ Mapping Results for an Axial Placement of 25ft (Taken on Day 10). (Color Scale Refers to CO2 concentration (ppm))

Again, the $\mathrm{CO}_{2}$ gases have stratified as in the R75 case. At this relatively distant station, $\mathrm{CO}_{2}$ levels still vary by more than $300 \%$ from peak to background levels. In addition, due to similarity in $\mathrm{CO}_{2}$ scale with that of the $10 \mathrm{ft}$ station, it is not expected that the plume found at 25 feet has made contact with the walls of the tunnel. 
Figure 37 shows the $\mathrm{CO}_{2}$ map made at 100 feet on day 10 .

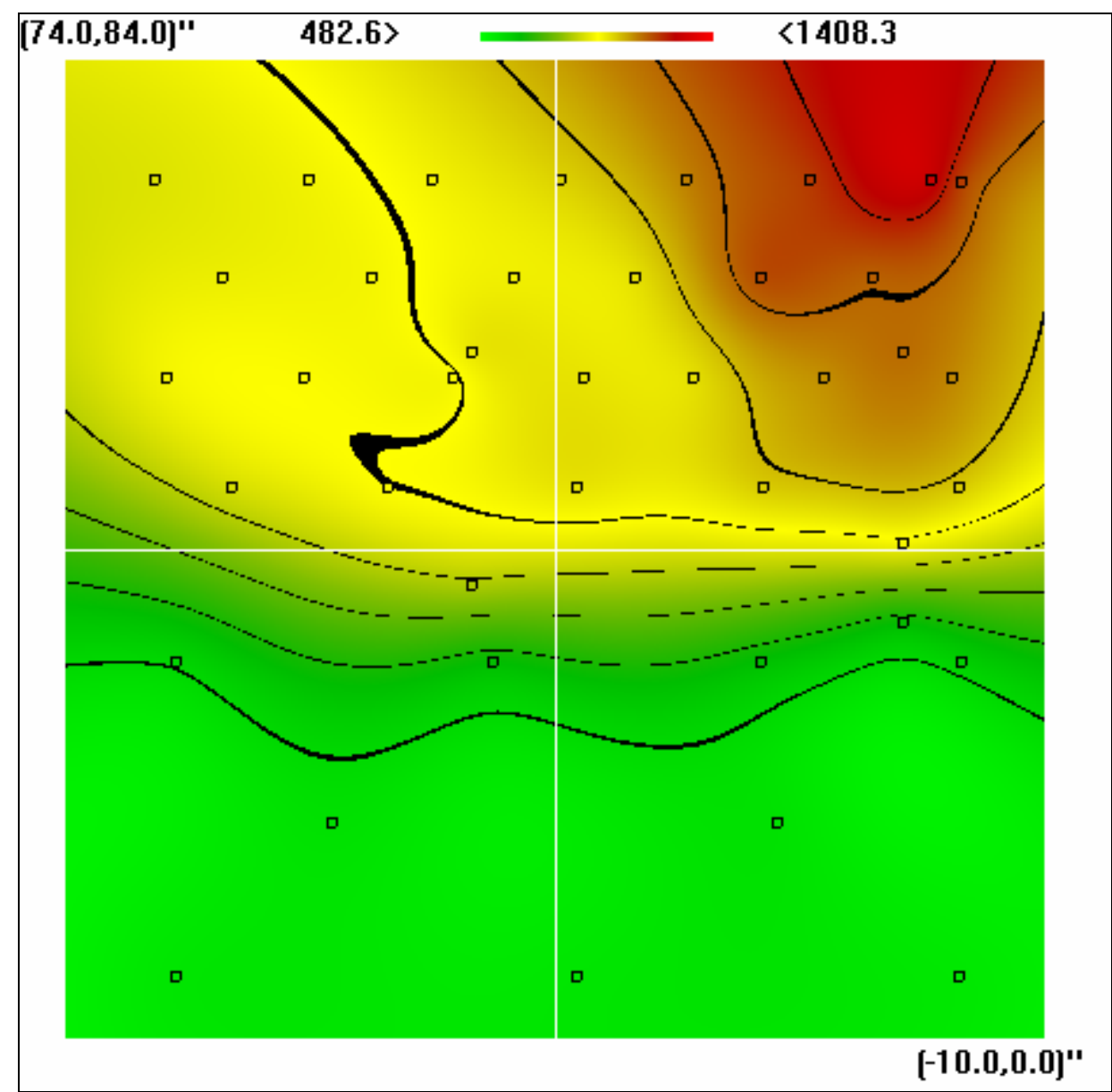

Figure $37 \mathrm{CO}_{2}$ Mapping Results for an Axial Placement of 100ft (Taken on Day 10).

(Color Scale Refers to CO2 concentration (ppm))

This station shows good agreement between data samples but appears to be a less stratified version of the 25 foot map. However, the scale of $\mathrm{CO}_{2}$ is roughly similar to that of the 25 foot station. In addition, the $\mathrm{CO}_{2}$ variations within this map are still near the $300 \%$ observed at the 25 foot station. Due to arguments described later, it is suspected that the door may have been open during the majority of this test. This issue may be a possible reason for the rearrangement of the stratification contours at this station.

Figure 38 shows the $\mathrm{CO}_{2}$ mapping results made at an axial placement of 125 feet on day 9. 


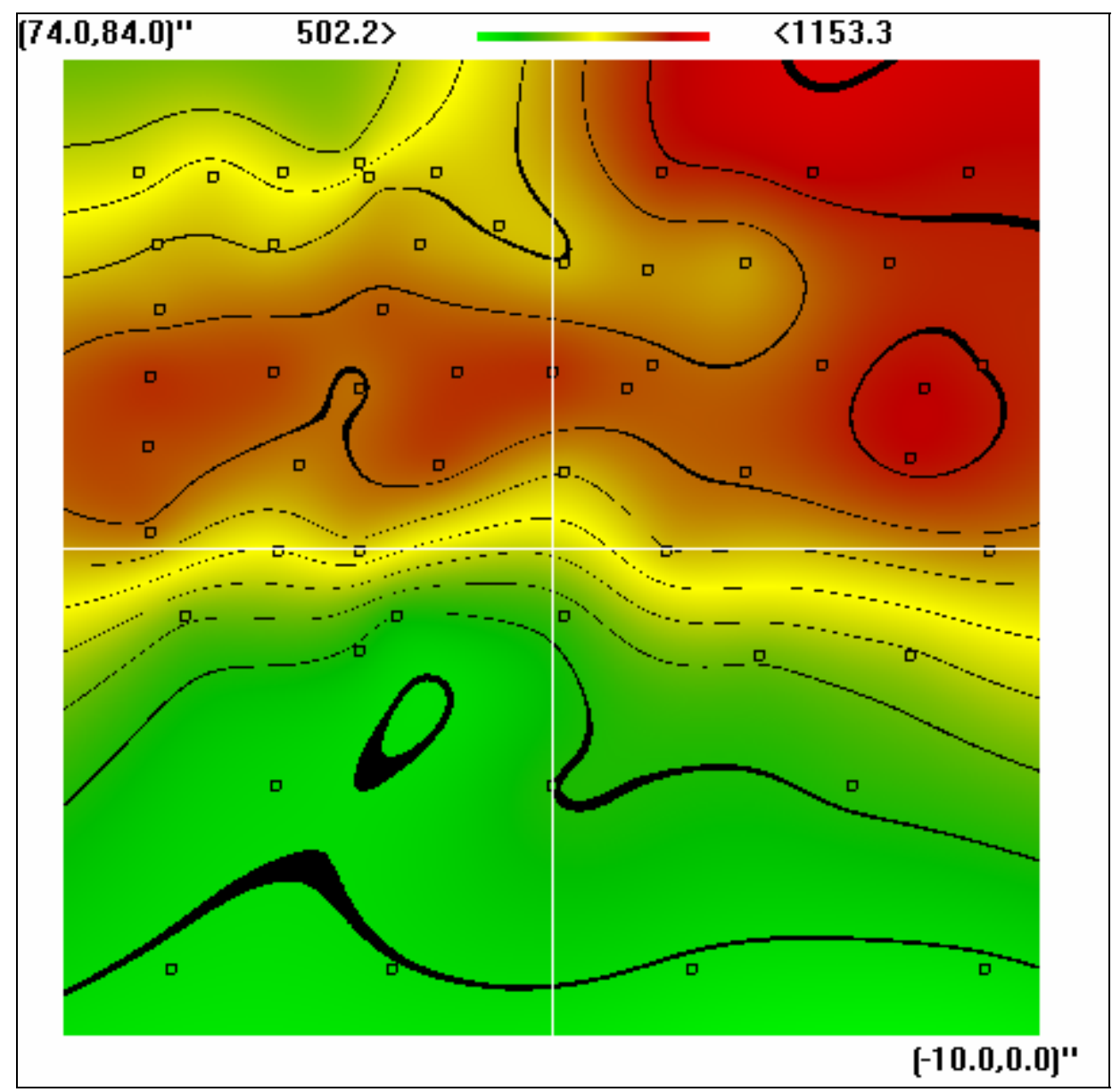

Figure $38 \mathrm{CO}_{2}$ Mapping Results for an Axial Placement of 125ft (Taken on Day 9).

(Color Scale Refers to CO2 concentration (ppm))

The map of Figure 38 is vastly different from any other map observed at this relatively distant station. The region appears to form a complex protrusion of exhaust gases from the right to the left of the tunnel. Sample points in the upper left region of the map were taken at the beginning and end of the scan and demonstrate relatively good agreement over time. Because of this, if the tunnel door had been open during this time, it appears that it must have been open the whole time. Due to the sensitivity of the plume to environmental changes under this engine mode of operation, maps of this complex nature may be expected for downstream stations where gas levels are low. This reasoning might 
explain the sudden unpredictable shift in the form of the plume from stations beyond 25 feet.

Figure 39 shows the $\mathrm{CO}_{2}$ mapping results made on day 9 at an axial placement of 150 feet.

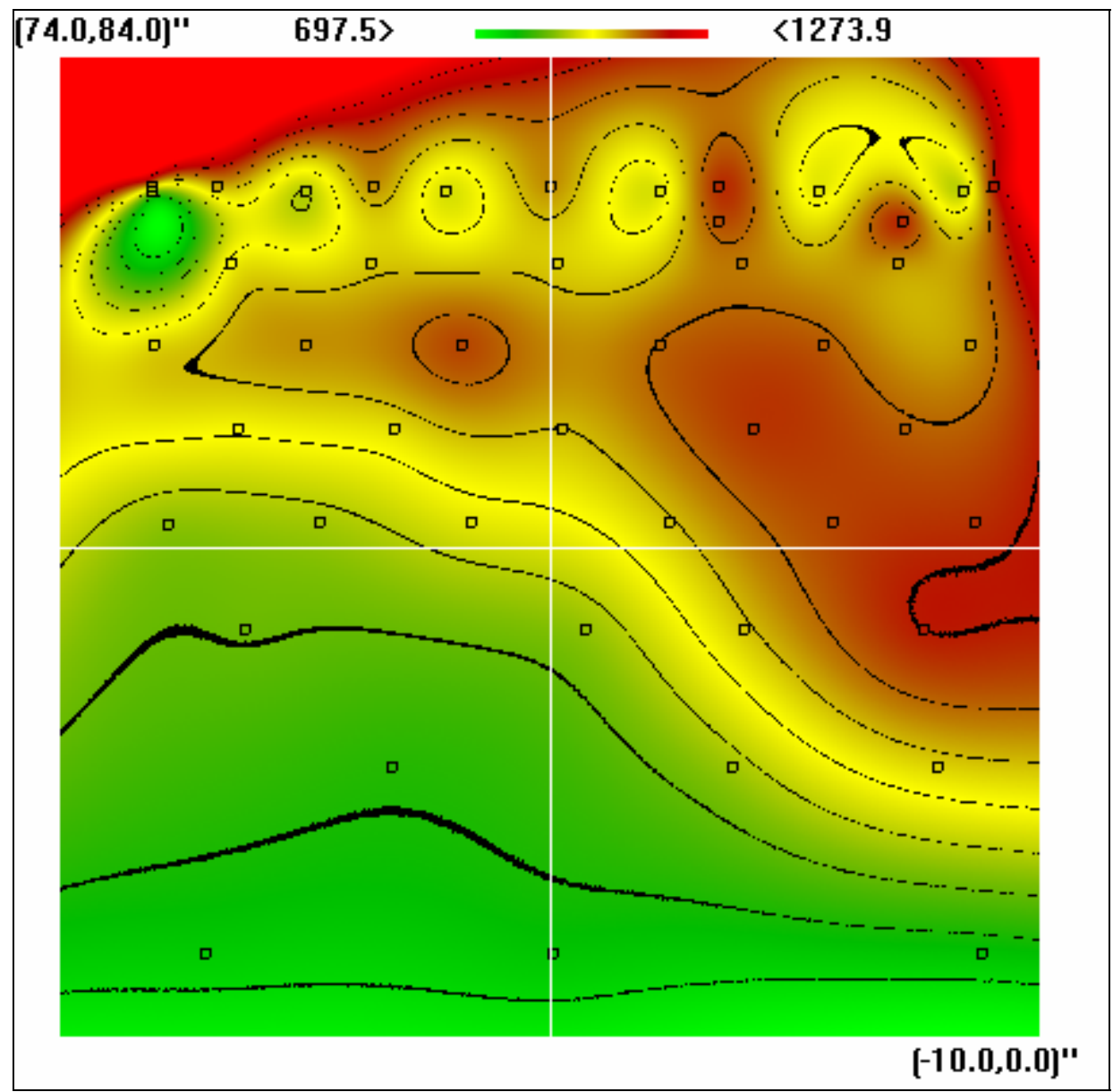

Figure $39 \mathrm{CO}_{2}$ Mapping Results for an Axial Placement of 150ft (Taken on Day 9).

(Color Scale Refers to CO2 concentration (ppm))

The map of Figure 39 appears to demonstrate inconsistency in the sample points acquired in the upper regions of the tunnel. This inconsistency was found to be the result of an open tunnel door during testing. Figure 40 demonstrates the same map if these suspect points are removed. 


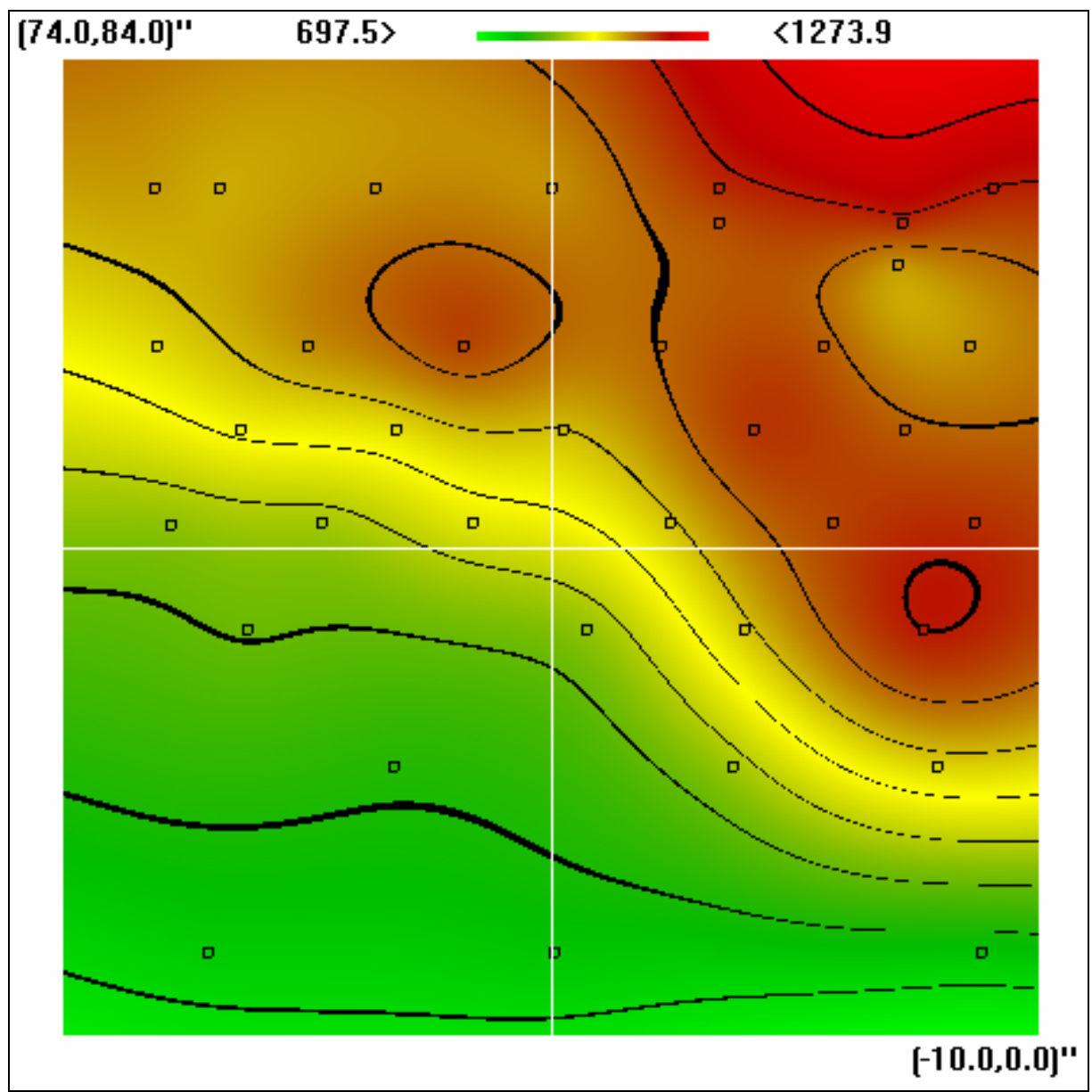

Figure $40 \mathrm{CO}_{2}$ Mapping Results for an Axial Placement of 150ft (Taken on Day 9) With Suspected “Bad” Points Taken Out. (Color Scale Refers to CO2 concentration (ppm))

Removing suspect points appears to produce a more agreeable map in Figure 40. This map is more similar to that observed in the R75 case. Unlike in this particular case, the certain cause of prior map variations is unknown. However, based on the modified results shown in Figure 40, it is believed that they are the result of similar unstable environmental conditions.

Testing results could have benefited greatly by maintaining consistent environmental factors. The observance of more agreeable data in R75 case is likely due to the momentum of high exhaust output overriding fluctuating environmental influences. This is also evident in the I30 case within the larger concentration regions of the initial plume 
stations. To determine the influence of an opened or closed door on the collection of a single point $\mathrm{CO}_{2}$ average, a single case study was performed and is summarized in Figure 41.

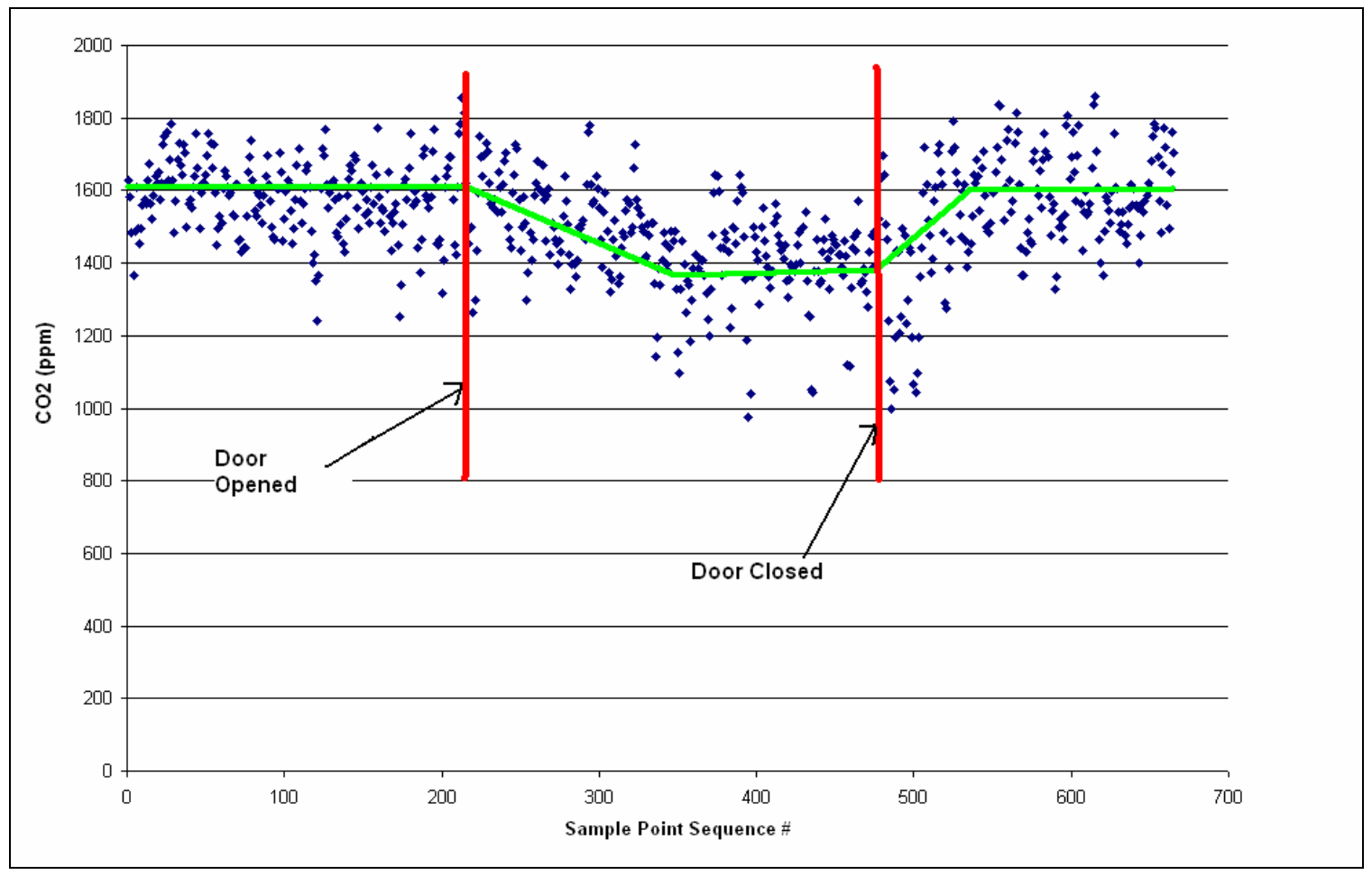

Figure 41 Effect of Door Opening on the Collection of a Single Point Average.

Figure 41 demonstrates that opening the tunnel door can significantly affect sample point averaging. In addition, it can take as long as 5 minutes for a point to fully feel the affect of this type of event. This can severely inhibit mapping results, as a full map usually only required $\sim 15$ minutes. Due to time limitations, lower concentration effects were not determined explicitly. However, they were observed, in cases where the door was known to be opened, to change by as much as $700 \mathrm{ppm}$. This variation is critical to the delicate nature of downstream stations as apparent in Figure 39 and Figure 40. 


\subsubsection{Summary}

Throughout the remainder of this study, the $\mathrm{CO}_{2}$ maps discussed in the previous sections will be used to pair dilution ratios with collected aerosol size distribution data. The dilution ratio (DR) assigned to a given sample will be calculated as.

$$
\mathrm{DR}=\left(\mathrm{CO}_{2_{\text {Raw }}}-\mathrm{CO}_{2_{\mathrm{BckGrnd}}}\right) /\left(\mathrm{CO}_{2_{\text {Map }}}-\mathrm{CO}_{2_{\mathrm{BckGrnd}}}\right) \quad \text { Equation } 45
$$

Maps will also be provided with all aerosol data as an indication of sample location within the plume. This is particularly necessary when referring to large dilution ratios which may be the result of a sample not physically present in the plume.

\subsubsection{Temperature}

The following section describes the cross-sectional temperature maps taken during this study. These maps demonstrate the buoyant potential of the plume to rise toward the ceiling of the tunnel. Due to their high similarity to the $\mathrm{CO}_{2}$ maps of the previous section, the bulk of these maps will be placed in Appendices A and B. Figure 42 demonstrates a typical temperature map taken at a 10 foot axial placement under R75 operation. 


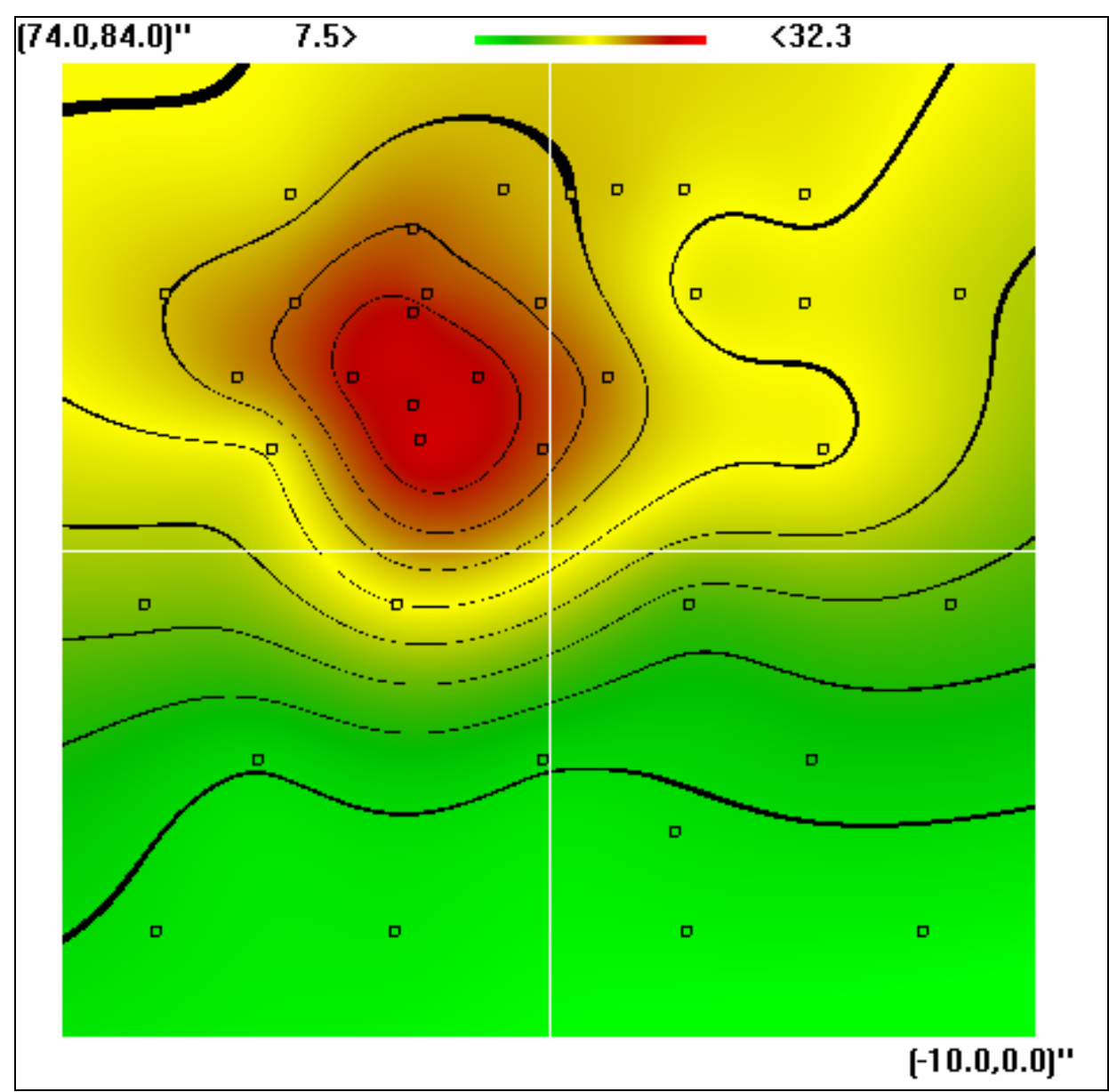

Figure 42 Temperature Mapping Results for an Axial Placement of 10ft (Taken on Day 7).

(Color Scale Refers to Temp. (C))

As can be seen in Figure 42, the temperature field appears to spread out and begin stratification much sooner than the corresponding $\mathrm{CO}_{2}$ field of Figure 25. This is most likely due to the cumulative transfer of heat from the plume to the tunnel ceiling. As such, additional waviness in this map may originate from the unsteady accumulation of heat in the tunnel ceiling.

Figure 43 shows an example of a downstream temperature map taken at 150 feet under I30 operation. 


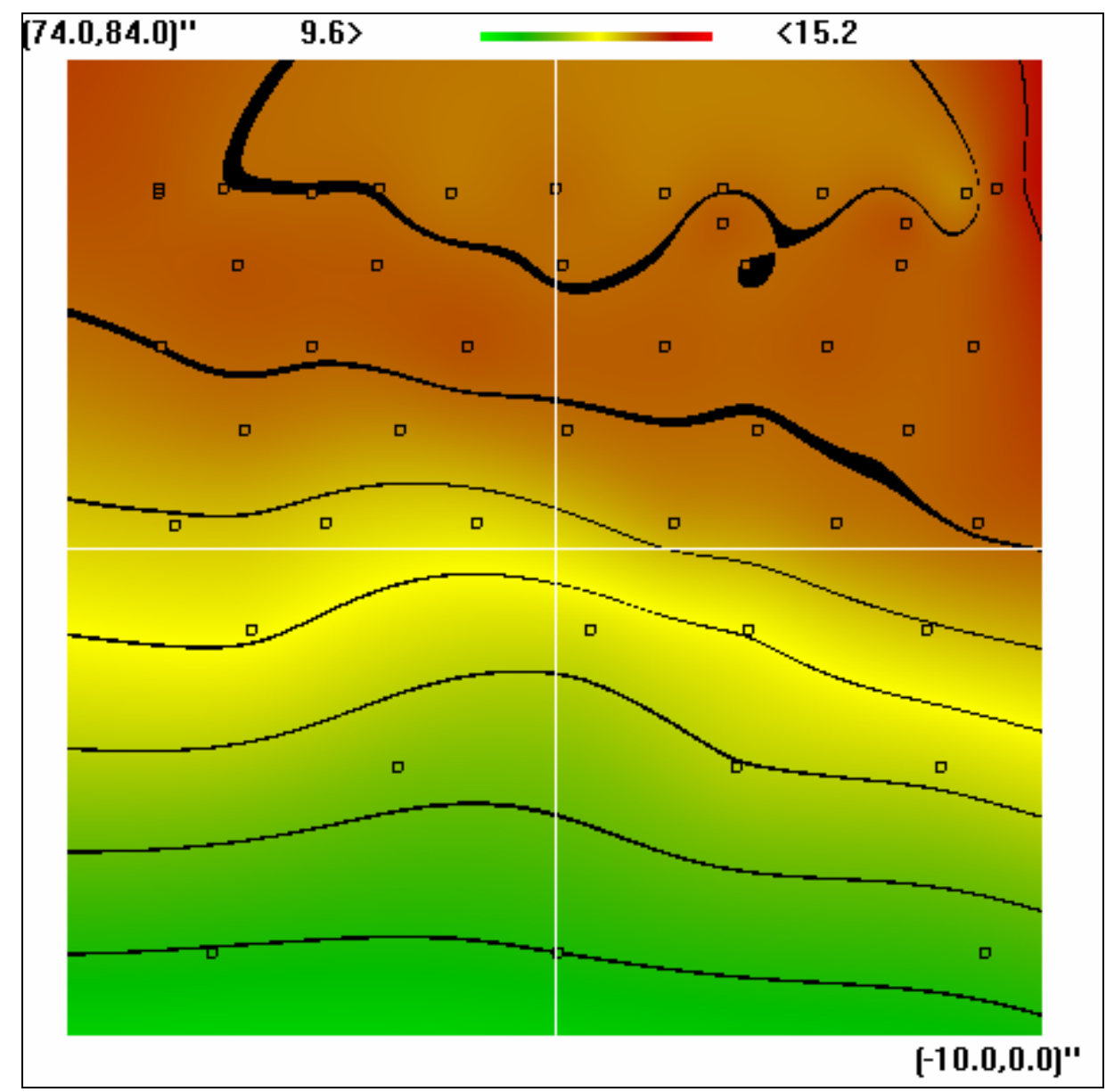

Figure 43 Temperature Mapping Results for an Axial Placement of 150ft (Taken on Day 9).

(Color Scale Refers to Temp. (C))

As can be seen in Figure 43, the temperature field has responded less to the door being opened than the corresponding $\mathrm{CO}_{2}$ field of Figure 39. In addition, spatial temperature variations are still significant enough to measure for downstream stations.

\subsubsection{Axial Velocity}

An anemometer was attached to the probe system during both modes of engine operation. The resulting data were stored independently, on a data logger, as a continuous array for each day's entirety. The data were later extracted and paired with the coordinates of sampled data points through time comparisons. Due to dependability 
issues of the logging system, only one sample of an axial velocity map will be provided. Figure 44 demonstrates the axial velocity mapping made at the 10 foot station on day 8 .

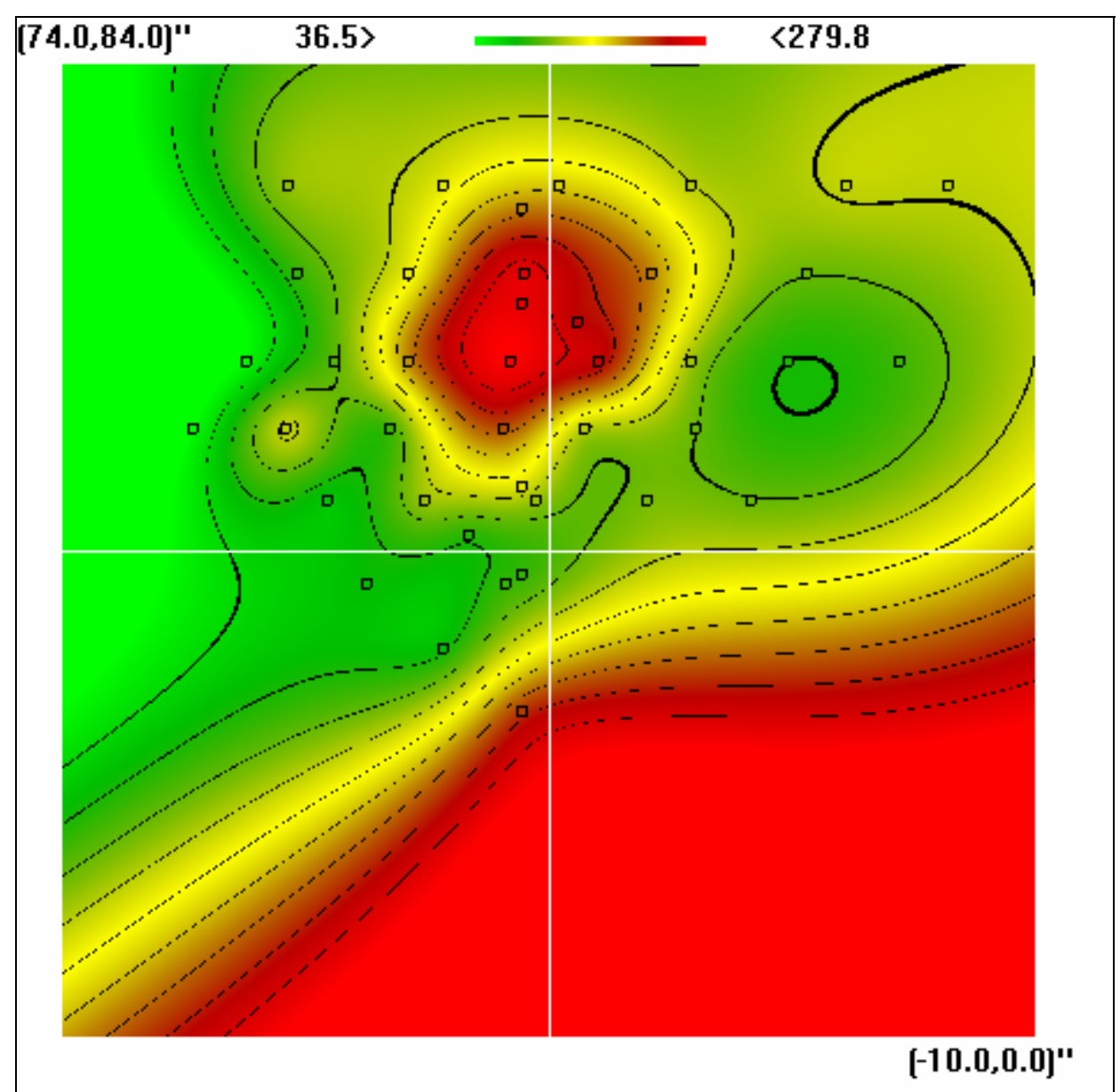

Figure 44 Axial Velocity Mapping Results for an Axial Placement of 10ft (Taken on Day 8).

(Color Scale Refers to Axial Velocity (ft/min))

As can be seen in Figure 44, the plume has a significantly larger axial velocity than the dilution air at the 10 foot station. With such a wide range of axial velocities present at this moderately distant cross-sectional station, Figure 44 demonstrates the complexity in addressing aerosol residence times for naturally mixed plumes. This complexity is worsened by the absence of velocity data in the immediate vicinity of the exhaust outlet due to anemometer sensitivity to heat. As such, throughout the remainder of this text, 
residence will only be discussed in terms of axial distance. Further studies would be required to fully track the exact exhaust path history throughout the tunnel.

\subsubsection{Aerosol Concentration}

Aerosol concentration was used as a qualitative basis to determine aerosol agreement with other scalar maps (primarily $\mathrm{CO}_{2}$ ). In addition, aerosol concentration provided information as to whether SMPS instrumentation would peak out in certain regions of the plume. Figure 45 demonstrates an example aerosol concentration map taken on day 7 at an axial placement of 10 feet.

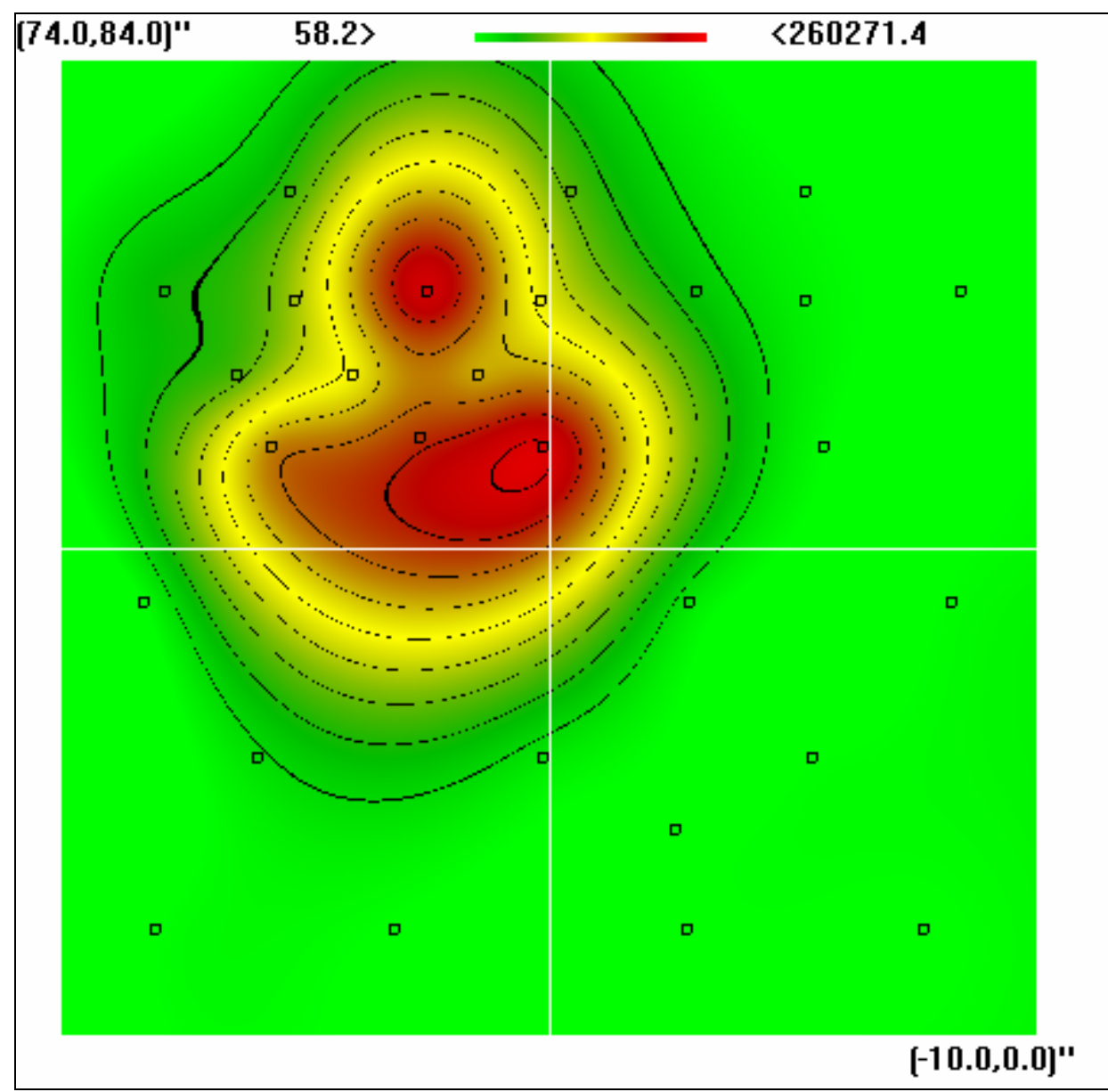

Figure 45 Aerosol Concentration Mapping Results for an Axial Placement of 10ft (Taken on Day 7).

(Color Scale Refers to Aerosol Concentration $\left(\# / \mathrm{cm}^{3}\right)$ ) 
As can be seen in Figure 45, variation in aerosol concentrations are quite large and in favor of the plume center. The variations present in this map may be the result of coupled aerosol dependence on dimensions of the plume which have dissimilar expansion patterns.

\subsubsection{Axial Development and Dilution throughout Space}

The bulk of this work was directed towards fine cross-sectional mapping with resolution in the axial dimension being more limited. Axial plume development can be seen as a sequence of these cross-sectional maps. A more global point of view can be obtained through the addition of the axial dimension to the mapping software. Figure 46 demonstrates the $\mathrm{CO}_{2}$ field obtained by a vertical axial cut through the center of the test region from 3 to $\sim 10$ feet. 


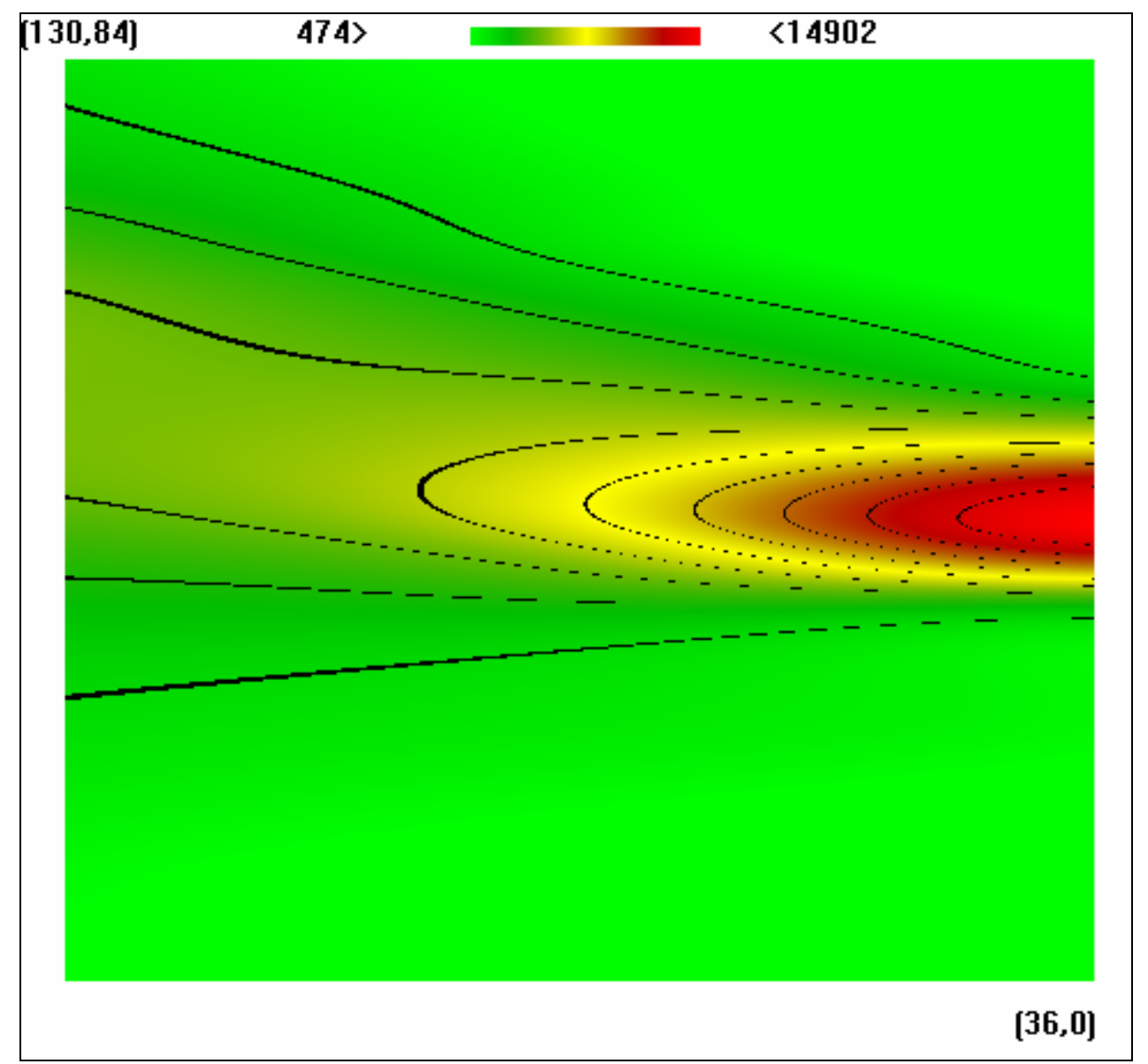

Figure 46 Example Vertical Axial Cut through Tunnel Center (Coordinate pairs represent axial and vertical distance in inches. The color scale represents $\mathrm{CO} 2$ concentration (ppm))

As can be seen in Figure 46, $\mathrm{CO}_{2}$ and hence dilution values do not only repeat themselves within a given cross-section but also axially. As such, constant levels of $\mathrm{CO}_{2}$ define three dimensional surfaces throughout the plume field. In addition, surfaces created by relatively high $\mathrm{CO}_{2}$ levels will close upon themselves at a given axial location, as necessary since certain levels of $\mathrm{CO}_{2}$ are not observed in the stratification layers downstream. An example of such a surface is provided in Figure 47 taken from data acquired on days 5 through 7 defined by a dilution ratio of 27. 


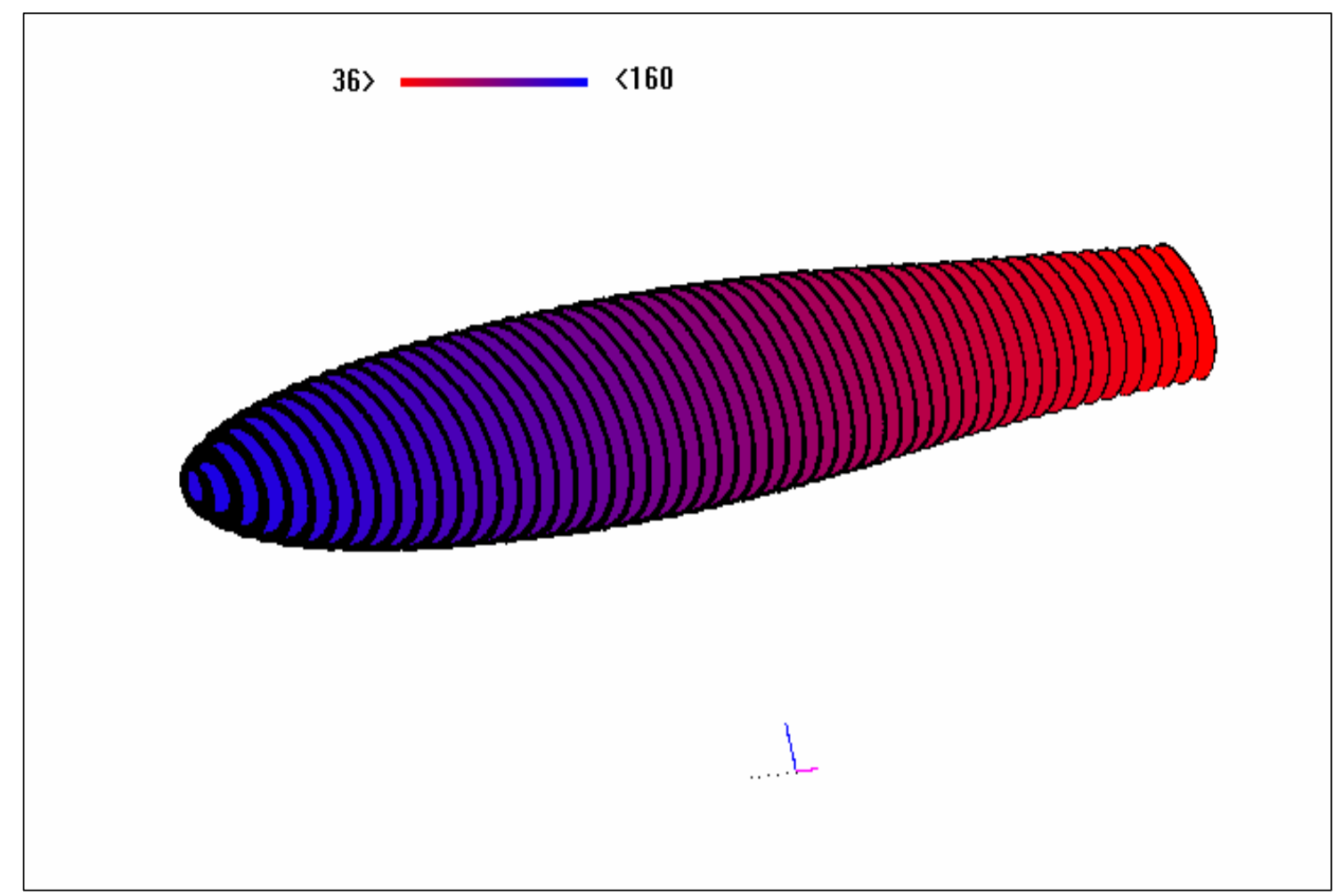

Figure 47 Example Constant Dilution $(D R=27)$ Shell Taken from Test Days 5 through 7. (the color scale refers to axial tunnel distance in inches)

As shown in Figure 47, dilution surfaces generated by relatively large $\mathrm{CO}_{2}$ levels will close at a certain axial distance from the exhaust source. Surfaces generated by relatively low $\mathrm{CO}_{2}$ values will begin a conical path (as in Figure 47) which open to a flat surface in the stratification layers down stream. This opening effect is due to the affinity of hot exhaust gases with the tunnel ceiling through buoyancy and tunnel slope.

As the plume rises, the conical surface will make contact with the ceiling. The inner regions of the cone continue to rise, splitting the upper part of the cone into a distorted plane. As the process continues, the plane becomes more and more flat as it spreads to the walls of the tunnel. If further dilution occurs, these surfaces will eventually collapse to a single dilution solid representing fully mixed flow. In truly unconstrained environments these open conical surfaces will not exist. In these situations, constant $\mathrm{CO}_{2}$ 
contours will always form surfaces of the type in Figure 47. Due to the massive amount of time required to generate these surfaces, the splitting case is only shown from $\sim 10$ to 20 feet in Figure 48. A full pictorial example is provided in Figure 49.

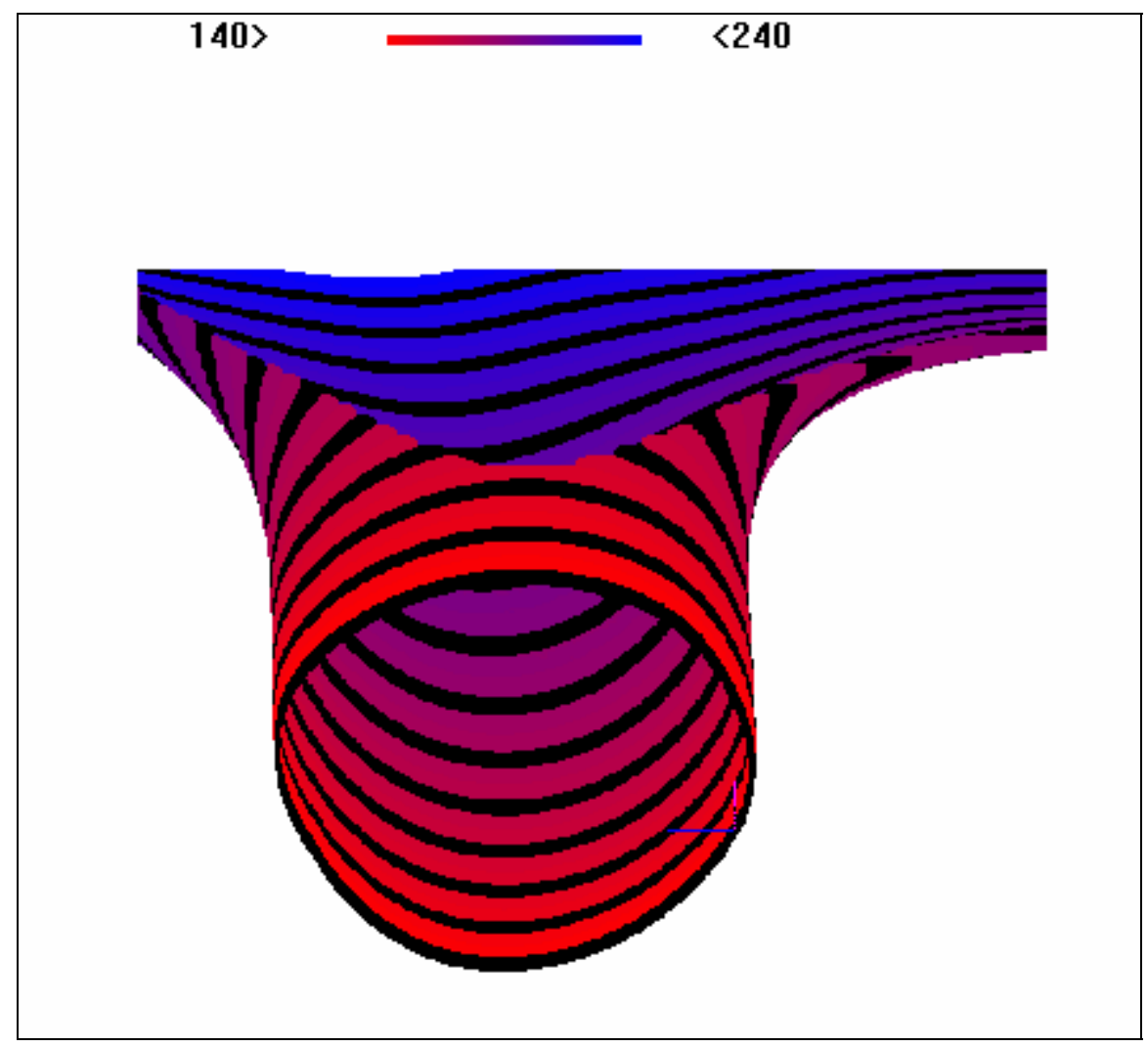

Figure 48 Example of a Constant Dilution Surface that Splits into a Stratified Layer at the Tunnel Ceiling. (Generated from data taken on test days 5 through 7. Color scale refers to axial distance (inch)) 


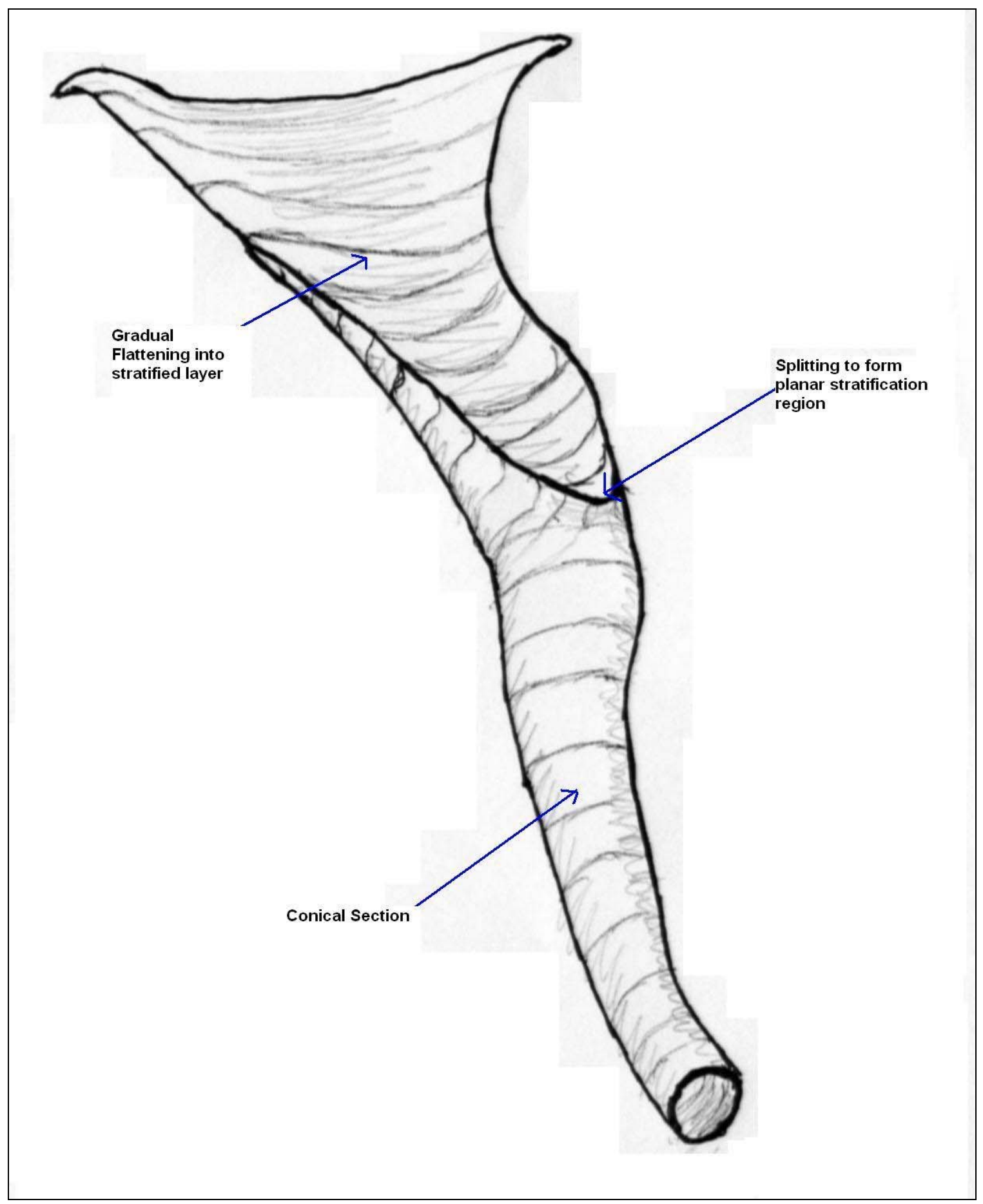

Figure 49 Illustration of a Constant Dilution Surface Involving a Transition into Stratification 


\subsubsection{Combined Development of $\mathrm{CO}_{2}$, Temperature, and Aerosol Concentration}

The approach employed during this study was based on using select high resolution exhaust maps to selectively acquire trends in more time expensive exhaust data. To track an exhaust quantity by rigorously mapping another, which may not be physically connected to the tracked quantity, requires knowledge of the expansion pattern relationship that the two quantities share. In the case of this study, it is necessary to know whether contours of $\mathrm{CO}_{2}$, temperature, and aerosol concentration translate into contours of aerosol size distribution. Without this knowledge, it cannot be said that the subsequent trends to be observed in the aerosol size distribution data were completely resolved by following the contours of the above mentioned maps. Unfortunately, this knowledge would require an unpractical amount of precision in the time expensive aerosol size distribution samples. Some information into this subject might be acquired by comparing the expansion patterns of those quantities that were mapped thoroughly (i.e. $\mathrm{CO}_{2}$, temperature, and aerosol concentration). These comparisons might at least lead to a qualitative assurance of the fullness to be found in the subsequent aerosol sized distribution data. As such, Figure 50 and Figure 51 compare the simultaneous occurrence of $\mathrm{CO}_{2}$ and temperature at regions where the plume exists in its concentric and stratified form respectively. 


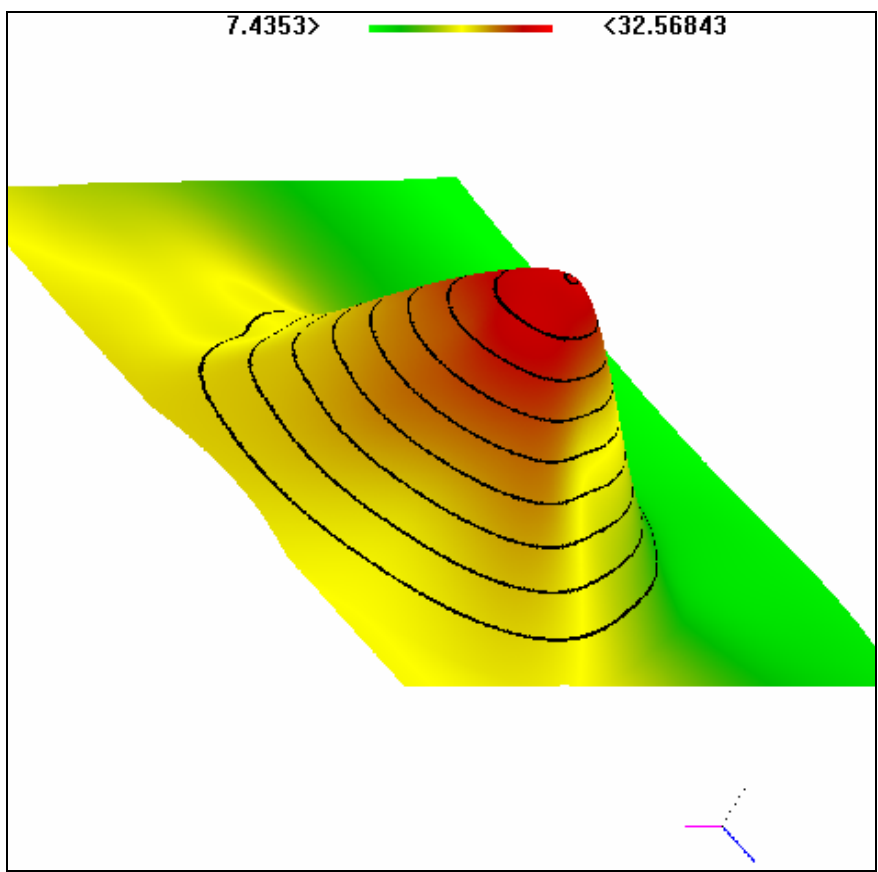

Figure 50 Simultaneous $\mathrm{CO}_{2}$ and Temperature Comparison Taken on Day 7 at a 10ft Axial Placement. (Elevation Bands Refer to $\mathrm{CO}_{2}$ contours while Color Refers to Temperature)

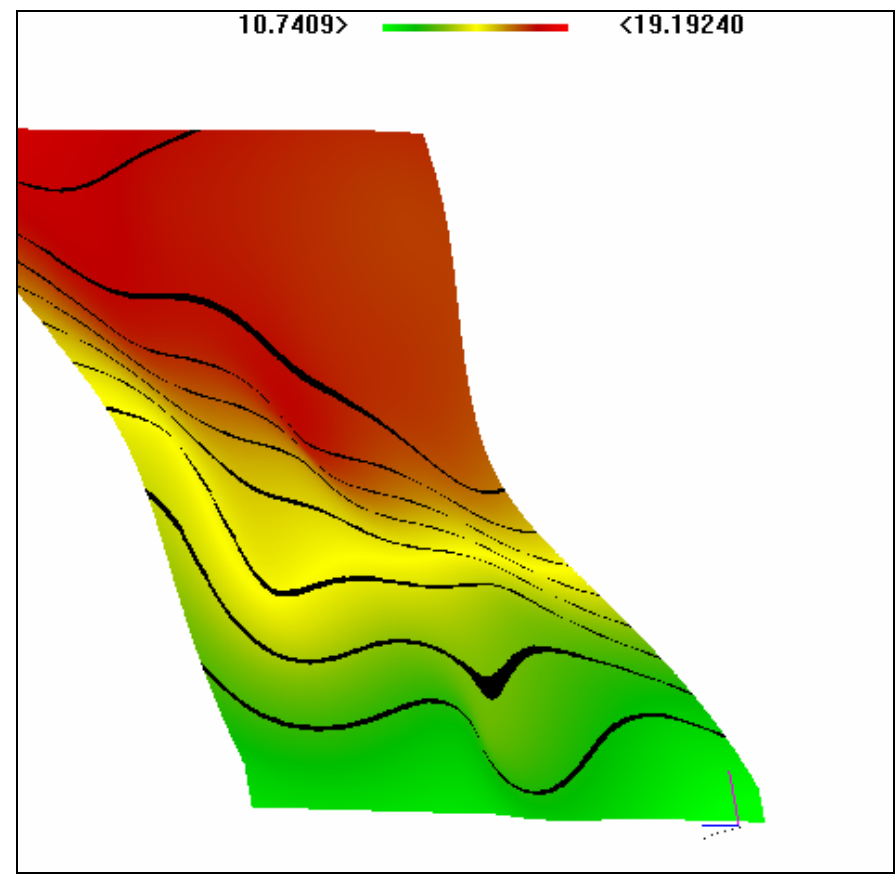

Figure 51 Simultaneous $\mathrm{CO}_{2}$ and Temperature Comparison Taken on Day 5 at a 125ft Axial

Placement. (Elevation Bands Refer to $\mathrm{CO}_{2}$ contours while Color Refers to Temperature) 
As can be seen, temperature and $\mathrm{CO}_{2}$ follow relatively similar expansion patterns within the plume (at least within a given cross-section). This similarity is more apparent for the 125ft case of Figure 51 where the flow is relatively stable and fully developed. Less agreement is observed for the expansion patterns of $\mathrm{CO}_{2}$ and temperature in the $10 \mathrm{ft}$ case of Figure 50. To demonstrate this observation further, the $10 \%$ contour bands of each data set (temperature and $\mathrm{CO}_{2}$ ) are drawn simultaneously in Figure 52.

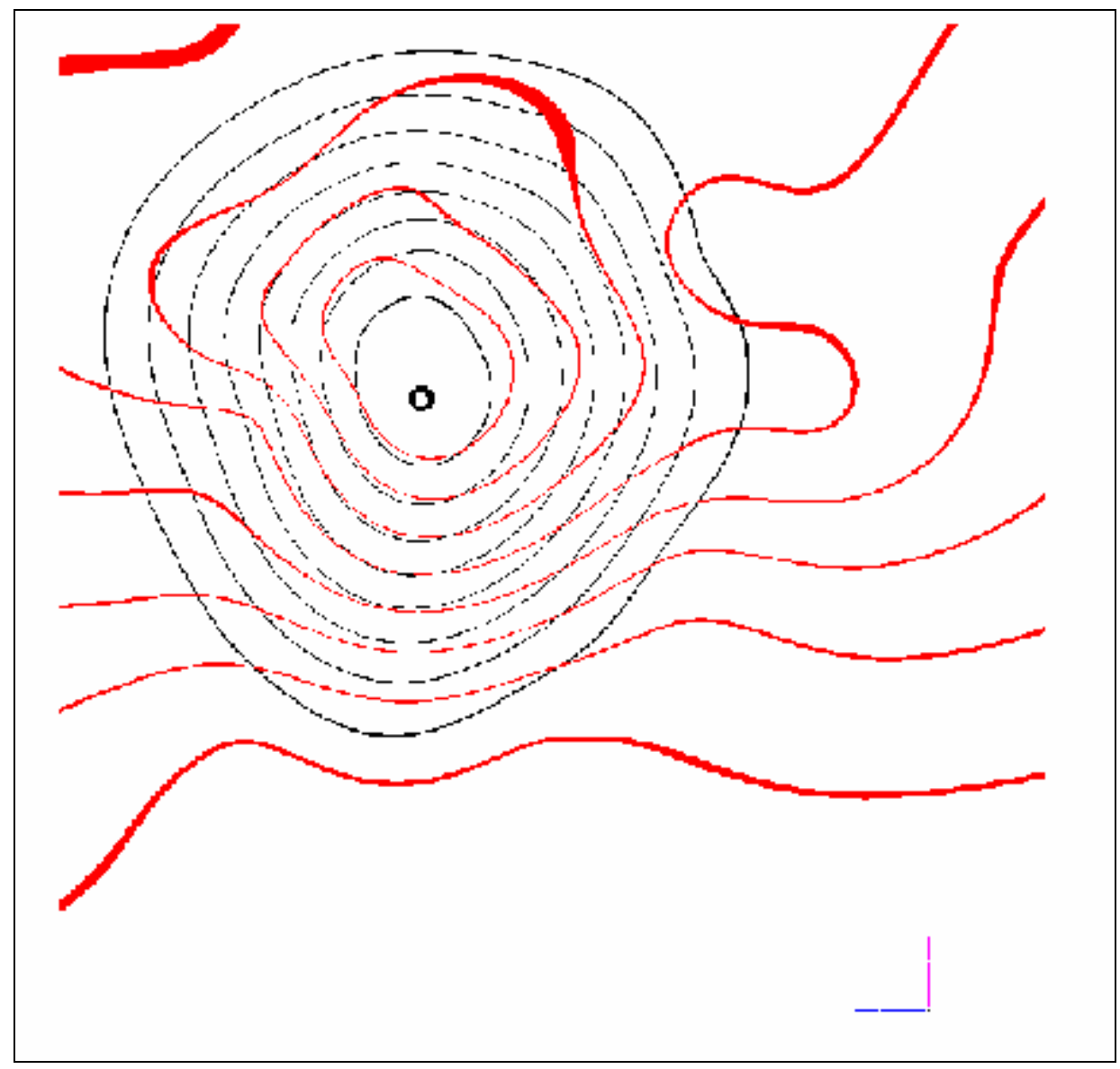

Figure 52 The Simultaneous Comparison of $\mathrm{CO}_{2}$ and Temperature Expansion Patterns Found in

Figure 50. (Black and Red Bands Represent $\mathrm{CO}_{2}$ and Temperature $10 \%$ Contours Respectively) As can be seen in Figure 52, $\mathrm{CO}_{2}$ and temperature contours cross at a number of locations. This crossing demonstrates that various internal properties of the plume are 
not bound to the same expansion patterns. This particular case appears to originate from the interaction of the tunnel ceiling with the non conservative temperature field. As the cool ceiling absorbs energy from the plume, the temperature contours will open into prematurely stratified layers. This will primarily affect the upper regions of the plume in immediate contact with the ceiling. The lower regions of the plume display relatively good agreement between $\mathrm{CO}_{2}$ and temperature expansion patterns. However, even these regions exhibit unique expansion rates which tend to propagate their differences when moving away from the plume's center.

By Figure 52, it is evident that constant dilution will not necessarily define constant exhaust properties (i.e. temperature) for a given residence time (i.e. axial station). This observation may also be true for quantities other than temperature but will require further attention from future studies. The potential variation of exhaust parameters under constant dilution and residence time may lead to undesirable complexities and or irregularities in typically metric ordered results. Figure 53 describes the temperature variations present in the $10 \%$ contour bands of $\mathrm{CO}_{2}$ taken on day 7 at an axial placement of 10 feet. 


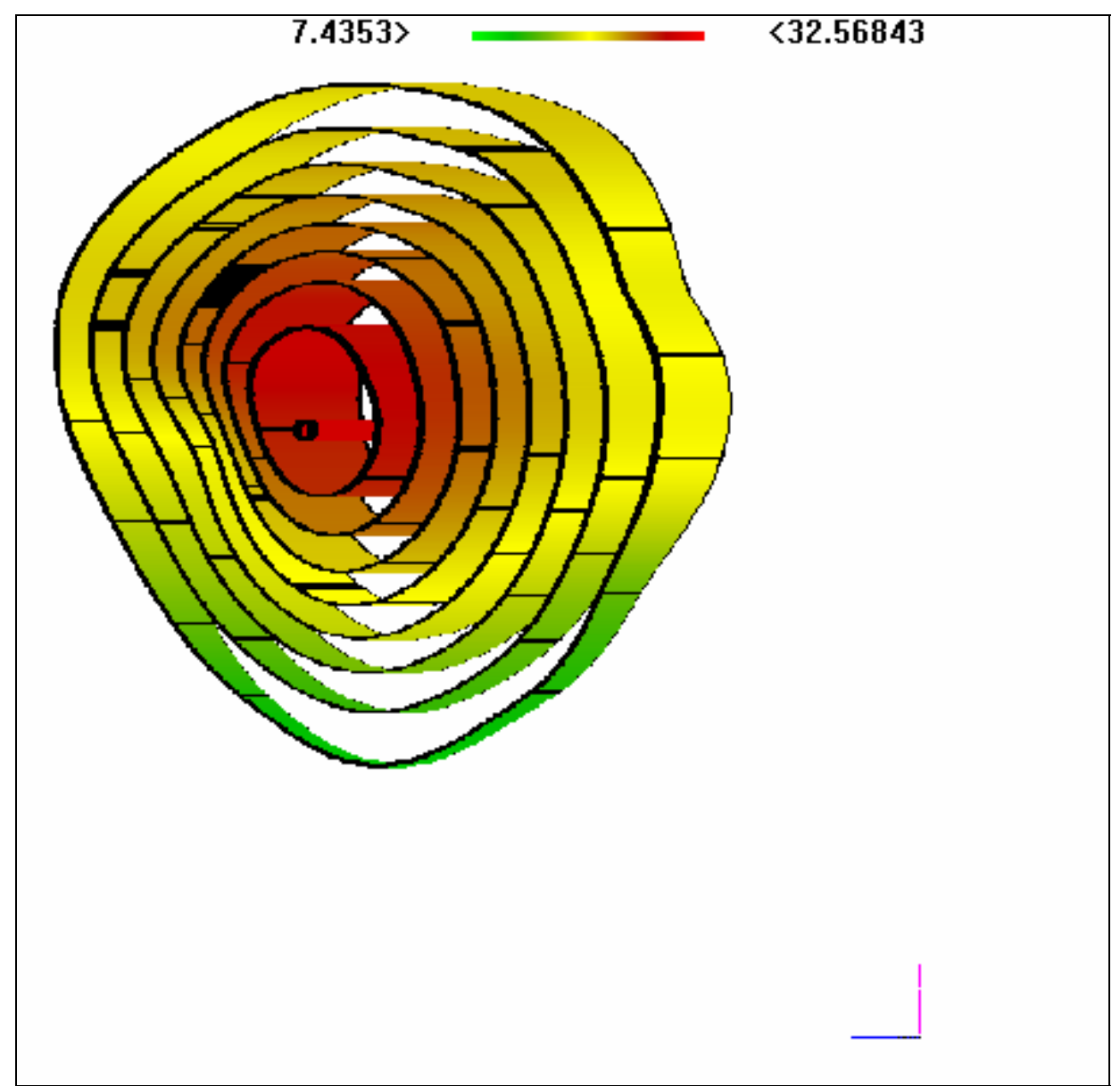

Figure 53 Temperature Variations for the $\mathrm{CO}_{2}$ Contours Taken on Day 7 at an Axial Placement of 10ft. (Color Scale refers to Temperature in C)

As can be seen in Figure 53, temperature variations are relatively large for the outer $\mathrm{CO}_{2}$ contours of the plume. In these regions, temperatures can vary by as much as 60\% (15 C) of the temperature scale.

Since obvious differences have been shown to exist between the $\mathrm{CO}_{2}$ and temperature expansion patterns of this study, it is necessary to know which, if any, of these two maps provide a better indication of aerosol size distribution development. Other maps may be used for this purpose (i.e. velocity and aerosol concentration) but the instruments chosen to record them, during the course of this study, were found unreliable. Since a direct relationship between aerosol size distribution and the maps of $\mathrm{CO}_{2}$ and temperature 
would require an unpractical amount of time, an indirect relationship might be discovered through a connection to the aerosol concentration maps which produced relatively stable results.

To understand the impact of varying $\mathrm{CO}_{2}$ and temperature on aerosol concentration, these quantities were ordered based on a uniform spatial grid placed in each of the maps of the $10 \mathrm{ft}$ axial station taken on day 7 . The resulting coordinates $\left(\mathrm{CO}_{2}\right.$ and temperature) of this data set $\left(\mathrm{CO}_{2}\right.$, temperature, and aerosol concentration) are shown in Figure 54.

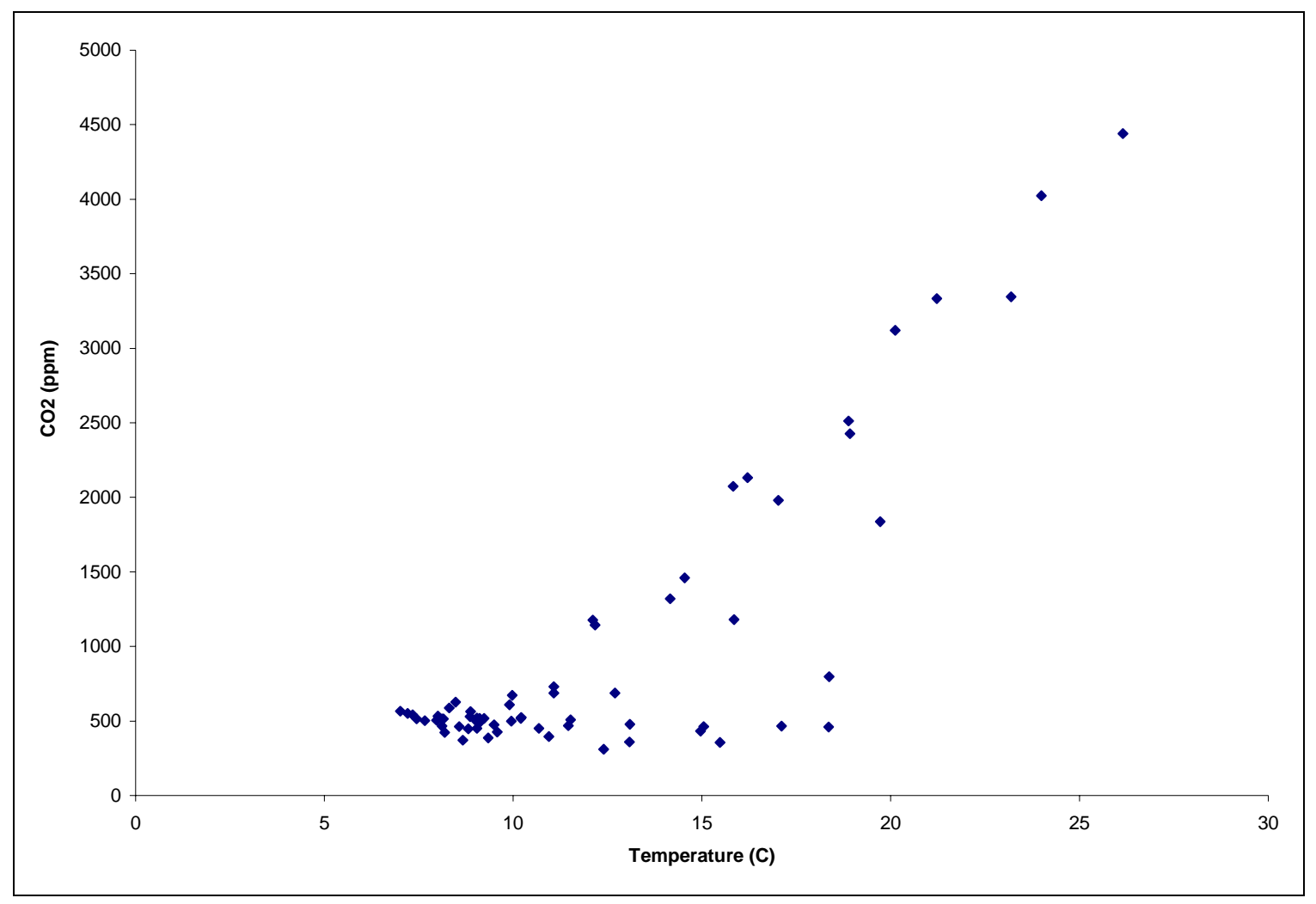

Figure 54 Resulting Scalar Coordinate Grid Obtained from a Uniform Spatial Grid. (10ft axial placement taken on day 7)

As can be seen, the bulk of these points lie at low $\mathrm{CO}_{2}$ levels as this naturally occupies more flow area and will draw in more of the uniform spatial grid locations. Fewer points will be found near lower dilutions (i.e. higher $\mathrm{CO}_{2}$ ) due to the relatively small central region of the plume. In addition, although points were equally spaced throughout the 
plume, they are not equally spaced with respect to the new coordinates. Being that each point of Figure 54 is a dependent variable of the plume, spacing of the points is left to the nature of the plume. In this case, the points spread to a triangular region whose temperature base is wide as it represents the outer regions of the plume where large variations in temperature are found to exist. Figure 55 shows the arrangement of the $\mathrm{CO}_{2}$, temperature, and aerosol concentration variables, as they occur naturally throughout the plume.

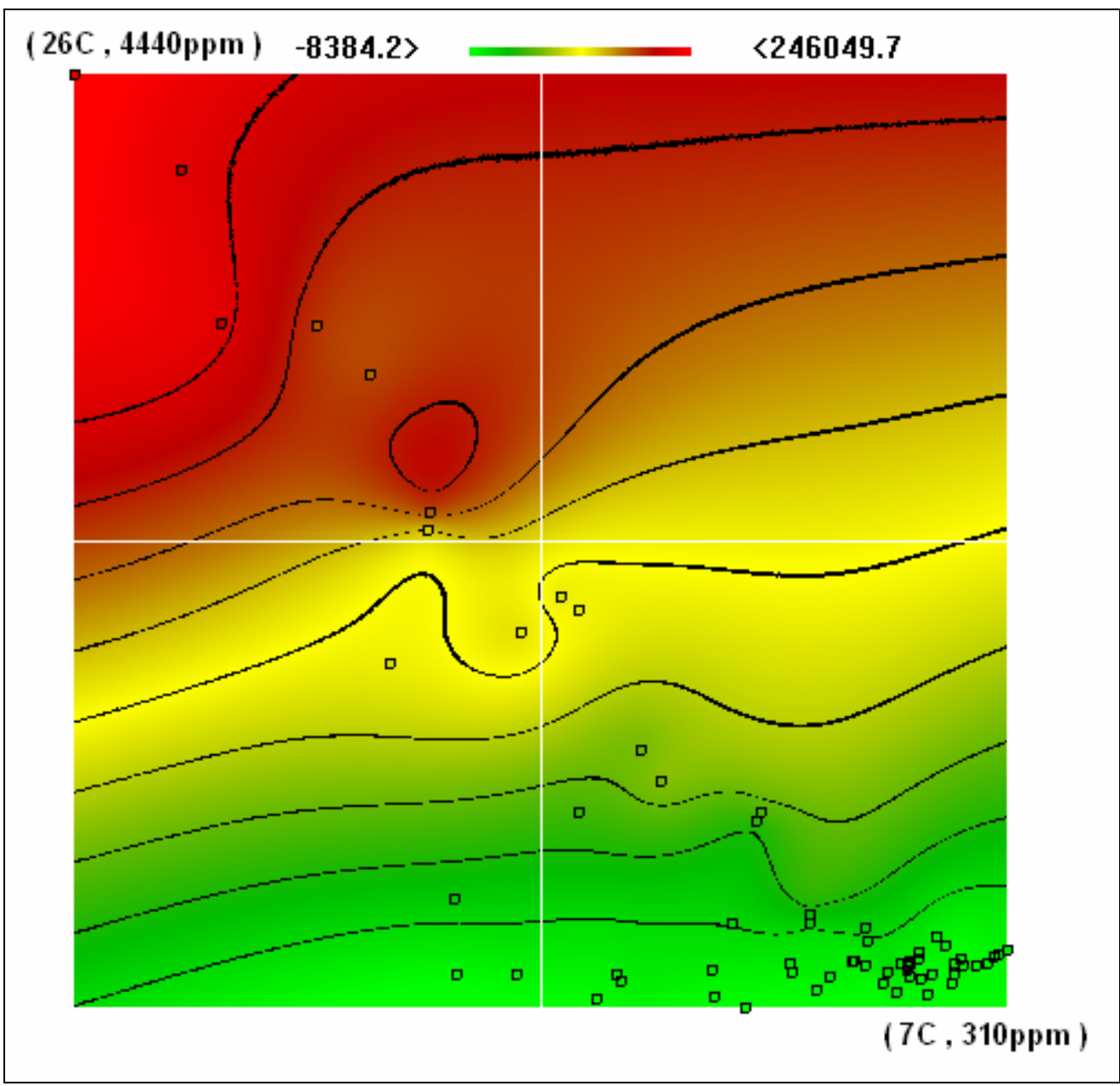

Figure 55 Aerosol Concentration Dependence on $\mathrm{CO}_{2}$ and Temperature at an Axial Placement of 10ft Taken on Day 7. (Color scale is un-calibrated and represents trends in PM concentration

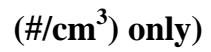


As can be seen, the contours in the Figure 55 map are generally parallel to the temperature axis. As such, aerosol concentration dependence, within the dimensions of this map, is primarily dominated by the presence of $\mathrm{CO}_{2}$.

Since the dimension of the plume evidently exceeds the dimension represented by the map, the aerosol levels observed in Figure 55 cannot be solely attributed to the listed coordinates. However, since any other exhaust influences are presumably unordered (i.e. their may not be a sequential order to other exhaust variables imbedded within the map by simply ordering $\mathrm{CO}_{2}$ and temperature), they should present themselves as irregularities with no apparent visual order. As such, the waviness present in isolated regions of the map may not be due to $\mathrm{CO}_{2}$ and / or temperature influence and may be due to the impact of other unrecorded exhaust influences. Nevertheless, due to the apparent regularity of the contours in favor of $\mathrm{CO}_{2}$, it can be expected that $\mathrm{CO}_{2}$ and likewise dilution ratio have more of an influence (in terms of a test metric) on aerosol concentration than does temperature. This may not be true when comparing the influence of other exhaust quantities and will require more attention from future studies. In addition, the temperature variations observed here are relatively mild when compared to their nuclei mode impact determined by Equation 30.

To say that temperature is not an adequate test metric is not completely true. In a global variable sense this may be true as Figure 55 suggests. However, as mentioned earlier the spreading out of exhaust variables is left to the nature of the plume. In this way a given temperature will bind $\mathrm{CO}_{2}$ to some range. In turn $\mathrm{CO}_{2}$ will bind aerosol concentration. Therefore, direct or indirect temperature can be a valuable test metric in plume prediction of aerosol expansion characteristics. Nevertheless, $\mathrm{CO}_{2}$ appears to have 
a stronger spatial connection to aerosol concentration. As such, the extension to aerosol size distribution will be made and $\mathrm{CO}_{2}$ will be used as a test metric throughout the remainder of this text. More viable physically connected test metrics may exist but will require attention from further plume studies.

It is important to mention that connectivity arguments were made through exhaust map comparisons held to a given axial location. These give no indication of the cumulative axial contribution to dissimilarity among the various exhaust dimensions. In spite of low axial resolution, exhaust variables were found to experience dissimilarity in both a crosssectional and axial sense. To display this dissimilarity, Figure 56 provides a constant dilution surface painted by the temperatures found on it.

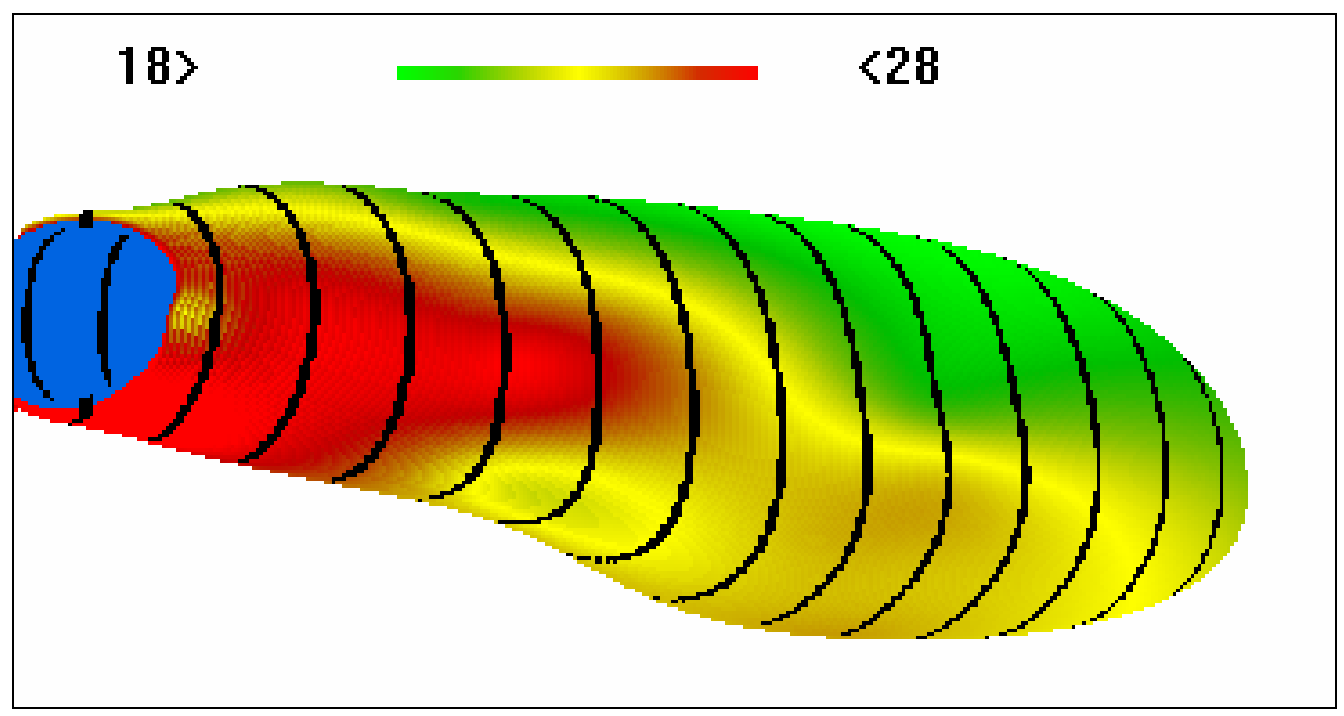

Figure 56 Example Constant Dilution Surface Painted by Temperature. (Color scale refers to temperature (C).)

As can be seen in Figure 56, axial disagreement in the contours of $\mathrm{CO}_{2}$ and temperature far exceed any cross-sectional variation. However, these variations require far more axial resolution to make any connectivity comparisons to aerosol evolution. Increased axial resolution will be required by future studies to alleviate these uncertainties. 
Dispersion of an exhaust plume is due to many coexisting processes including convection, diffusion, and turbulence. As the plume continues slowing down to the speed of the surrounding ventilation air, continuity will dictate an average radial convection. This process should be significant within this study, as the velocity of the ventilation air was $\sim 40 \mathrm{ft} / \mathrm{min}$ while central plume velocities neared $300 \mathrm{ft} / \mathrm{min}$ as far as $10 \mathrm{feet}$ downstream of the exhaust source. This indicates a massive potential for the plume to slow down and as a result spread out.

Diffusion is also responsible for plume dispersion. However, as noted in [19] laminar diffusion is negligible when compared to turbulent diffusion and a plume dispersing under laminar conditions will retain its identity far downstream of the source. Turbulence encourages steep temperature and concentration gradients within the plume caused by convection due to short lived correlations in the velocity fluctuations. These gradients allow molecular diffusion and conductivity a more continued potential. However, being that turbulence originates as a convective process, it should not bias the dispersion of any particular scalars within the plume (i.e. temperature and $\mathrm{CO}_{2}$ ). These biases exist at the molecular level in the form of conductivity and molecular diffusivity. As such, the separation of temperature and $\mathrm{CO}_{2}$ contours is most likely due to the fact that air has a thermal diffusivity of $\sim 26 e^{-3} \mathrm{~m}^{2} / \mathrm{s}$ while the molecular diffusivity of $\mathrm{CO}_{2}$ is $\sim 1.6 e^{-5} \mathrm{~m}^{2} / \mathrm{s}[20]$. 


\subsection{Aerosol Formation and Development}

\subsubsection{Scaling Aerosol Formation through Dilution}

Measurements of aerosol formation due to dilution can be overshadowed by the displacement of the aerosols to larger volumes. To account for the effect of formation only, aerosol size distributions can be scaled to dilution (i.e. $P M \times D R$ ). Scaling in effect, brings the aerosol back to the same unit volume experienced at unmixed conditions (i.e. $D R=1$ ) and allows formation comparisons to be made between multiple dilution ratios. Figure 57 and Figure 58 demonstrate the differences between absolute and scaled aerosol size distributions respectively, which were taken at an axial placement of $6 \mathrm{ft}$ under R75 operation.

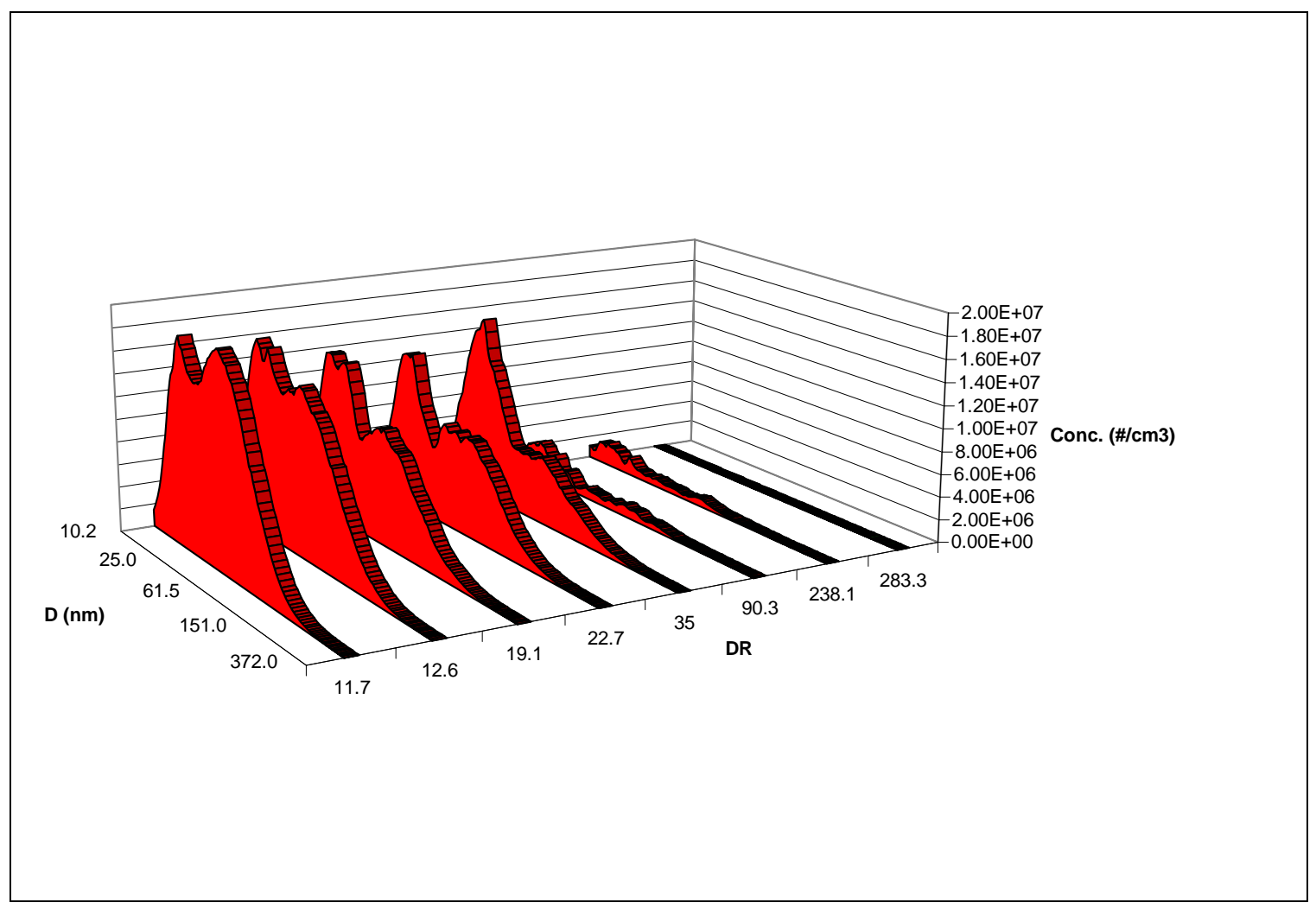

Figure 57 Absolute Aerosol Size Distribution Results at Specified Dilutions. 
As can be seen, Figure 57 suggests a decrease in the absolute aerosol size distribution with increasing dilution. This decrease spans both the nucleation and accumulation modes. The last distribution at $\mathrm{DR}=283.3$ was taken outside of any measureable exhaust plume influence and represents background aerosols. Contrary to the findings of Figure 57, studies such as those performed by Brown et al. [5] suggest that the nucleation mode should increase while the accumulation mode remains relatively unchanged under increasing dilution. Figure 58 shows these same results scaled to dilution.

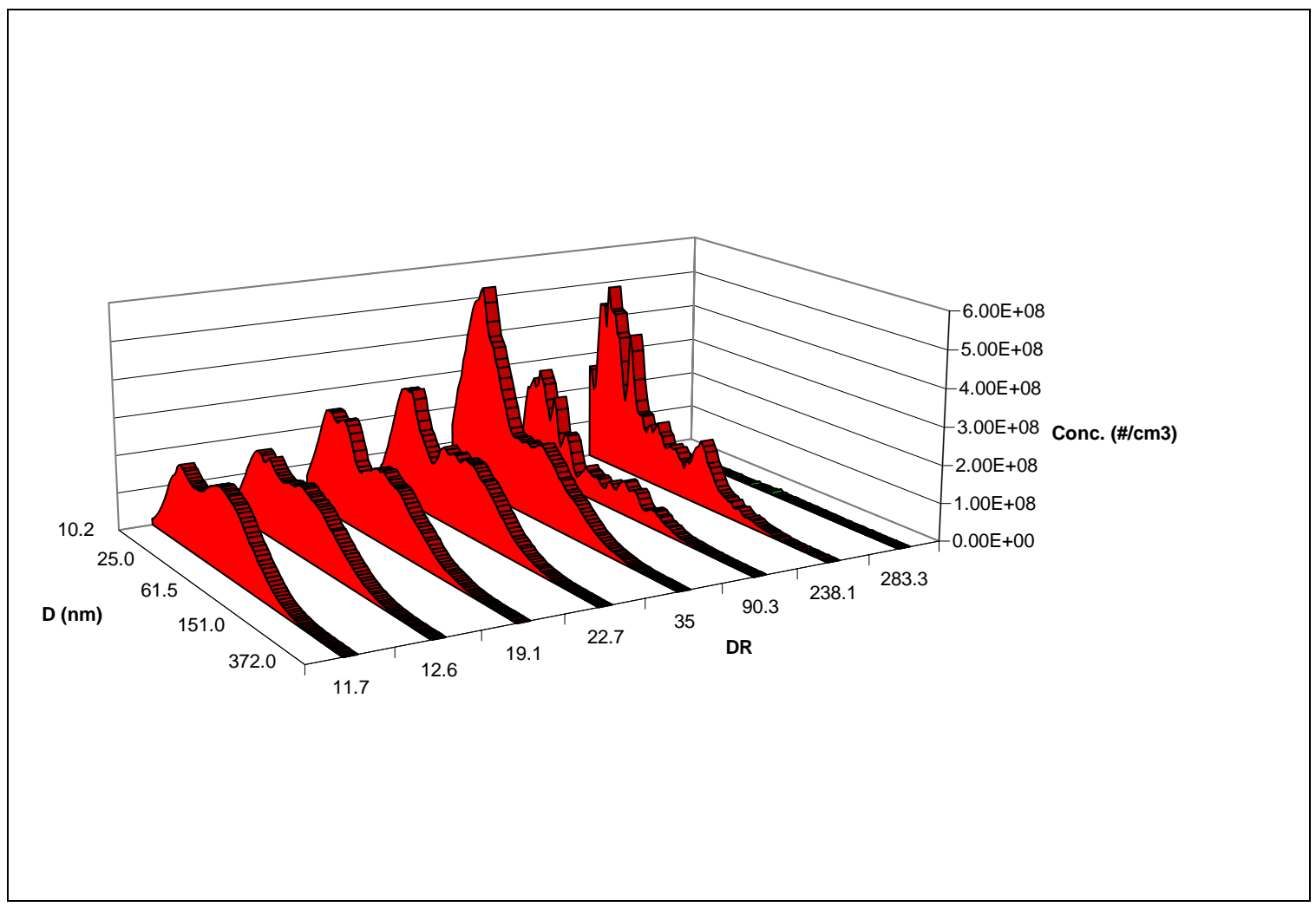

Figure 58 Scaled Aerosol Size Distribution Results at Specified Dilutions.

Figure 58 depicts a trend which appears to be in opposition with that of Figure 57, as it accounts for actual aerosol formation and can be thought of as being independent of sample volume. In addition, this figure appears to be in agreement with accepted ideas 
on aerosol formation under dilution. As such, aerosol size distributions subsequently described will be understood as being scaled to dilution ratio unless otherwise noted.

\subsubsection{Aerosol Mapping Results}

The following sections present the aerosol size distribution results obtained while the engine was operated under R75 and I30 conditions. All data will include an accompanying $\mathrm{CO}_{2} \operatorname{map}(\mathrm{s})$ to demonstrate sample placement within the plume.

\subsubsection{R75}

This section describes the aerosol size distribution results obtained while the engine was operated under R75 conditions. Figure 59 and Figure 60 demonstrate the $\mathrm{CO}_{2}$ map placement and average aerosol distributions respectively for an axial placement of $6 \mathrm{ft}$.

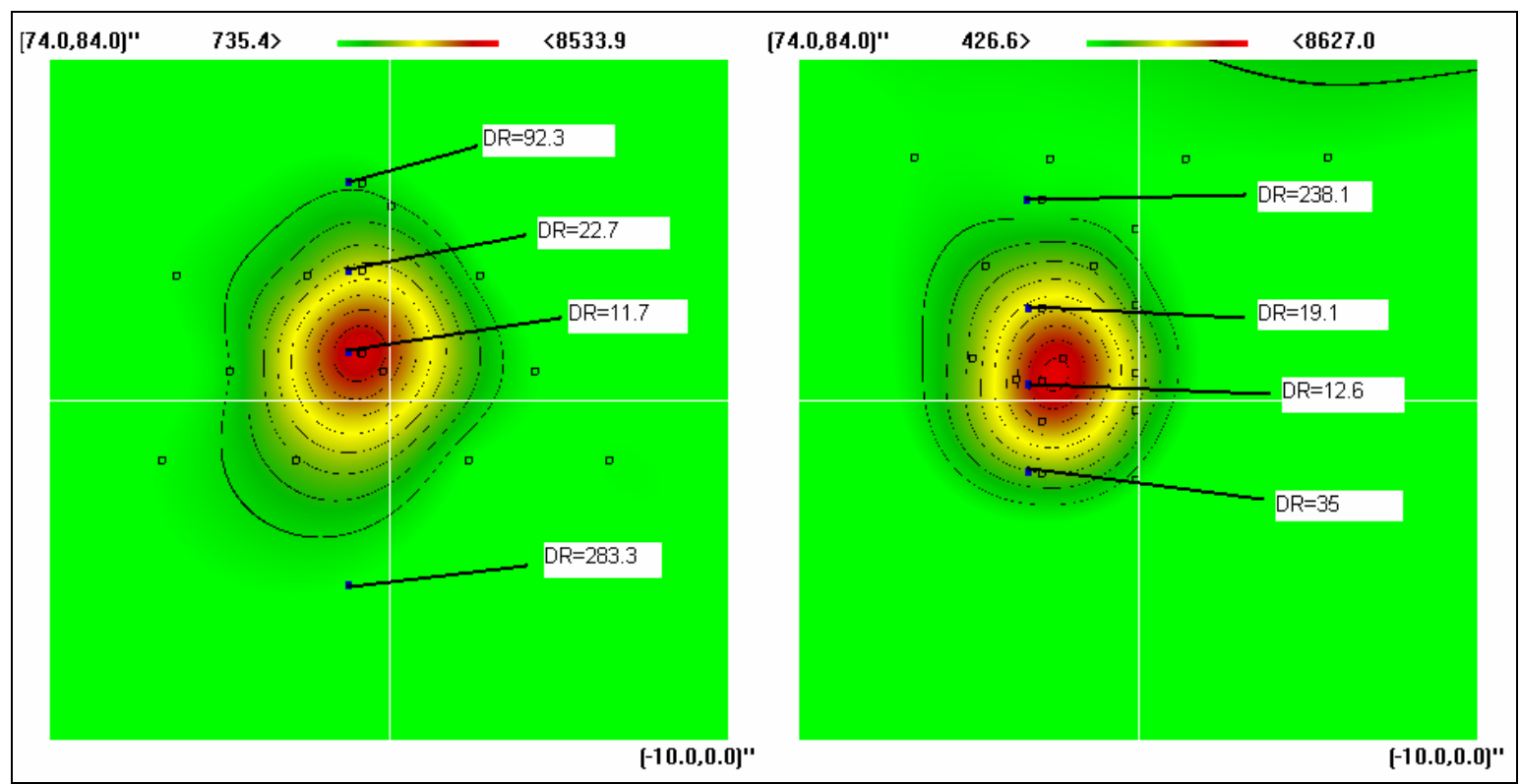

Figure 59 6ft Aerosol Size Distribution Measurement Locations for Figure 60 


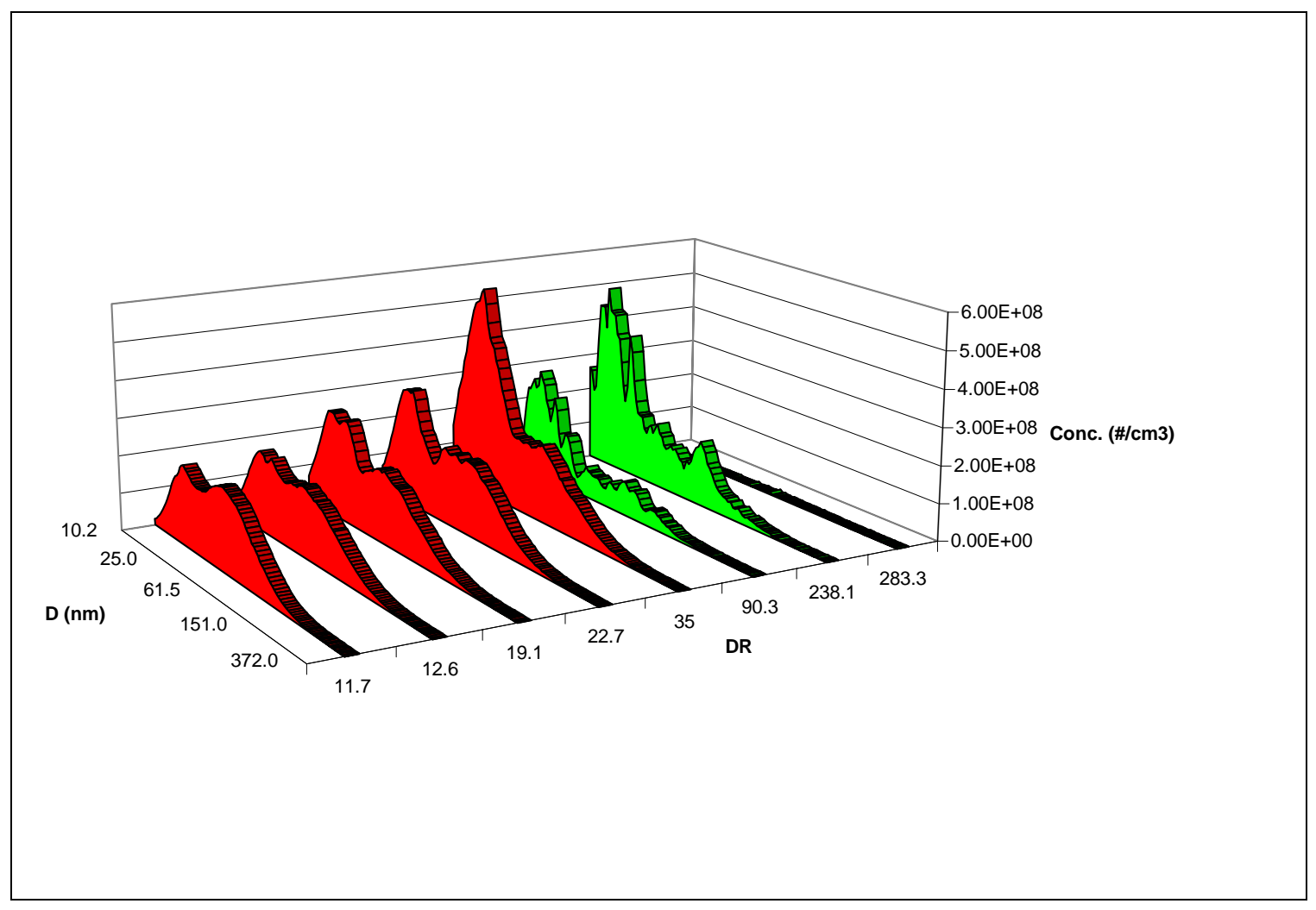

Figure 60 6ft Aerosol Size Distribution Results for Days 5 and 6

As can be seen in Figure 60, increasing dilution results in nucleation mode formation with little change observed in the accumulation mode. This is as observed in Brown et al.'s [5] study. However, the aerosol size distributions recorded beyond a dilution ratio of 90.3 are observed to disobey this general trend. These distributions are thought to be a complex mixture of background aerosols and aerosols formed from actual dilution, but are included regardless to demonstrate this effect. In fact, the distribution observed at a recorded DR of 283.3 appears to be a complete mixture of background aerosols. In addition, the spatial connection of aerosol size distribution to $\mathrm{CO}_{2}$ may break down in regions distant from the plume's center, such as they did with temperature.

Care must be observed when referring to high dilutions. As dilution ratio tends towards infinity, actual plume involvement decreases. In addition, measurement uncertainty 
increases when trying to record dilutions near contaminated background levels. Therefore, blindly scaling aerosol size distributions to DR can result in erroneous observations, which is one reason why the $\mathrm{CO}_{2}$ placement maps are provided along with all aerosol size distribution data within this study.

In addition, the results shown in Figure 60 cannot be said to be the sole cause of physical processes occurring at 6 feet. These observations may be the spatial continuation of processes that occurred at earlier axial locations. Further axial resolution of this region will be required by future studies to separate spatial and physical occurrences.

Figure 61 and Figure 62 provide the $\mathrm{CO}_{2}$ placement map and aerosol size distribution data respectively for the $10 \mathrm{ft}$ axial station.

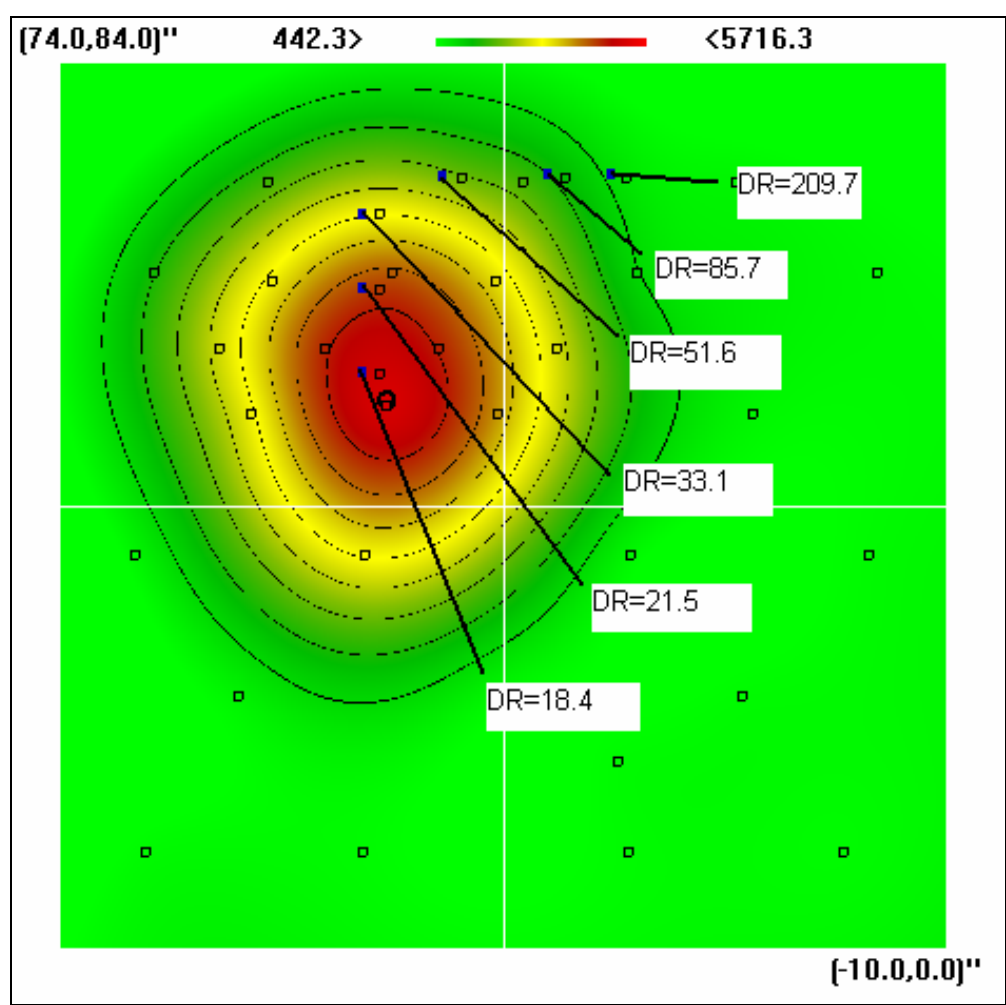

Figure $6110 \mathrm{ft}$ Aerosol Size Distribution Measurement Locations for Figure 62 


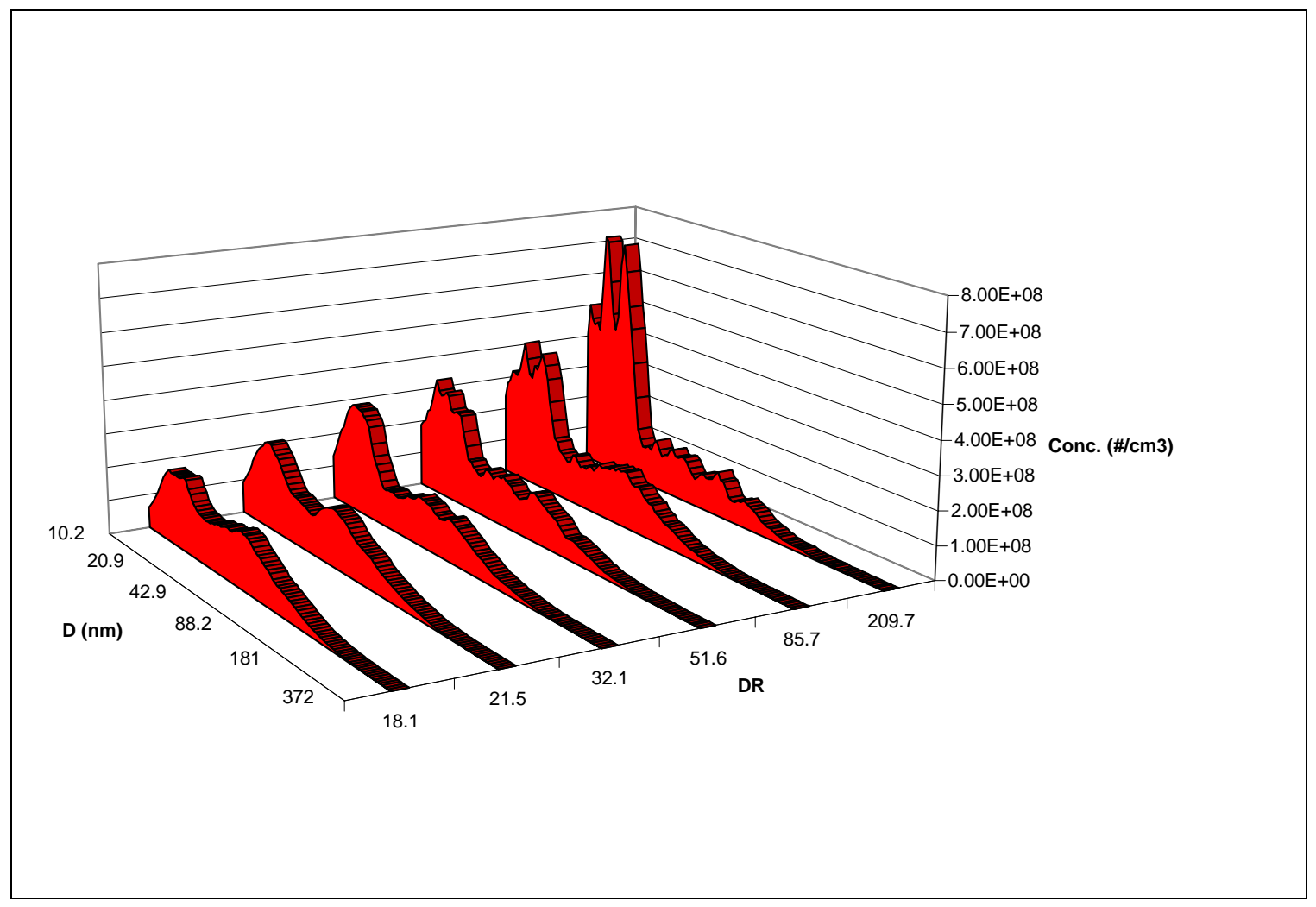

Figure 62 10ft Aerosol Size Distribution Results for Day 7.

The trends observed in Figure 62 are similar to those found in Figure 60. However, the upper limit of the aerosol size distributions at 10 feet are much higher than those observed at 6 feet. This increase is an indication that aerosol formation occurs beyond distances of 6 feet under R75 operation. In addition, the map placement locations are found over a longer curved path through the $\mathrm{CO}_{2}$ field. This approach increased the likelihood of discovering discrepancies in the spatial connection of $\mathrm{CO}_{2}$ to aerosol size distribution. The path also extends through regions which were found to have large dissimilarities in the expansion patterns of $\mathrm{CO}_{2}$ and temperature.

Figure 63 and Figure 64 provide the $\mathrm{CO}_{2}$ placement maps and aerosol size distribution data respectively for the 20 foot axial station. 


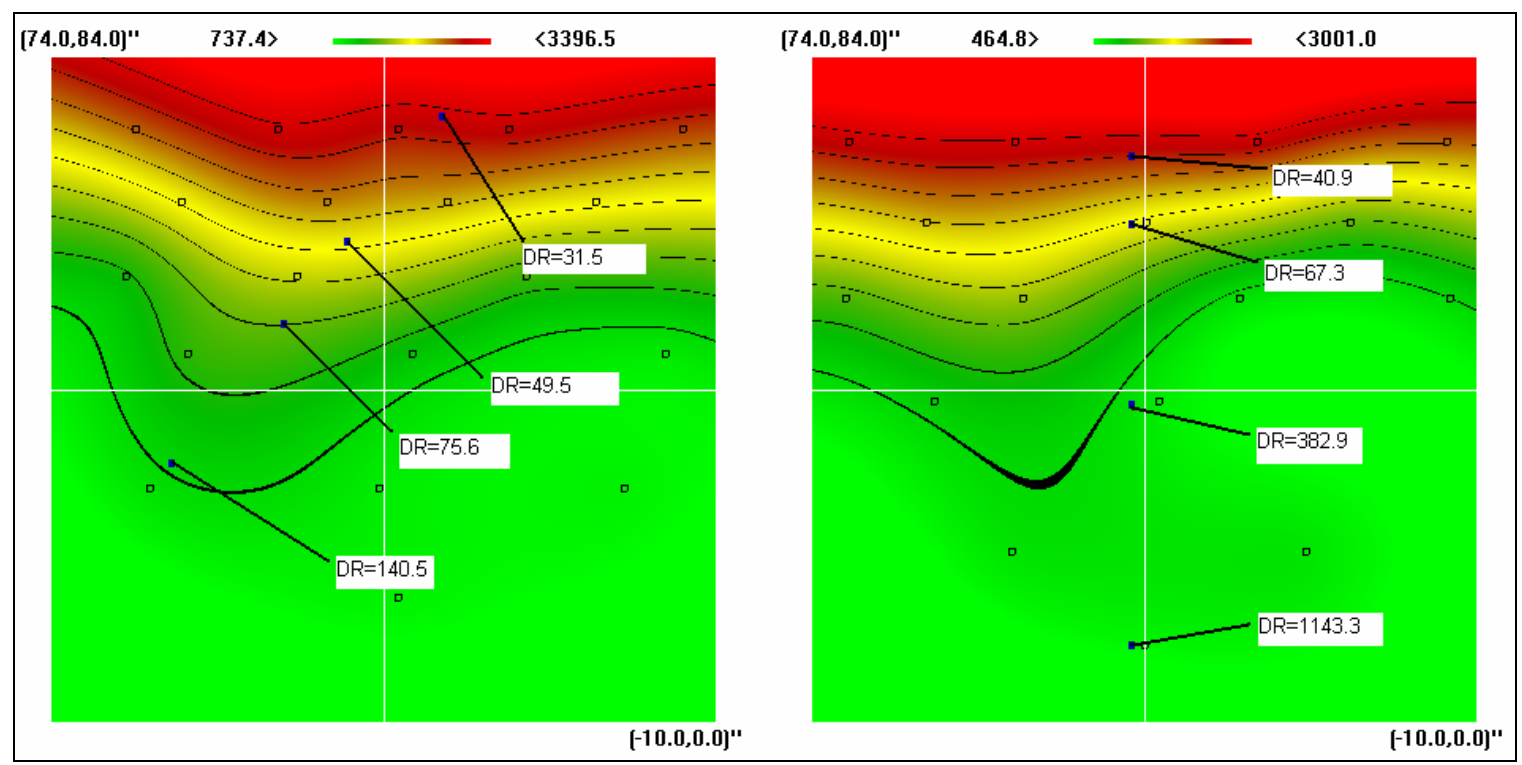

Figure $6320 \mathrm{ft}$ Aerosol Size Distribution Measurement Locations for Figure 64

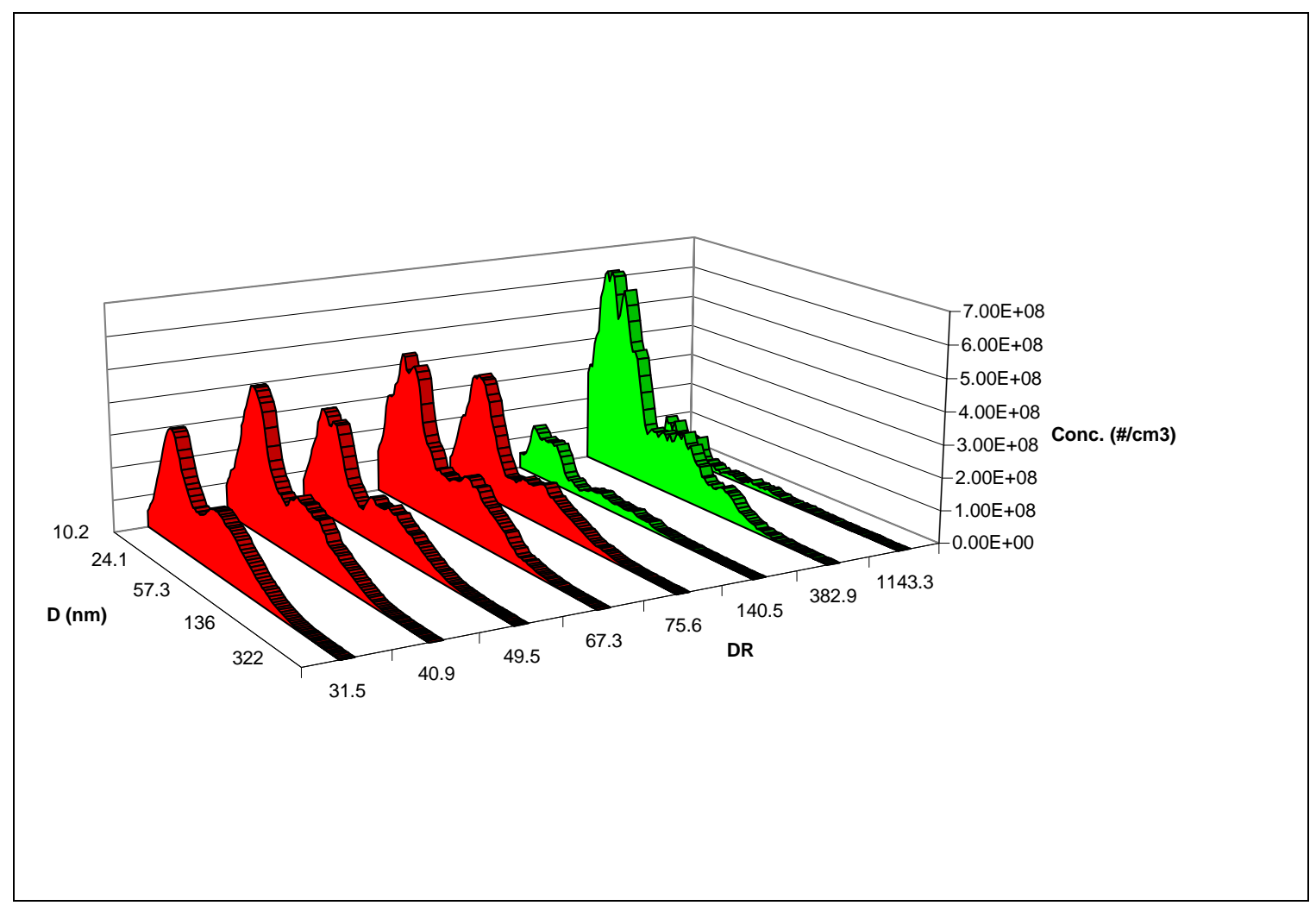

Figure 64 20ft Aerosol Size Distribution Results 
The aerosol size distribution results well within the plume of Figure 64, appear to be uninfluenced by dilution, which is a strong indication that aerosol formation has ceased within 10 to 20 feet of the exhaust outlet. In fact, the upper limit of the distributions has decreased significantly from 10 feet suggesting that aerosol losses and / or conversions are occurring within the plume. The variation in the last three size distributions can be described as uncertainty in calculated dilution ratios.

Figure 65 and Figure 66 provide the $\mathrm{CO}_{2}$ placement map and aerosol size distribution data respectively for the 50 foot axial station. 


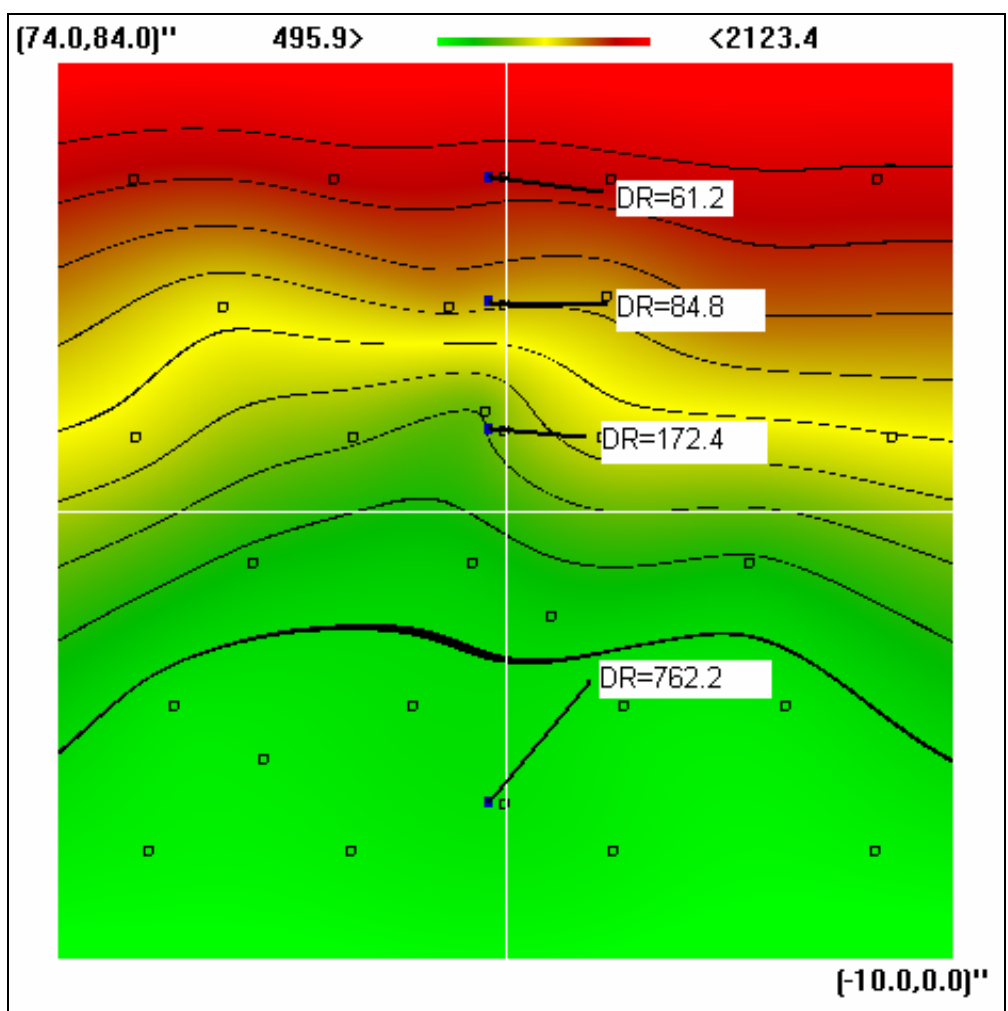

Figure $6550 \mathrm{ft}$ Aerosol Size Distribution Measurement Locations for Figure 66

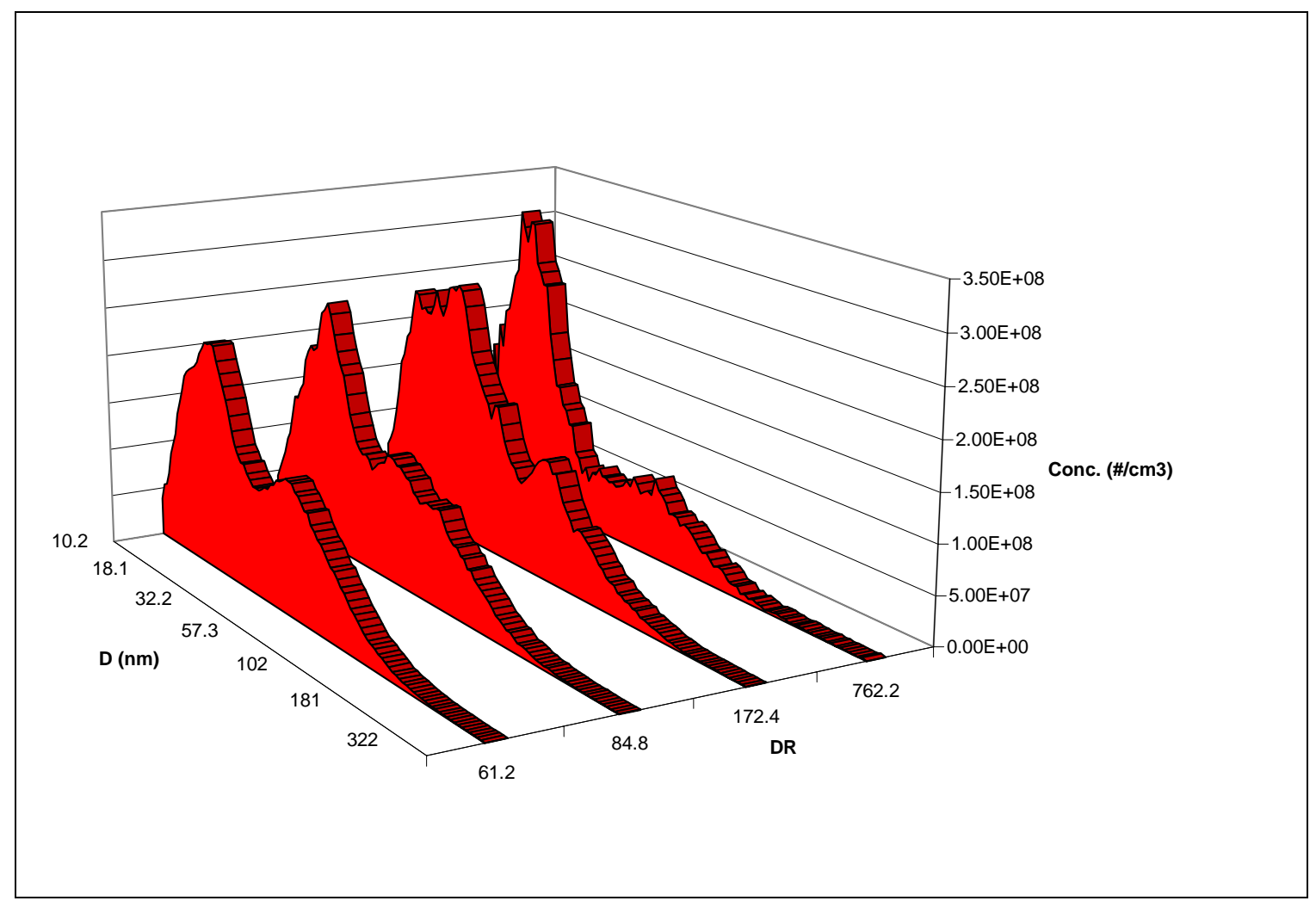

Figure 66 50ft Aerosol Size Distribution Results 
As in Figure 64, the aerosol size distribution results of Figure 66 appear invariant with

dilution ratio. In addition, peak distribution values have diminished slightly suggesting a continued particle loss from 20 feet.

Figure 67 and Figure 68 provide the $\mathrm{CO}_{2}$ placement maps and aerosol size distribution data respectively for the 125 foot axial station. 


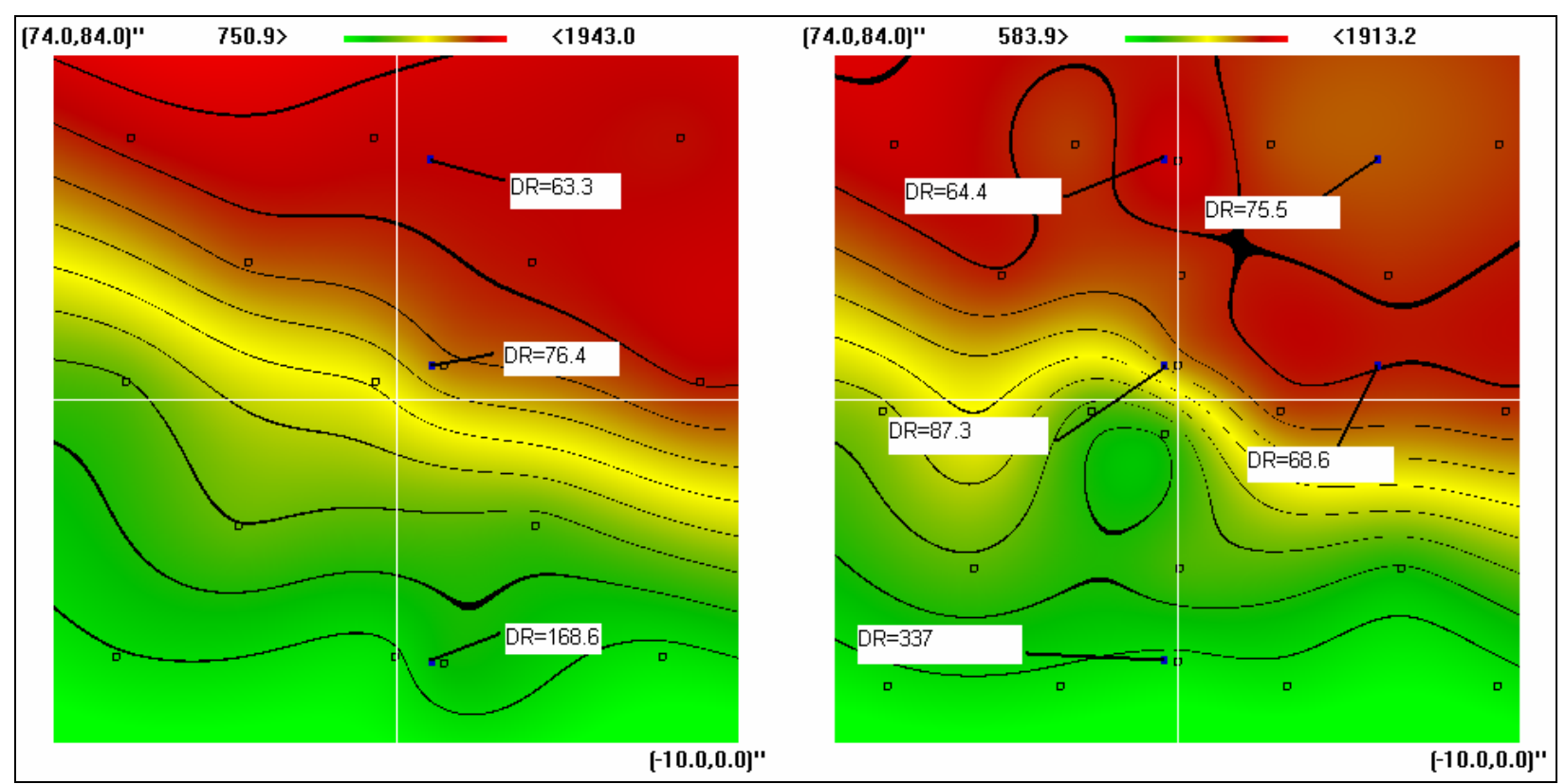

Figure 67 125ft Aerosol Size Distribution Measurement Locations for Figure 68

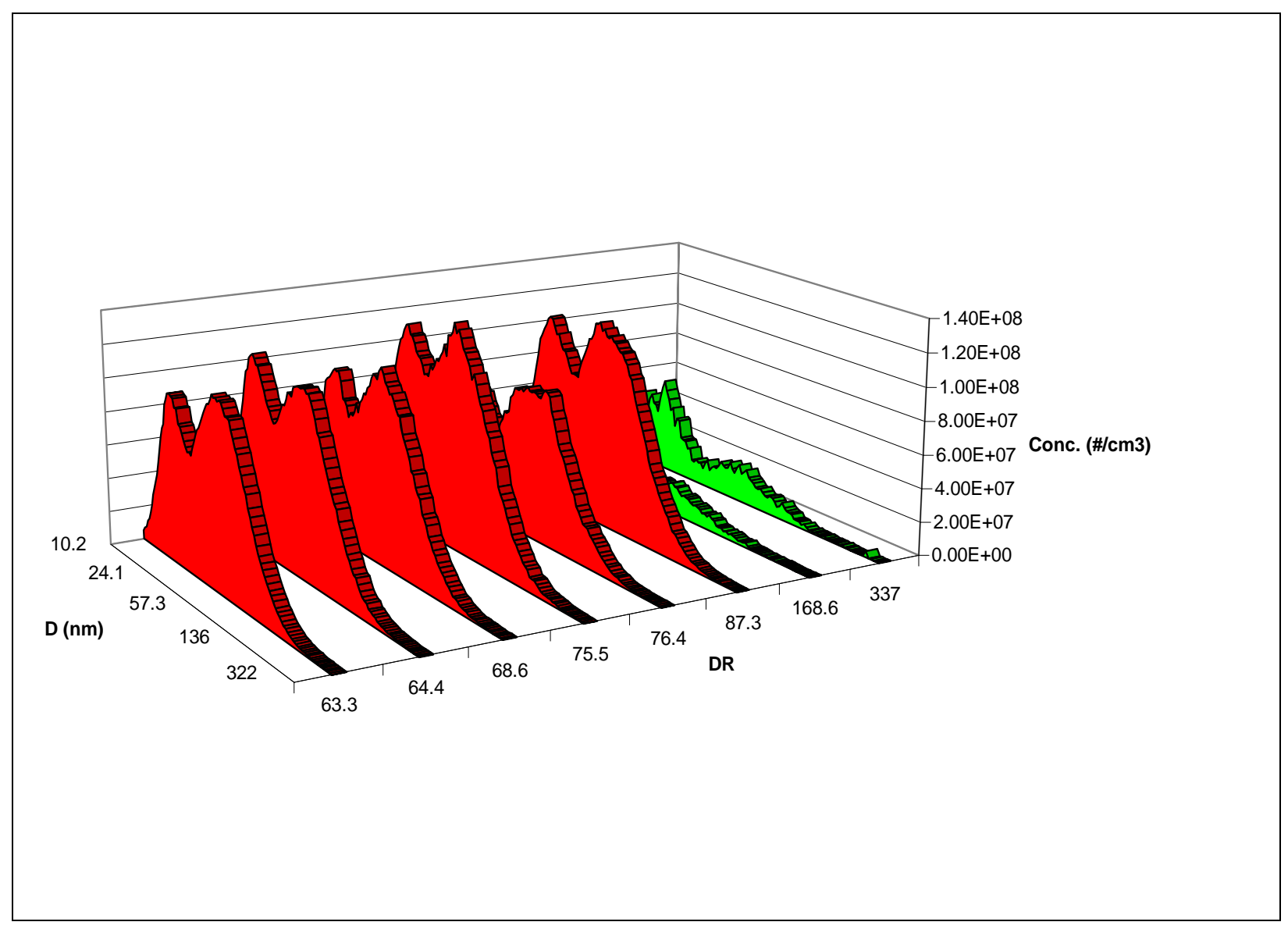

Figure 68 125ft Aerosol Size Distribution Results 
Figure 68 suggests further particle loss from the 20 foot station nearing one order of magnitude. In addition, size distributions well within the plume are still invariant with dilution ratio.

\subsubsection{R75 (Fine Resolution)}

This section is devoted towards the fine cross-sectional mapping of aerosol size distribution data under R75 operation. Aerosol size distributions will be provided for each of the $\mathrm{CO}_{2}$ contour bands mapped at 1,3 , and 10 foot axial placements. These distances were found in the previous section to involve aerosol formation.

The first set of aerosol data was taken on day 13 at an axial placement of 1 foot. As mentioned earlier, the $\mathrm{CO}_{2}$ analyzer is incapable of accurately mapping this region in its entirety by any single channel. As such, a blend of channels 1 and 2, based on Figure 69, was used to calculate dilution ratios for this region.

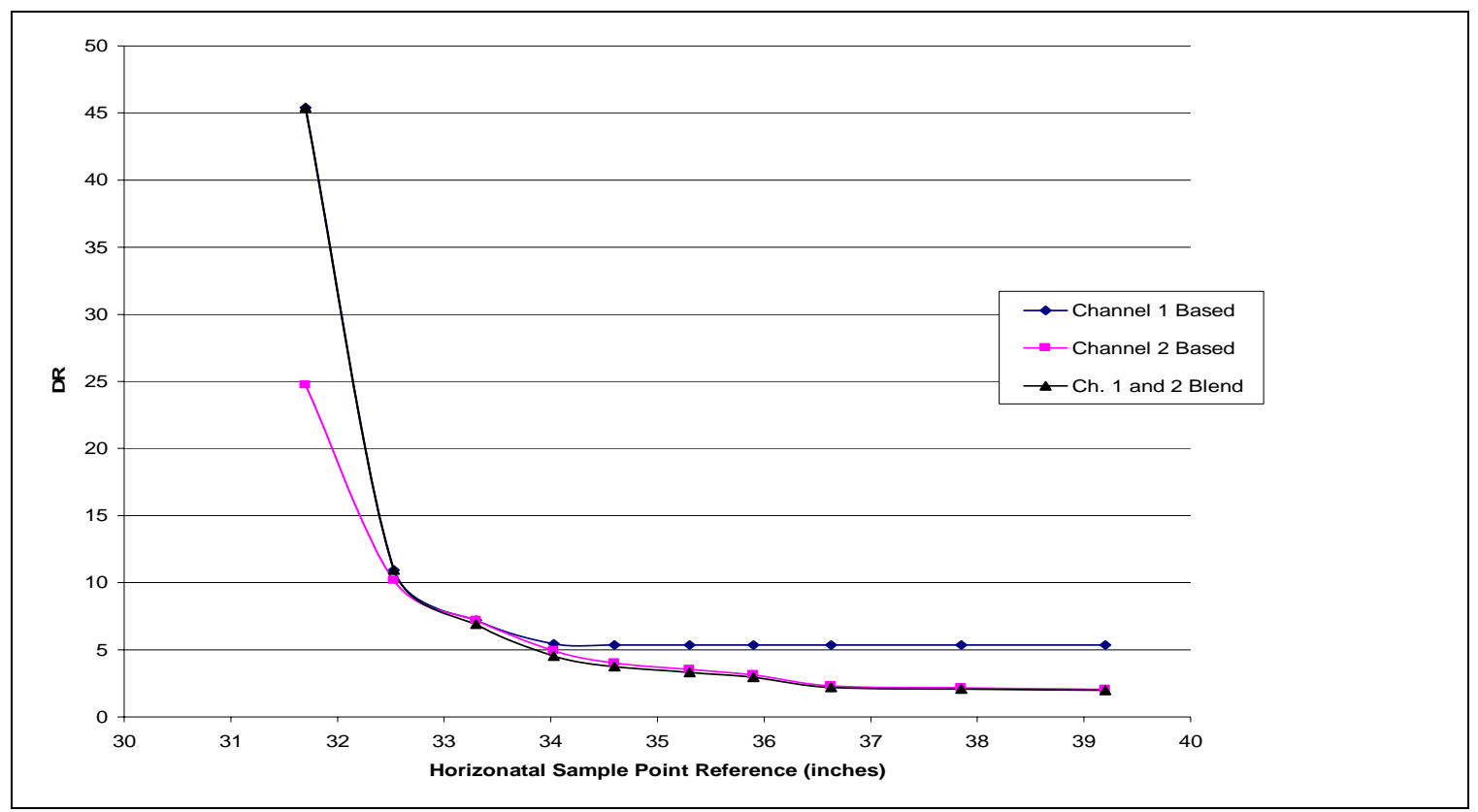

Figure 69 1ft DR Channel Comparison and Selected Blend 
Figure 70 and Figure 71 show the resulting sample placement map and aerosol size distributions respectively for this 1 foot axial station.

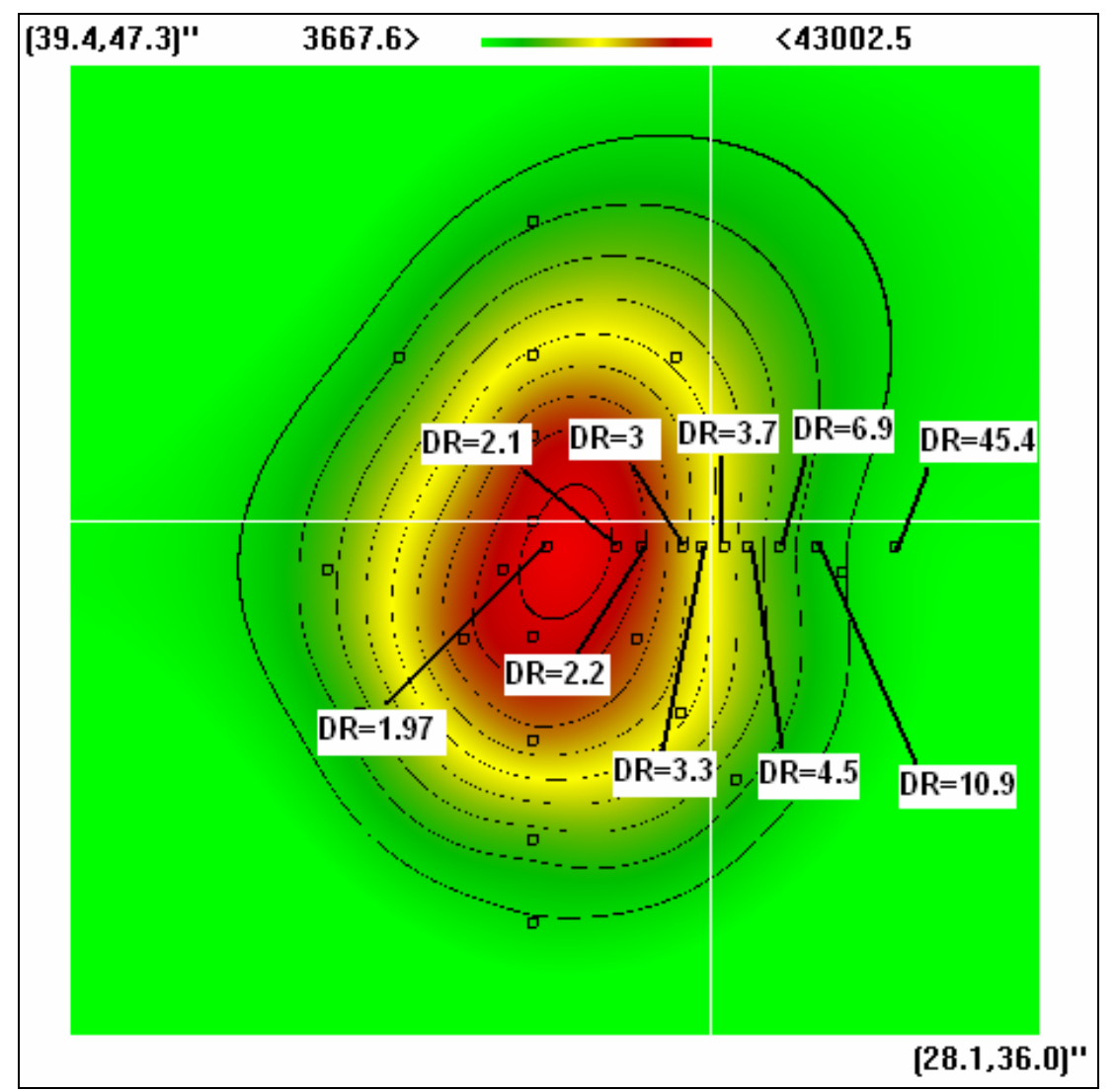

Figure 70 1ft Aerosol Size Distribution Measurement Locations for Figure 71 


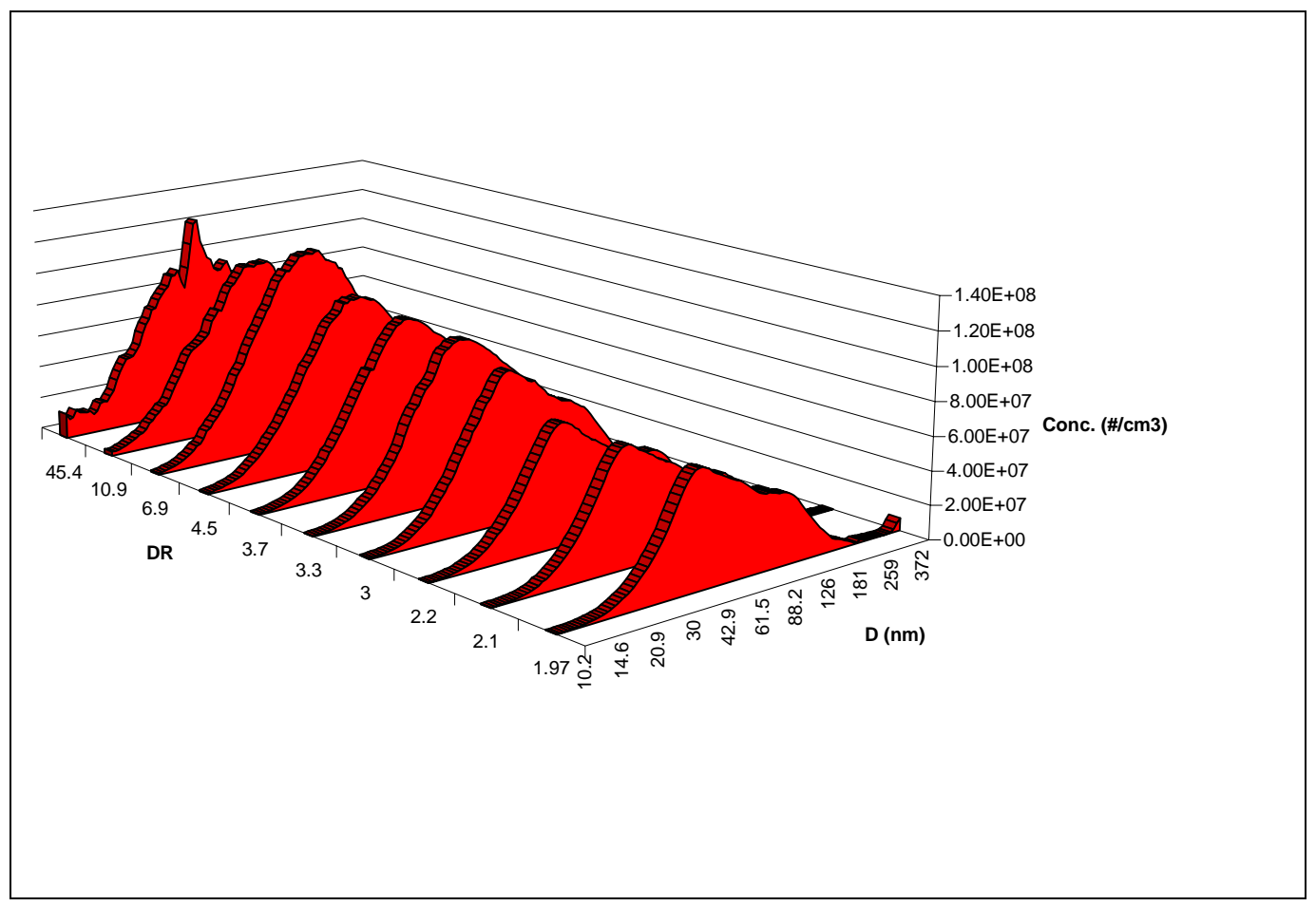

Figure 71 1ft Aerosol Size Distribution Results

As can be seen in Figure 71, aerosol formation at an axial placement of 1 foot is relatively stagnant. Only at the very edge of the plume under a dilution ratio of $\sim 45$ does the nuclei mode appear to develop. Such high dilution ratios observed at other more distant axial stations were shown to involve the nuclei mode. As such, it is suspected that the resident times of this station are not long enough to facilitate particle formation.

Figure 72 and Figure 73 show the sample placement map and aerosol size distributions respectively for the 3 foot axial station taken on day 13 . 


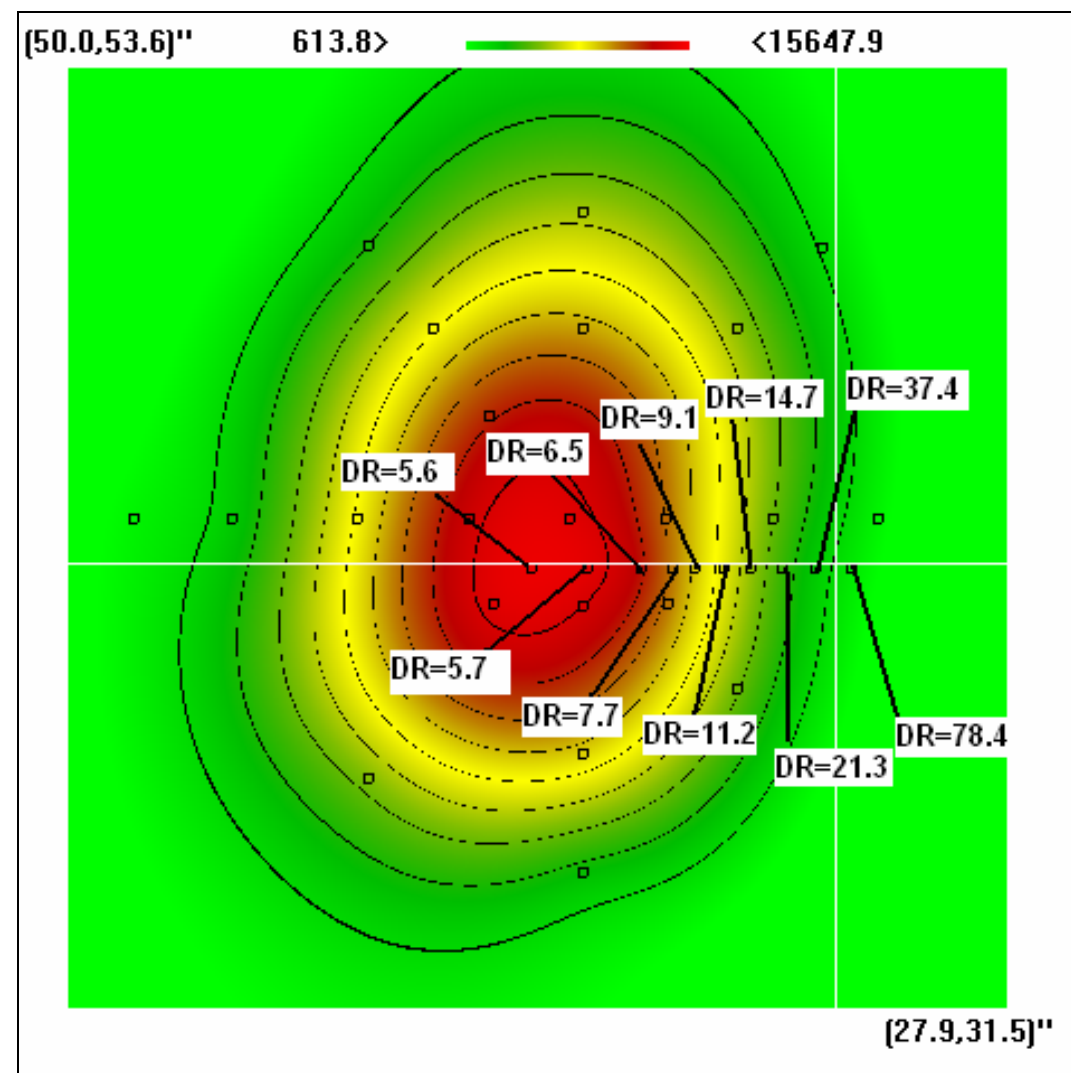

Figure 72 3ft Aerosol Size Distribution Measurement Locations for Figure 73

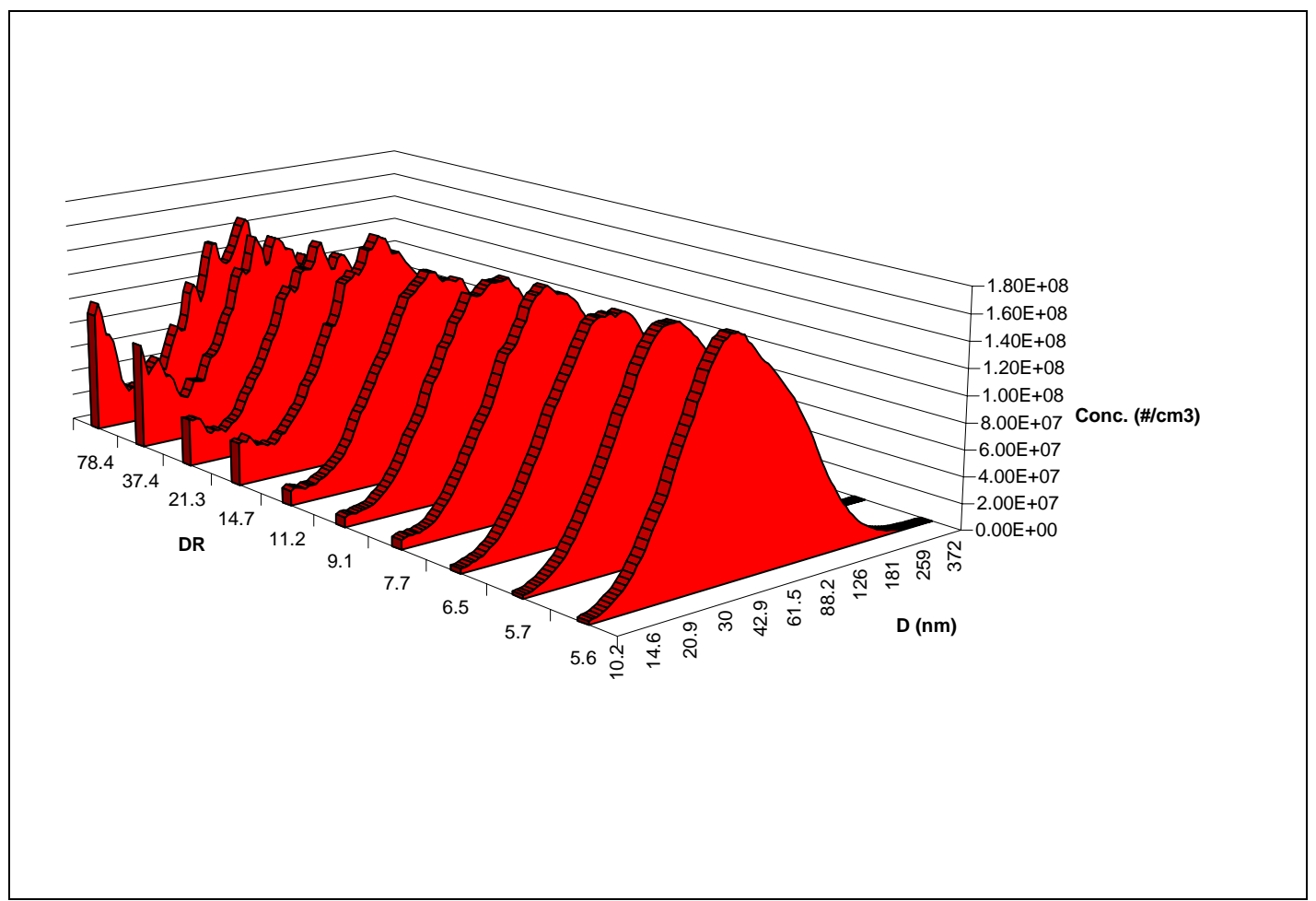

Figure 73 3ft Aerosol Size Distribution Results 
As can be seen in Figure 73, the nuclei mode of this data set occurs at dilution ratios much lower than those required at the 1 foot station. This observation is an indication of a resident dependence of the nuclei mode. In addition, the nuclei mode appears to extend below the lower cut off point of the instrumentation, which was not apparent for the previous section and suggests a shift in environmental conditions. With no major differences in the conditions recorded in Table 7, variations in the sulfur ( 300 ppm) content of the fuel may have caused this observance.

Figure 74 and Figure 75 show the sample placement map and aerosol size distributions respectively for the 10 foot axial station taken on day 12 .

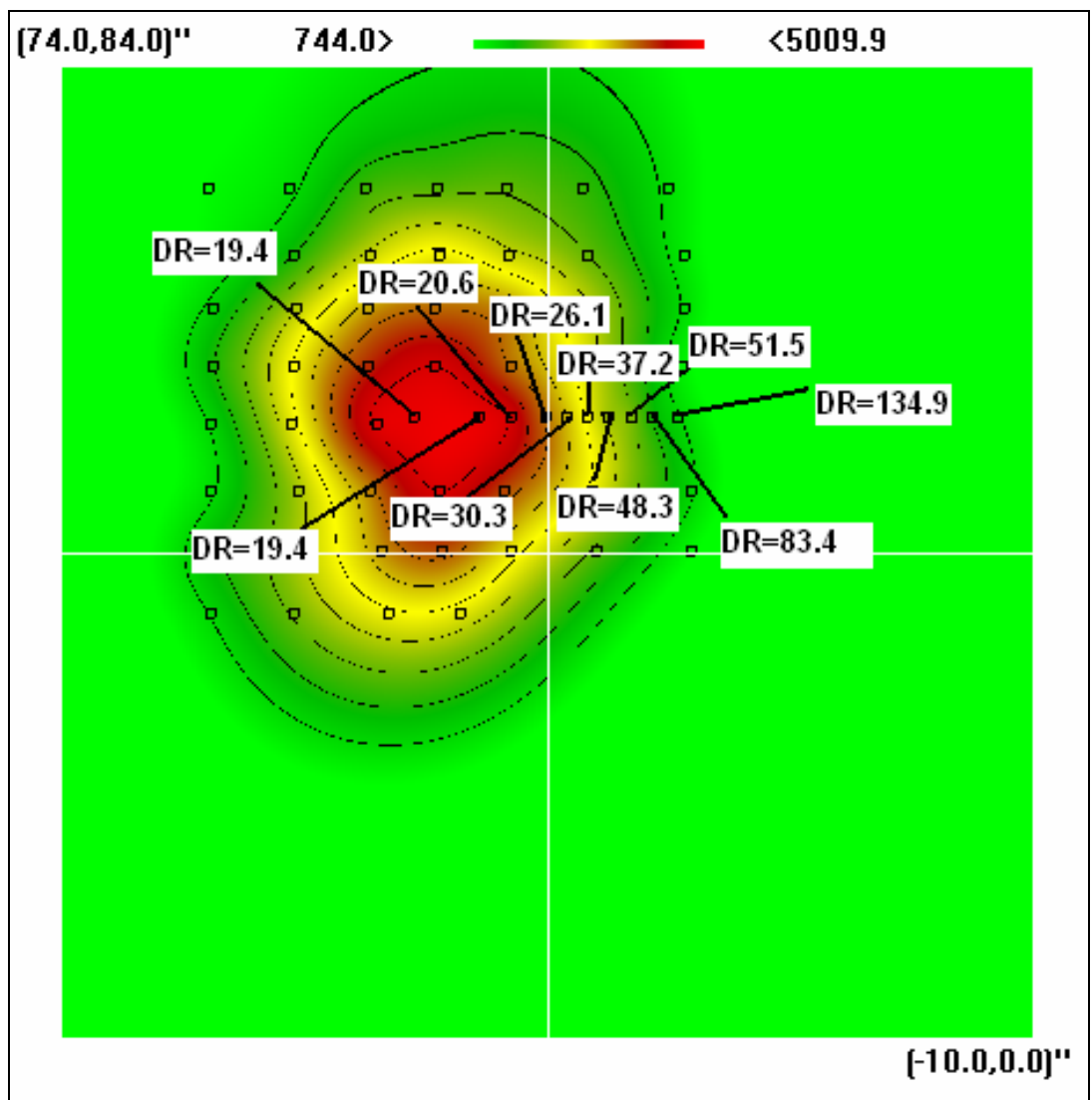

Figure 74 10ft Aerosol Size Distribution Measurement Locations for Figure 75 


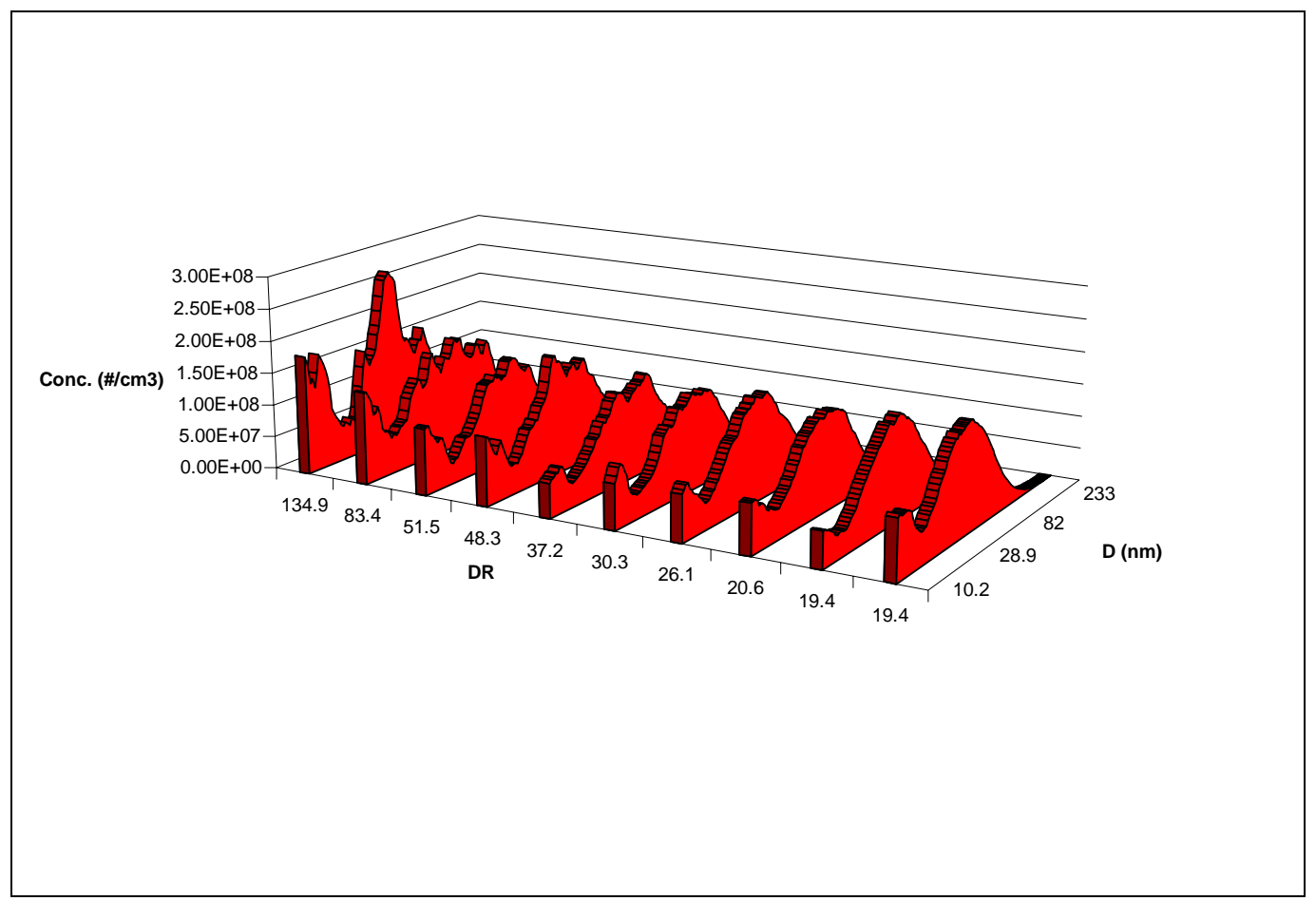

Figure 75 10ft Aerosol Size Distribution Results.

As can be seen in Figure 75, at 10 feet the nuclei mode is present throughout the entire plume. In addition, nuclei mode variations are irregularly minor for dilution ratios up to 37. This invariance might indicate an overwhelming dominance of residence over dilution ratio in the formation of particles. As such, ever larger dilution ratios are required to differentiate these distributions.

\subsubsection{I30}

This section describes the aerosol size distribution results acquired under I30 operation. Figure 76 and Figure 77 provide the $\mathrm{CO}_{2}$ map placement and aerosol size distribution results respectively taken on day 10 at an axial placement of 1 foot. 


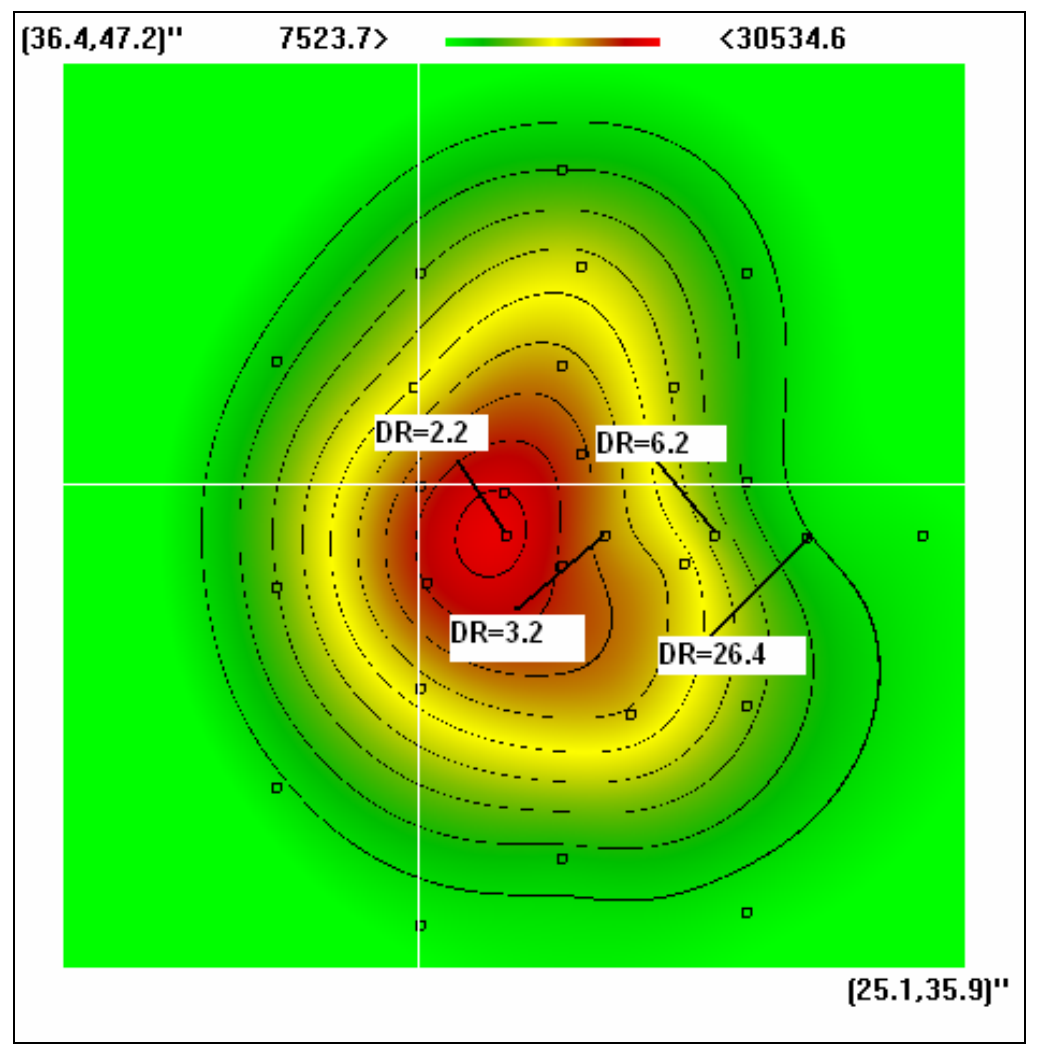

Figure $761 \mathrm{ft}$ Aerosol Size Distribution Measurement Locations for Figure 77

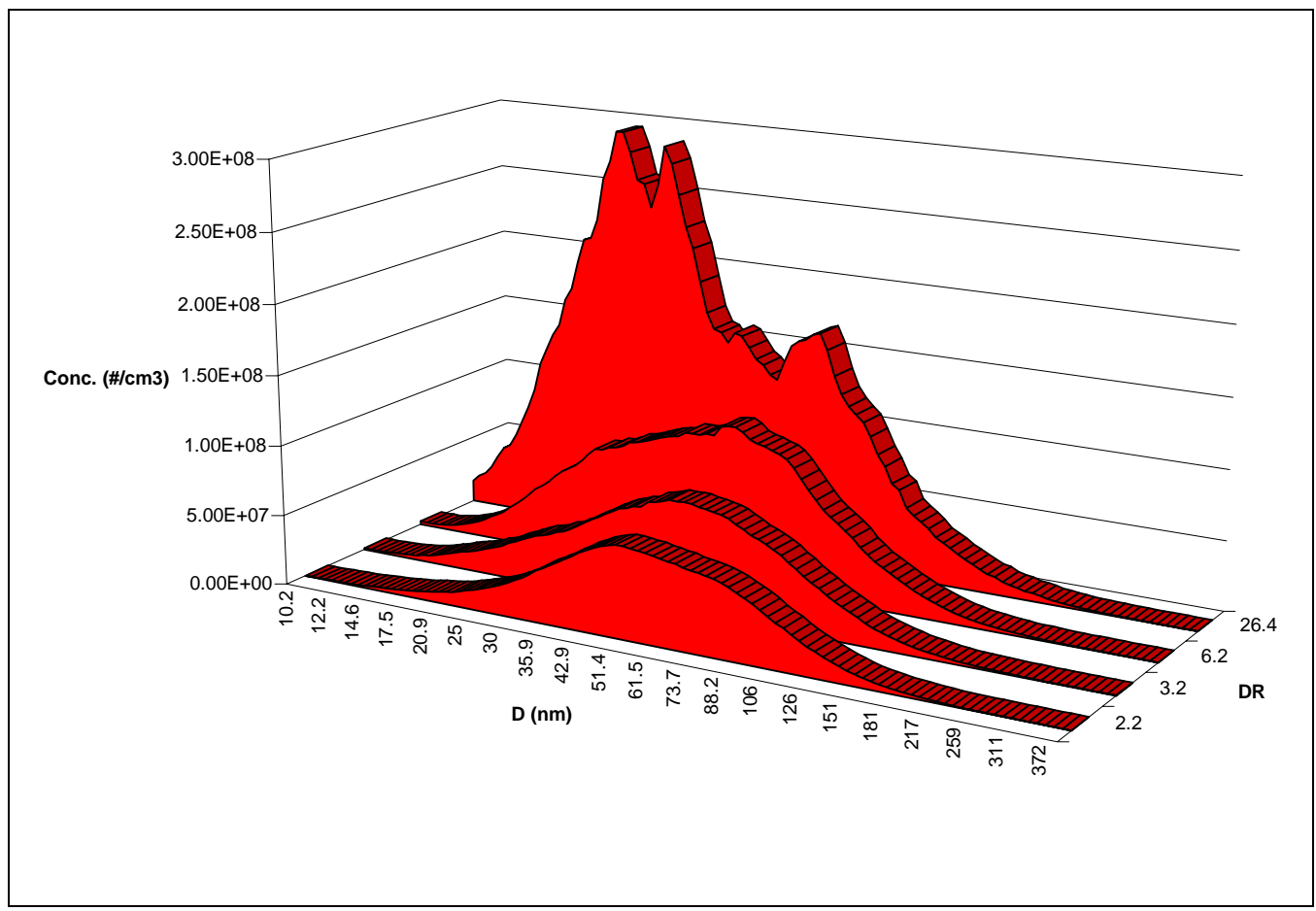

Figure 77 1ft Aerosol Size Distribution Results. 
As can be seen in Figure 77, the aerosol size distributions of the 1 foot station appear to move slightly towards smaller particle diameters and larger particle numbers with increasing dilution. This observation may be caused by a break up in the accumulation mode particles due to the agitation present in more diluted regions. In addition, no distinct nuclei mode appears present in any of the distributions.

Figure 78 and Figure 79 provide the $\mathrm{CO}_{2}$ map placement and aerosol size distribution results respectively taken on day 8 at an axial placement of 6 foot.

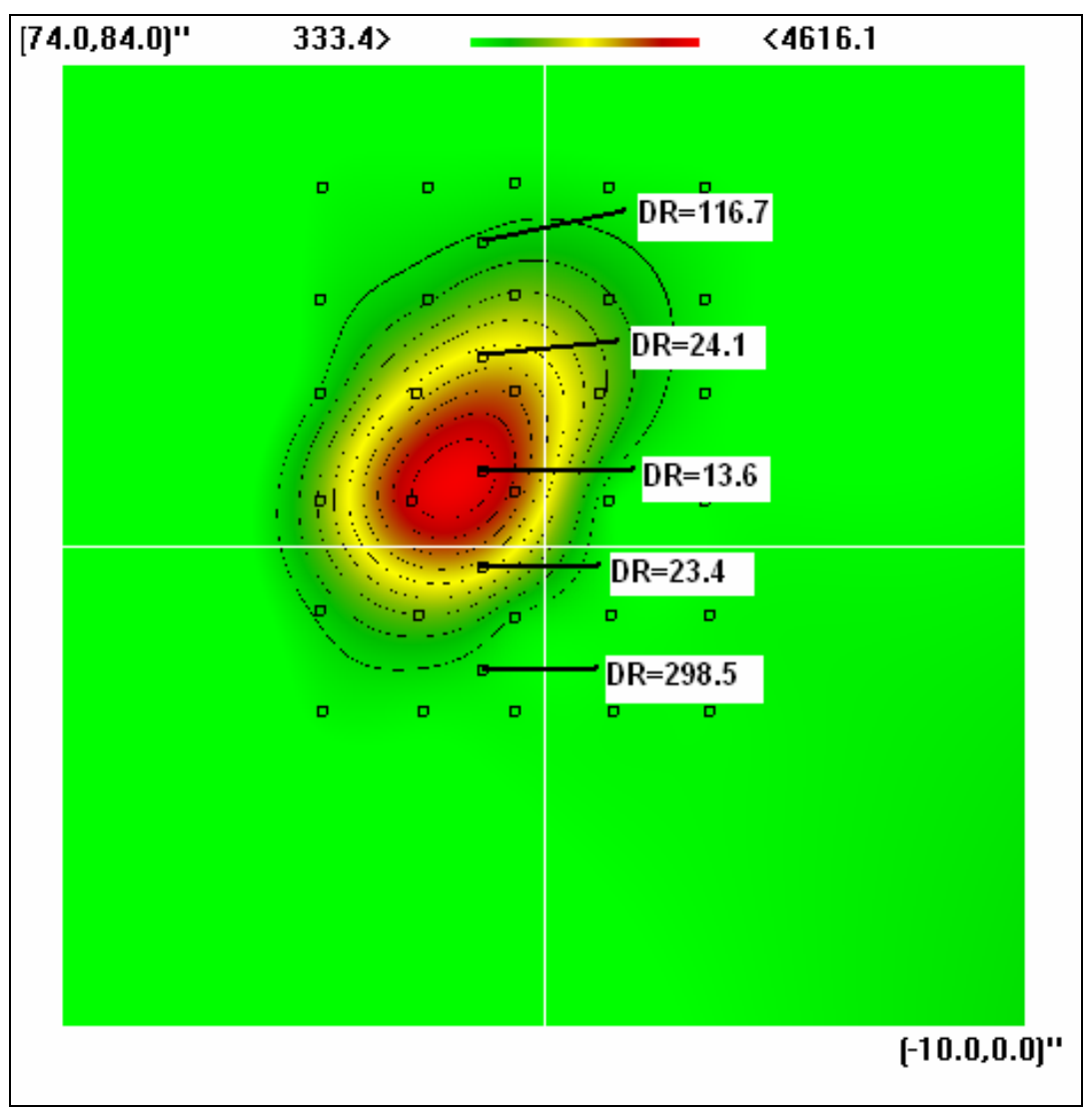

Figure 78 6ft Aerosol Size Distribution Measurement Locations for Figure 79 


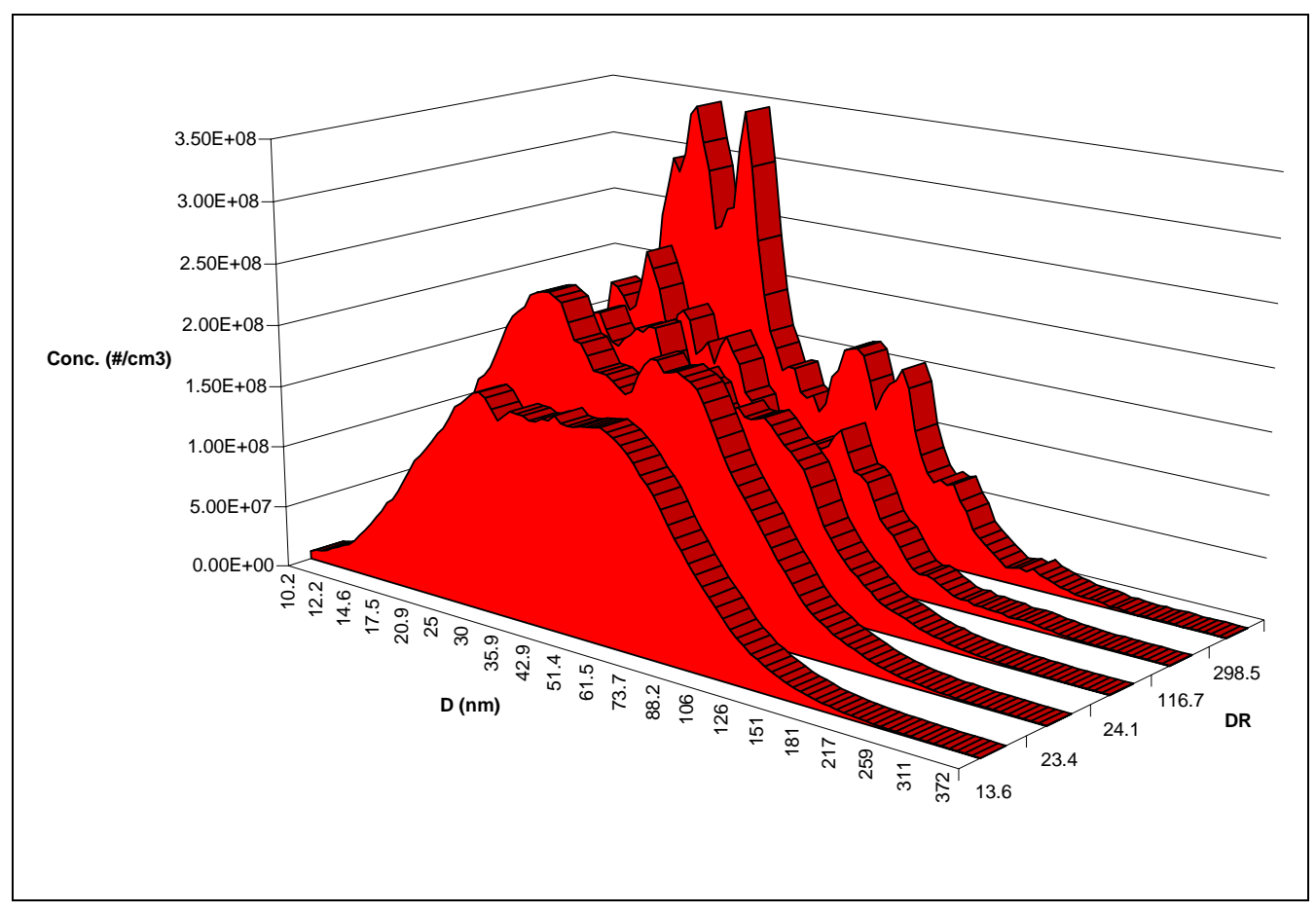

Figure 79 6ft Aerosol Size Distribution Results

The aerosol size distributions of Figure 79 appear invariant across the plume. In addition, the midpoint of each distribution is in the $30 \mathrm{~nm}$ range. This midpoint is significantly lower than largest midpoint observed at the 1 foot station suggesting a break up of the accumulation mode.

Figure 80 and Figure 81 provide the $\mathrm{CO}_{2}$ map placement and aerosol size distribution results respectively taken on day 8 at an axial placement of 10 foot. 


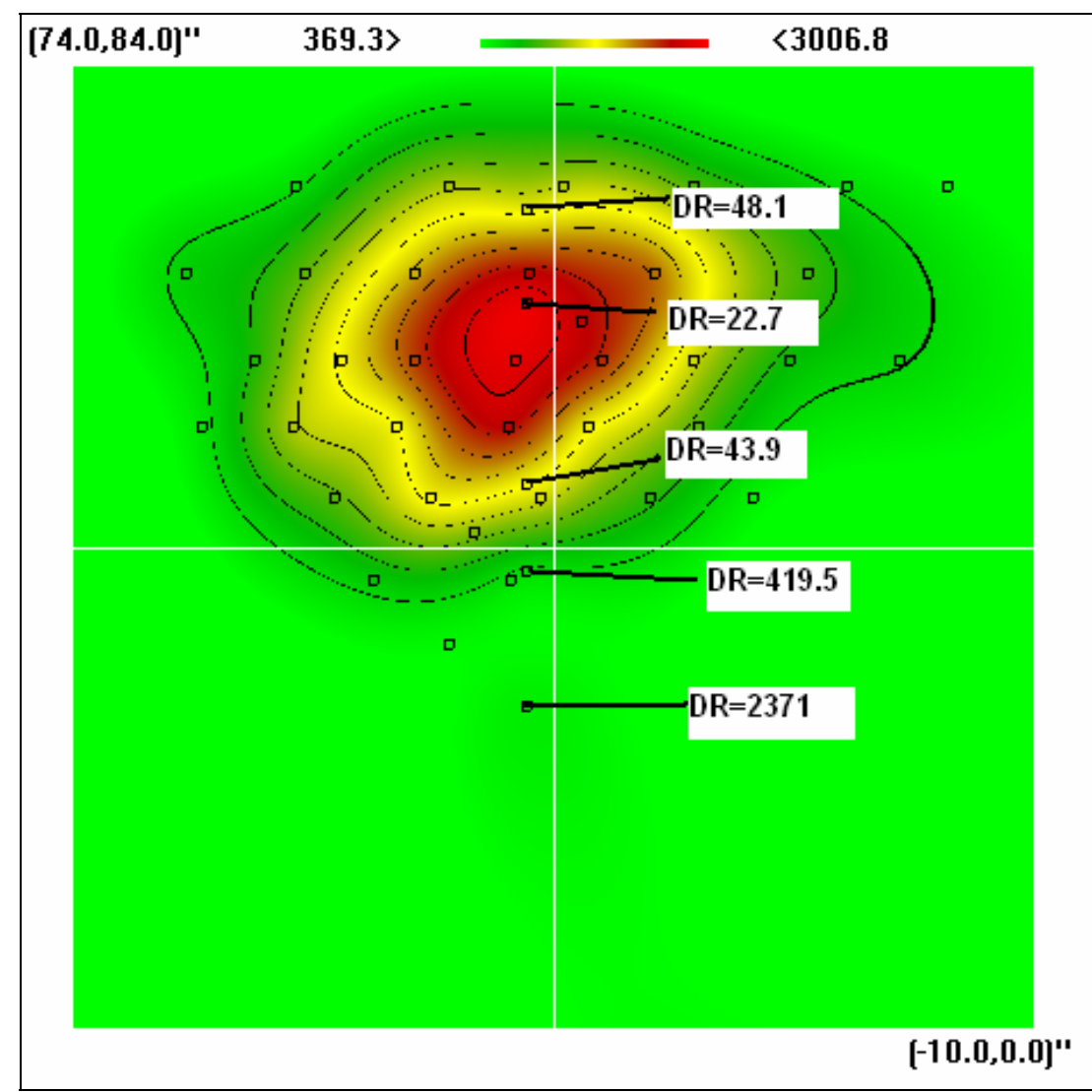

Figure 80 10ft Aerosol Size Distribution Measurement Locations for Figure 81

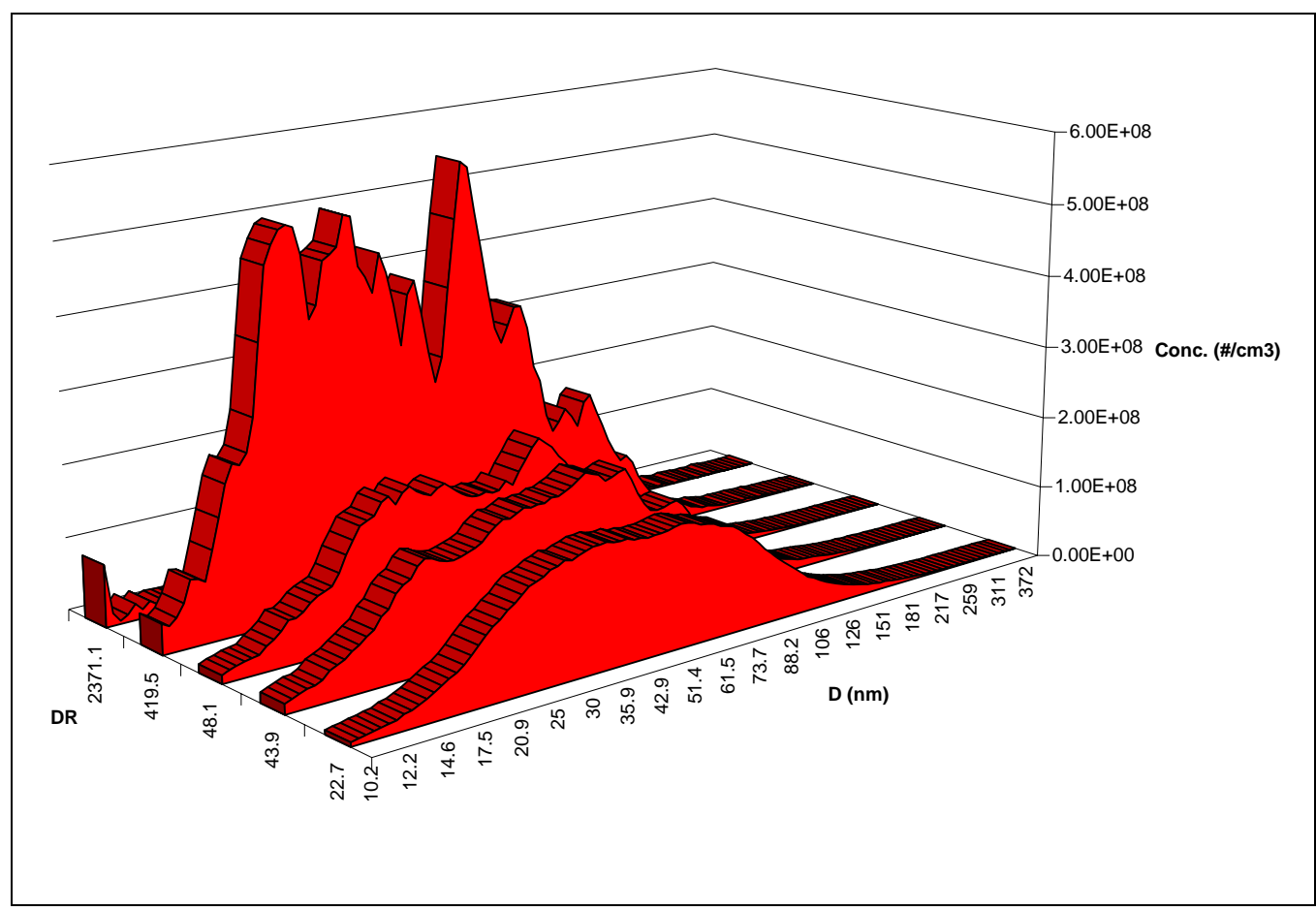

Figure 81 10ft Aerosol Size Distribution Results. 
The aerosol size distributions of Figure 81 appear invariant up to a dilution ratio of $\sim 48$ with no apparent nuclei mode. The distribution observed at a dilution ratio of 419.5 could be misrepresented by errors in the calculated dilution ratio. In addition, background aerosols are clearly reached at the dilution ratio of 2371 .

Figure 82 and Figure 83 provide the $\mathrm{CO}_{2}$ map placement and aerosol size distribution results respectively taken on day 10 at an axial placement of 25 foot.

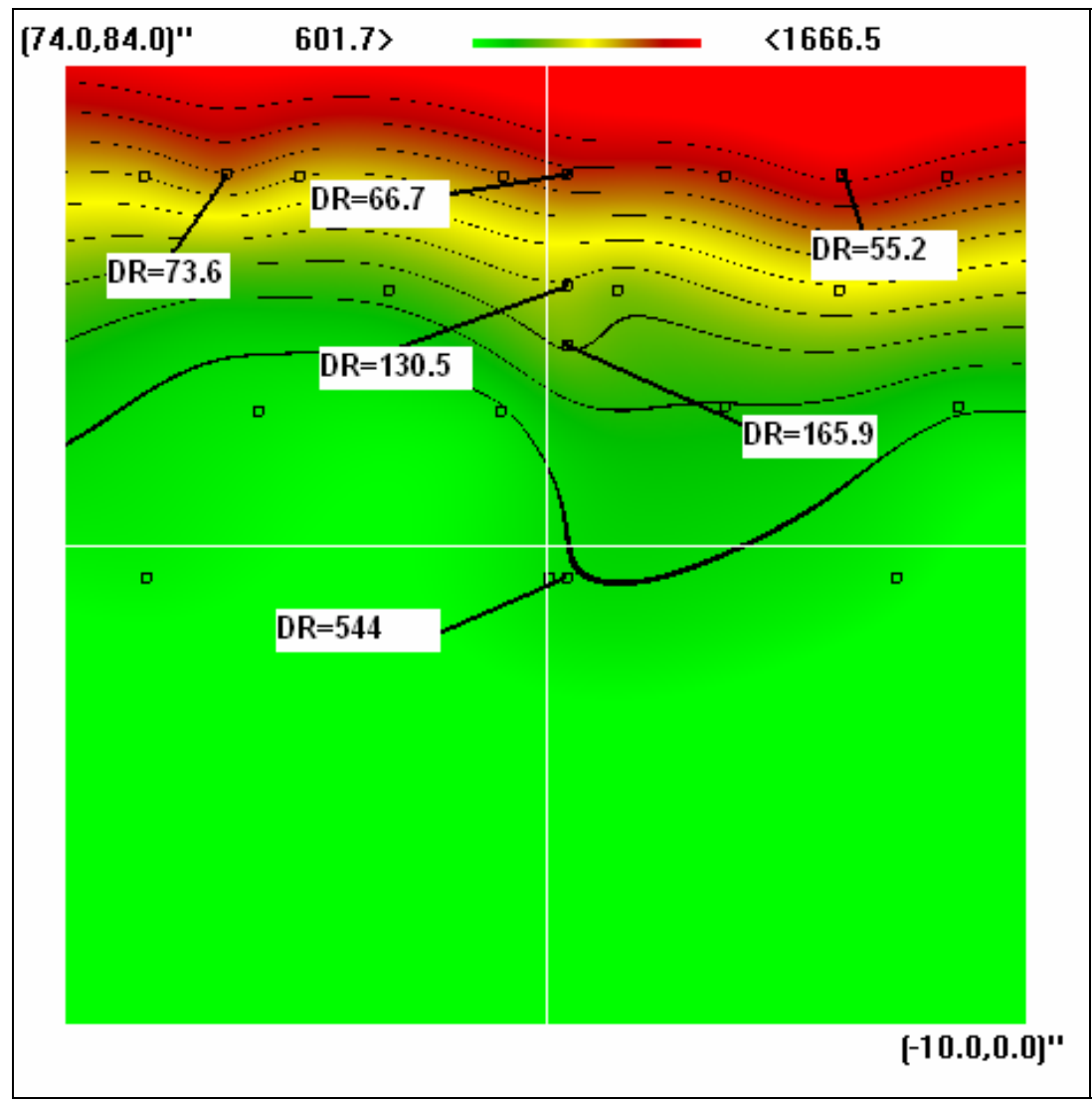

Figure 82 25ft Aerosol Size Distribution Measurement Locations for Figure 83 


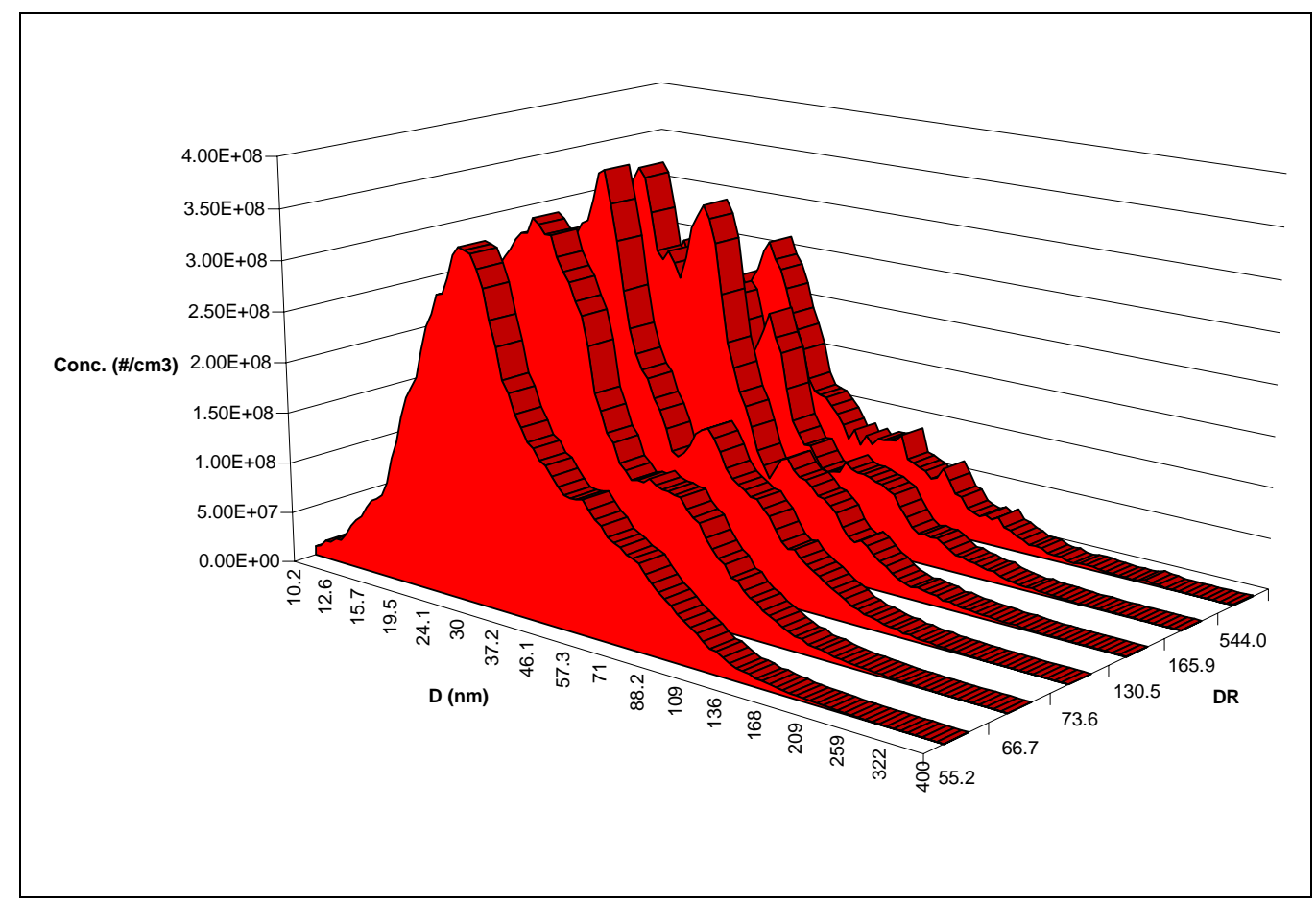

Figure 83 25ft Aerosol Size Distribution Results

As shown in the previous figures, Figure 83 suggests uniform aerosol size distributions throughout the cross-section of the plume. However, these distributions have tended towards smaller particle diameter and larger particle number.

Figure 84 and Figure 85 provide the $\mathrm{CO}_{2}$ map placement and aerosol size distribution results respectively taken on day 10 at an axial placement of 100 foot. 


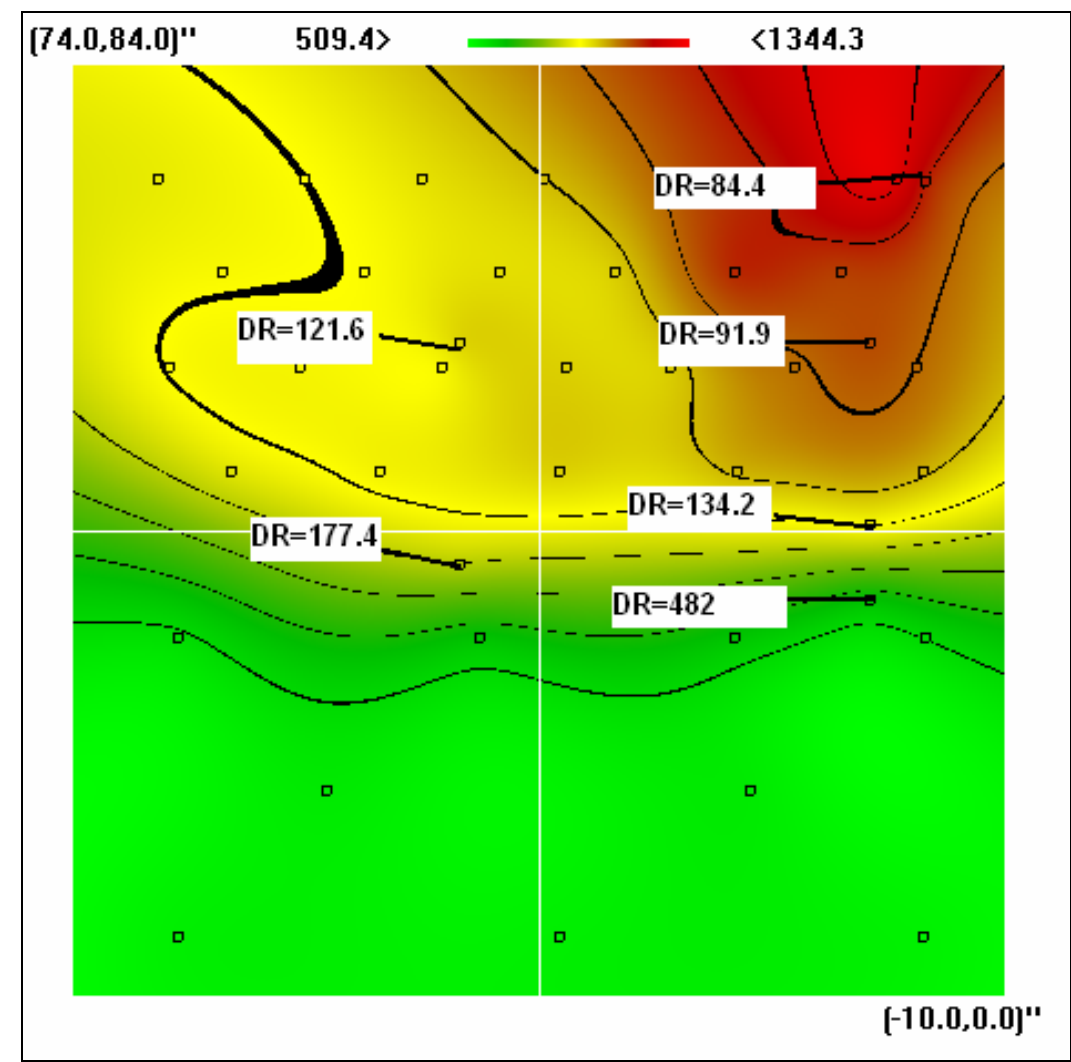

Figure 84 100ft Aerosol Size Distribution Measurement Locations for Figure 85

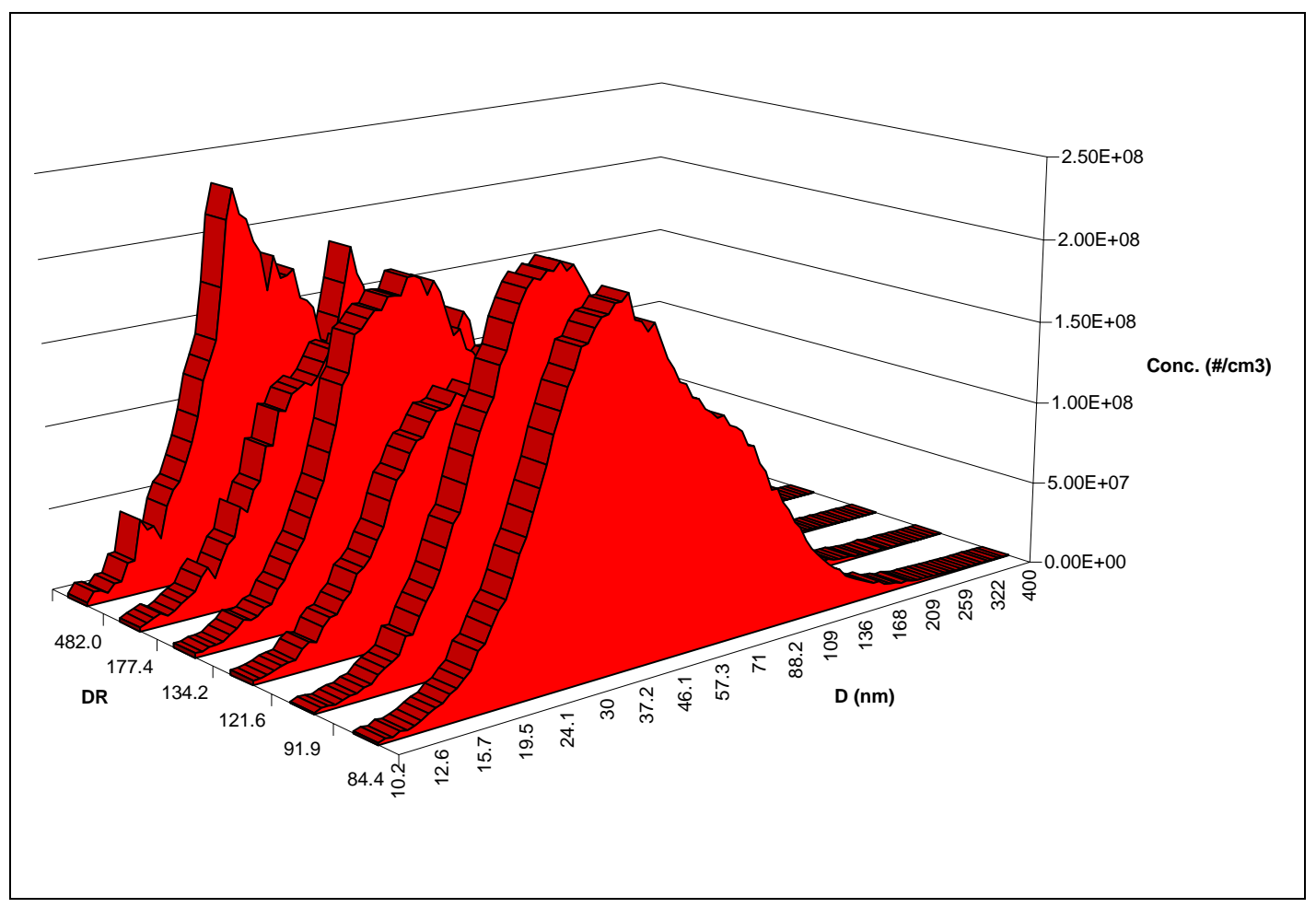

Figure 85 100ft Aerosol Size Distribution Results 
Other than a slight return to larger particle diameters and smaller concentrations, the results of Figure 85 are nearly identical to those of Figure 83. These results suggest a particle evolution with no formation.

Figure 86 and Figure 87 provide the $\mathrm{CO}_{2}$ map placement and aerosol size distribution results respectively taken on day 9 at an axial placement of 125 foot.

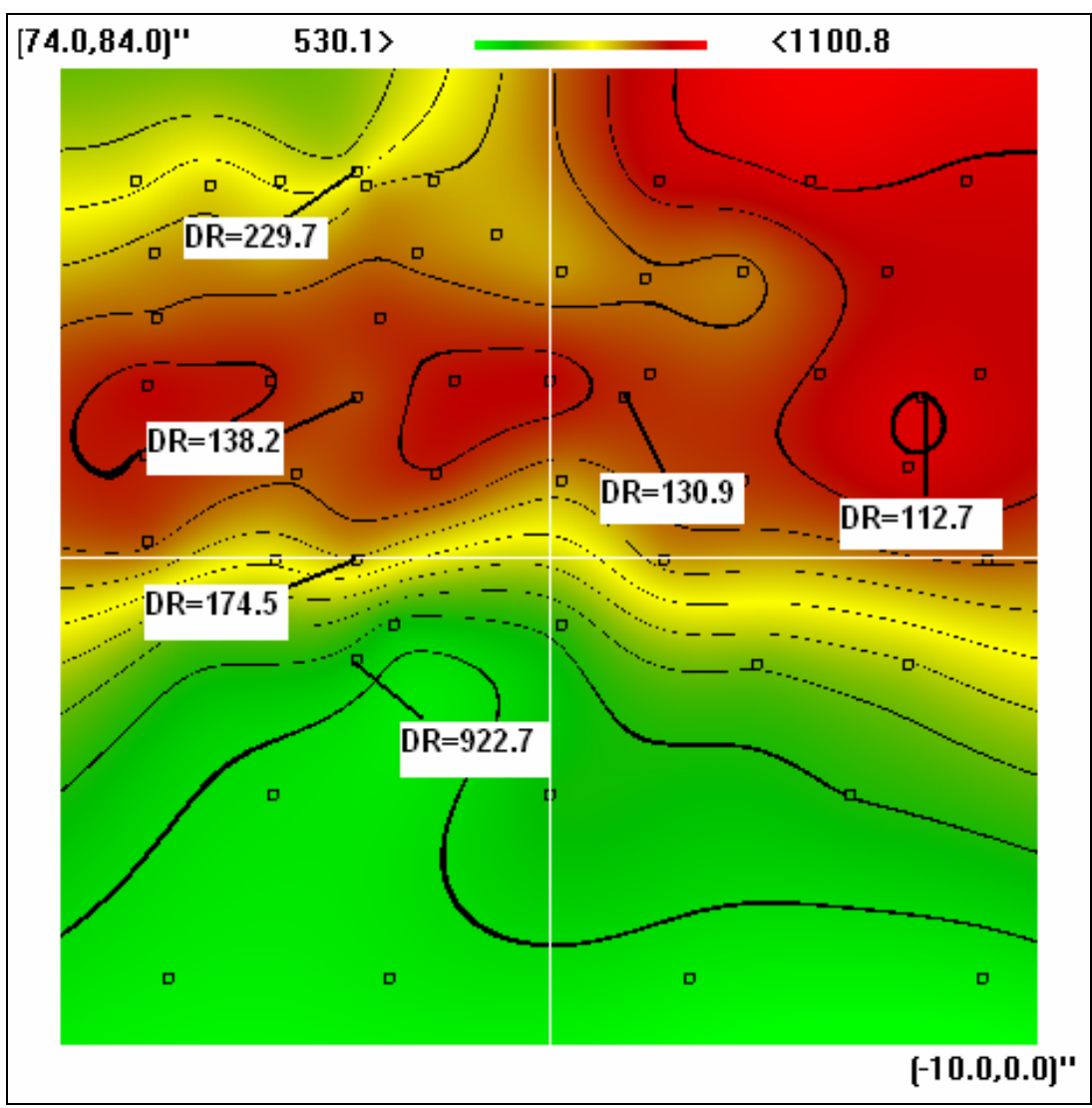

Figure 86 125ft Aerosol Size Distribution Measurement Locations for Figure 87 


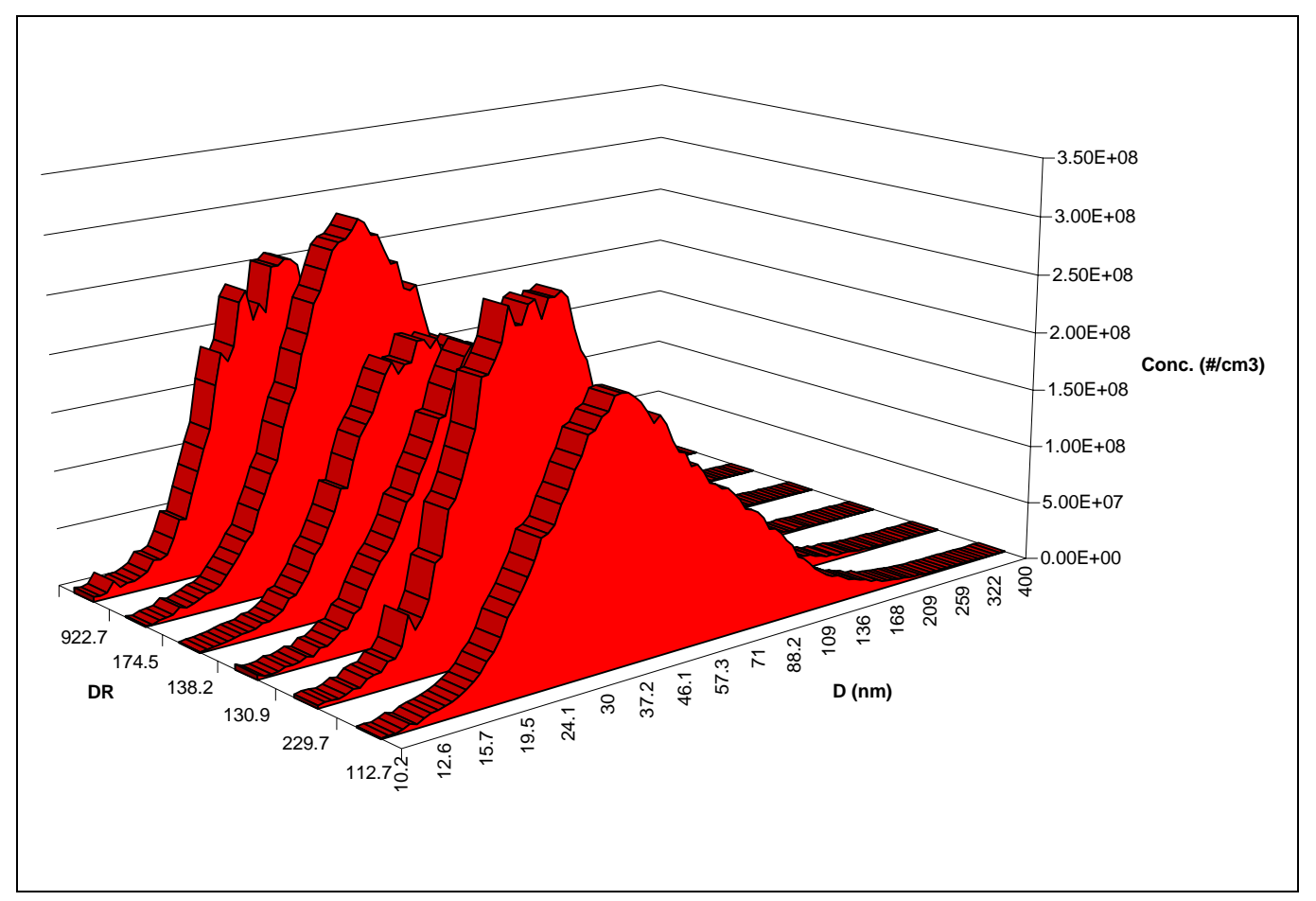

Figure 87 125ft Aerosol Size Distribution Results

As can be seen in Figure 87, the particle distributions of the 125 foot station are in agreement with all other prior stations. As such, it is apparent that no detectible particle formation can be found under I30 operation. This is likely caused by the relatively low exhaust temperatures experienced at this engine setting. As Equation 30 suggests, lower exhaust temperatures, prior to dilution, will significantly reduce the nucleation rate. In addition, a study performed by Lee et al. [46] on a 1.5 liter diesel Isuzu engine showed no discernable nuclei mode at lower engine settings. Consequently, the loss of the nuclei mode in the I30 case may be a particular characteristic of the C240 engine used in this study. In addition, these results are similar to those found in [43], which showed no evolution of the aerosol size distribution under particular engine settings. 


\subsubsection{Effect of Residence (Axial Sample Placement)}

Laboratory residence time studies, such as those performed in [17], typically involve measurements within well established fully mixed flows. In these studies, persistent fluid elements (i.e. those involving a single dilution and temperature value) are tracked to monitor aerosol growth. Due to the persistence of fluid properties, this aerosol growth can be solely attributed to the time the aerosol has been present within the fluid element.

In a complex flow field involving unconstrained mixing such as the one being studied, residence time has a less measurable meaning. In these situations, time can no longer be considered the sole independent variable and must be paired with the path of the fluid element through varying fluid properties. In addition, since paths of fluid elements cross and mixing occurs, residence becomes even more complex as the exact history of a fluid element's aerosol becomes blended with that of other fluid element's aerosols. Even further, non-uniform velocity fields such as those found in Figure 44 make studying residence an even more complex task.

Because of the inability to isolate time within this study, residence will be mentioned in a more qualitative manor. As such, residence will be regarded in terms of the entire plume cross-sections and their axial placement as it relates to plume development time. Figure 88 demonstrates the axial development of aerosols within the plume from stations at 20 to 125 feet while the engine was operated under R75 conditions. This region provides the most informative description of residence within the tunnel, as crosssectional mappings change little with axial placement and as mentioned before crosssectional variations in aerosol size distributions due to dilution are insignificantly irregular beyond 20 feet. 


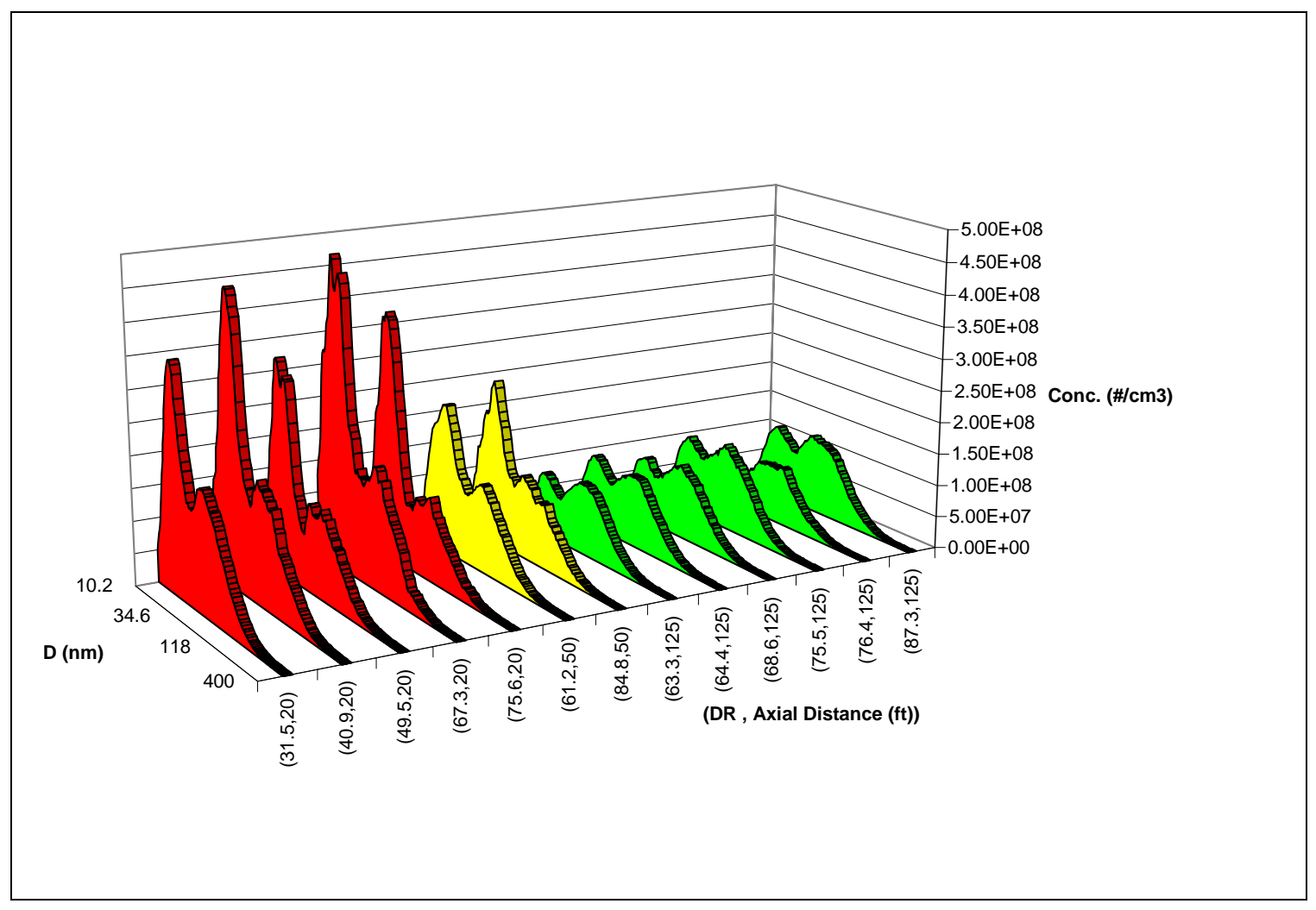

Figure 88 Effect Residence on Aerosol Size Distribution (R75 operation)

As can be seen in Figure 88, aerosol formation (within this axial range) is relatively stagnant with respect to cross-sectional variation in dilution. These approximately uniform cross-sectional aerosol trends demonstrate decay with advancing axial measurement. Peak number concentrations are reduced by a factor of nearly 5 for the 20 to 125 feet range. As can be seen, accumulation mode decay is less observable and remains relatively constant when compared to the decay experienced in the nucleation mode. This same decay is not observed in the initial regions of the tunnel but due to cross-sectional variations in aerosol formation and velocity, can be less easily be attributed to time. Nevertheless, a single dilution ratio of 35 was paired with accompanying aerosol size distributions (interpolated as needed) for the first 20 feet of 
the tunnel. These distributions were taken from data acquired under R75 conditions and are given in Figure 89.

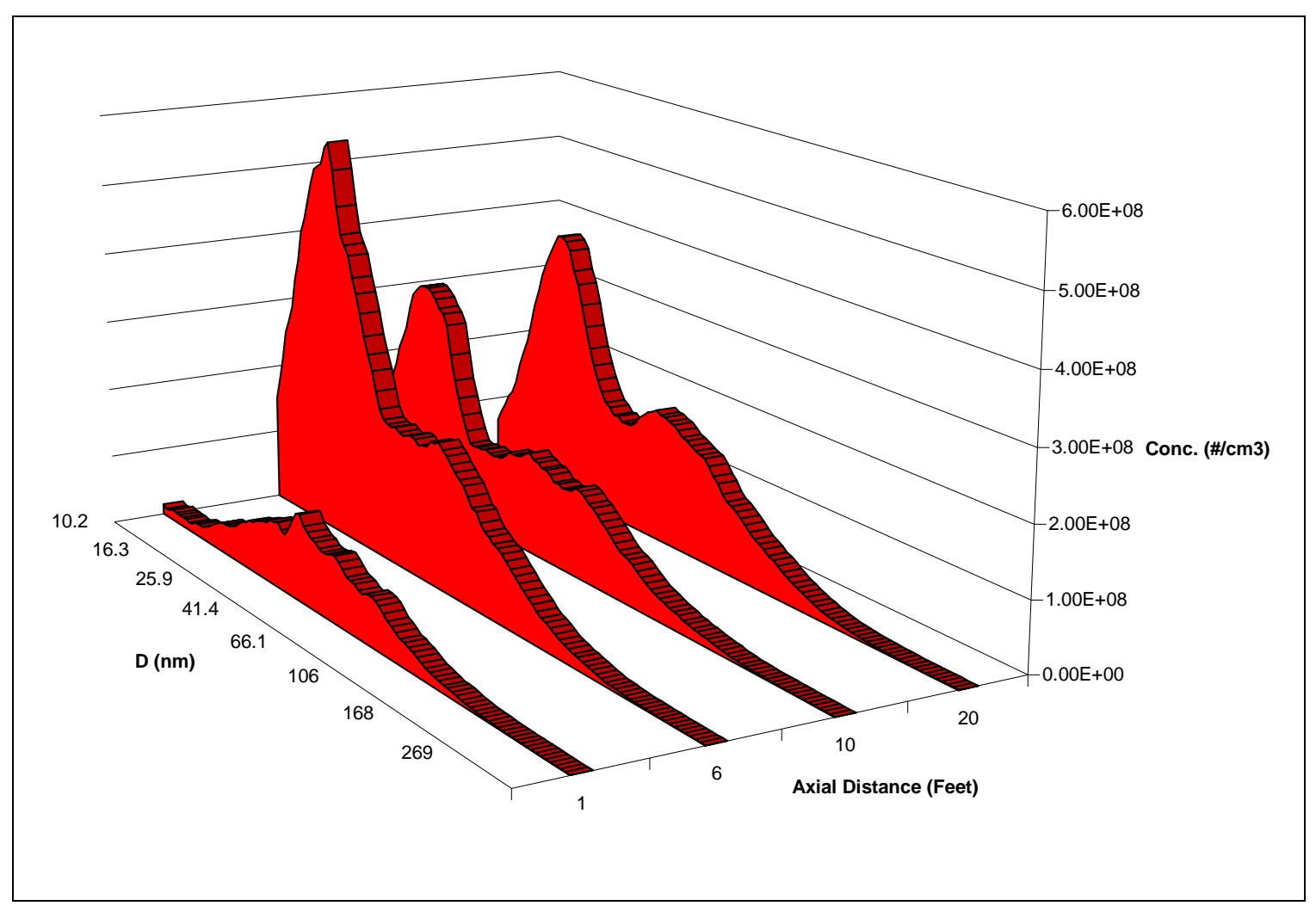

Figure 89 Effect of Residence on Aerosol Formation (DR=35)

As can be seen in Figure 89, there is a sharp increase in the nuclei mode particles up to an axial distance of $\sim 6$ feet. This peak is met by a less drastic decrease at $\sim 10$ feet which remains relatively unchanged up to 20 feet. From 20 feet on, all distributions regardless of dilution ratio are shown to experience losses in the nuclei mode. Unfortunately, these results do not originate from a single exhaust path, which might further alter their connection to time. 


\subsection{Uncertainty}

\subsubsection{Scalar Mapping}

The following section offers a description of the uncertainty that can be expected in the scalar mapping results of this study. Of particular interest are the $\mathrm{CO}_{2}$ maps, which were used exclusively to order and scale aerosol size distribution data. As this is an averaging study, errors in scalar mapping results can originate from inadequate averaging times, low spatial resolution, and / or biasing errors within the instrumentation and environment of the tunnel.

Averaging time was used to remove measurement fluctuations caused by natural plume oscillation and random errors within the instrumentation. These times were not intended to remove events that occur over periods spanning more than a few seconds, such as the introduction of foreign objects within the tunnel, gradual exhaust misalignment, and major changes in tunnel flow. These events can typically be drawn out through averaging, but require large sampling times and can significantly reduce map similarity to instantaneous measurements. Some events however, proceed in a biased manner which cannot be drawn out through averaging at all.

The effect of uncontrolled long duration events has been demonstrated in a number of the scalar mapping results of Section 3.2. Figure 90 describes a controlled study which was used to determine the influence of placing foreign objects in the vicinity of the plume. 

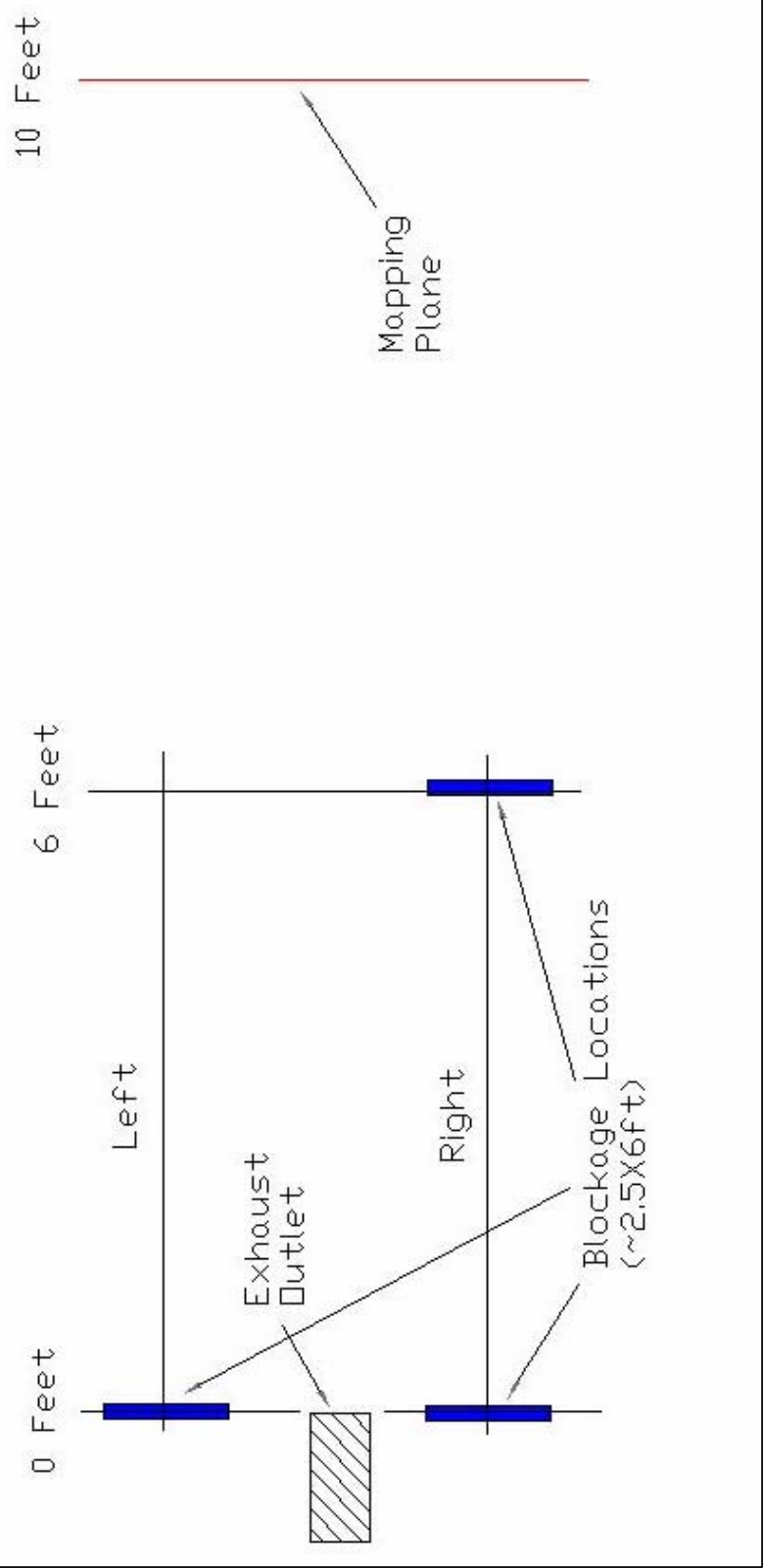

Figure 90 Schematic of Study to Determine Foreign Object Interference on Mapping Results.

In this study, a blockage approximately 2.5 feet wide by 6 feet tall was placed at a number of locations to the right, left and downstream of the exhaust outlet. Scalar exhaust maps were taken downstream for each of the blockage arrangements. The $\mathrm{CO}_{2}$ mapping results of this study are shown in Figure 91. 


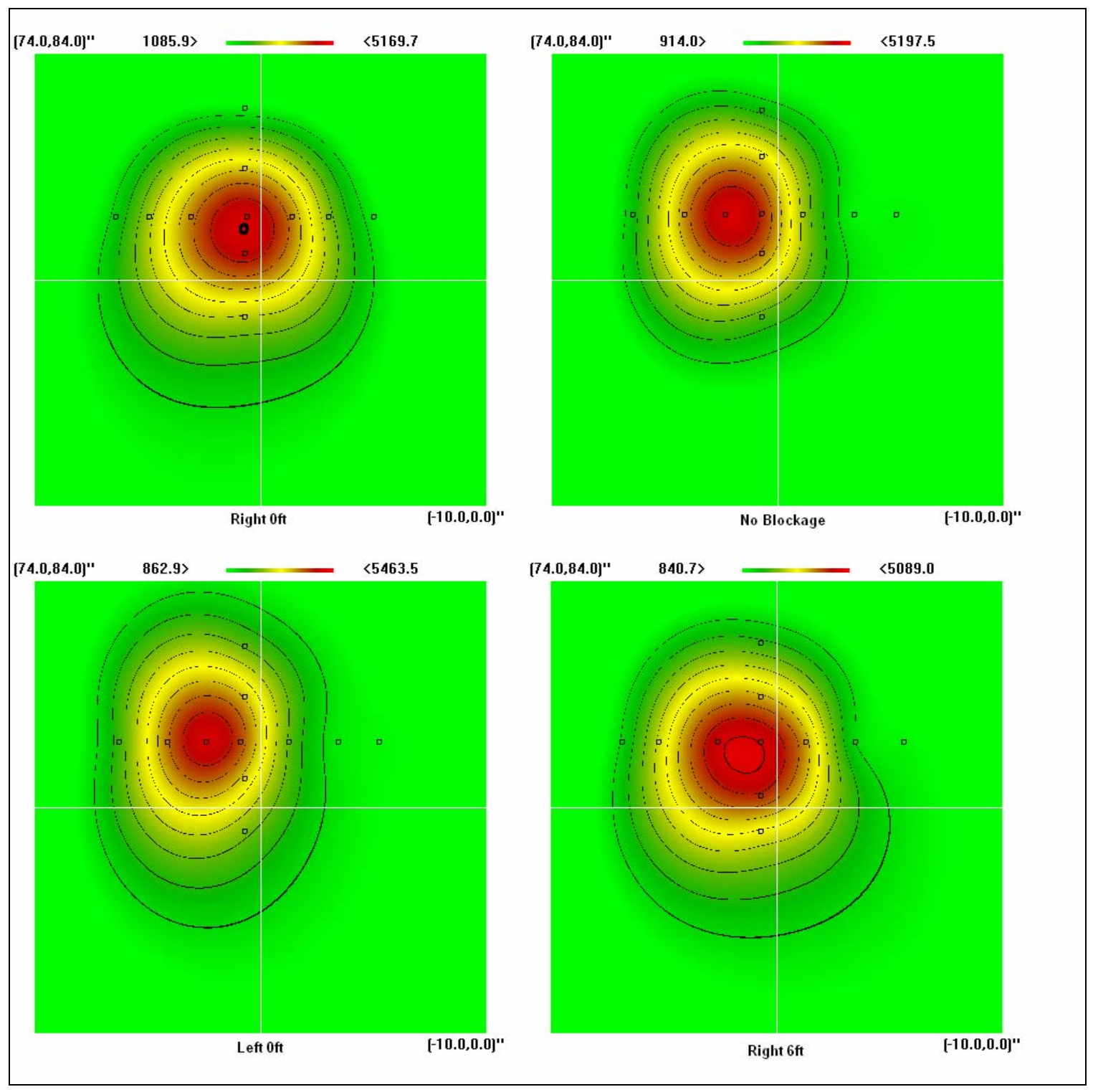

Figure $91 \mathrm{CO}_{2}$ Mapping Results for Various Blockage Arrangements.

As can be seen in Figure 91, there is a definite response in the location of the plume to the introduction of blockages within the tunnel. Blockages within the left side of the tunnel have less influence on plume position, as they are overshadowed by the present of the dynamometer and engine. Blockages to the right side of the tunnel are much more apparent. These are made even more significant with distance from the measurement plane, as this allows more bending of the flow to occur in response to the blockage. 
The travel experienced in all four arrangements of Figure 91 spans $\sim 7$ inches. This travel is enough to span over $30 \%$ of the map scale for this particular case. As such, scans in which irregular blockage issues cannot be resolved may result in irregular waviness and lack of predictive value (uncertainty) in the scalar exhaust maps.

Scalar exhaust maps may also lack predictive value due to low spatial resolution. Adequate spatial resolution can be seen in terms of map convergence. Assuming an average scalar field exists, it should be converged to upon ample sample time, number and placement. Showing the convergence of a 3 dimensional map can be cumbersome and confusing. However, showing the convergence along an arbitrary curve drawn through the scalar field should suffice in demonstrating the convergence of the entire map. Figure 92 demonstrates this technique applied to the data taken on day 7 at an axial placement of 10 feet.

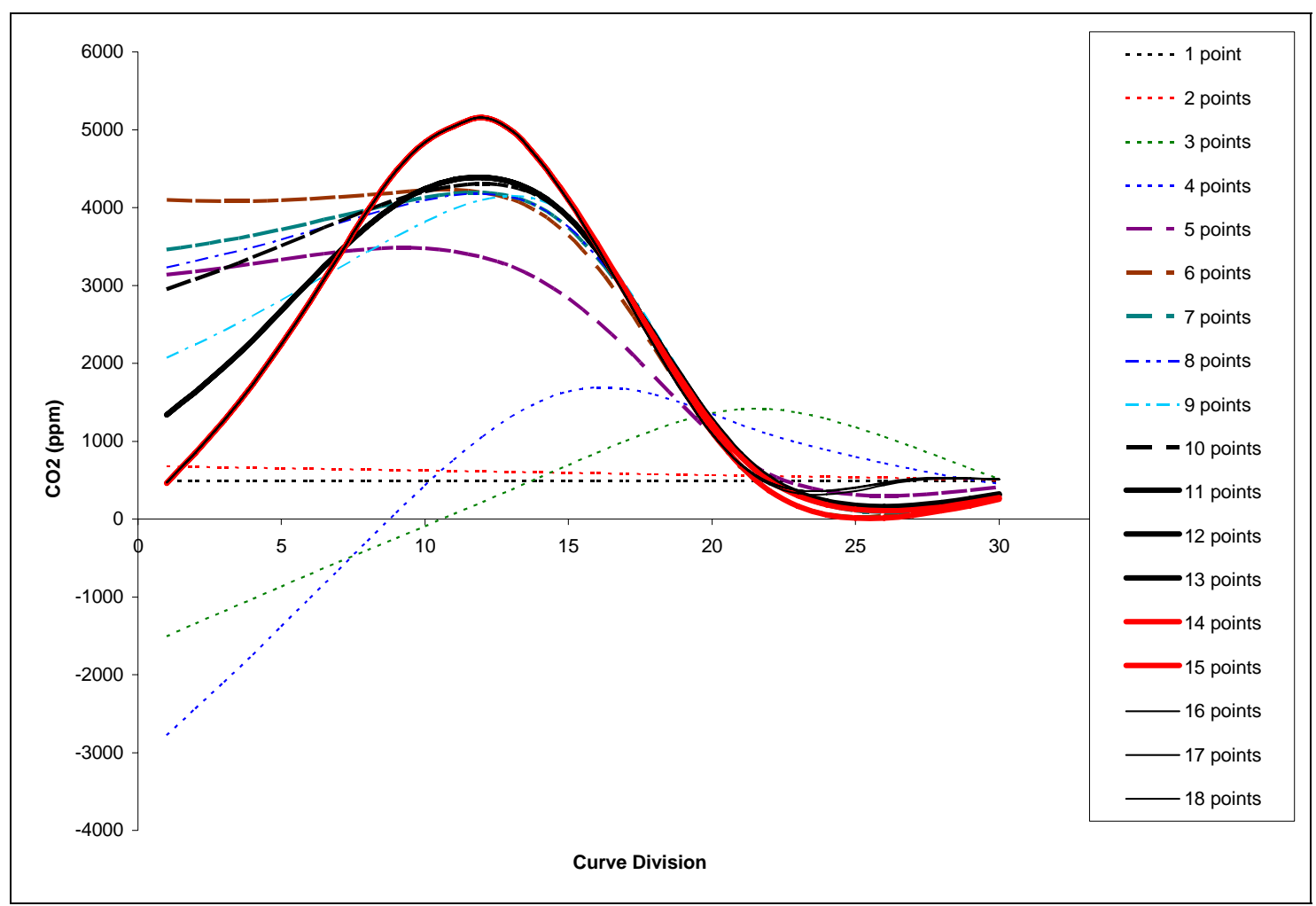

Figure 92 Convergence of $\mathrm{CO}_{2}$ Map Along an Arbitrary Line. (Taken on day 7 at 10 feet) 
As can be seen, Figure 92 shows the resulting $\mathrm{CO}_{2}$ distributions along a common arbitrary curve through the mapping plane for the addition of each sample point collected. This figure suggests a strong convergence of the map. In fact, the distributions for the addition of the last 7 sample points are nearly identical.

Figure 93 demonstrates the convergence of the data taken on day 6 at an axial placement of 20 feet.

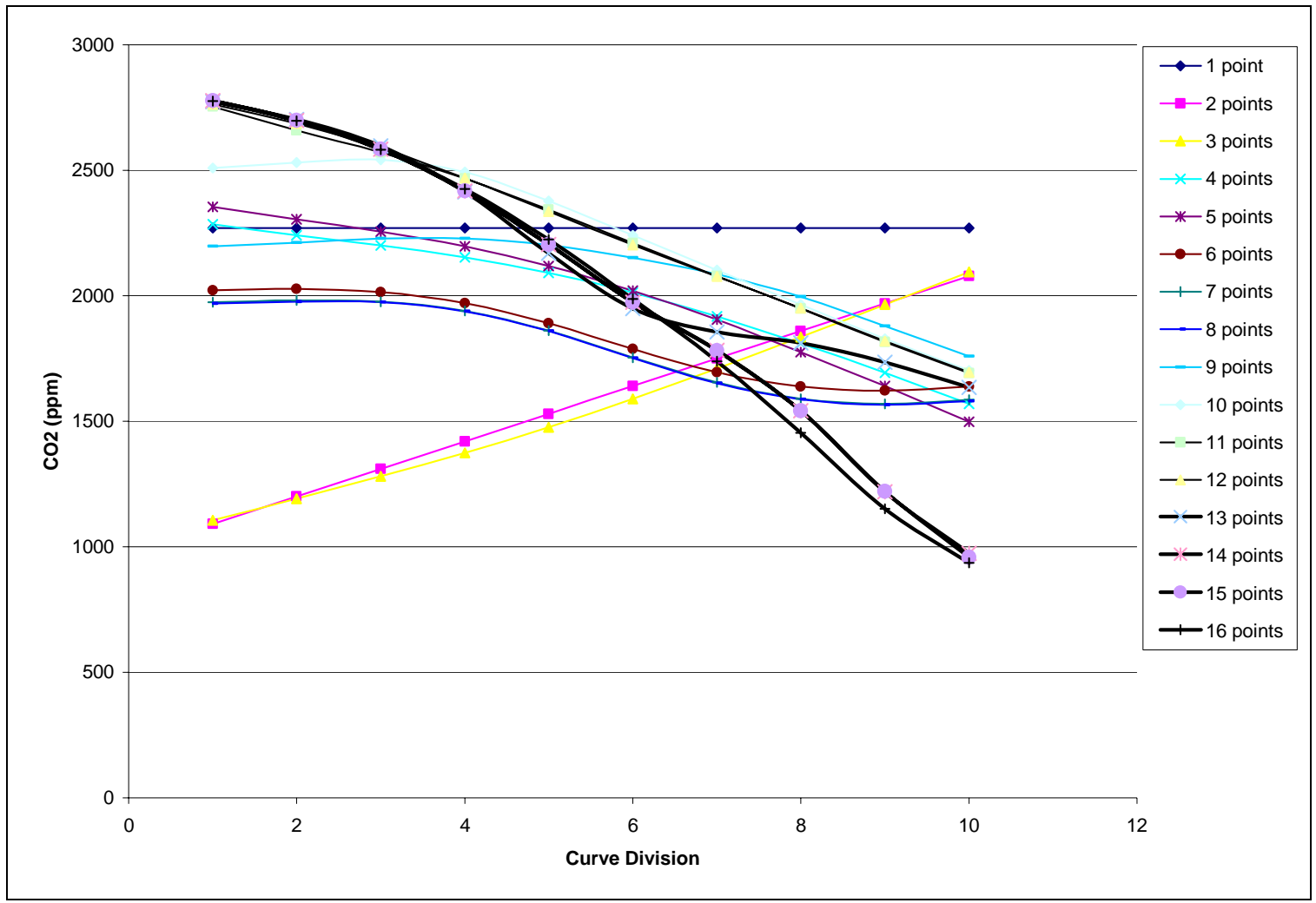

Figure 93 Convergence of $\mathrm{CO}_{2}$ Map Along an Arbitrary Line. (Taken on day 6 at 20 feet)

As in Figure 92, Figure 93 suggest strong convergence of the $\mathrm{CO}_{2}$ mapping results. The addition of the last 3 sample points has no perceivable affect on the cumulative distribution.

Instrument bias and random errors are another source of map uncertainty. Random errors are of no concern in this study since they are neutralized during averaging. 
However, bias errors can result in map scale error and geometrically distorted contours. If bias errors occur uniformly across the measurement span, no distortions will be found in the geometry of the plume. In addition, calculated dilution ratios will be fully accurate. Non-uniform bias errors however, will result in distorted maps and erroneous dilution ratios. These errors will also be magnified by the nonlinear nature of dilution ratio. This observation is likely one of the major issues causing outer plume trend irregularity in Section 3.3. One possible cause of biasing error could originate from inadvertent slippage of the incoder gearing system due large vibrations in moving the test apparatus between stations. This issue can be resolved in future studies through the use of more robust set screws and shock absorers within the encoder components.

\subsubsection{Aerosol Size Distribution}

The following section describes the uncertainty to be expected in the aerosol size distribution results of this study. The reader is referred to the SMPS user's manual for the specific uncertainty found in the instrumentation. This section provides raw (unscaled) SMPS measurement results and discuss the validity of averaging. Figure 94 shows the average aerosol size distributions and error bars taken at 6 feet on day 5 . 


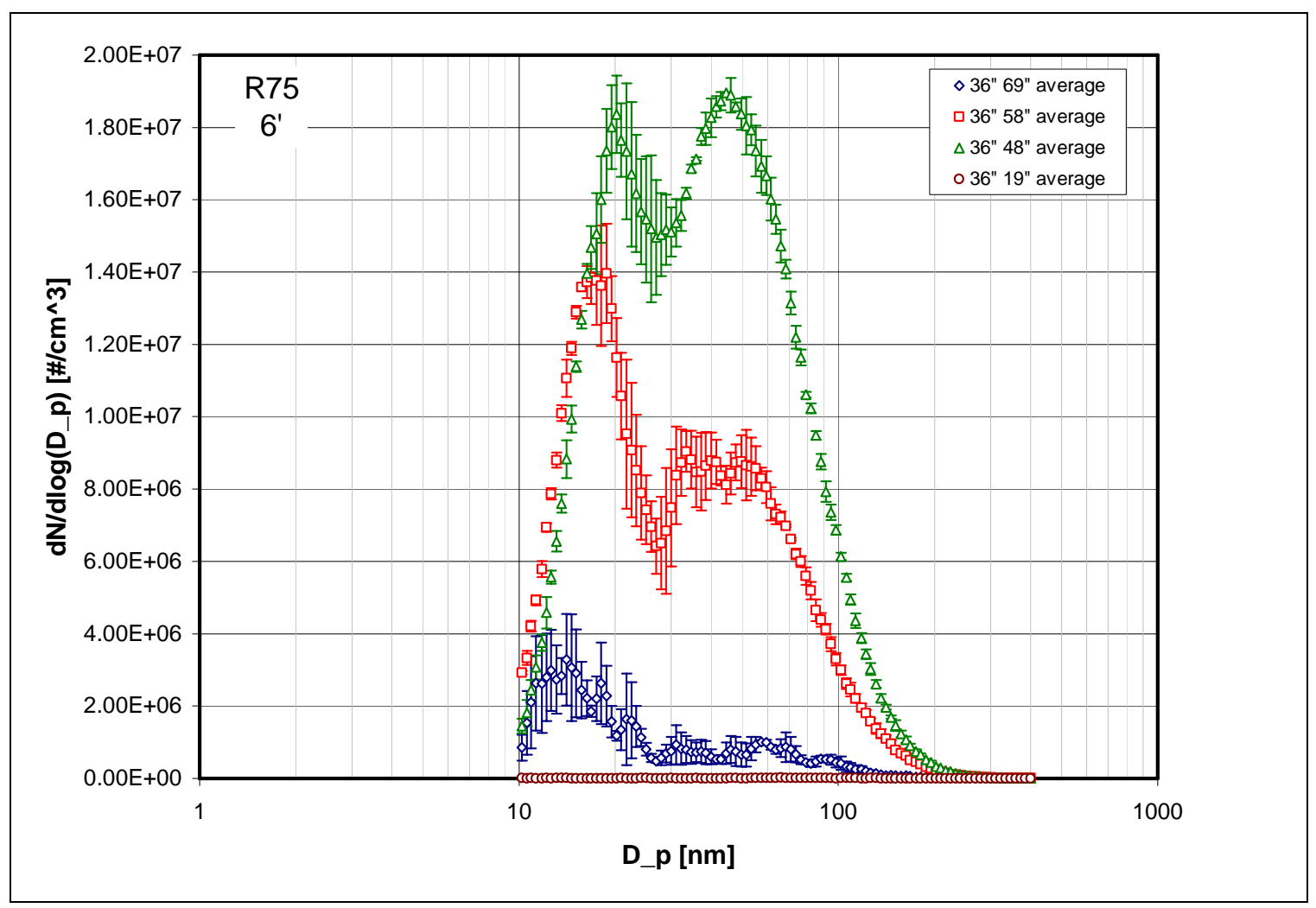

Figure 94 Aerosol Size Distribution Results with Error Bars (day 5 6ft)

As can be seen in Figure 94, the instantaneous aerosol size distributions at 6 feet under R75 operation can be expected to be relatively close to the acquired averages.

Figure 95 shows the average aerosol size distributions and error bars taken at 10 feet on day 5 . 


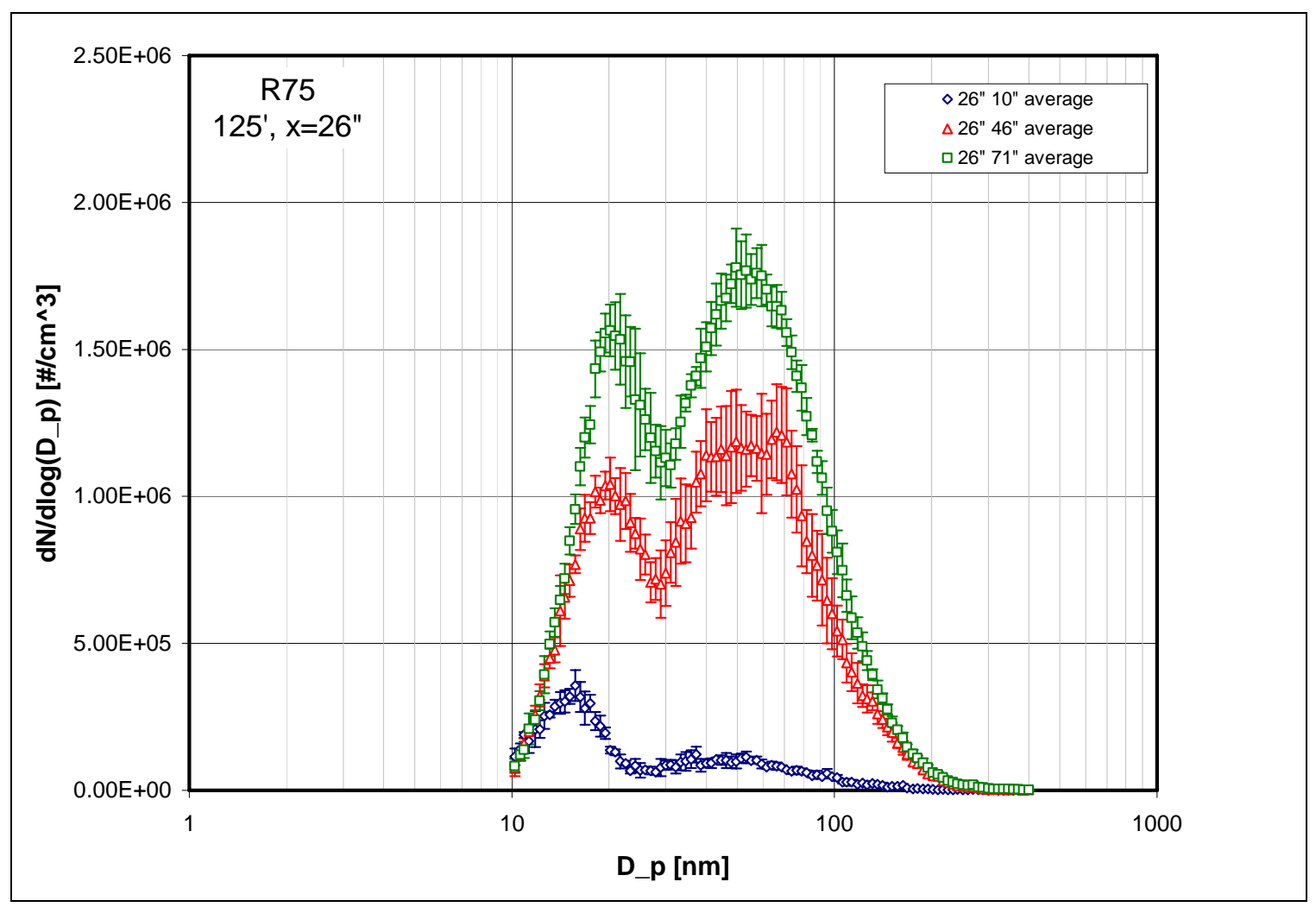

Figure 95 Aerosol Size Distribution Results with Error Bars (day 5 125ft)

With the exception of the second data set, the distributions of Figure 95 appear very

stabile. The second data set has most of its variation present in the accumulation mode.

This set was taken towards the lower border of the stratified layers and may suggest flow instability.

Figure 96 shows the average aerosol size distributions and error bars taken at 25 feet on day 10 . 


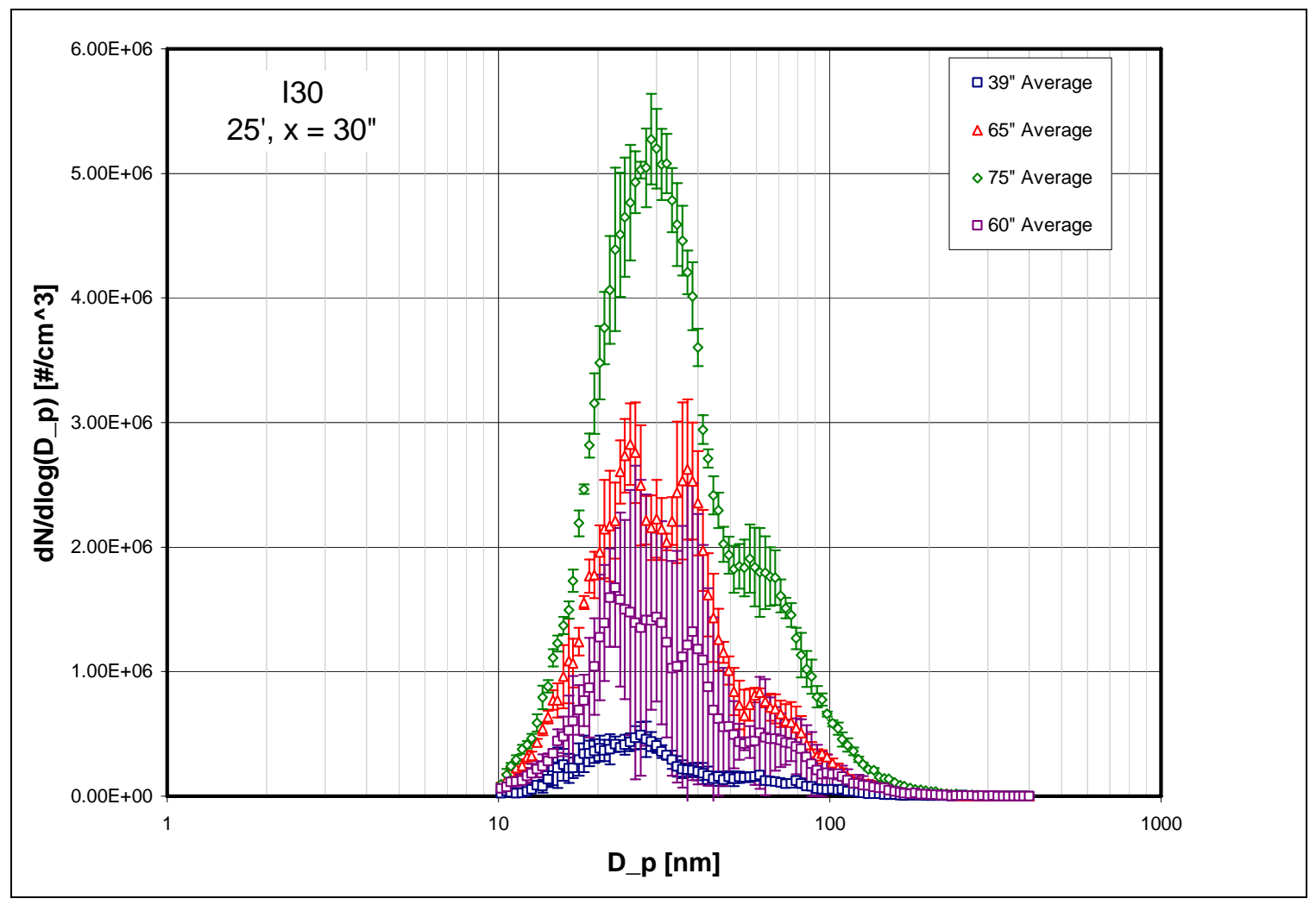

Figure 96 Aerosol Size Distribution Results with Error Bars (day 10 25ft)

The distributions of Figure 96 appear highly irregular. This is particularly true for the third distribution. In fact, measurements other than temperature all appeared unstable for I30 operation. Smoothing strategies other than averaging were not incorporated to allow an indication of instabilities within the plume. 


\section{Conclusions}

Results of this study are similar to those of other aerosol studies and indicate a strong connection of the nuclei mode to dilution ratio and residence time. However, the use of a ventilated mine tunnel with a dynamometer controlled diesel engine has not been found in any published emissions work. In addition, no other study has been found to use precision mapping techniques for the strategic positioning of aerosol samples. This approach has been demonstrated to provide a highly efficient means of gathering precise and comprehensive diesel exhaust aerosol evolutionary trends. Furthermore, the "realworld” flow conditions, and PM formation and evolutionary trends found in this study would be difficult to achieve in an engine laboratory setting using conventional techniques. This difficulty is mostly due to the fact that the approach used in this study had the ability of maintaining the natural development of exhaust variables within the

plume. Furthermore, the precise spatial detail of the exhaust plume suggests that aerosol formation cannot be resolved without the use of accurate mapping techniques. Studies which neglect this observation will incur a great deal of uncertainty in intended sample placement with even further uncertainty in observed aerosol evolutionary trends. Under R75 operation, aerosol formation occured within the first 20 feet of plume development. However, these formation characteristics were not found uniform across the plume. Particular dilution ratios (i.e. $\mathrm{DR}=35$ ) experienced peak growth as early as 6 feet from the exhaust source while others continued to much greater depths. With added axial resolution, it is expected that these numbers may be found even more diverse. Beyond 20 feet however, the nuclei mode began to experience appreciable uniform losses throughout the plume. By 125 feet, the nuclei mode was reduced, in all regions of 
the plume, by nearly one order of magnitude from its peak recorded levels. In spite of changes found in the nuclei mode, the accumulation mode remained relatively constant throughout the entire 200 foot test region.

Under intermediate $30 \%$ operation, no nuclei mode was found anywhere in the entire tunnel test domain. This may be a particular characteristic of the Isuzu C240 engine used during this study. In addition, the nuclei mode may have been lost due to the low exhaust temperatures experienced at this engine setting. These may have been low enough to extinguish any large saturation ratio tendicies towards nucleation. The nuclei mode may have originated if the exhaust system was made shorter, reducing heat transfer prior to dilution. 


\section{Future Recommendations}

It is recommended that future aerosol studies, of naturally occurring exhaust plumes, be conducted through the use of precise spatial mapping devices. This technique can increase the efficiency, quality and quantity of aerosol evolutionary trends found in emissions work. In addition, precise spatial mapping may assist in determining empirical models for the spatial and physical connections present within the exhaust plume.

Future studies may also be conducted to explore instantaneous multi-dimensional maps of exhaust plumes, which might be accomplished through the use of high speed electronic valves. These maps can be used to reduce physical and spatial dependencies, within the plume, even further. In addition, instantaneous mapping will alleviate some of the need to maintain environmental averages.

The results of this study suggest that aerosol formation occured within the first 20 feet of plume development. This region (0 to 20 feet) will require further resolution if exact formation characteristics are to be identified. However, it should be understood that physical and spatial occurrences are not decoupled through mapping. As such, without significant resolution, it cannot be known whether gradients found downstream of the exhaust source are the result of local physical process or the spatial continuation of events that occurred earlier. Results of the fine resolution aerosol study suggest that aerosol formation has peaked somewhere around 6 feet, but are not significant enough to form any definite conclusions.

It is recommended that future emissions studies explore exhaust plume mapping under more controlled (i.e. control over ambient ventilation variables) environments and to an even higher degree of resolution and accuracy. These studies may be performed within 
the dimensions observed in the tunnel for the first 20 feet of development. Ideally, these dimensions should be oriented in an inclined direction to avoid wall interactions from buoyantly driven exhaust. Under controlled laboratory environments, the characteristics of exhaust plume development can be understood with even more certainty than found in this study. 


\section{References}

1. Kittelson D. B., and Abdul-Khalek I., (1999), "Formation of Nanoparticles During Exhaust Dilution,” EFI Members Conference: Fuels, Lubricants, Engines, \& Emissions. 18-20, January

2. Schnakenberg Jr. G., and Bugarski A., (2002), "Review of Technology Available to the Underground Mining Industry for Control of Diesel Emissions," IC 9462: 52.

3. Kittelson D. B., Johnson J., Watts W., Wei Q., Drayton M., Paulsen D., and Bukowiecki N., "Diesel Aerosol Sampling in the Atmosphere," SAE paper 2000-012212, 2000.

4. Abdul-Khalek I., and Kittelson D.B., (1995), "Real Time Measurement of Volatile and Solid Exhaust Particles Using a Catalytic Stripper,” SAE paper No. 950236.

5. Brown J. E., Clayton M. J., Harris D.B., and King Jr. F., (2000), "Comparison of the Particle Size Distribution of Heavy-Duty Diesel Exhaust Using a Dilution Tailpipe Sampler and an In-Plume Sampler During On-Road Operation,” Journal of Air \& Waste Management Association, Vol. 50, pp. 1407-1415.

6. Bagley, S., Baumgard, K., Gratz, L., Johnson, J., and Leddy, D. (2004), “Effects of Fuel Modification and Emission Control Devices on Heavy-Duty Diesel Engine Emissions”, Reaserch report number 76, The Health Effects Institute, Houghton, MI.

7. Seinfeld, J. (1998), ATMOSPHERIC CHEMISTRY AND PHYSICS, John Wiley \& Sons. Inc., New York.

8. von Smoluchowski, M. (1916), "Versuch Einer Mathematischen Theorie Der Koagulationskentik Kolloidaler Losungen”, Zeitschrift fur physik, Chemie 92: 129-168.

9. Saffman, G., and Turner, S. (1956), "On the Collision of Drops in Turbulent Clouds", Journal of Fluid Mechanics, 1, pp. 16-30.

10. Wunsche, B., and Tempero, E. (2004), “A Comparison and Evaluation of Interpolation Methods for Visualizing Discrete 2D Survey Data”, Australian Computer Society, Inc. Conferences in Research and Practice in Information Technology, Vol. 35. 11. Dyn, N. (1989), "Interpolation and Approximation by Radial and Related Functions”, in C.K. Chui, L.L. Schumaker and J.D. Ward, eds, APPROXIMATION THEORY VI, Vol.1, Academic Press, pp. 211-234. 
12. Hoscheck J., and Lasser D. (1992), FUNDAMENTALS OF COMPUTER AIDED

GEOMETRIC DESIGN, $2^{\text {nd }}$ edition, AK Peters Ltd., Wellesley, MA 02181, chapter 14, pp. 572-601.

13. Isuzu Diesel, C240 > MAIN SPECIFICATIONS, Internet Reference, www.continentalengines.com/pdf_files/isuzu_c240_ind.pdf

14. Canagaratna M. R., Jayne J., Ghertner D., and Herndon S. (2004), "Chase Studies of Particulate Emissions from In-Use New York City Vehicles,” Aerosol Science

Technology, 38, 555-573, doi: 1080/02786820490465504.

15. Allen M. D., and Raabe O. G. (1982), "Re-Evaluation of Millikan’s Oil Drop Data for the Motion of Small Particles in Air,” Journal of Aerosol Science, 13(6): 537-547. 16. Brock R., "On the Theory of Thermal Forces Acting on Aerosol Particles,” Journal of Colloid Science, Vol. 17, pp. 768-780, 1962.

17. TSI, Inc., MODEL 3936 SMPS INSTRUCTION MANUAL, P/N 1922796, September 2003.

18. TSI, Inc., MODEL 3007 CONDENSATION PARTICLE COUNTER OPERATION AND SERVICE MANUAL, P/N 1930035, August 2002.

19. Mathieu J., and Scott J. (2000), AN INTRODUCTION TO TURBULENT FLOW, Cambridge University Press, chapter 1, pp. 22.

20. Oosthuizen P., and Naylor D. (1999), INTRODUCTION TO CONVECTIVE HEAT TRANSFER ANALYSIS, McGraw-Hill Companies, Inc., pp. 606.

21. Tribsch G. F., and Sapko M. J. (1990), "Lake Lynn Laboratory: A State-of-the-Art Mining Research Laboratory," Procedings of International Symposium on Unique Underground Structures, Denver Colorado, June 12-15.

22. Von Mises R. (1959), THEORY OF FLIGHT, Dover Publications, Inc., New York, New York, pp. 78.

23. Vennard J. (1952), ELEMENTARY FLUID MECHANICS, pp. 328.

24. Kays W., and Crawford M. (1993), CONVECTIVE HEAT AND MASS

TRANSFER, John Wiley and Sons, Inc., New York, New York, pp. 334.

25. Mills A. (1999), BASIC HEAT AND MASS TRANSFER, Prentice Hall, New Jersey, Upper Saddle River, pp. 269. 
26. Liepmann H., and Roshko A. (1957), ELEMENTS OF GASDYNAMICS, John Wiley and Sons, Inc., New York, New York, pp. 348.

27. Rader D. J., and Marple V. A. (1988), "A Study of the Effects of Anisokinetic Sampling," Aerosol Science Technology, 8, 283-299.

28. Vincent J. H., Emmett P. C., and Mark D. (1985), “The Effects of Turbulence on the Entry of Airborne Particles Into a Blunt Dust Sampler,” Aerosol Science Technology, 4, 17-29.

29. Wiener R. K., Okazaki K., and Willeke K. (1988), “Influence of Turbulence on Aerosol Sampling Efficiency,” Atmospheric Environment, 22, 917-928.

30. Ayala A., Olson B., and Cantrell B. (2002), "Working Quality Assurance Project Plan for Diesel Aerosol Sampling Methodology (CRC Project E-43),” Available from the Coordinating Research Council, Alpharetta, Georgia.

31. Willeke K., and Baron P. (1993), AEROSOL MEASUREMENT PRINCIPLES TECHNIQUES AND APPLICATIONS, Van Nostrand Reinhold, New York.

32. Hangal S., and Willeke K. (1990), "Overall Efficiency of Tubular Inlets Sampling at 0-90 Degrees from Horizontal Aerosol Flows,” Atmospheric Environment, 24, 23792386.

33. Belyaev S. P., and Levin M. L. (1972), "Investigation of Aerosol Aspiration by Photographing Particle Tracks Under Flash Illumination,” Journal of Aerosol Science, 3: 127-140.

34. Kommer E. (2003), "Variations of Engine Particulate Matter in a Miniature Dilution Tunnel,” Masters Thesis, University of Maryland.

35. Fuchs N. A. (1964), "The Mechanics of Aerosols,” Oxford, Pergamon.

36. Holman J. P. (1972), HEAT TRANSFER, McGraw-Hill, New York, New York.

37. Liu B., and Agarwal K. (1974), "Experimental Observation of Aerosol Deposition in Turbulent Flow,” Journal of Aerosol Science, 5: 145-155.

38. Crane R. I., and Evans R. L. (1974), "Inertial Deposition of Particles in a Bent Pipe," Journal of Aerosol Science, 8: 161-170.

39. Ye Y., and Pui D. (1990), "Particle Deposition in a Tube with an Abrupt Contraction,” Journal of Aerosol Science, 21: 29-40. 
40. McMurry P., and Rader D. (1985), “Aerosol Wall Losses in Electrically Charged Chambers,” Journal of Aerosol Science, 4: 249-268.

41. Friedlander S. (1977), SMOKE, DUST, AND HAZE, John Wiley and Sons, Inc., New York, New York.

42. Waldmann L., and Schmitt K. (1966), THERMOPHORESIS AND

DIFFUSIOPHORESIS OF AEROSOLS, Academic Press, New York, New York, 137162.

43. Gautam M., Clark N., Metha S., Boyce J., Rogers F., and Gertler A. (2004), "Concentrations and Size Distributions of Particulate Matter Emissions from a Class-8 Heavy-duty Diesel Truck Tested in a Wind Tunnel,” $\underline{\text { SAE Transactions, Journal of Fuels }}$ and Lubricants, 1615-1634.

44. Norment H. (1988), “Three-dimensional Trajectory Analysis of Two Drop Sizing Instruments: PMS OAP and PMS FSSP,” Journal of Oceanic Technology, 5, 743-756. 45. Hinds W. (1982), AEROSOL TECHNOLOGY, John Wiley and Sons, Inc., New York, New York.

46. Lee D., Miller A., Park K., and Zachariah M. (2006), "Effects of Trace Metals on Particulate Matter Formation in a Diesel Engine: Metal Contents from Ferrocene and Lube Oil,” International Journal of Automotive Technology, 7: 667-673. 


\section{Appendix A (R75 Temperature Maps)}

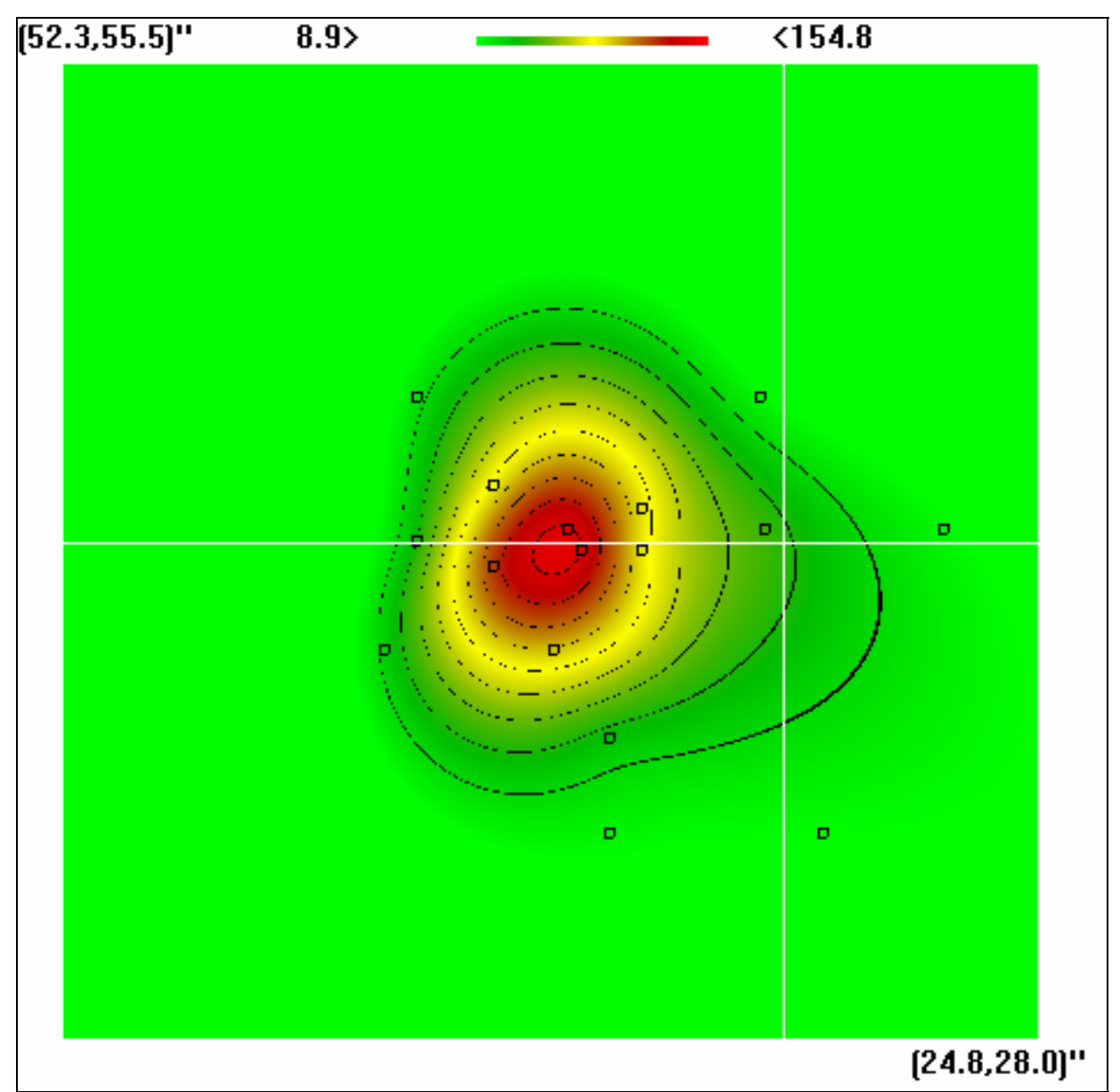

Figure 97 Temperature Mapping Results for an Axial Placement of 11 inches (Taken on Day 7).

(Color Scale Refers to Temp. (C)) 


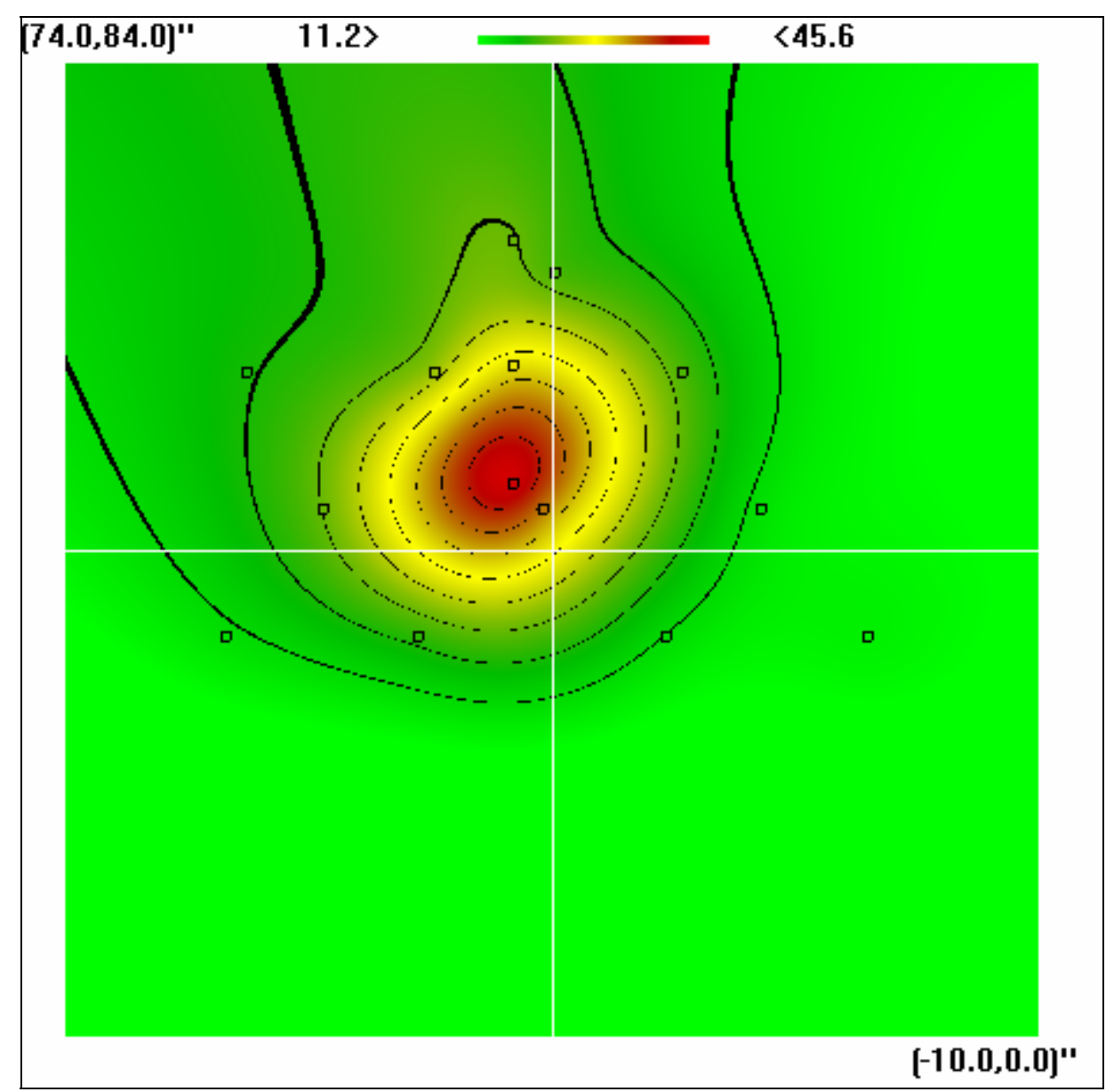

Figure 98 Temperature Mapping Results for an Axial Placement of $6 \mathrm{ft}$ (Taken on Day 5).

(Color Scale Refers to Temp. (C)) 


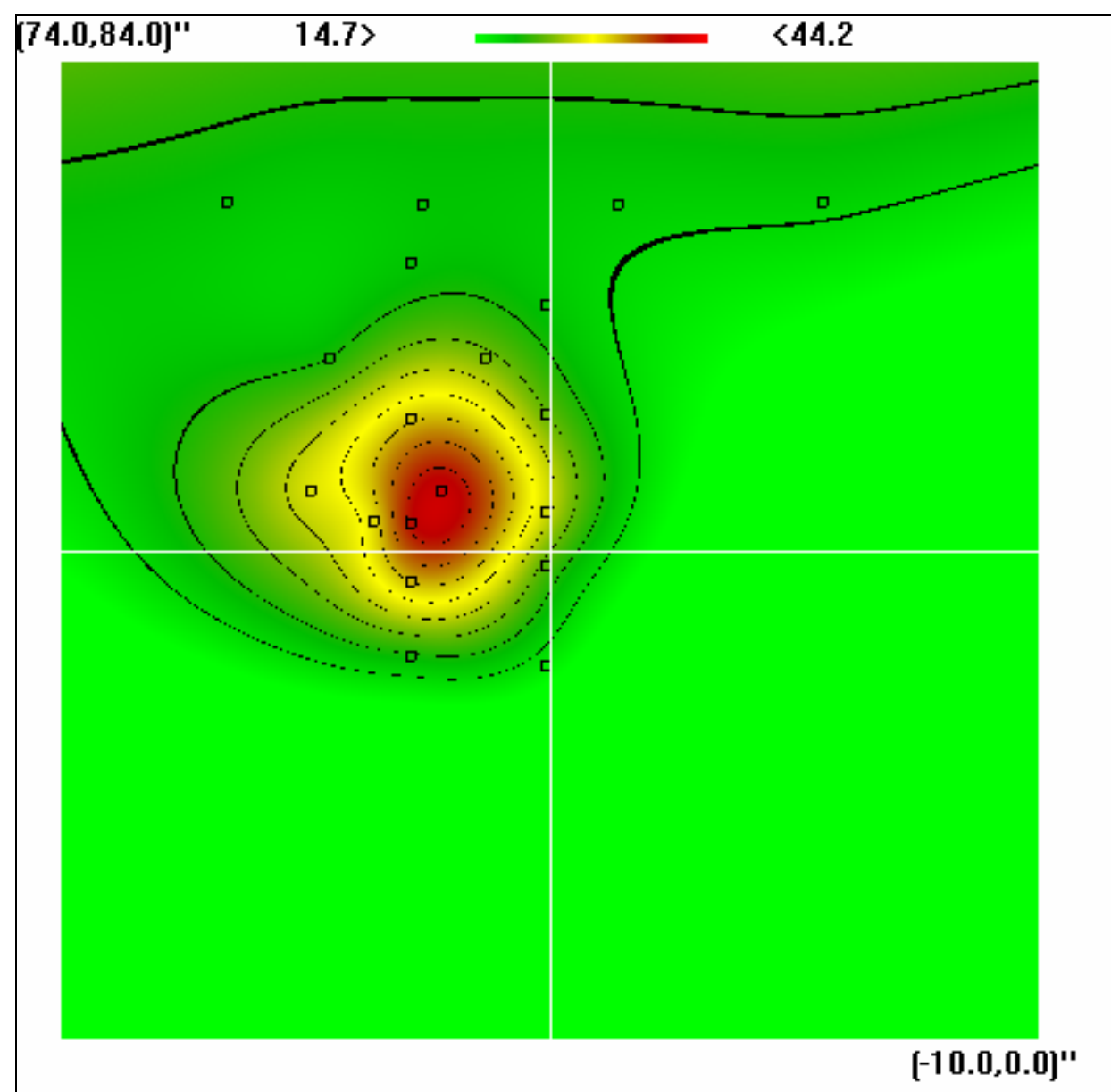

Figure 99 Temperature Mapping Results for an Axial Placement of $6 \mathrm{ft}$ (Taken on Day 6).

(Color Scale Refers to Temp. (C)) 


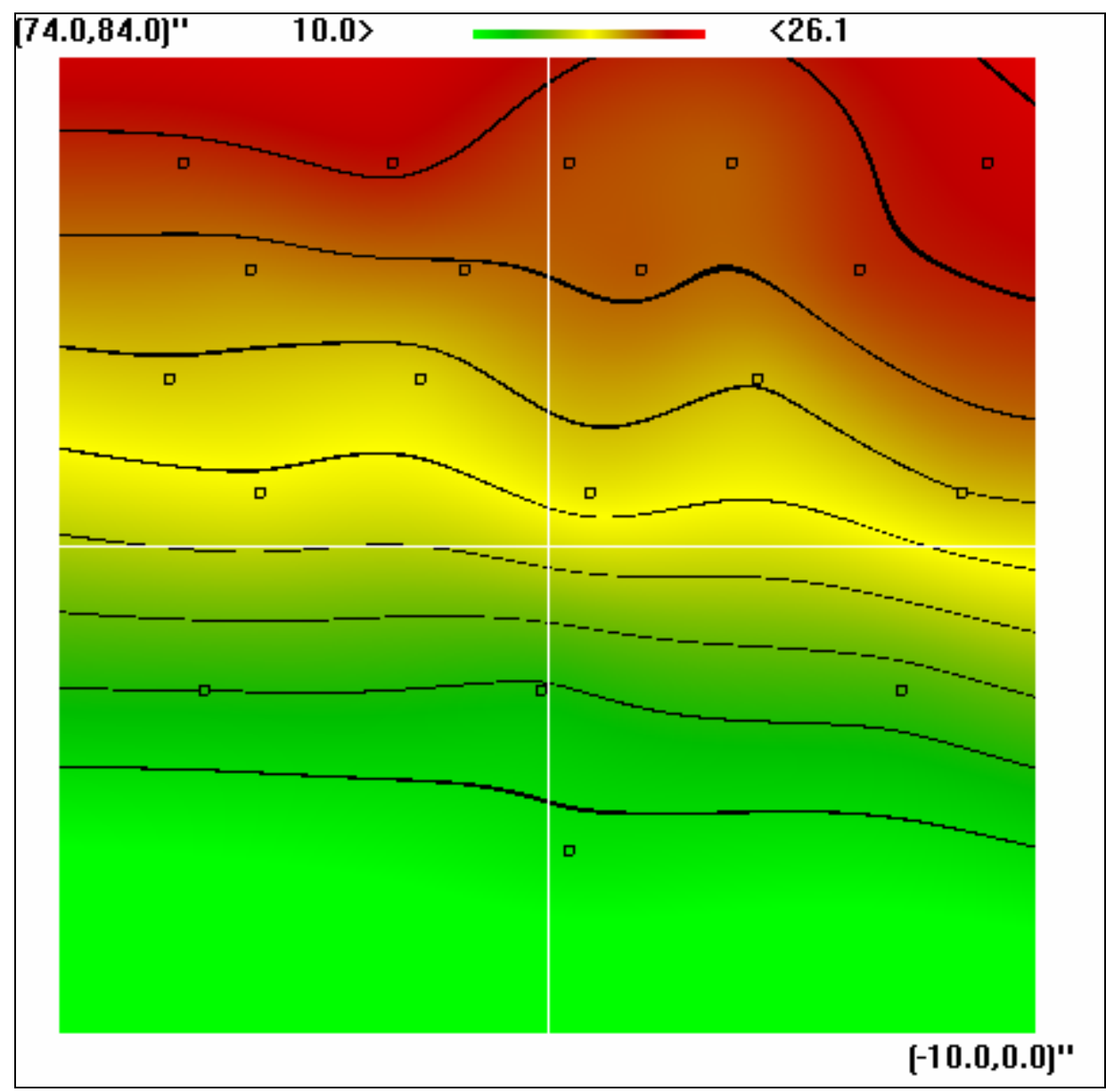

Figure 100 Temperature Mapping Results for an Axial Placement of $20 \mathrm{ft}$ (Taken on Day 5).

(Color Scale Refers to Temp. (C)) 


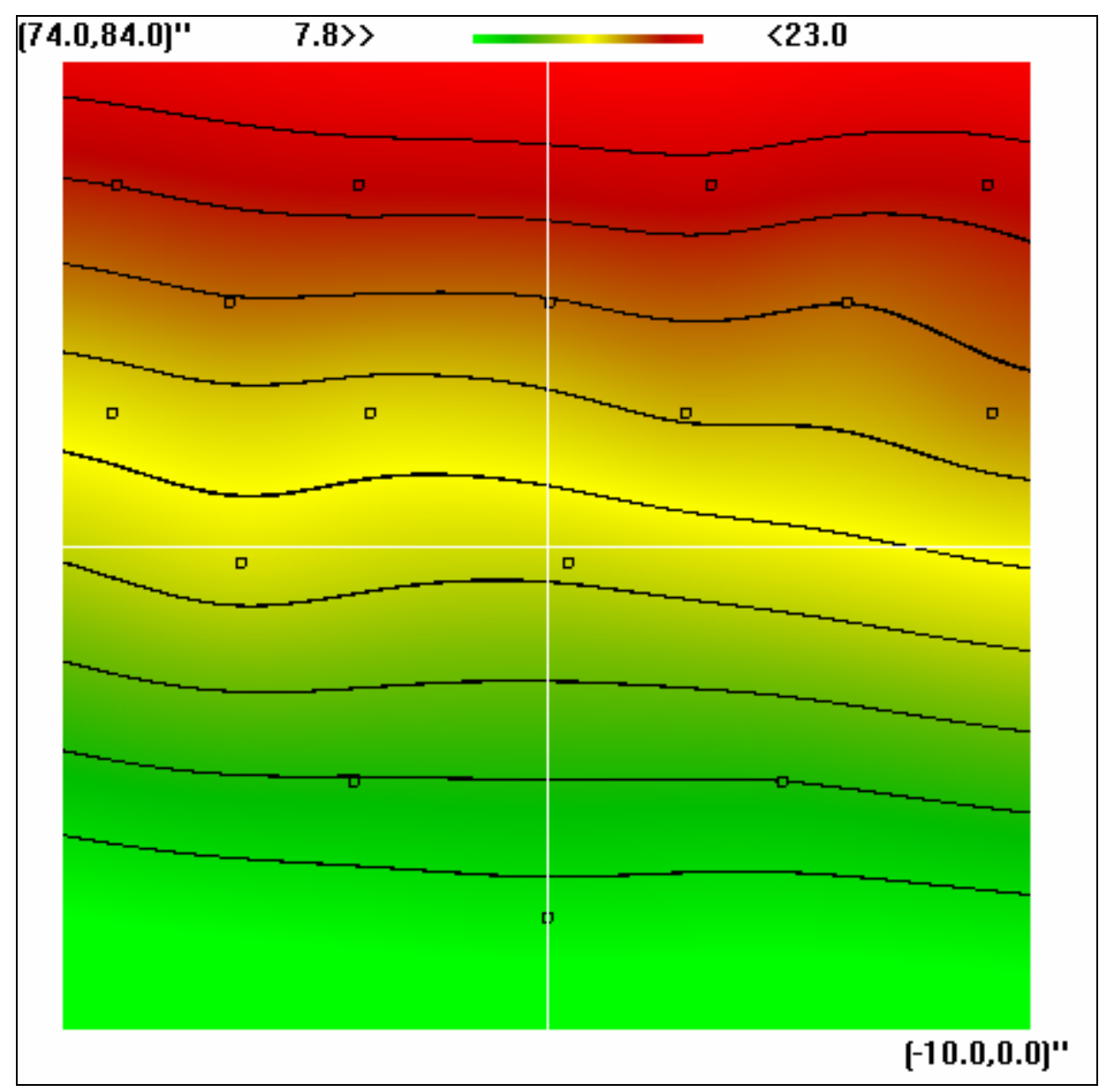

Figure 101 Temperature Mapping Results for an Axial Placement of $20 \mathrm{ft}$ (Taken on Day 6).

(Color Scale Refers to Temp. (C)) 


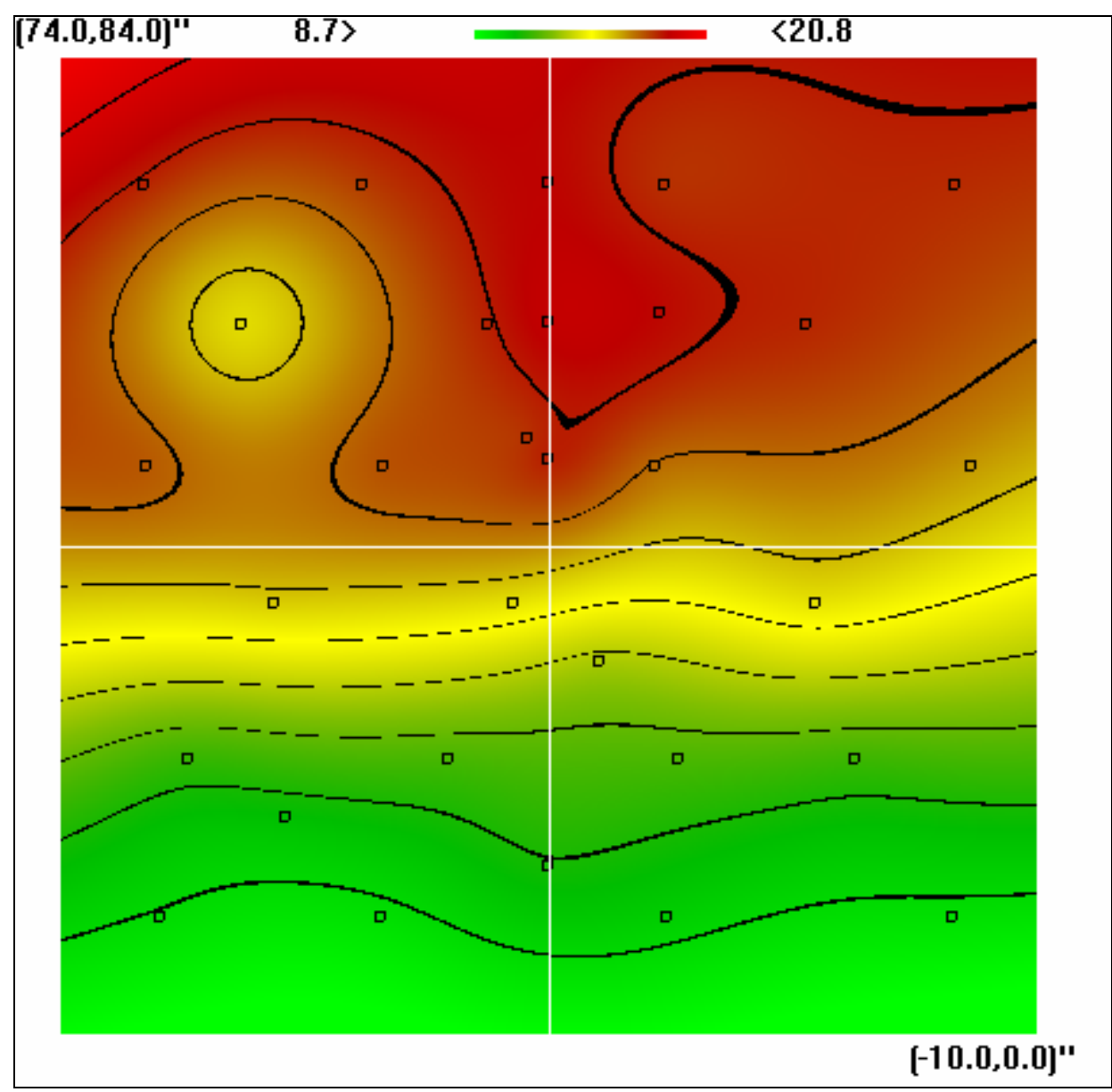

Figure 102 Temperature Mapping Results for an Axial Placement of $50 \mathrm{ft}$ (Taken on Day 7).

(Color Scale Refers to Temp. (C)) 


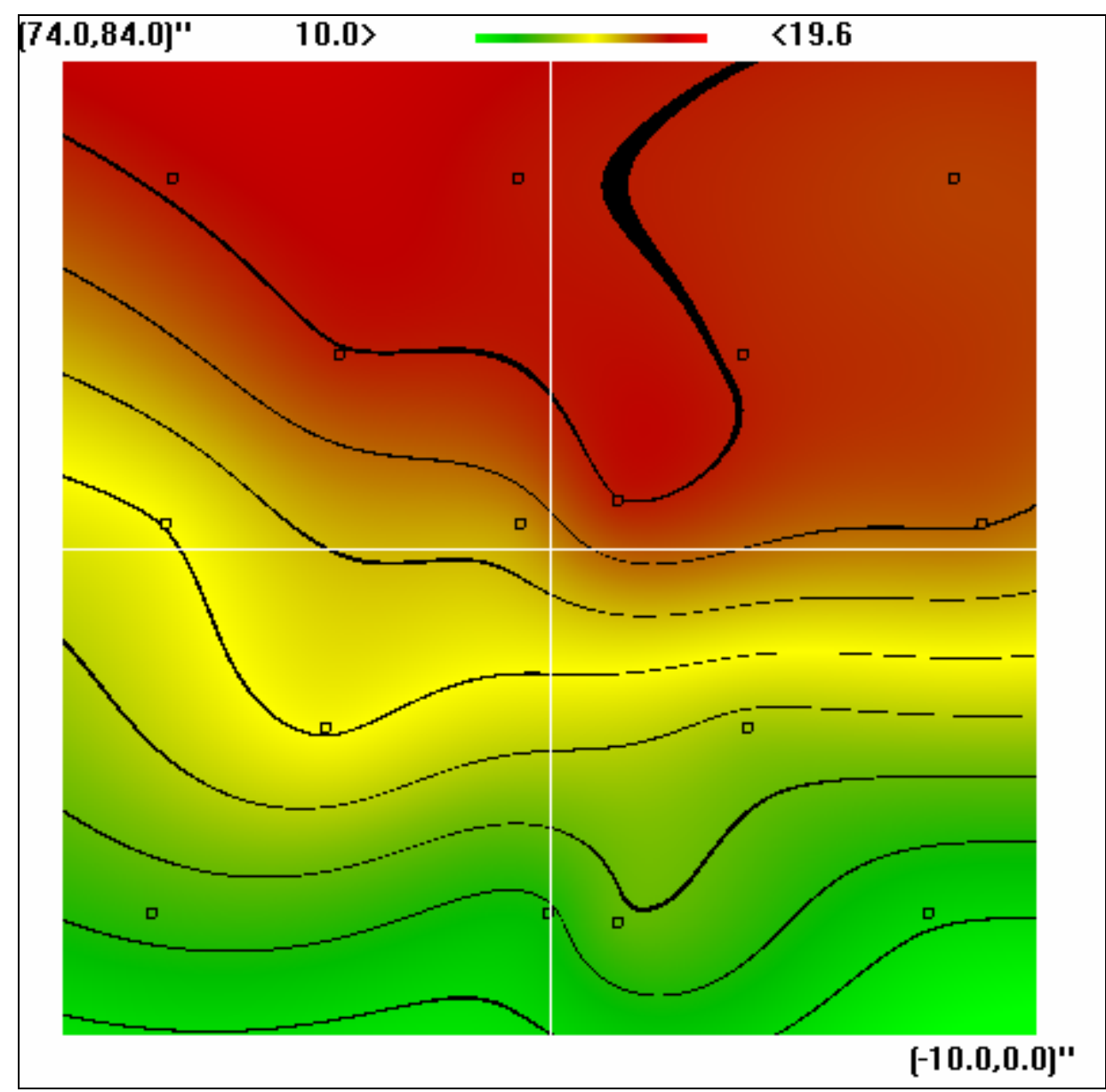

Figure 103 Temperature Mapping Results for an Axial Placement of $125 \mathrm{ft}$ (Taken on Day 5). (Color Scale Refers to Temp. (C)) 


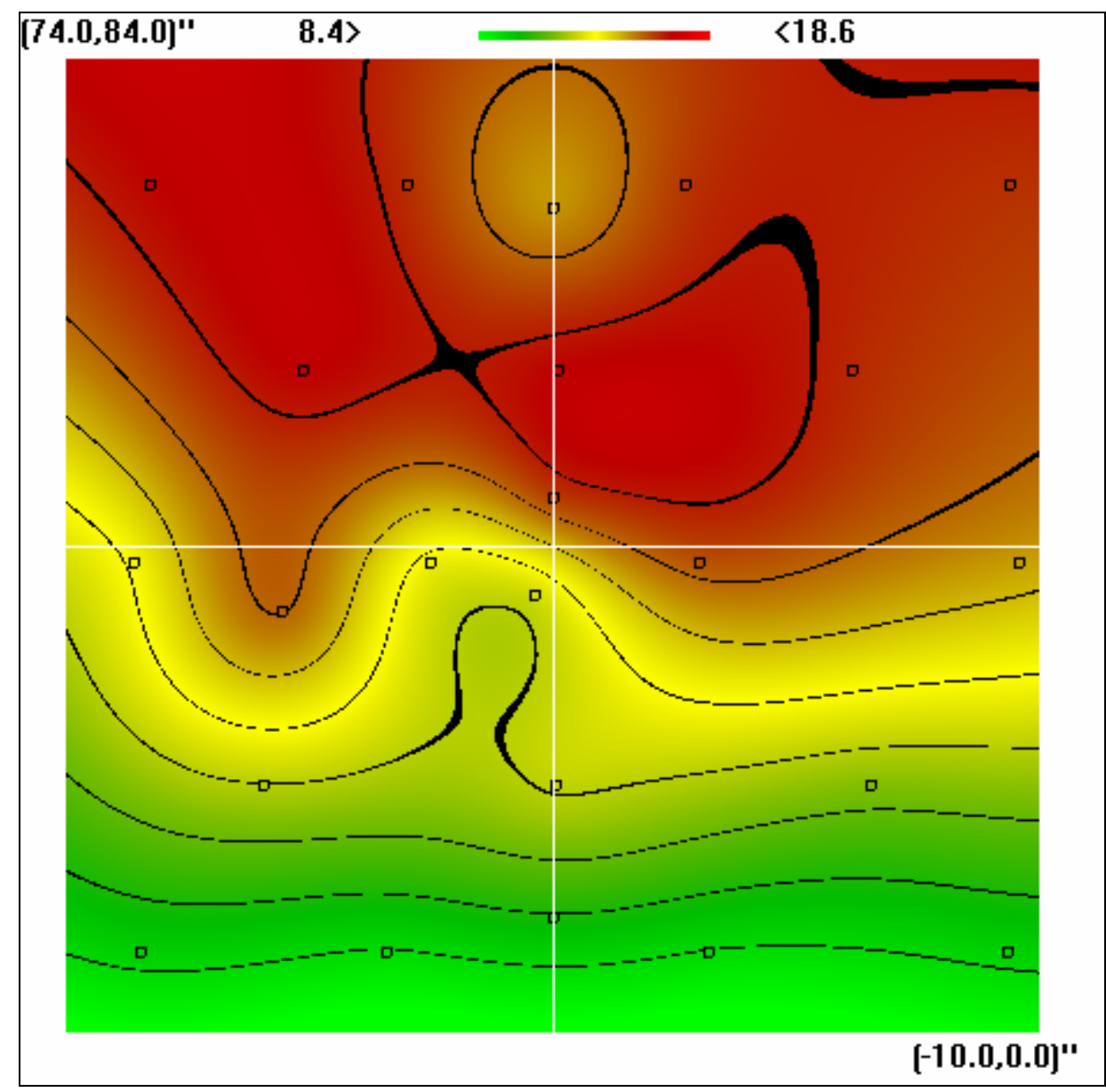

Figure 104 Temperature Mapping Results for an Axial Placement of $125 \mathrm{ft}$ (Taken on Day 6). (Color Scale Refers to Temp. (C)) 


\section{Appendix B (130 Temperature Maps)}

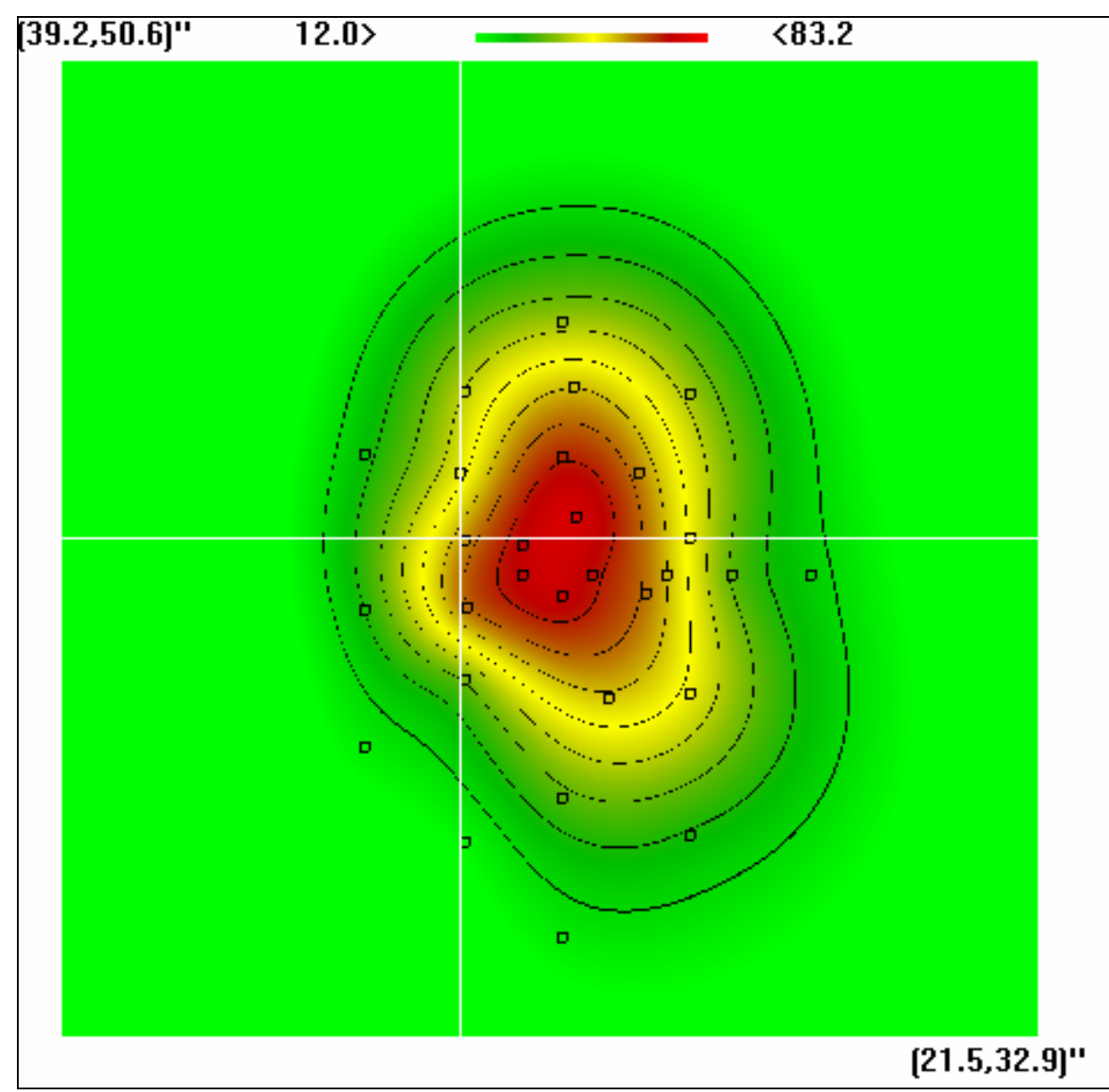

Figure 105 Temperature Mapping Results for an Axial Placement of 11 inches (Taken on Day 10).

(Color Scale Refers to Temp. (C)) 


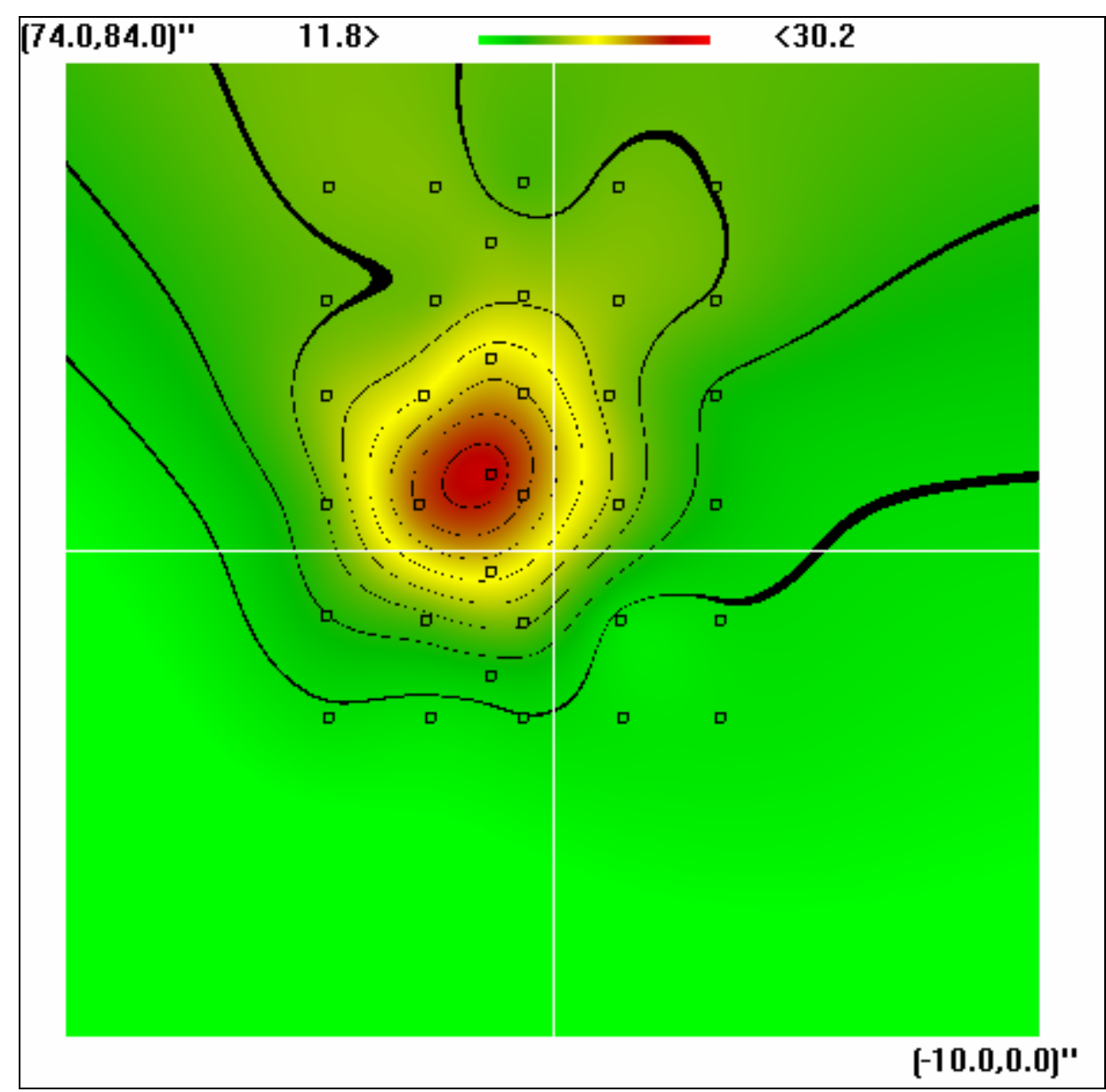

Figure 106 Temperature Mapping Results for an Axial Placement of $6 \mathrm{ft}$ (Taken on Day 8).

(Color Scale Refers to Temp. (C)) 


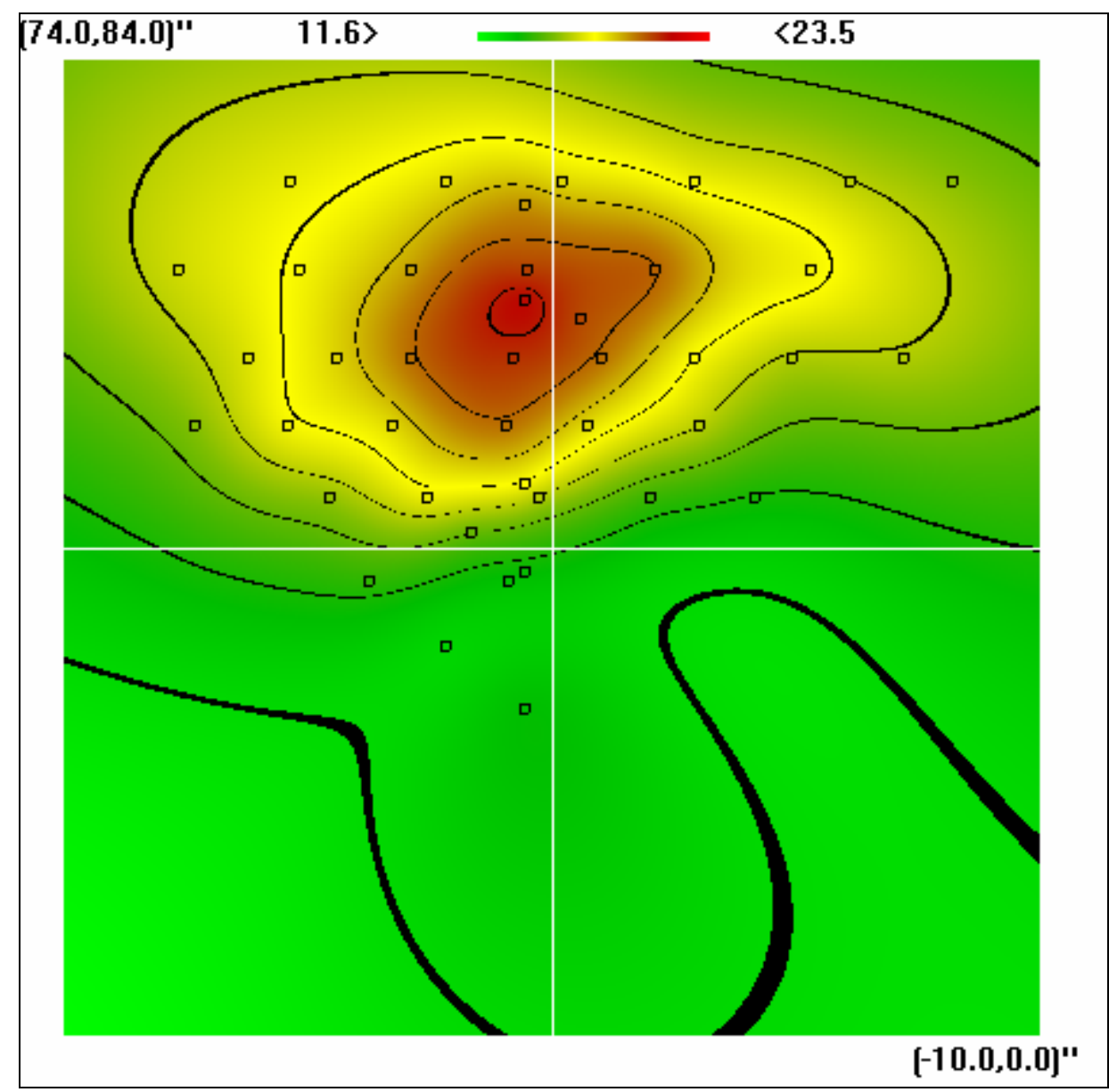

Figure 107 Temperature Mapping Results for an Axial Placement of $10 \mathrm{ft}$ (Taken on Day 8).

(Color Scale Refers to Temp. (C)) 


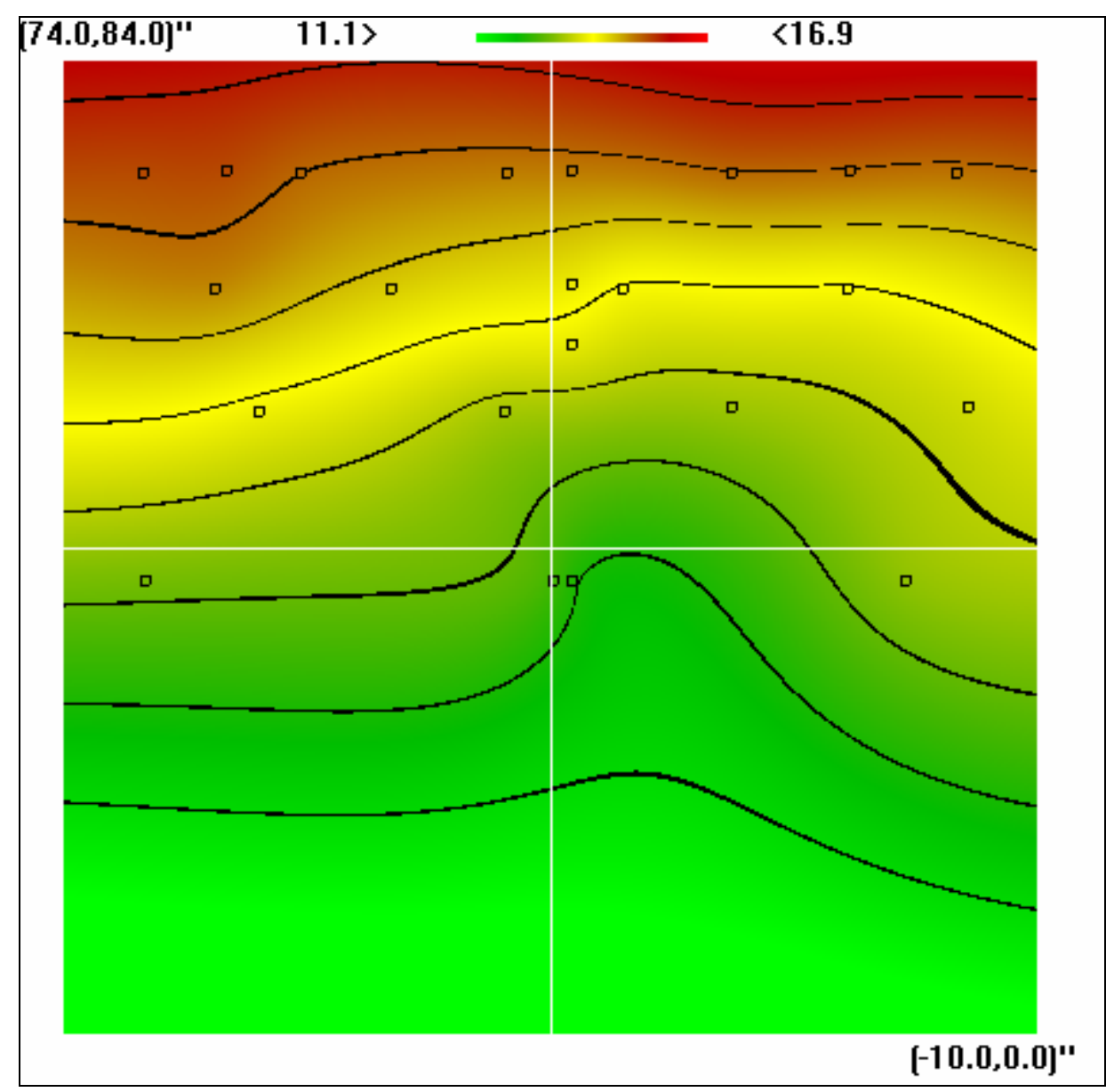

Figure 108 Temperature Mapping Results for an Axial Placement of $25 \mathrm{ft}$ (Taken on Day 10).

(Color Scale Refers to Temp. (C)) 


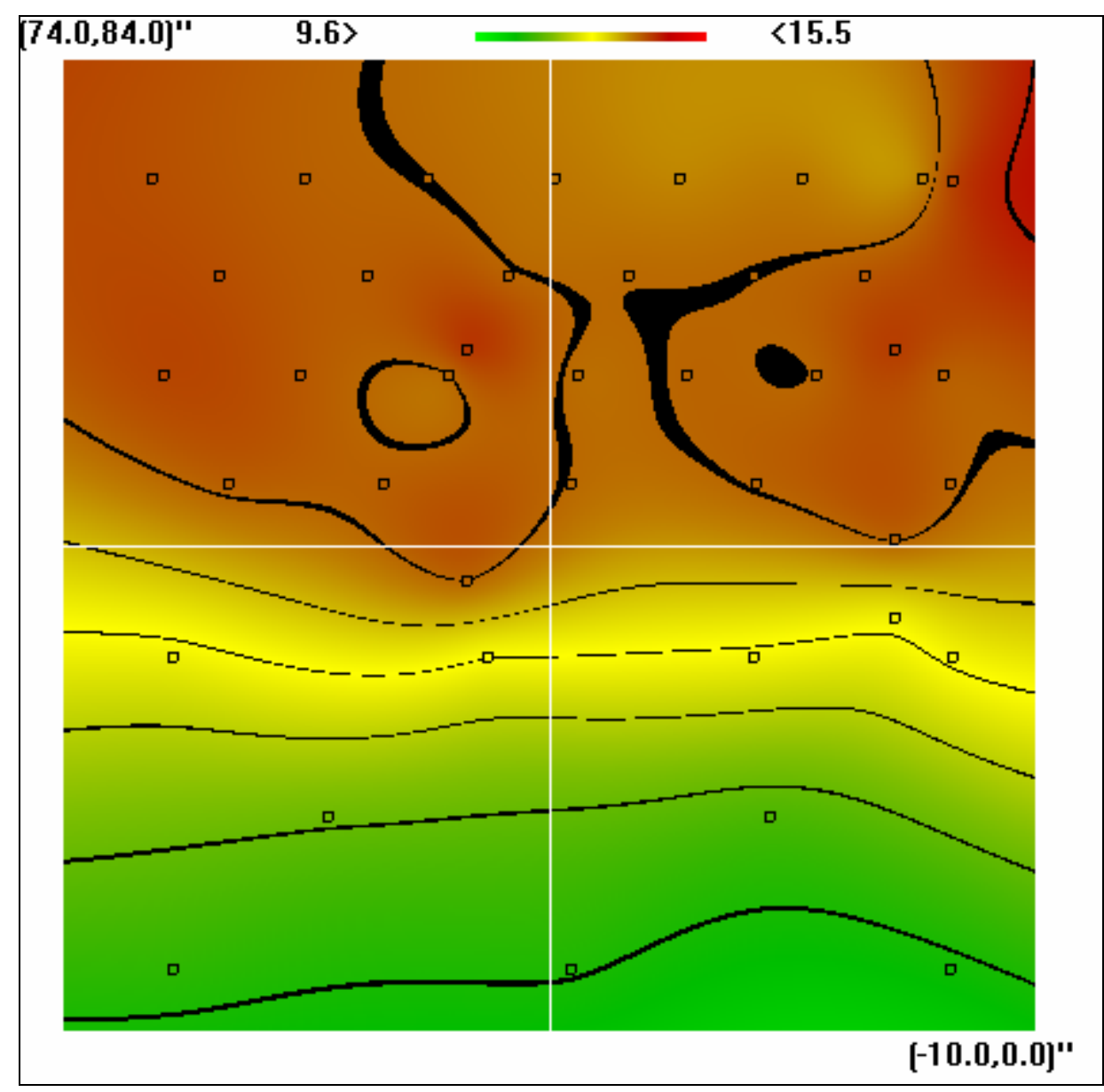

Figure 109 Temperature Mapping Results for an Axial Placement of $100 \mathrm{ft}$ (Taken on Day 10). (Color Scale Refers to Temp. (C)) 


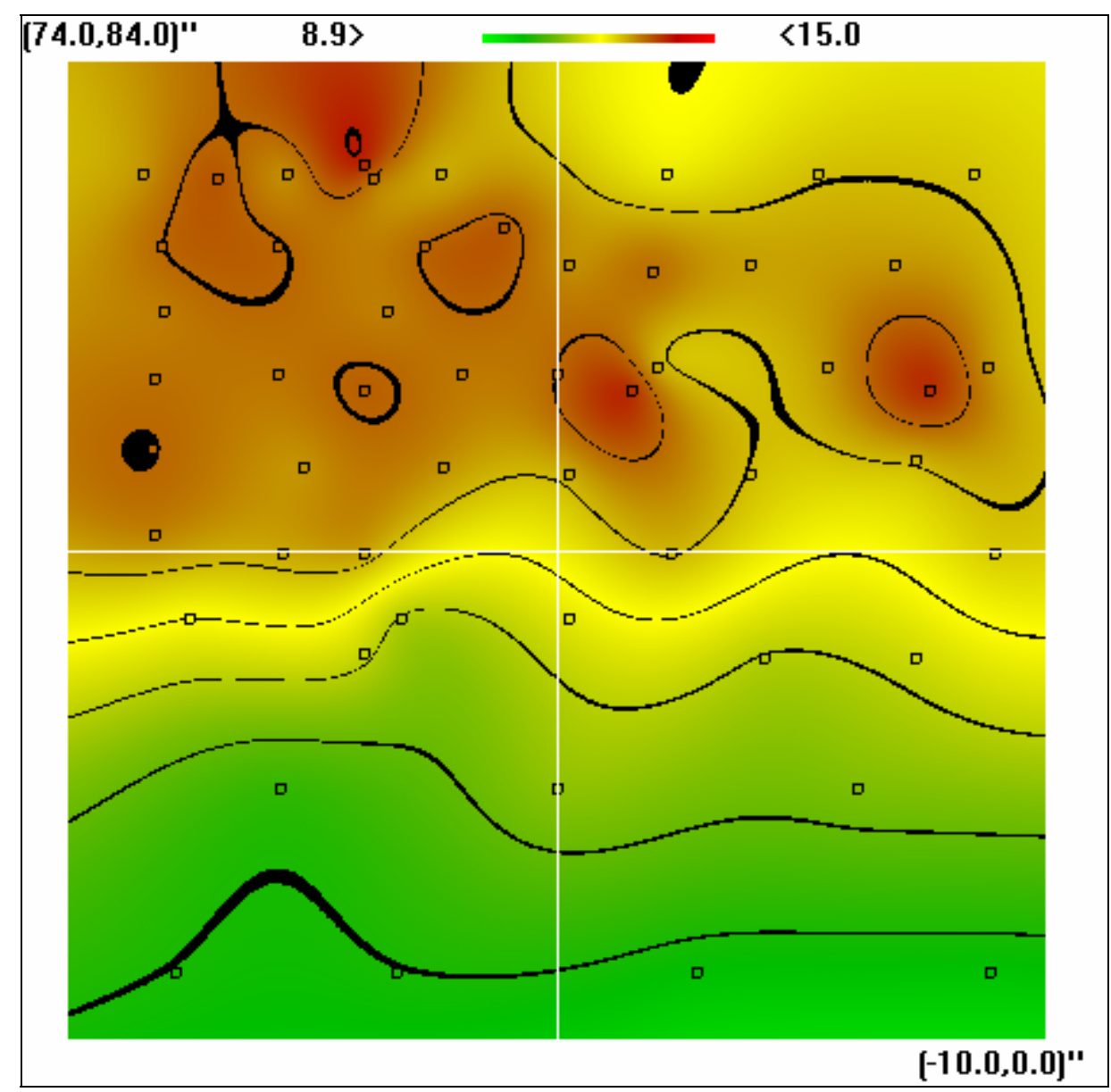

Figure 110 Temperature Mapping Results for an Axial Placement of $125 \mathrm{ft}$ (Taken on Day 9).

(Color Scale Refers to Temp. (C)) 


\section{Appendix C (Mapping Code Sample)}

\#include <windows.h>

\#include <math.h>

\#include $<$ WINGDI.h>

\#include <stdio.h>

static HWND hwnd;

int ord;

int nn;

int value,valred,valgreen,valblue;

float fvalue;

int ii,jj,kk,jj,m;

int xyzint[200][3];

float $x y z[200][4]$;

static float $z \min , z \max , x \min =-10, x \max =74, y \min =0, y \max =84, z$ scale $=1$,

axialmin $=140$, axialmax $=240$;

static int numCuts $=45$, cyClientsize $=300$;

static float zpercut $=0.25$, axialstretch $=2$, thick $=.01 ; / /$ all values greater

//static float zmin,zmax, xmin=-

10, $x \max =74, y \min =0, y \max =84, z$ scale $=1$, axialmin $=36$, axialmax $=120$;

//static float zmin,zmax, $x \min =-10, x \max =74, y \min =11, y \max =22.7, z$ scale $=0$;

static float

zoomrad $=42$, zoomcenter $x=32$, zoomcentery $=42$, alpha $=0$, beta $=0$,zeta $=0$;

float radius[81],zcalc,sumrad;

float coeffxy[200][200],constxy[200],coeffsol[200];

FILE *fp, ${ }^{\star} \mathrm{fr}$;

static int cxClient, cyClient ;

static HDC hdc=NULL;

static PAINTSTRUCT ps ;

static int togglexy=-1;

static int oldii,oldjj;

static COLORREF

oldcolor[4][4],oldcolorPM[4][4],bmap[3500][3500];

static int initializedbmap $=0$;

static float transmatrix[3][4],ztest;

static int sweepy $=1$, sweep $=1$;

static float zperbmap[3500][3500];

static int PMoffset=25;

COLORREF oldColorTest;

int decx,decy,decz;

void calc ()\{

float $x, y, a x i a l, z, x y, c, x o, y o, S i, r i ;$

int a,b,aa,bb;

int test,ee,ff;

fr=fopen("Summary_xyz.dat","r");

fscanf(fr,"\%i",\&nn);

fscanf(fr,"\%i",\&ord);//not used any more

for $(e e=0 ; e e<n n ; e e++)\{$

fscanf(fr, "\%f",\&c); 


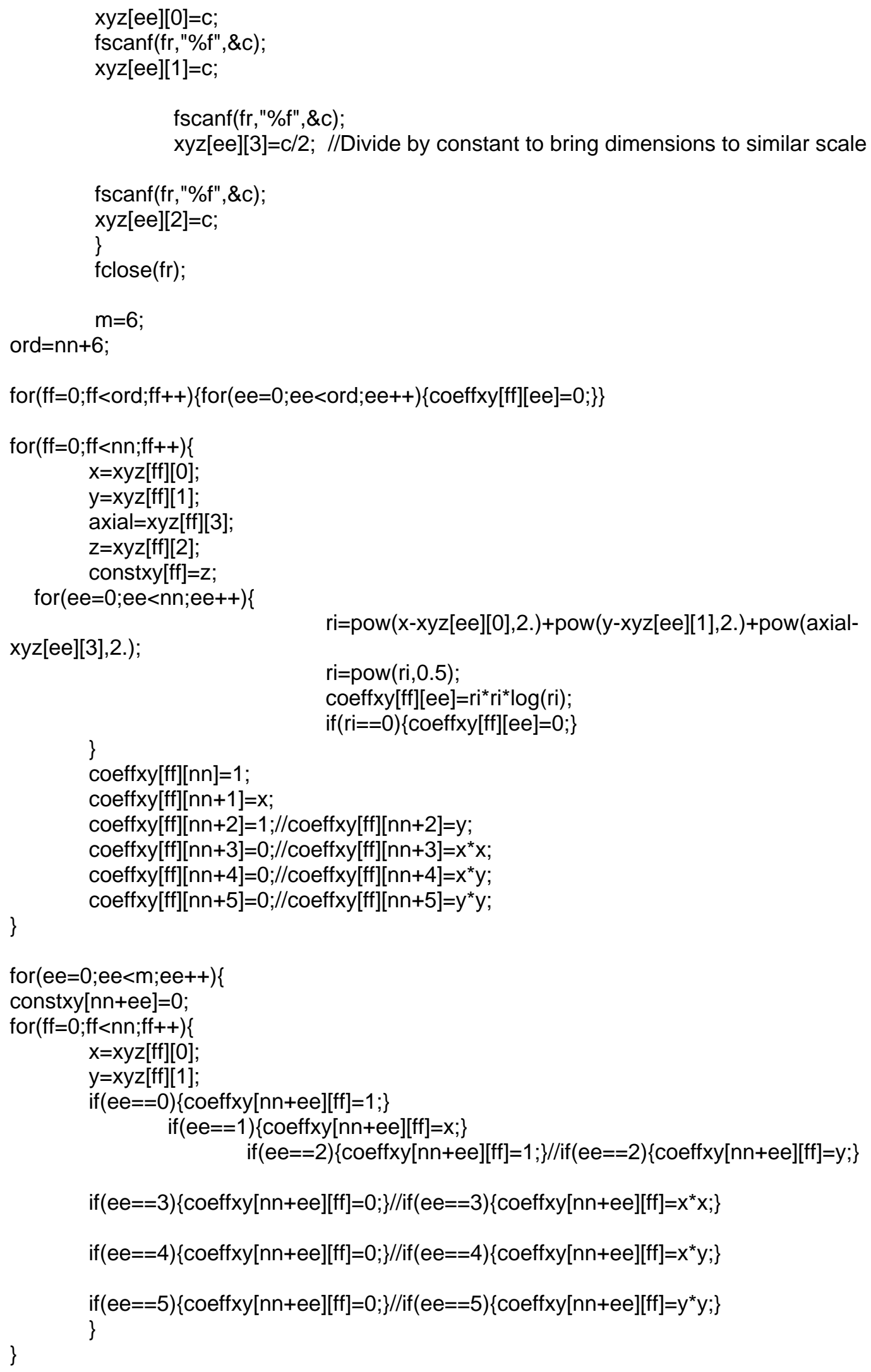


for(ee=0;ee<ord;ee++)\{

if(coeffxy[ee][ee] $==0)\{$

for(ff=ee+1;ff<ord;ff++)\{if(coeffxy[ff][ee]!=0)\{for(kk=ee;kk<ord;kk++)\{c=coeffxy[ff][kk];coeffxy[ff][kk]

$=$ coeffxy[ee][kk];coeffxy[ee][kk]=c;\}c=constxy[ee];constxy[ee]=const $x y[f] ;$ constxy[ff] $=c ; f f=o r d+2 ;\}$

$c=$ coeffxy[ee][ee];

\}

if $(\mathrm{c} !=0)\{$

for $(f f=e e ; f f<o r d ; f f++)\{c o e f f x y[e e][f f]=c o e f f x y[e e][f f] / c ;\} \operatorname{constxy}[e e]=$ constxy[ee]/c;

for(ff=ee+1;ff<ord;ff++)\{c=coeffxy[ff][ee];for(kk=ee;kk<ord;kk++)\{coeffxy[ff] $[k k]=$ coeffxy[ff] $\left.k k]-c^{*} \operatorname{coeffxy}[\mathrm{ee}][\mathrm{kk}] ;\right\}$ constxy[ff] $=$ constxy[ff]-c*constxy[ee];\}

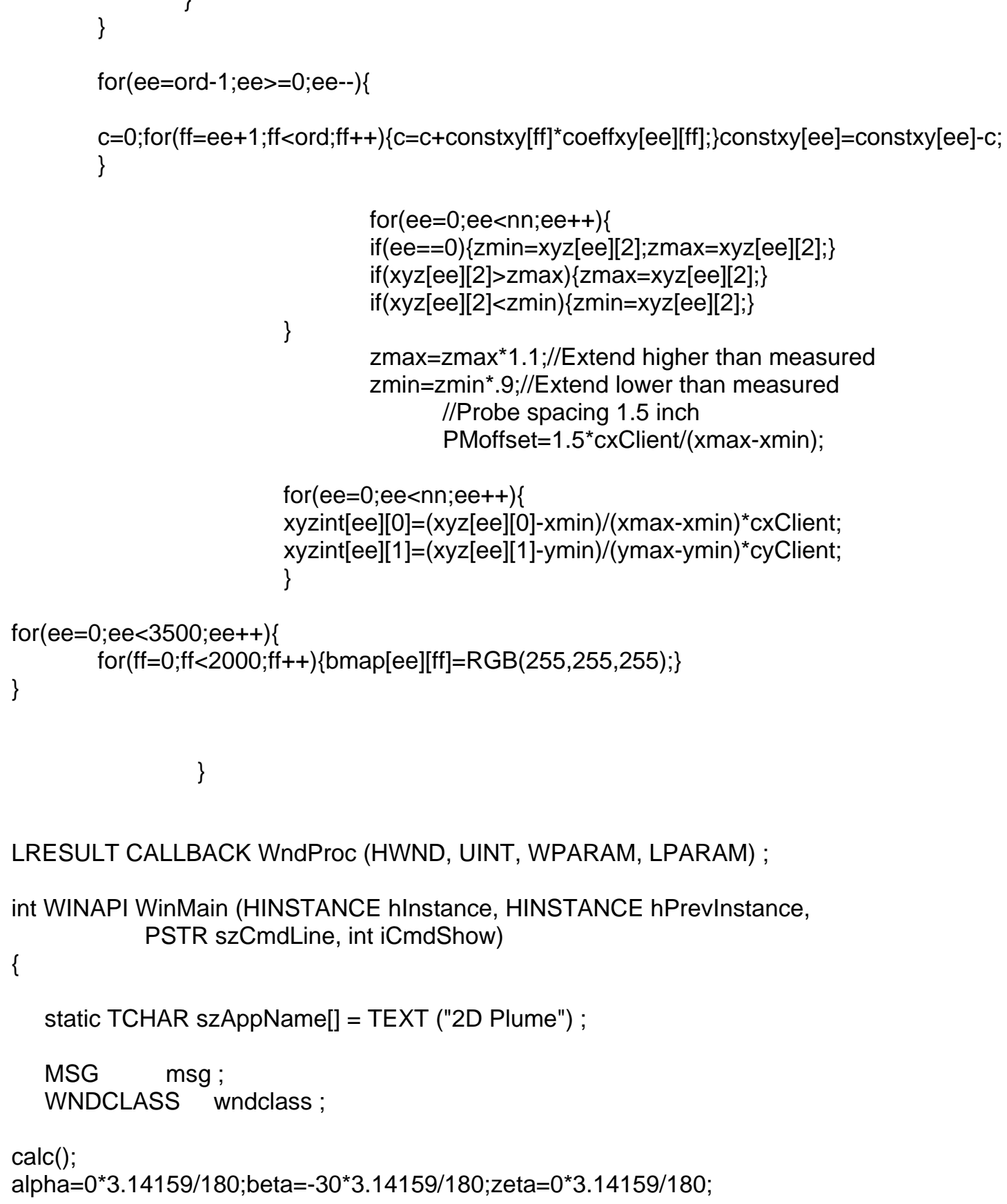




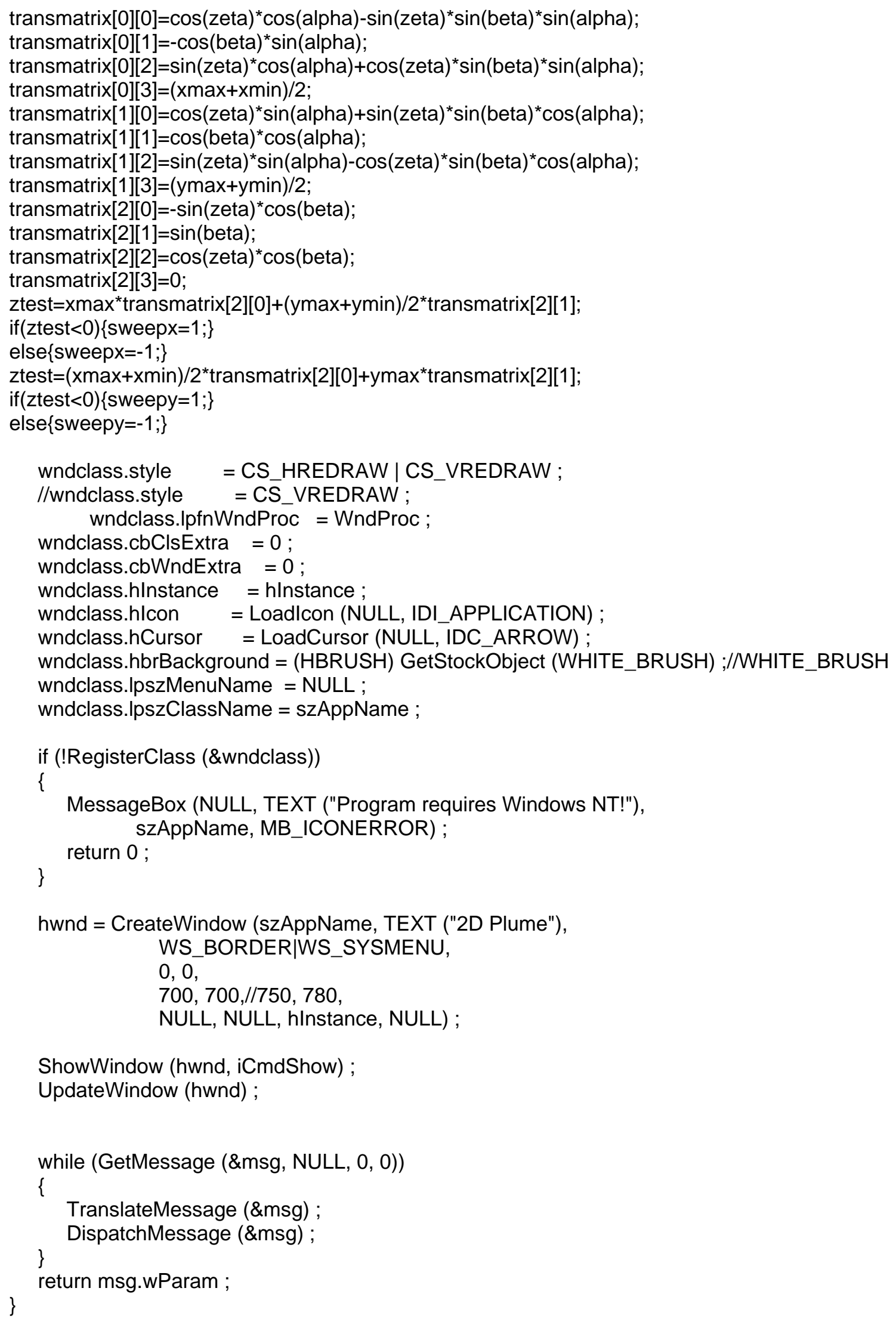


LRESULT CALLBACK WndProc (HWND hwnd, UINT message, WPARAM wParam, LPARAM IParam)

\{

//static HDC hdc;

int $\mathrm{i}$;

//static PAINTSTRUCT ps ;

POINT apt [NUM] ;

float xper,yper,zper,x,y,axial,z,valyellow,az,bz,cz,dz,ez,fz,ri,xp,yp;

int test,jjj,mod,dim,dimx,dimy,col,ylow,yup,xlow,xup,axialsweep,sint;

int ptwidth=2,st,stt;

TCHAR szBuffer[60];

char cszBuffer[60];

int sj,bj,cj, si,bi,ci;

float xdisplay,ydisplay;

int tranii,tranjj,transcale $=1$;

//cyClient=300;

cyClient=cyClientsize;

//cxClient=200;//cyClient*(axialmax-axialmin)/(ymax-ymin);

cxClient=cyClient ${ }^{\star}($ axialmax-axialmin $) /(y \max -y \min )$;

transcale $=2$;

remove transformation holes.

//If display is reduced memory reduces and transcale can increase to

switch (message)

\{

case WM_RBUTTONDOWN://Place a reference dot

st=LOWORD(IParam);

stt=HIWORD(IParam);

for $\left(k \mathrm{k}=0 ; \mathrm{kk}<2^{\star} 2 ; \mathrm{kk}++\right)\{$ for $(\mathrm{jjj}=-2 ; \mathrm{jjj}<=2 ; \mathrm{jjj}++)\{$

SetPixel(hdc,st-2+kk,stt+jjj, RGB(0,0,200));\}\}

return 0;

case WM_LBUTTONDOWN:

if $(($ wParam \&\& MK_LBUTTON $)==1)\{$

st=LOWORD(IParam);

stt=HIWORD(IParam);

if(LOWORD $($ IParam $)<440)\{$

zoomcenterx $=\mathrm{x}$;

zoomcentery $=\mathrm{y}$;

TextOut(hdc,445,25,szBuffer,wsprintf(szBuffer,TEXT("a+"))); for $(\mathrm{kk}=0 ; \mathrm{kk}<33 ; \mathrm{kk}++)\{$ SetPixel(hdc,kk+440,25, RGB $(0,0,0)) ;\}$ for $(k \mathrm{k}=0 ; \mathrm{kk}<33 ; \mathrm{kk}++)\{$ SetPixel(hdc,kk+440,40,RGB(0,0,0)); TextOut(hdc,445,75,szBuffer,wsprintf(szBuffer,TEXT("b+"))); for $(\mathrm{kk}=0 ; \mathrm{kk}<33 ; \mathrm{kk}++)\{$ SetPixel(hdc,kk+440,75, RGB $(0,0,0)) ;\}$ for $(\mathrm{kk}=0 ; \mathrm{kk}<33 ; \mathrm{kk}++)\{$ SetPixel(hdc,kk+440,90,RGB $(0,0,0)) ;\}$ TextOut(hdc,445,125,szBuffer,wsprintf(szBuffer,TEXT("c+"))); for $(k \mathrm{k}=0 ; \mathrm{kk}<33 ; \mathrm{kk++})\{$ SetPixel(hdc, $\mathrm{kk}+440,125, \operatorname{RGB}(0,0,0)) ;\}$ for $(k \mathrm{k}=0 ; \mathrm{kk}<33 ; \mathrm{kk++})\{$ SetPixel(hdc, $\mathrm{kk}+440,140, \operatorname{RGB}(0,0,0)) ;\}$ \} 


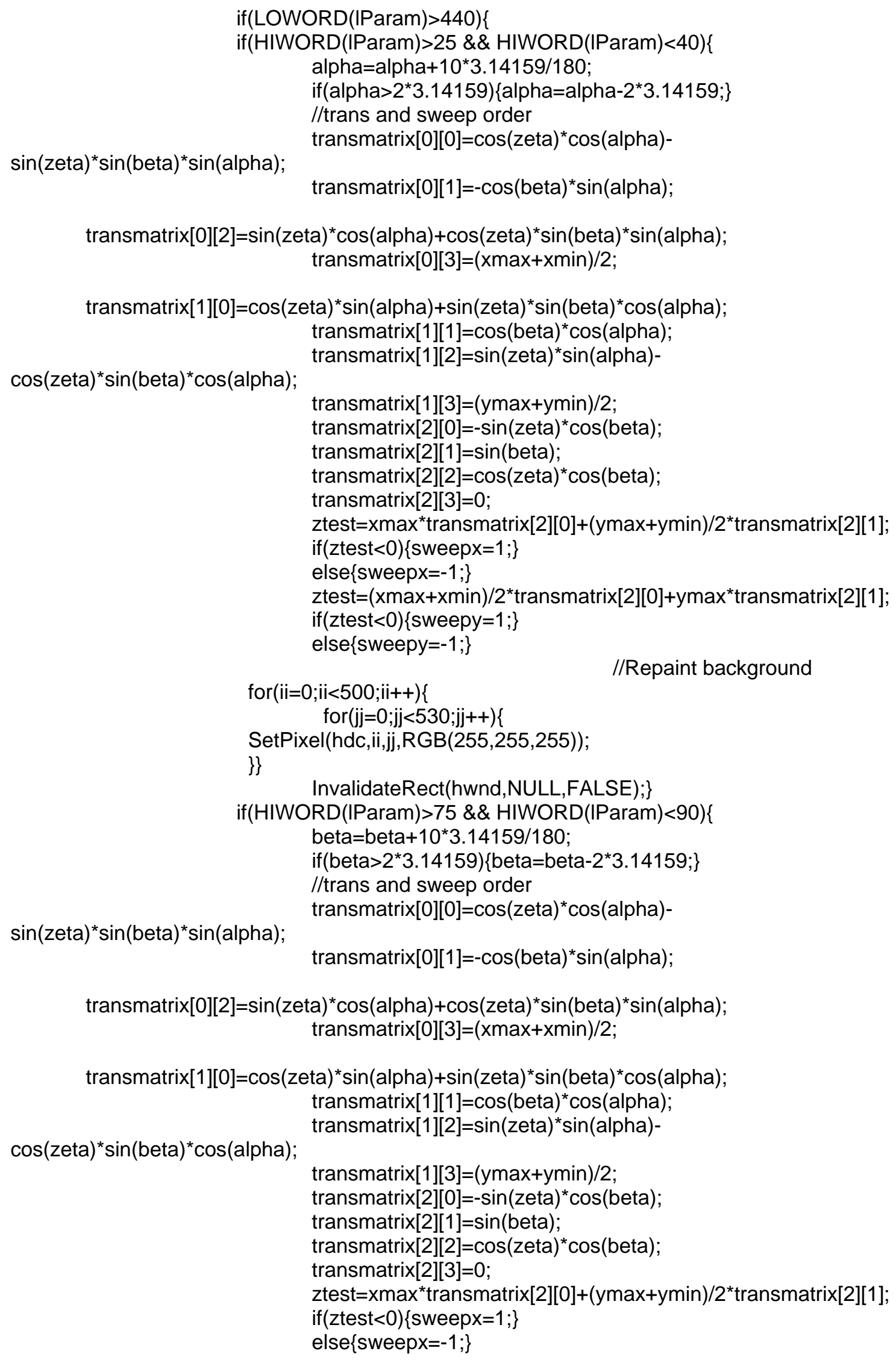




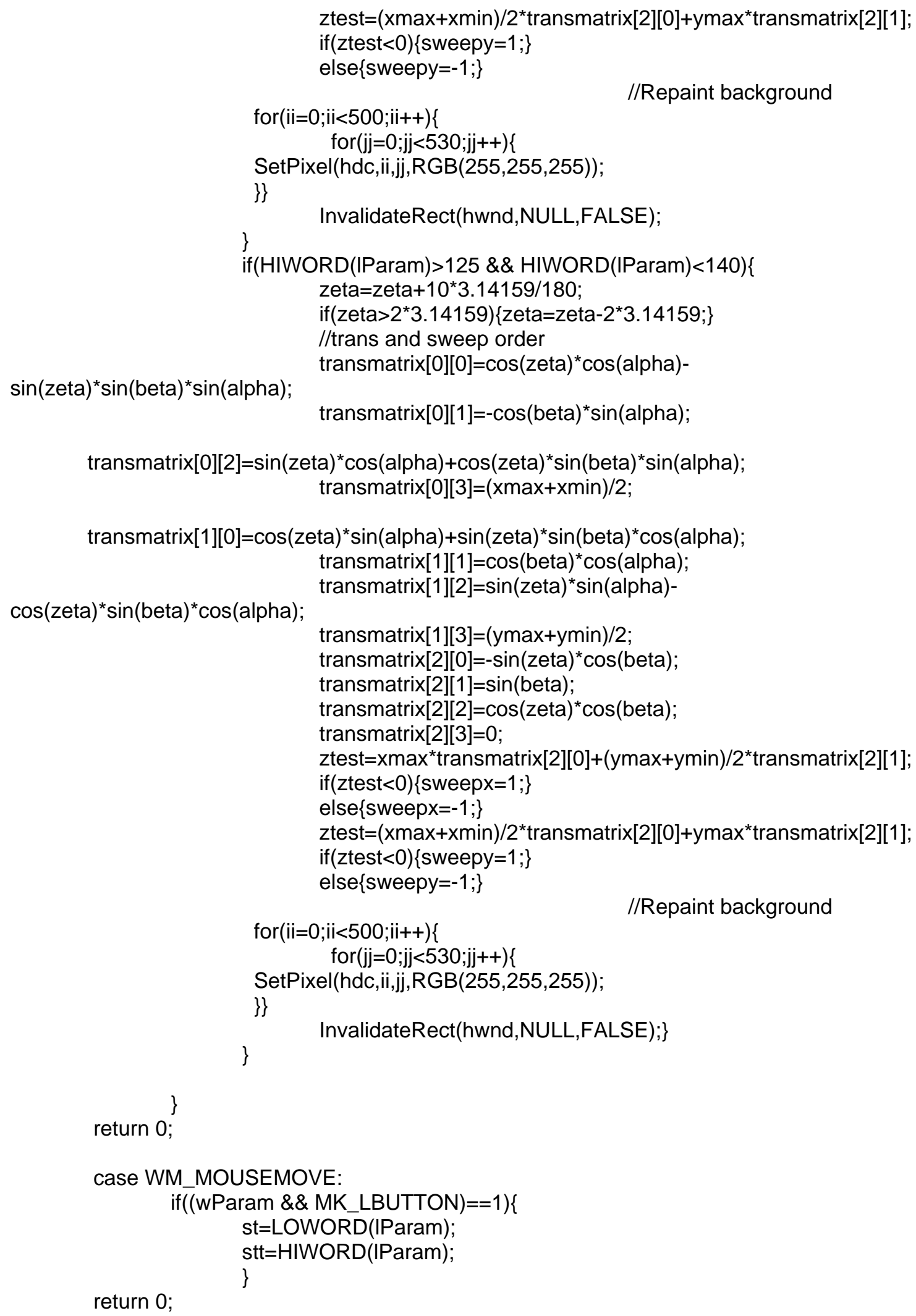


case WM_USER + 757: //WPARAM can not be negative so must distinguish by message

$$
\begin{aligned}
& \text { if(togglexy==1)\{ } \\
& \qquad \begin{aligned}
\text { for }(k \mathrm{k}=0 ; & ; \mathrm{kk}<2 * 2 ; \mathrm{kk}++)\{\text { for(jijj=-2;jjj< }=2 ; \mathrm{jjj}++)\{ \\
& \text { SetPixel(hdc,oldii+20-2+kk,oldjj+20+jjj, oldcolor[kk][jjj+2]); } \\
& \text { SetPixel(hdc, oldii+20-2+kk- }
\end{aligned}
\end{aligned}
$$

PMoffset,oldjj+20+jjj,oldcolorPM[kk][ijj+2]);

\}\}

\}

$$
\text { if }(\text { togglexy==-1)\{ }
$$

$\mathrm{x}=$ (float)wParam;

$\mathrm{y}=$ (float)IParam;

$/ / x$ and $y$ are sent as integer number of tenths 0.1 by dll

$\mathrm{x}=\mathrm{x}^{\star} 0.1$;

$\mathrm{y}=\mathrm{y}^{\star} 0.1$

can.

$\mathrm{x}=-\mathrm{x} ; / / 757$ means $\mathrm{x}$ is negative but WPARAM can not be while LPARAM

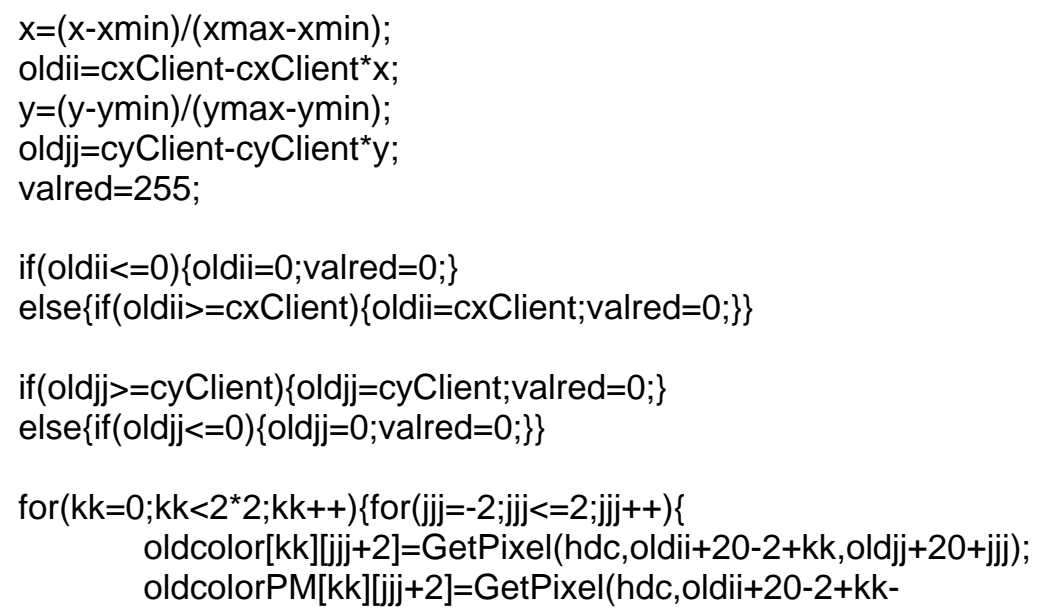

PMoffset,oldjj+20+jjj);

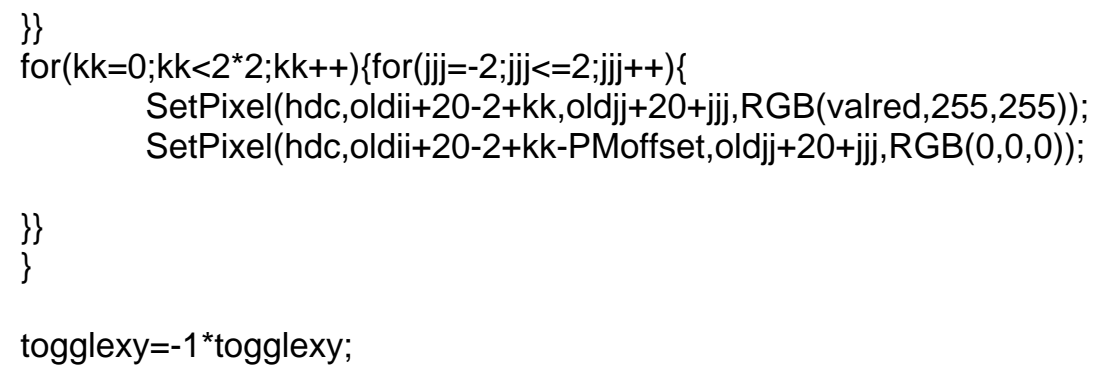

return 0;

case WM_USER + 755: I/Measurexy is sending xy coordinate to be projected

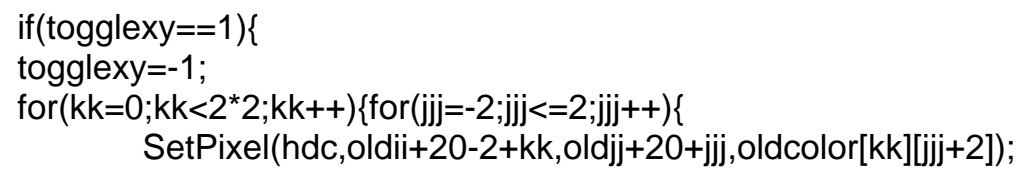


SetPixel(hdc,oldii+20-2+kk-

PMoffset,oldjj+20+jjj, oldcolorPM[kk][jjj+2]);

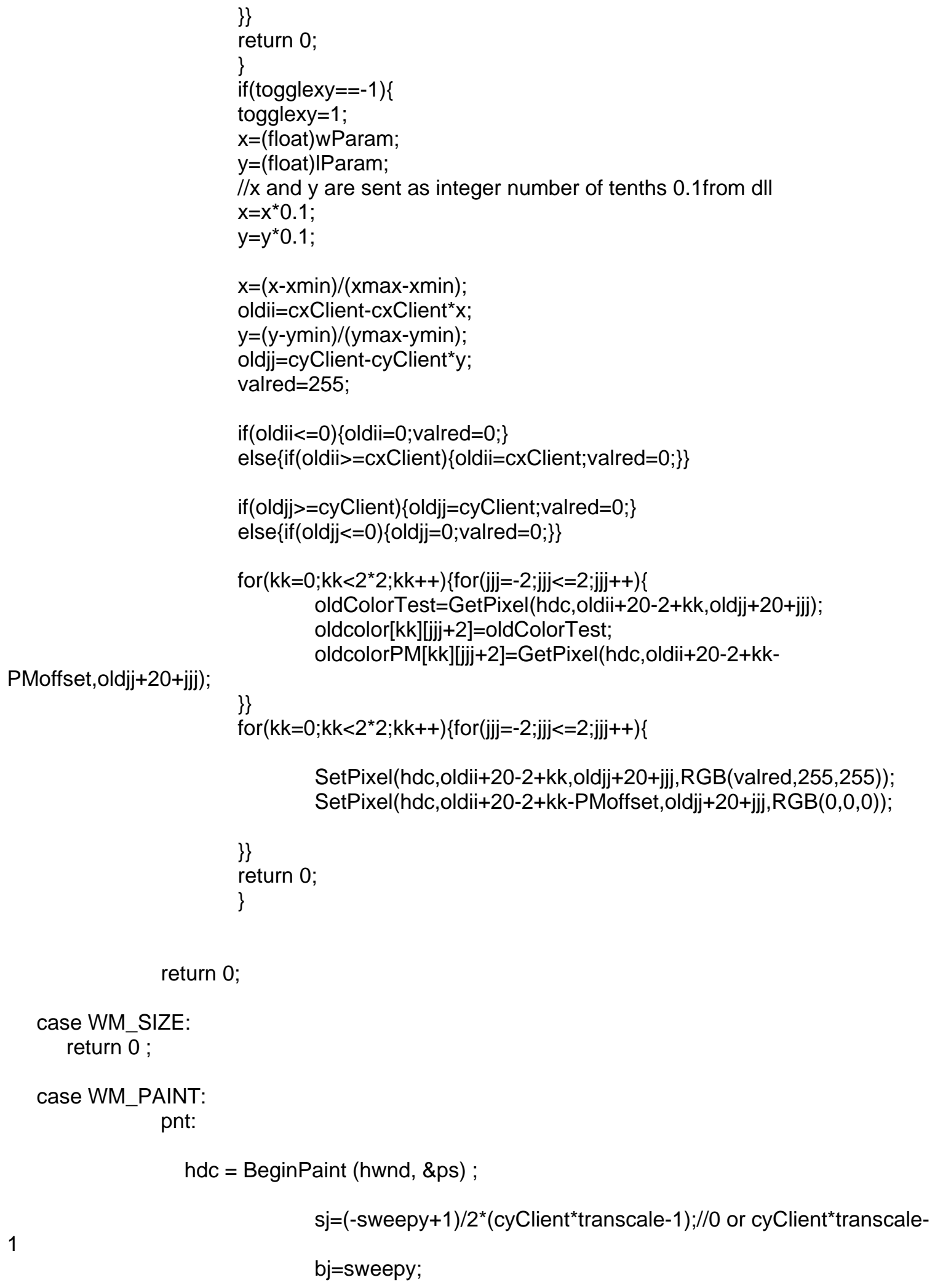


cj=(sweepy-1)/2;

1

$\mathrm{si}=(-$ sweepx +1$) / 2^{\star}\left(\mathrm{cxClient}{ }^{\star}\right.$ transcale-1);//0 or cxClient*transcale-

bi=sweepx;

$\mathrm{ci}=($ sweepx-1)/2;

for(axialsweep=numCuts-1;axialsweep $>=0$;axialsweep--)\{

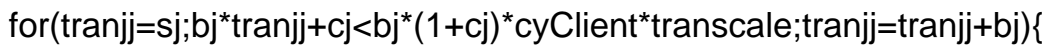

for(tranii=si;bi*tranii+ci $<\mathrm{bi}^{\star}(1+\mathrm{ci})^{\star}{ }^{*} \mathrm{cxClient}{ }^{\star}$ transcale; tranii=tranii+bi) \{

ii=tranii;

$\mathrm{jj}=$ tranjj;

yper=cyClient*transcale-jj;

yper=yper/cyClient/transcale;

y=ymin+yper ${ }^{\star}(y \max -y \min )$;

axialmin)/numCuts)/axialstretch;

axial=(axialmin+axialsweep ${ }^{\star}($ axialmax-

$/ / x=32$

st=axial*2;

TextOut(hdc,180-

60,0,szBuffer,wsprintf(szBuffer,TEXT("\%d.\%d>"),st,decz));

//xper=cxClient*transcale-ii;

//xper=xper/cxClient/transcale;

$/ / x=x \min +x_{p e r}^{*}(x \max -x \min )$;

xper $=$ cxClient ${ }^{\star}$ transcale-ii;

xper=xper/cxClient/transcale;

$x=x \min +x_{p e r}^{*}(x \max -x \min )$;

//axial $=\left(\right.$ axialmin $+x^{*}{ }^{*}($ axialmax-axialmin $\left.)\right) / 2$;

$x y z[k k][3], 2$.$) ;$

if(initializedbmap==0)\{//initializedbmap=1;

$\mathrm{z}=0$;

for $(k k=0 ; k k<n n ; k k++)\{$

$\mathrm{ri}=\operatorname{pow}(\mathrm{x}-\mathrm{xyz}[\mathrm{kk}][0], 2)+.\operatorname{pow}(y-x y z[k k][1], 2)+.\operatorname{pow}($ axial-

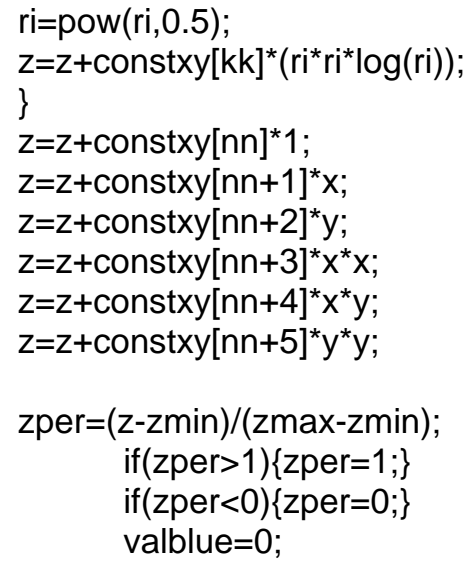




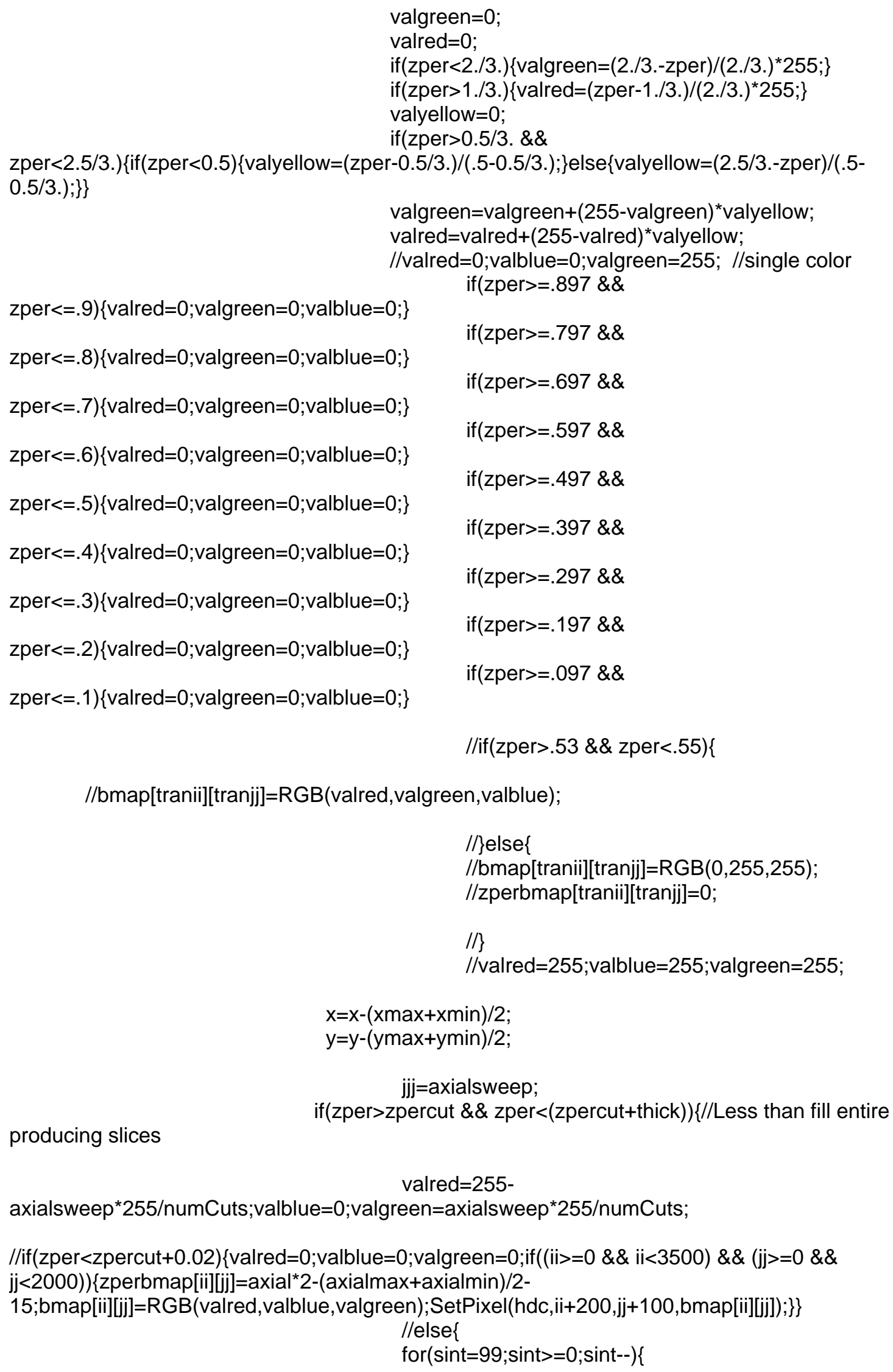


valred $=255-$

axialsweep ${ }^{\star 255 / n u m C u t s ; v a l b l u e=0 ; v a l g r e e n=a x i a l s w e e p * 255 / n u m C u t s ; ~}$

$$
\begin{aligned}
& \text { if(jjj\%3==0) }\{\text { valred }=0 ; \text { valblue }=0 ; \text { valgreen }=0 ;\} \\
& \text { //else }\{\text { valred }=255 \text {; valblue }=0 \text {; valgreen }=0 ;\} \\
& \text { //if(sint }<=10)\{\text { valred }=0 ; \text { valblue }=0 \text {; valgreen }=0 ;\} \\
& \text { //if(sint }>=90)\{\text { valred }=0 ; \text { valblue }=0 ; \text { valgreen }=0 ;\} \\
& \text { //if(sint } \% 11==0)\{\text { valred }=0 \text {; valblue }=0 ; \text { valgreen }=0 ;\} \\
& \text { //if(sint } \% 12==0)\{\text { valred }=0 ; \text { valblue }=0 ; \text { valgreen }=0 ;\} \\
& \text { //if(jjj\%3==0) }\{\text { valred }=0 ; \text { valblue }=0 ; \text { valgreen }=0 ;\}
\end{aligned}
$$

$x$ display $=x^{\star}$ transmatrix $[0][0]+y^{\star}$ transmatrix[0][1] $+\left(\sin t^{\star} 0.01^{*}(\right.$ axialmax-

axialmin)/numCuts+axial*axialstretch-(axialmax+axialmin)/2)*transmatrix[0][2]+transmatrix[0][3];

ydisplay $=x^{\star}$ transmatrix $[1][0]+y^{*}$ transmatrix[1][1]+(sint*0.01*(axialmax-

axialmin)/numCuts+axial ${ }^{\star}$ axialstretch-(axialmax+axialmin)/2)*transmatrix[1][2]+transmatrix[1][3];

$\mathrm{ii}=\mathrm{cxClient}-(\mathrm{xdisplay}-\mathrm{xmin}) /(\mathrm{xmax}-\mathrm{xmin})^{*} \mathrm{cxClient;}$

$\mathrm{jj}=$ cyClient-(ydisplay-ymin)/(ymax-ymin) ${ }^{\star}$ cyClient;

if((ii $>=0 \& \& \mathrm{ii}<3500) \& \&(\mathrm{jj}>=0 \& \& \mathrm{jj}<3500))\{$ //if((ii==0 \||

$\mathrm{ii}==3499) \|(\mathrm{jj}==0|| \mathrm{jj}==3499))\{$ valred $=0 ;$ valblue $=255$; valgreen $=0 ;\}$

zperbmap $[\mathrm{ii}][j]]=\sin { }^{\star} 0.01 *($ axialmax-

axialmin)/numCuts+axial*axialstretch-(axialmax+axialmin)/2;

bmap[ii][j]]=RGB(valred,valblue,valgreen);SetPixel(hdc,ii+100,jj+100,bmap[ii][ij]);

\}// $\}$

\}

\}

if(initializedbmap $==1)\{$

$\mathrm{x}=\mathrm{x}-(\mathrm{xmax}+\mathrm{xmin}) / 2$;

$\mathrm{y}=\mathrm{y}-(\mathrm{ymax}+\mathrm{ymin}) / 2$;

zper=axialªxialstretch-(axialmax+axialmin)/2-15;

$x$ display $=x^{\star}$ transmatrix $[0][0]+y^{\star}$ transmatrix[0][1]+zper*transmatrix[0][2]+transmatrix[0][3];

$y d i s p l a y=x * t r a n s m a t r i x[1][0]+y^{*}$ transmatrix[1][1]+zper*transmatrix[1][2]+transmatrix[1][3];

$\mathrm{ii}=\mathrm{cxClient}-(\mathrm{xdisplay}-\mathrm{xmin}) /(\mathrm{xmax}-\mathrm{xmin})^{\star} \mathrm{cxClient;}$

$\mathrm{jj}=$ cyClient-(ydisplay-ymin)/(ymax-ymin)* ${ }^{\star}$ cyClient;

SetPixel(hdc,ii+100,jj+100,bmap[ii][j]]);

\}

//if((ii >=0 \&\& ii $<3500) \& \&(j j>=0 \& \&$

$\mathrm{jj}<2000))\{$ SetPixel(hdc, $\mathrm{ii}+200, \mathrm{jj}+100$, bmap[ii][j]]); $\}$

initializedbmap=1;

ii=180; 
for $(k \mathrm{k}=0 ; \mathrm{kk}<100 ; \mathrm{kk}++)\{$

valred=255-k ${ }^{\star 2} 255 / 100$; valblue=0; valgreen $=k k^{\star} 255 / 100$;

for $\left(j \mathrm{j}=0 ; j \mathrm{j}<2{ }^{*}\right.$ ptwidth;jj++) \{

SetPixel(hdc,ii+20-ptwidth+kk,10+jj-

ptwidth,RGB(valred,valblue,valgreen));

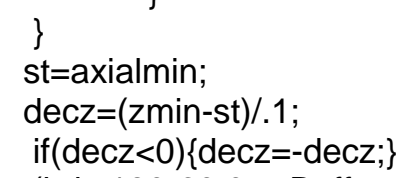

TextOut(hdc,280+45,0,szBuffer,wsprintf(szBuffer,TEXT("<\%d"),st));

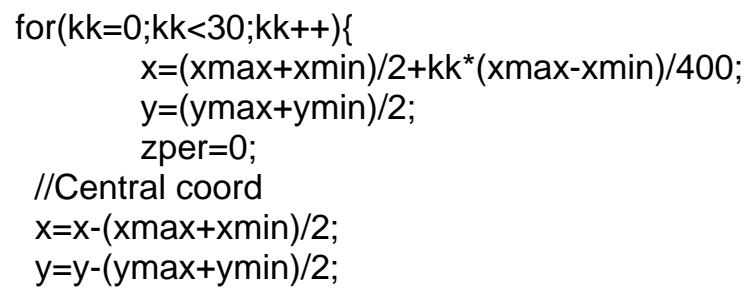

$x d i s p l a y=x^{\star}$ transmatrix $[0][0]+y^{\star}$ transmatrix[0][1]+zper*transmatrix[0][2]+transmatrix[0][3];

ydisplay $=x^{\star}$ transmatrix[1][0]+y*transmatrix[1][1]+zper*transmatrix[1][2]+transmatrix[1][3];

$$
\begin{aligned}
& x \text { display=xdisplay }-(x \max +x \min ) / 2 \\
& \text { ydisplay=ydisplay-(ymax+ymin)/2; }
\end{aligned}
$$

ii $=$ cxClient-(xdisplay-xmin)/(xmax-xmin)*cxClient;

$\mathrm{jj}=$ cyClient-(ydisplay-ymin)/(ymax-ymin) ${ }^{\star}$ cyClient;

SetPixel(hdc,ii+20,jj+20,RGB(0,0,255));

\}

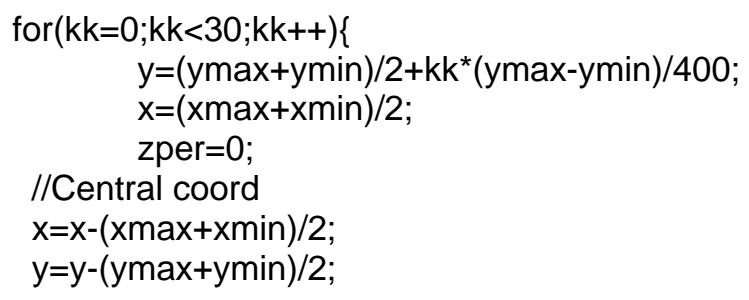

$x d i s p l a y=x^{\star}$ transmatrix $[0][0]+y^{\star}$ transmatrix[0][1]+zper*transmatrix[0][2]+transmatrix[0][3];

$y$ display $=x^{\star}$ transmatrix[1][0]+y*transmatrix[1][1]+zper*transmatrix[1][2]+transmatrix[1][3];

$x$ display=xdisplay $-(x \max +x \min ) / 2$;

ydisplay=ydisplay-(ymax+ymin)/2; 
$\mathrm{ii}=\mathrm{cxClient}-(\mathrm{xdisplay}-\mathrm{xmin}) /(\mathrm{xmax}-\mathrm{xmin})^{\star} \mathrm{cxClient;}$

$\mathrm{jj}=$ cyClient-(ydisplay-ymin) $/(\mathrm{ymax}-\mathrm{ymin})^{\star} \mathrm{cyClient;}$

SetPixel(hdc,ii+20,jj+20,RGB(255,0,255));

\}

$$
\begin{aligned}
& \text { for }(k k=0 ; k k<40 ; k k++)\{ \\
& \mathrm{x}=(\mathrm{xmax}+\mathrm{xmin}) / 2 \text {; } \\
& y=(y \max +y \min ) / 2 \text {; } \\
& \text { zper }=\mathrm{kk}^{\star} 6 / 40 \text {; }
\end{aligned}
$$

$x$ display $=x^{\star}$ transmatrix $[0][0]+y^{\star}$ transmatrix[0][1]+zper*transmatrix[0][2]+transmatrix[0][3];

ydisplay $=x^{\star}$ transmatrix[1][0]+y*transmatrix[1][1]+zper*transmatrix[1][2]+transmatrix[1][3];

xdisplay $=x$ display $-(x \max +x \min ) / 2$

ydisplay=ydisplay-(ymax+ymin)/2;

$\mathrm{ii}=\mathrm{cxClient}-(\mathrm{xdisplay}-\mathrm{xmin}) /(\mathrm{xmax}-\mathrm{xmin}){ }^{*} \mathrm{cxClient}$;

jj=cyClient-(ydisplay-ymin)/(ymax-ymin)* cyClient;

SetPixel(hdc,ii+20,jj+20,RGB(0,0,0));

//End

return 0 ;

case WM_USER + 756:

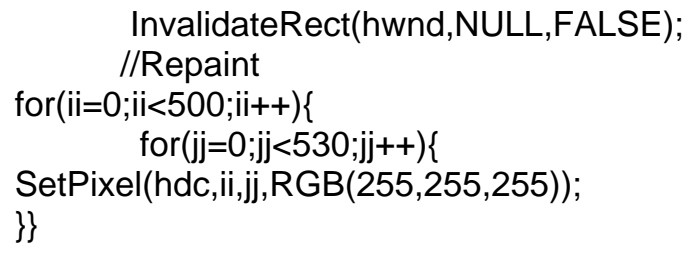

return 0 ;

case WM_DESTROY:

PostQuitMessage (0) ;

\}

return 0 ;

\}

return DefWindowProc (hwnd, message, wParam, IParam) ; 